\title{
Radical Pentafluoroethylation of Unactivated Alkenes Using $\mathrm{CuCF}_{2} \mathrm{CF}_{3}$
}

Xinkan Yang and Gavin Chit Tsui*

Department of Chemistry, The Chinese University of Hong Kong, Shatin, New Territories, Hong Kong SAR.

Table of Contents

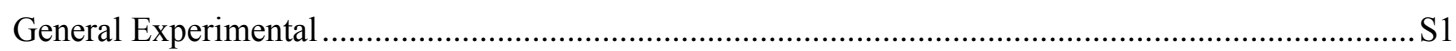

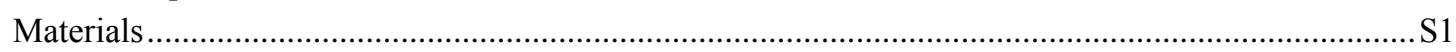

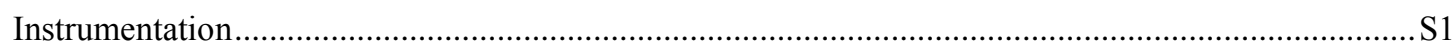

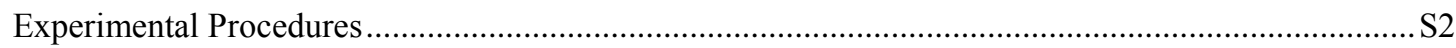

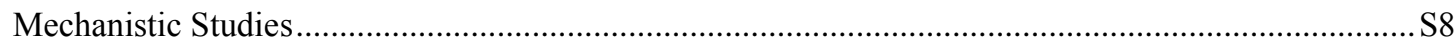

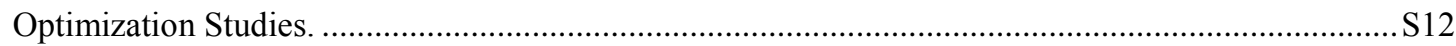

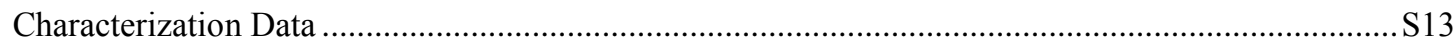

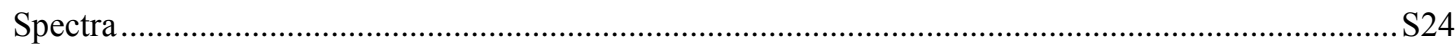

General Experimental. Analytical thin layer chromatography (TLC) was performed with EM Science silica gel 60 F254 aluminum plates. Visualization was done under a UV lamp (254 nm) and by immersion in ethanolic phosphomolybdic acid (PMA) or potassium permanganate $\left(\mathrm{KMnO}_{4}\right)$, followed by heating using a heat gun. Organic solutions were concentrated by rotary evaporation at $23-35{ }^{\circ} \mathrm{C}$. Purification of reaction products were generally done by flash column chromatography with Grace Materials Technologies 230-400 mesh silica gel.

Materials. Halocarbon 125-Pentafluoroethane (Purity: $99.0 \% \mathrm{~min} ., 9.1 \mathrm{~kg}$ in $16 \mathrm{~L}$ size cylinder) was purchased from SCIENTIFIC GAS ENGINEERING CO., LTD. Copper(I) chloride (extra pure, 99.99\%) was purchased from Acros. Potassium tert-butoxide (97\%) was purchased from Alfa Aesar. Anhydrous DMF and TREAT.HF was purchased from J\&K Scientific. Other chemicals for substrates preparation were purchased from Acros, J\&K Scientific, Aldrich and Dikemann.

Instrumentation. Proton nuclear magnetic resonance spectra $\left({ }^{1} \mathrm{H}\right.$ NMR) spectra, carbon nuclear magnetic resonance spectra $\left({ }^{13} \mathrm{C}\right.$ NMR $)$ and fluorine nuclear magnetic resonance spectra $\left({ }^{19} \mathrm{~F} \mathrm{NMR}\right)$ were recorded at $23{ }^{\circ} \mathrm{C}$ on a Bruker 400 spectrometer in $\mathrm{CDCl}_{3}\left(400 \mathrm{MHz}\right.$ for ${ }^{1} \mathrm{H}, 101 \mathrm{MHz}$ for ${ }^{13} \mathrm{C}$ and 376 $\mathrm{MHz}$ for ${ }^{19} \mathrm{~F}$ ) and Bruker 500 spectrometer in $\mathrm{CDCl}_{3}\left(500 \mathrm{MHz}\right.$ for ${ }^{1} \mathrm{H}, 126 \mathrm{MHz}$ for ${ }^{13} \mathrm{C}$ and $470 \mathrm{MHz}$ for ${ }^{19} \mathrm{~F}$ ). Chemical shifts for protons were reported as parts per million in $\delta$ scale using solvent residual peak $\left(\mathrm{CHCl}_{3}\right.$ : $\left.7.26 \mathrm{ppm}\right)$ or tetramethylsilane $(0.00 \mathrm{ppm})$ as internal standards. Chemical shifts of ${ }^{13} \mathrm{C}$ NMR spectra were reported in ppm from the central peak of $\mathrm{CDCl}_{3}(77.16 \mathrm{ppm})$ on the $\delta$ scale. Chemical shifts of ${ }^{19} \mathrm{~F}$ NMR are reported as parts per million in $\delta$ scale using benzotrifluoride $(-63.72 \mathrm{ppm})$ as internal standards. Data are represented as follows: chemical shift, integration, multiplicity $(s=$ singlet, $\mathrm{d}=$ doublet, $\mathrm{t}=$ triplet, $\mathrm{q}=$ quartet, $\mathrm{qn}=$ quintuplet, $\mathrm{sx}=$ sextet, $\mathrm{sp}=$ septuplet, $\mathrm{m}=$ multiplet, $\mathrm{br}=$ broad), and coupling constant $(J, \mathrm{~Hz})$. High resolution mass spectra (HRMS) were obtained on a Bruker SolariX 9.4T ICR Mass Spectrometer or Thermo Q Exactive Focus Orbitrap Mass Spectrometer. The melting points were obtained on a Stuart ${ }^{\mathrm{TM}}$ SMP40 automatic melting point apparatus. The control experiment results were obtained on a Shimadzu GCMS-QP2010 SE GC MS Spectrometer. 


\section{Experimental Procedures:}

Modified procedure for the preparation of pentafluoroethane-derived $\left[\mathrm{CuCF}_{2} \mathrm{CF}_{3}\right]$ reagent: $^{1}$

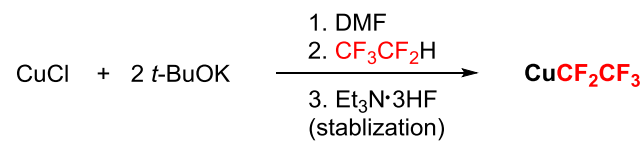

In a glove box, to a glass tube was charged $\mathrm{CuCl}(200 \mathrm{mg}, 2.0 \mathrm{mmol}), t$-BuOK (472 mg, $4.0 \mathrm{mmol})$ and a stirrer bar. The flask was sealed with a septum, brought out of the glove box and put under an argon atmosphere. Degassed DMF (1.0 mL) was added via syringe and the mixture was vigorously stirred at room temperature for $30 \mathrm{~min}$. Then pentafluoroethane $\left(\mathrm{CF}_{3} \mathrm{CF}_{2} \mathrm{H}\right)$ was bubbled into the mixture by using a needle connected to the $\mathrm{CF}_{3} \mathrm{CF}_{2} \mathrm{H}$ cylinder at room temperature for $2.5 \mathrm{~min}$. After removing the $\mathrm{CF}_{3} \mathrm{CF}_{2} \mathrm{H}$ inlet, the mixture was stirred for $5 \mathrm{~min}$ and $\mathrm{Et}_{3} \mathrm{~N} \cdot 3 \mathrm{HF}(326 \mu \mathrm{L}, 2.0 \mathrm{mmol})$ was slowly added under argon and the mixture was stirred for another $5 \mathrm{~min}$. A slightly greyish yellow solution with white precipitates was obtained as the $\left[\mathrm{CuCF}_{2} \mathrm{CF}_{3}\right]$ solution in $\mathrm{DMF}(\sim 87 \%, \sim 0.90 \mathrm{M})$.

${ }^{19} \mathrm{~F}$ NMR of freshly prepared $\mathrm{CuCF}_{2} \mathrm{CF}_{3}$ reagent (in DMF, under argon, internal standard = $\left.\mathrm{PhCF}_{3}\right)$ :
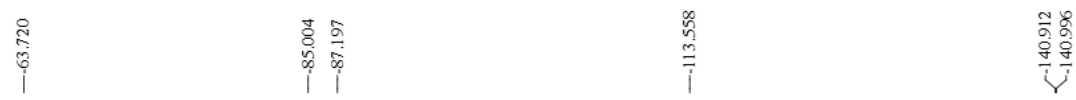

$\mathrm{CuCF}_{2} \mathrm{CF}_{3}$
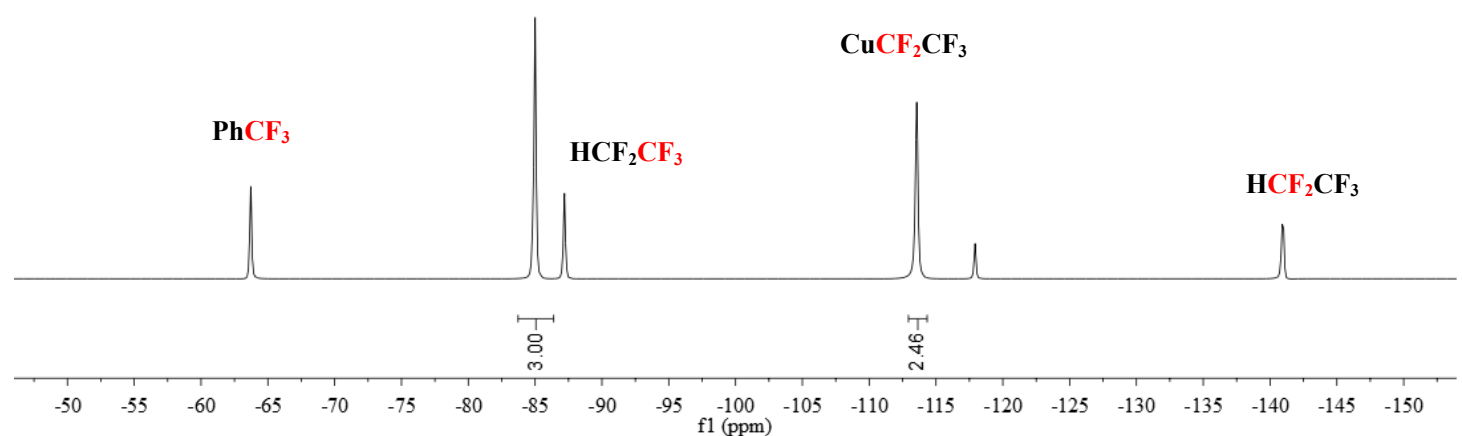

1 Lishchynskyi, A.; Grushin, V. V. J. Am. Chem. Soc. 2013, 135, 12584. 
${ }^{19}$ F NMR of the $\mathrm{CuCF}_{2} \mathrm{CF}_{3}$ reagent (0.1 M in DMF) over time when open to air:

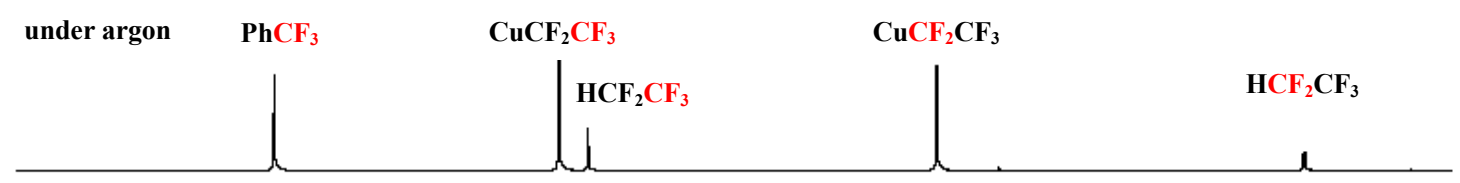

open to air 20 mins

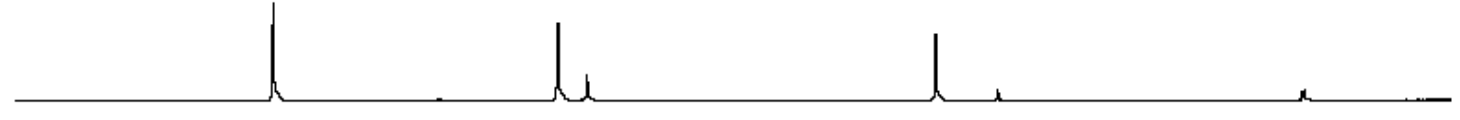

open to air $1 \mathrm{~h}$

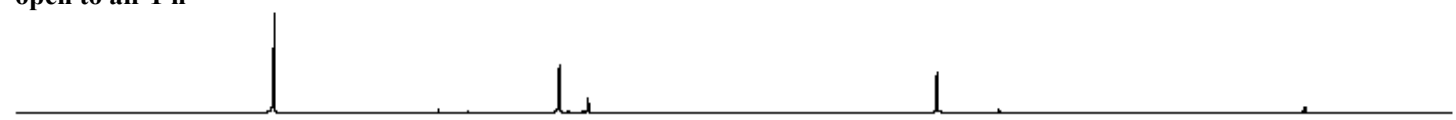

open to air $3 \mathrm{~h}$

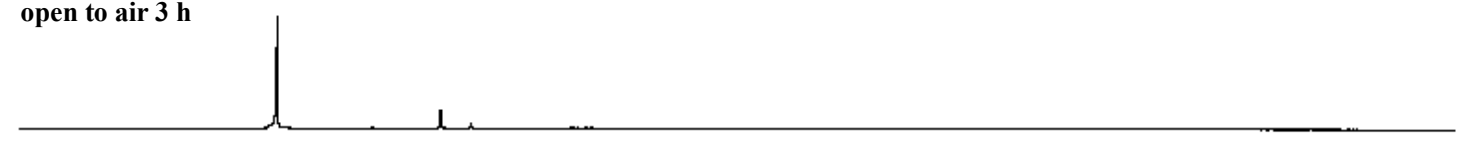

open to air $6 \mathrm{~h}$

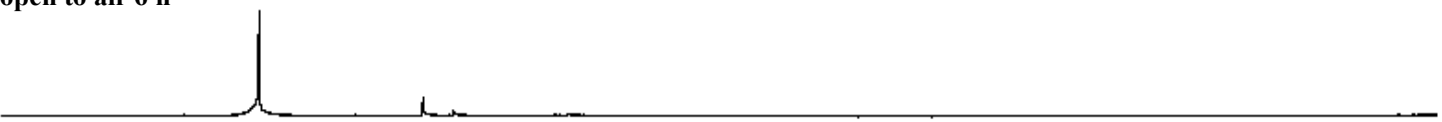

open to air $24 \mathrm{~h}$

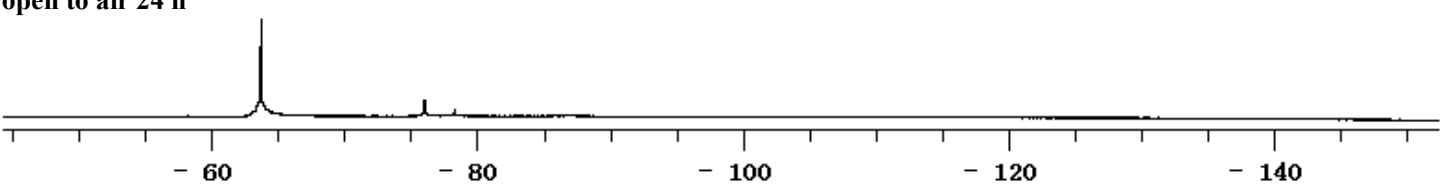

General procedure (cf. Scheme 2):

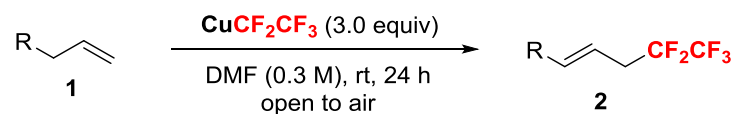

Under air, to a glass tube equipped with a magnetic stir bar and alkene $\mathbf{1}(0.3 \mathrm{mmol})$ was added above freshly prepared $\left[\mathrm{CuCF}_{2} \mathrm{CF}_{3}\right](1.0 \mathrm{~mL}, 0.90 \mathrm{mmol}$ in $\mathrm{DMF})$ at $0{ }^{\circ} \mathrm{C}$. Then the tube was warmed to room temperature and stirred for $24 \mathrm{~h}$. The color slowly changed from greyish yellow to dark red. The $\mathrm{E} / \mathrm{Z}$ ratio was determined by ${ }^{19} \mathrm{~F}$ NMR of the crude mixture. The reaction mixture was quenched with aq. sat. sodium potassium tartrate, extracted with diethyl ether three times. The organic layers were combined, washed with water then brine, dried over anhydrous $\mathrm{Na}_{2} \mathrm{SO}_{4}$, filtered and concentrated by rotary evaporator. The crude product was purified by flash column chromatography on silica gel to afford the desired product 2. 
Substrates 1:

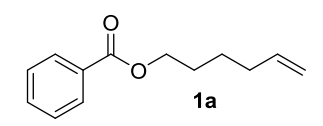<smiles>C=CCCCCOC(=O)c1ccc(OC)cc1</smiles><smiles>C=CCCCCOC(=O)c1ccc(F)cc1</smiles><smiles>C=CCCCCOC(=O)c1ccc(Cl)cc1</smiles><smiles>C=CCCCCOC(=O)c1ccc(Br)cc1</smiles><smiles>C=CCCCCOC(=O)c1ccc(I)cc1</smiles><smiles>C=CCCCCOC(=O)c1ccco1</smiles><smiles>C=CCCCCOC(=O)c1cccs1</smiles><smiles>C=CCCCOC(=O)c1ccccn1</smiles><smiles>C=CCCCCOc1ccc(I)cc1</smiles><smiles>C=CCCCOc1ccc(C(C)=O)cc1</smiles><smiles>C=CCCCCOc1ccc(C=O)cc1</smiles><smiles>C=CCCCCOCc1ccccc1</smiles>

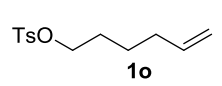<smiles>C=CCCCCOC(=O)CCCCCBr</smiles><smiles>C=CCCCCCCCCC(=O)OC</smiles><smiles>C=CCCCCCCCCC(=O)N1CCOCC1</smiles><smiles>C=CCCCCCCCC1CO1</smiles><smiles>C=CCCCCCCCCCO</smiles><smiles>C=CCCCCCCCCC(=O)O</smiles><smiles>C=CCCCCOc1ccc2c(c1)CCC1C3CCC4(C)C(=O)CCC3C4CCC21C</smiles><smiles>C1=CCCCC1</smiles><smiles>C=CCCCCOc1ccc2c(C)cc(=O)oc2c1</smiles><smiles>C=CCCCCOc1c(-c2ccccc2)oc2ccccc2c1=O</smiles><smiles>C=CC(=O)Oc1ccc2ccccc2c1</smiles><smiles>C=C1CCC(c2ccccc2)CC1</smiles><smiles>C=CCC(C)(CC=C)C(=O)OCC</smiles>
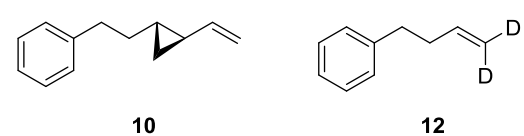

Alkenes 1a-1p, 1r, 1w-1ab, 10, 12 were synthesized according to literature procedure. ${ }^{2} \mathbf{1 q}, \mathbf{1 s - 1 v}$, 1ac-1ad, 8 were commercially available.

2 (a) Yang, X.; Tsui, G. C. Org. Lett. 2019, 21, 1521. (b) Ye, K.-Y.; McCallum, T.; Lin, S. J. Am. Chem. Soc. 2019, 141, 9548-9554. (c) Rigby, C. L.; Dixon, D. J. Chem. Commun. 2008, 32, 3798. 
1 mmol scale reaction ( $c f$. Scheme 2, compound $2 \mathrm{~b})$ :

\section{Large scale (14 mmol) preparation of pentafluoroethane-derived $\left[\mathrm{CuCF}_{2} \mathrm{CF}_{3}\right]$ reagent:}

In a glove box, to a $25 \mathrm{~mL}$ round-bottom flask was charged $\mathrm{CuCl}(1.40 \mathrm{~g}, 14 \mathrm{mmol}), t$-BuOK (3.30 g, $28.0 \mathrm{mmol}$ ) and a stirrer bar. The flask was sealed with a septum, brought out of the glove box and put under an argon atmosphere. Degassed DMF $(7.0 \mathrm{~mL})$ was added via syringe with a water bath, then the water bath was removed and the mixture was vigorously stirred at room temperature for $45 \mathrm{~min}$. Pentafluoroethane $\left(\mathrm{CF}_{3} \mathrm{CF}_{2} \mathrm{H}\right)$ was bubbled into the mixture by using a needle connected to the $\mathrm{CF}_{3} \mathrm{CF}_{2} \mathrm{H}$ cylinder at room temperature for $5 \mathrm{~min}$. After removing the $\mathrm{CF}_{3} \mathrm{CF}_{2} \mathrm{H}$ inlet, the mixture was stirred for 5 min and $\mathrm{Et}_{3} \mathrm{~N} \cdot 3 \mathrm{HF}(2.30 \mathrm{~mL}, 14 \mathrm{mmol})$ was slowly added under argon and the mixture was stirred for another $5 \mathrm{~min}$. A slightly greyish yellow solution with white precipitates was obtained as the $\left[\mathrm{CuCF}_{2} \mathrm{CF}_{3}\right]$ solution in DMF $(\sim 0.90 \mathrm{M})$.

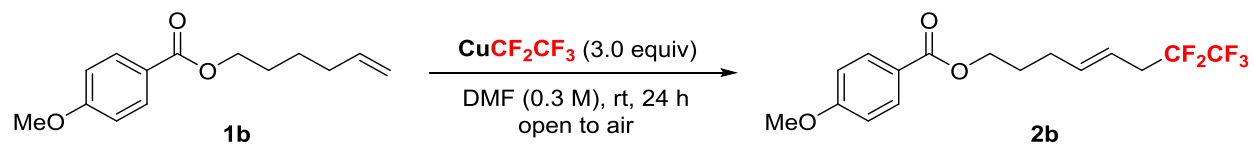

Under air, to a $50 \mathrm{~mL}$ glass tube equipped with a magnetic stir bar and alkene $\mathbf{1 b}$ (234 $\mathrm{mg}, 1.0 \mathrm{mmol})$ was added above freshly prepared $\left[\mathrm{CuCF}_{2} \mathrm{CF}_{3}\right](3.30 \mathrm{~mL}, 3.0 \mathrm{mmol}$ in $\mathrm{DMF})$ at $0{ }^{\circ} \mathrm{C}$. Then the tube was warmed to room temperature and stirred for $24 \mathrm{~h}$. The color slowly changed from greyish yellow to dark red. The $\mathrm{E} / \mathrm{Z}$ ratio was determined by ${ }^{19} \mathrm{~F}$ NMR of the crude mixture. The reaction mixture was quenched with aq. sat. sodium potassium tartrate, extracted with diethyl ether three times. The organic layers were combined, washed with water then brine, dried over anhydrous $\mathrm{Na}_{2} \mathrm{SO}_{4}$, filtered and concentrated by rotary evaporator. The crude product was purified by flash column chromatography on silica gel to afford the desired product $\mathbf{2 b}$ as a colorless oil $(289 \mathrm{mg}, 0.82 \mathrm{mmol}, 82 \%, \mathrm{E} / \mathrm{Z}=95: 5$, allylic $:$ vinylic $=27: 1)$. $\mathrm{R}_{\mathrm{f}}=0.40$ (Hexane $\left.: \mathrm{Et}_{2} \mathrm{O}=5: 1\right)$. 
Further transformations ( $c f$. Scheme 3):<smiles>COc1ccc(C(=O)OCCCC=CCC(F)(F)C(F)(F)C(F)(F)C(F)(F)C(F)(F)C(F)(F)C(F)(F)C(F)(F)F)cc1</smiles>

To a $10 \mathrm{~mL}$ round-bottom flask equipped with a magnetic stir bar and $\mathbf{2 b}(70.4 \mathrm{mg}, 0.2 \mathrm{mmol})$ was added $\mathrm{MeOH}(4.0 \mathrm{~mL})$ and $\mathrm{Pd} / \mathrm{C}(21.1 \mathrm{mg}, 0.02 \mathrm{mmol})$, the mixture was evacuated and refilled with $\mathrm{H}_{2}$ for three times. Then stirred at room temperature for $12 \mathrm{~h}$ under a $\mathrm{H}_{2}$ balloon. After completion (monitored by TLC), filtered and washed with dichloromethane for three times, the combined filtrate was concentrated by rotary evaporator. The crude residue was directly purified by flash column chromatography on silica gel to afford the desired product $3(0.18 \mathrm{mmol}, 63.7 \mathrm{mg}, 90 \%)$ as a colorless oil. $\mathrm{R}_{\mathrm{f}}=0.5$ (hexane : $\left.\mathrm{Et}_{2} \mathrm{O}=5: 1\right) .{ }^{1} \mathbf{H}$ NMR $\left(400 \mathrm{MHz}, \mathrm{CDCl}_{3}\right): \delta 7.99(\mathrm{~d}, J=8.8 \mathrm{~Hz}, 2 \mathrm{H}), 6.91(\mathrm{~d}, J$ $=8.8 \mathrm{~Hz}, 2 \mathrm{H}), 4.28(\mathrm{t}, J=6.6 \mathrm{~Hz}, 2 \mathrm{H}), 3.84(\mathrm{~s}, 3 \mathrm{H}), 2.08-1.94(\mathrm{~m}, 2 \mathrm{H}), 1.77(\mathrm{~m}, 2 \mathrm{H}), 1.61(\mathrm{~m}, 2 \mathrm{H}), 1.51-$ $1.41(\mathrm{~m}, 4 \mathrm{H}) \mathrm{ppm} .{ }^{13} \mathbf{C}$ NMR $\left(101 \mathrm{MHz}, \mathrm{CDCl}_{3}\right): \delta 166.5,163.4,131.6,123.2,119.3\left(\mathrm{qt}, J_{C-F}=286.3\right.$ $\left.\mathrm{Hz}, J_{C-F}=36.6 \mathrm{~Hz}\right), 115.9\left(\mathrm{tq}, J_{C-F}=252.3 \mathrm{~Hz}, J_{C-F}=37.6 \mathrm{~Hz}\right), 113.7,64.6,55.4,30.7\left(\mathrm{t}, J_{C-F}=22.2 \mathrm{~Hz}\right)$, 28.8, 28.6, 25.8, $20.3\left(\mathrm{t}, J_{C-F}=3.4 \mathrm{~Hz}\right)$ ppm. ${ }^{19}$ F NMR $\left(376 \mathrm{MHz}, \mathrm{CDCl}_{3}\right): \delta-86.43(\mathrm{~s}, 3 \mathrm{~F}),-119.21(\mathrm{t}, J$ $=18.4 \mathrm{~Hz}, 2 \mathrm{~F})$. HRMS m/z (ESI): calcd. for $\mathrm{C}_{16} \mathrm{H}_{19} \mathrm{~F}_{5} \mathrm{O}_{3} \mathrm{Na}[\mathrm{M}+\mathrm{Na}]^{+}: 377.1147$; found: 377.1144 .<smiles>COc1ccc(C(=O)OCCC/C=C/CC(F)(F)F)cc1</smiles>

Followed a literature procedure ${ }^{3}$ : a solution of $m$-CPBA $(119 \mathrm{mg}, 0.48 \mathrm{mmol})$ in $\mathrm{CHCl}_{3}(1.6$ $\mathrm{mL})$ was added to a solution of $\mathbf{2} \mathbf{b}(70.4 \mathrm{mg}, 0.2 \mathrm{mmol})$ in $\mathrm{CHCl}_{3}(0.6 \mathrm{~mL})$ at $0{ }^{\circ} \mathrm{C}$ and then warmed to room temperature. After the solution was stirred for $12 \mathrm{~h}, \mathrm{CH}_{2} \mathrm{Cl}_{2}$ was added, the organic phase was washed with sat. aq. $\mathrm{Na}_{2} \mathrm{~S}_{2} \mathrm{O}_{3}$ and $\mathrm{NaHCO}_{3}$, then brine, dried over anhydrous $\mathrm{Na}_{2} \mathrm{SO}_{4}$, filtered and concentrated by rotary evaporator. The residue was purified by flash column chromatography on silica gel (hexane $\left./ \mathrm{Et}_{2} \mathrm{O}\right)$ and obtained product $4(0.14 \mathrm{mmol}, 52.2 \mathrm{mg}, 71 \%, \mathrm{dr}=93: 7)$ as a yellow solid, melting point: $38.0-38.7^{\circ} \mathrm{C}, \mathrm{R}_{\mathrm{f}}=0.2$ (hexane : $\mathrm{Et}_{2} \mathrm{O}=5$ : 1). Major isomer: ${ }^{1} \mathbf{H} \mathbf{N M R}\left(400 \mathrm{MHz}, \mathrm{CDCl}_{3}\right)$ : $\delta 7.97(\mathrm{~d}, J=8.8 \mathrm{~Hz}, 2 \mathrm{H}), 6.90(\mathrm{~d}, J=8.8 \mathrm{~Hz}, 2 \mathrm{H}), 4.32(\mathrm{td}, J=6.4 \mathrm{~Hz}, J=2.0 \mathrm{~Hz}, 2 \mathrm{H}), 3.83(\mathrm{~s}, 3 \mathrm{H})$, $2.98(\mathrm{t}, J=5.6 \mathrm{~Hz}, 1 \mathrm{H}), 2.84(\mathrm{t}, J=5.6 \mathrm{~Hz}, 1 \mathrm{H}), 2.44-2.29(\mathrm{~m}, 1 \mathrm{H}), 2.25-2.11(\mathrm{~m}, 1 \mathrm{H}), 1.96-1.85(\mathrm{~m}$, 2H), 1.82-1.74 (m, 1H), 1.71-1.64 (m, 1H) ppm. ${ }^{13} \mathbf{C}$ NMR (101 MHz, $\left.\mathrm{CDCl}_{3}\right): \delta 166.3,163.5,131.6$, $122.7,119.2\left(\mathrm{qt}, J_{C-F}=286.2 \mathrm{~Hz}, J_{C-F}=35.8 \mathrm{~Hz}\right), 114.9\left(\mathrm{tq}, J_{C-F}=253.7 \mathrm{~Hz}, J_{C-F}=38.7 \mathrm{~Hz}\right), 113.7,63.9$, $57.4,55.4,50.7\left(\mathrm{t}, J_{C-F}=5.2 \mathrm{~Hz}\right), 34.6\left(\mathrm{t}, J_{C-F}=21.7 \mathrm{~Hz}\right), 28.4,25.2$ ppm. ${ }^{19} \mathbf{F} \mathbf{N M R}\left(376 \mathrm{MHz}, \mathrm{CDCl}_{3}\right)$ : $\delta-86.37$ (s, 3F), -117.15 (m, 2F). HRMS m/z (ESI): calcd. for $\mathrm{C}_{16} \mathrm{H}_{17} \mathrm{~F}_{5} \mathrm{O}_{4} \mathrm{Na}[\mathrm{M}+\mathrm{Na}]^{+}:$391.0939; found: 391.0937.

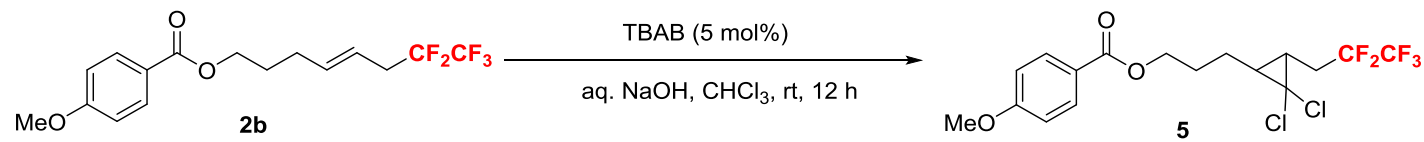

Followed a literature procedure ${ }^{3}$ : a glass tube charge with a stirred bar was added $\mathbf{2 b}$ (70.4 mg,

3 Xu, J.; Fu, Y.; Luo, D.-F.; Jiang, Y.-Y.; Xiao, B.; Liu, Z.-J.; Gong, T.-J.; Liu, L. J. Am. Chem. Soc. 2011, 133, 15300 . 
$0.2 \mathrm{mmol}), \mathrm{TBAB}(3.2 \mathrm{mg}, 0.01 \mathrm{mmol})$ and $17 \mathrm{M}$ aq. $\mathrm{NaOH}(1.2 \mathrm{~mL}, 20.0 \mathrm{mmol})$, then $\mathrm{CHCl}_{3}(0.3$ $\mathrm{mL} / 0.3 \mathrm{~mL}$ ) was added in two portions. The mixture was stirred at room temperature until $\mathbf{2} \mathbf{b}$ was fully consumed (monitored by GC MS). Diluted with $\mathrm{H}_{2} \mathrm{O}$ and $\mathrm{CH}_{2} \mathrm{Cl}_{2}$, extracted with $\mathrm{CH}_{2} \mathrm{Cl}_{2}$ for three times. The combined organic phase was washed with $\mathrm{H}_{2} \mathrm{O}$ and brine, dried over anhydrous $\mathrm{Na}_{2} \mathrm{SO}_{4}$, filtered and concentrated by rotary evaporator. The residue was purified by flash column chromatography on silica gel (hexane/ $\left.\mathrm{Et}_{2} \mathrm{O}\right)$ and obtained product $5(0.18 \mathrm{mmol}, 78.1 \mathrm{mg}, 90 \%, \mathrm{dr}=93$ : 7) as a yellowish liquid, $\mathrm{R}_{\mathrm{f}}=0.5$ (hexane : $\mathrm{Et}_{2} \mathrm{O}=5: 1$ ). Major isomer: ${ }^{1} \mathbf{H} \mathbf{N M R}\left(400 \mathrm{MHz}, \mathrm{CDCl}_{3}\right): \delta$ $7.98(\mathrm{~d}, J=8.8 \mathrm{~Hz}, 2 \mathrm{H}), 6.91(\mathrm{~d}, J=8.8 \mathrm{~Hz}, 2 \mathrm{H}), 4.34(\mathrm{t}, J=6.4 \mathrm{~Hz} 2 \mathrm{H}), 3.84$ (s, 3H), 2.64-2.50 (m, 1H), 2.16-2.02 (m, 1H), 2.00-1.91 (m, 2H), 1.75 (q, $J=7.3 \mathrm{~Hz}, 2 \mathrm{H}), 1.47-1.37$ (m, 2H) ppm. ${ }^{13} \mathbf{C}$ NMR $\left(101 \mathrm{MHz}, \mathrm{CDCl}_{3}\right): \delta 166.4,163.5,131.7,122.7,119.0\left(\mathrm{qt}, J_{C-F}=286.5 \mathrm{~Hz}, J_{C-F}=35.9 \mathrm{~Hz}\right), 115.2(\mathrm{tq}$, $\left.J_{C-F}=252.9 \mathrm{~Hz}, J_{C-F}=38.1 \mathrm{~Hz}\right), 113.7,64.2,63.9,55.5,35.3,31.6\left(\mathrm{t}, J_{C-F}=21.9 \mathrm{~Hz}\right), 28.1\left(\mathrm{t}, J_{C-F}=4.0\right.$ $\mathrm{Hz}), 27.5,26.8$ ppm. ${ }^{19} \mathbf{F}$ NMR (376 MHz, $\left.\mathrm{CDCl}_{3}\right): \delta-86.23$ (s, 3F), $-118.10(\mathrm{~m}, 2 \mathrm{~F}) . \mathbf{H R M S ~ m} / \mathrm{z}(\mathrm{ESI})$ : calcd. for $\mathrm{C}_{17} \mathrm{H}_{17} \mathrm{Cl}_{2} \mathrm{~F}_{5} \mathrm{O}_{3}[\mathrm{M}+\mathrm{Na}]^{+}$: 457.0367 ; found: 457.0368 .

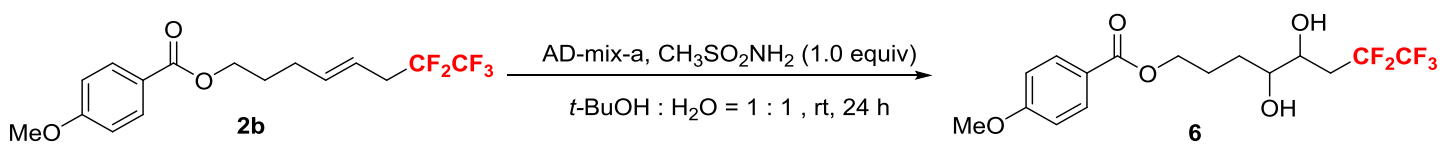

Followed a literature procedure ${ }^{3}$ : a glass tube equipped with a stirred bar was charge with $t$ $\mathrm{BuOH}(1.0 \mathrm{~mL}), \mathrm{H}_{2} \mathrm{O}(1.0 \mathrm{~mL}), \mathrm{AD}-\mathrm{mix}-\alpha(280 \mathrm{mg})$ and $\mathrm{CH}_{3} \mathrm{SO}_{2} \mathrm{Na}(19.0 \mathrm{mg}, 0.2 \mathrm{mmol})$. The mixture was stirred until both phases were clear. Then $\mathbf{2 b}(70.4 \mathrm{mg}, 0.2 \mathrm{mmol})$ was added at once and the heterogenous slurry was stirred until the $\mathbf{2} \mathbf{b}$ was fully consumed. The reaction was quenched at $0{ }^{\circ} \mathrm{C}$ by adding $\mathrm{Na}_{2} \mathrm{SO}_{3}(3.0 \mathrm{~g})$, then warm to room temperature and stirred for $1 \mathrm{~h}$, extracted with EtOAc for three times. The organic layers were combined, washed with brine, dried over anhydrous $\mathrm{Na}_{2} \mathrm{SO}_{4}$, filtered and concentrated by rotary evaporator. The crude residue was purified by flash column chromatography on silica gel (hexane/acetone) and obtained product $6(0.18 \mathrm{mmol}, 71.0 \mathrm{mg}$, $92 \%, \mathrm{dr}>20: 1$ ) as a white solid, melting point: $82.6-83.4{ }^{\circ} \mathrm{C}, \mathrm{R}_{\mathrm{f}}=0.4$ (hexane : acetone $=5: 1$ ). Major isomer: ${ }^{1} \mathbf{H}$ NMR $\left(400 \mathrm{MHz}, \mathrm{CDCl}_{3}\right): \delta 7.94(\mathrm{~d}, J=8.8 \mathrm{~Hz}, 2 \mathrm{H}), 6.88(\mathrm{~d}, J=8.8 \mathrm{~Hz}, 2 \mathrm{H}), 4.30(\mathrm{t}, J=$ $6.4 \mathrm{~Hz}, 2 \mathrm{H}), 3.99$ (s, 1H), $3.82(\mathrm{~s}, 3 \mathrm{H}), 3.54(\mathrm{~s}, 1 \mathrm{H}), 3.33(\mathrm{~d}, J=17.2 \mathrm{~Hz}, 1 \mathrm{H}), 3.13(\mathrm{~d}, J=12.8 \mathrm{~Hz}, 1 \mathrm{H})$, 2.49-2.19 (m, 2H), 1.98-1.89 (m,1H), 1.88-1.78 (m, 1H), $1.66(\mathrm{q}, J=7.2 \mathrm{~Hz}, 2 \mathrm{H}) \mathrm{ppm} .{ }^{13} \mathbf{C}$ NMR (101 $\left.\mathrm{MHz}, \mathrm{CDCl}_{3}\right): \delta 167.0,163.6,131.7,122.4,119.0\left(\mathrm{qt}, J_{C-F}=286.4 \mathrm{~Hz}, J_{C-F}=36.2 \mathrm{~Hz}\right), 115.8\left(\mathrm{tq}, J_{C-F}=\right.$ $\left.254.0 \mathrm{~Hz}, J_{C-F}=38.1 \mathrm{~Hz}\right), 113.7,73.8,67.6,64.6,55.5,35.0\left(\mathrm{t}, J_{C-F}=20.6 \mathrm{~Hz}\right), 29.9,25.2 \mathrm{ppm} .{ }^{19} \mathbf{F}$ NMR (376 MHz, $\left.\mathrm{CDCl}_{3}\right): \delta-86.83$ (s, 3F), -117.77 (m, 2F). HRMS m/z (ESI): calcd. for $\mathrm{C}_{16} \mathrm{H}_{19} \mathrm{~F}_{5} \mathrm{O}_{5}$ $[\mathrm{M}+\mathrm{Na}]^{+}:$409.1045; found: 409.1042 .

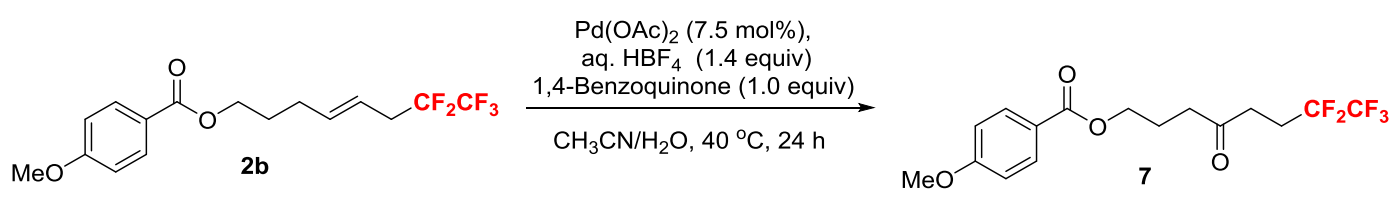

Followed a literature procedure ${ }^{4}:$ to a glass tube charges with a stirred bar was added $\mathrm{Pd}(\mathrm{OAc})_{2}$ (3.4 mg, $0.015 \mathrm{mmol}), 1,4$-benzoquinone (21.6 mg, $0.2 \mathrm{mmol})$. A mixture of $\mathrm{MeCN}(0.88 \mathrm{~mL})$ and water $(0.10 \mathrm{~mL})$ was added followed by addition of $32 \%$ aq. $\mathrm{HBF}_{4}$ solution $(60 \mu \mathrm{L})$. Then $\mathbf{2 b}(70.4$ $\mathrm{mg}, 0.2 \mathrm{mmol}$ ) was added at once and the homogeneous dark red solution was stirred for $24 \mathrm{~h}$ at 40

4 Lerch, M. M.; Morandi, B.; Wickens, Z. K.; Grubbs, R. H. Angew. Chem., Int. Ed. 2014, 53, 8654. 
${ }^{\circ} \mathrm{C}$. Diluted with sat. aq. $\mathrm{NaCl}$ solution, extracted with $\mathrm{CH}_{2} \mathrm{Cl}_{2}$. The combined organic phases were dried over anhydrous $\mathrm{Na}_{2} \mathrm{SO}_{4}$, filtered and concentrated by rotary evaporator. The crude product was then further purified by flash column chromatography on silica gel (hexane/EtOAc) to furnish the desired pure product $7(0.10 \mathrm{mmol}, 38.3 \mathrm{mg}, 52 \%, \mathrm{rr}>20: 1)$ as a white solid, melting point: $64.5-$ $65.2{ }^{\circ} \mathrm{C}, \mathrm{R}_{\mathrm{f}}=0.3$ (hexane : EtOAc $\left.=5: 1\right) .{ }^{1} \mathbf{H}$ NMR $\left(400 \mathrm{MHz}, \mathrm{CDCl}_{3}\right): \delta 7.95(\mathrm{~d}, J=8.8 \mathrm{~Hz}, 2 \mathrm{H}), 6.90$ (d, $J=8.8 \mathrm{~Hz}, 2 \mathrm{H}), 4.30$ (t, $J=6.2 \mathrm{~Hz}, 2 \mathrm{H}), 3.84(\mathrm{~s}, 3 \mathrm{H}), 2.72(\mathrm{t}, J=7.8 \mathrm{~Hz}, 2 \mathrm{H}), 2.62(\mathrm{t}, J=7.2 \mathrm{~Hz}$, 2H), 2.41-2.28 (m, 2H), 2.10-2.03 (m, 2H) ppm. ${ }^{13} \mathbf{C}$ NMR (101 MHz, $\left.\mathrm{CDCl}_{3}\right): \delta 206.1,166.4,163.6$, $131.7,122.5,119.1$ (qt, $\left.J_{C-F}=286.2 \mathrm{~Hz}, J_{C-F}=36.3 \mathrm{~Hz}\right), 115.6\left(\mathrm{tq}, J_{C-F}=252.6 \mathrm{~Hz}, J_{C-F}=38.0 \mathrm{~Hz}\right.$ ), 113.7, 63.7, 55.5, 39.2, 33.6, $24.8\left(\mathrm{t}, J_{C-F}=22.1 \mathrm{~Hz}\right), 23.0 \mathrm{ppm} .{ }^{19} \mathbf{F}$ NMR $\left(376 \mathrm{MHz}, \mathrm{CDCl}_{3}\right): \delta-86.49$ (s, 3F), -119.30 (t, $J=18.4 \mathrm{~Hz}, 2 \mathrm{~F})$. HRMS m/z (ESI): calcd. for $\mathrm{C}_{16} \mathrm{H}_{17} \mathrm{~F}_{5} \mathrm{O}_{4} \mathrm{Na}[\mathrm{M}+\mathrm{Na}]^{+}$: 391.0939 ; found: 391.0936 .

\section{Mechanistic studies:}

TEMPO trapping experiments (cf. Eqs. 4a and 4b).

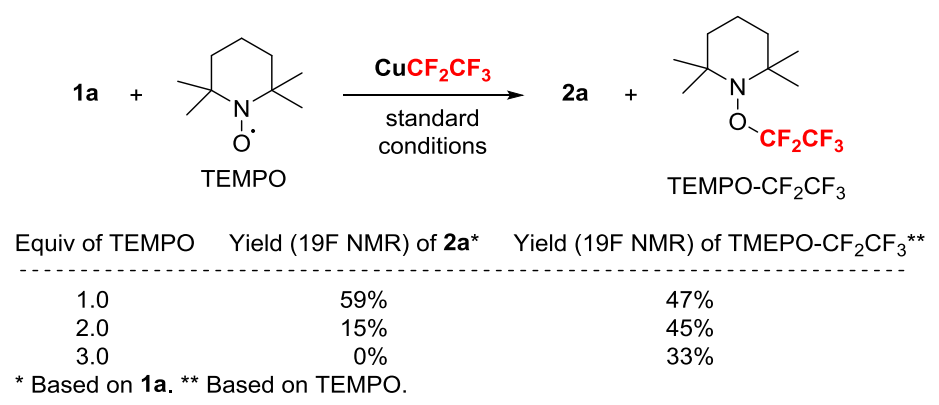

Under air, to a glass tube equipped with a magnetic stir bar was added alkene 1a (20.4 mg, 0.1 mmol), TEMPO (15.6 mg for 1.0 equiv; $31.2 \mathrm{mg}$ for 2.0 equiv; $46.8 \mathrm{mg}$ for 3.0 equiv), then freshly prepared $\left[\mathrm{CuCF}_{2} \mathrm{CF}_{3}\right](0.33 \mathrm{~mL}, 0.90 \mathrm{mmol}$ in DMF $)$ was added dropwise at $0{ }^{\circ} \mathrm{C}$. The tube was warmed to room temperature and stirred for $24 \mathrm{~h}$. The crude yield of each equation was analyzed by ${ }^{19} \mathrm{~F}$ NMR using benzotrifluoride as the internal standard. TEMPO- $\mathbf{C F}_{2} \mathbf{C F}_{3}:{ }^{19} \mathbf{F}$ NMR $\left(376 \mathrm{MHz}, \mathrm{CDCl}_{3}\right): \delta$ 84.80 (s, 3F), - 85.81 (s, 2F). HRMS m/z (APCI): calcd. for $\mathrm{C}_{11} \mathrm{H}_{19} \mathrm{~F}_{5} \mathrm{NO}[\mathrm{M}+\mathrm{H}]^{+}: 276.1381$; found: 276.1380. The spectral data are in full accordance with the literature report. ${ }^{5}$

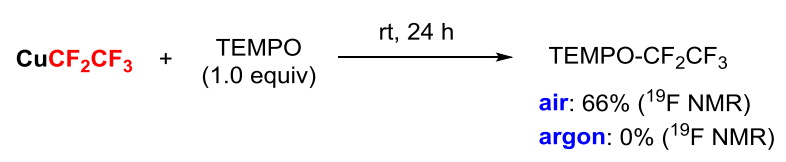

Under air or argon, to a glass tube equipped with a magnetic stir bar and TEMPO (46.8 $\mathrm{mg}, 0.3$ mmol) was added freshly prepared $\left[\mathrm{CuCF}_{2} \mathrm{CF}_{3}\right](0.33 \mathrm{~mL}, 0.90 \mathrm{mmol}$ in DMF $)$ dropwise at $0{ }^{\circ} \mathrm{C}$. Then the tube was warmed to room temperature and stirred for $24 \mathrm{~h}$. The crude yield of under air or argon condition was analyzed by ${ }^{19} \mathrm{~F}$ NMR using benzotrifluoride as the internal standard.

5 (a) Hartmann, M.; Li, Y.; Studer, A. Org. Biomol. Chem. 2016, 14, 206; (b) Xu, J.; Qiao, L.; Ying, B.; Zhu, X.; Shen, C.; Zhang, P. Org. Chem. Front. 2017, 4, 1116. 
Using $\mathrm{Cu}(\mathrm{OTf})_{2}$ and $\mathrm{TMSCF}_{2} \mathrm{CF}_{3}(c f$. Eq. 4c):

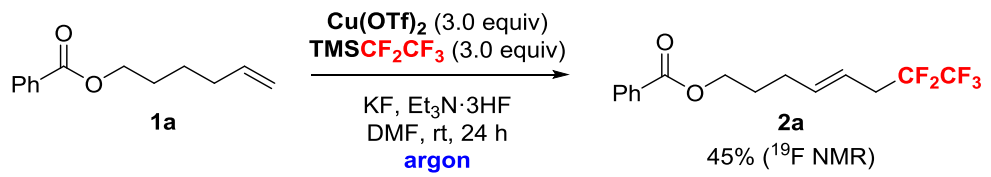

Under argon, to a glass tube equipped with a magnetic stir bar was added $\mathbf{1 a}(20.4 \mathrm{mg}, 0.1 \mathrm{mmol})$, $\mathrm{Cu}(\mathrm{OTf})_{2}(108.5 \mathrm{mg}, 0.3 \mathrm{mmol}), \mathrm{KF}(17.4 \mathrm{mg}, 0.3 \mathrm{mmol}), \mathrm{Et}_{3} \mathrm{~N} \cdot \mathrm{HF}(32.6 \mu \mathrm{L}, 0.2 \mathrm{mmol})$ and degassed DMF $(0.33 \mathrm{~mL})$, followed by addition of $\mathrm{TMSCF}_{2} \mathrm{CF}_{3}(53 \mu \mathrm{L}, 0.3 \mathrm{mmol})$ dropwise at $0{ }^{\circ} \mathrm{C}$. Then the tube was warmed to room temperature and stirred for $24 \mathrm{~h}$. The crude yield was analyzed by ${ }^{1} \mathrm{~F}$ NMR using benzotrifluoride as the internal standard.

\section{Radical clock experiments (cf. Eqs. $4 \mathrm{~d}$ and $4 \mathrm{e})$.}

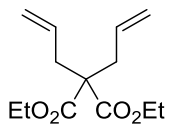

8

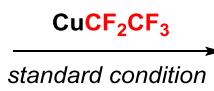

standard condition

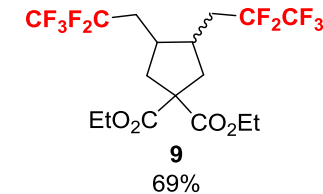

Under air, to a glass tube equipped with a magnetic stir bar and $8(72.0 \mathrm{mg}, 0.3 \mathrm{mmol})$ was added freshly prepared $\left[\mathrm{CuCF}_{2} \mathrm{CF}_{3}\right](1.0 \mathrm{~mL}, 0.90 \mathrm{mmol}$ in DMF $)$ dropwise at $0{ }^{\circ} \mathrm{C}$. Then the tube was warmed to room temperature and stirred for $24 \mathrm{~h}$. The reaction mixture was quenched with aq. sat. sodium potassium tartrate, extracted with diethyl ether three times. The organic layers were combined, washed with water then brine, dried over anhydrous $\mathrm{Na}_{2} \mathrm{SO}_{4}$, filtered and concentrated by rotary evaporator. The crude product was purified by flash column chromatography on silica gel (hexane/Et $\mathrm{E}_{2} \mathrm{O}$ ) to afford an inseparable mixture of $\mathbf{9}$ and $\mathbf{8}(\mathbf{9}: 0.21 \mathrm{mmol}, \mathrm{dr}=9: 1$, determined by GC MS; $8: 0.066 \mathrm{mmol}, \mathbf{9}+\mathbf{8}$ total $114 \mathrm{mg}, \mathbf{9}: \mathbf{8}=3.2: 1$ ) as a colorless oil. $\mathrm{R}_{\mathrm{f}}=0.5$ (hexane : EtOAc $=5: 1$ ). Compound 9 (major diasteromer): ${ }^{1} \mathbf{H}$ NMR (400 MHz, $\left.\mathrm{CDCl}_{3}\right): \delta 4.19-4.11(\mathrm{~m}, 4 \mathrm{H}), 2.55-2.49(\mathrm{~m}, 4 \mathrm{H}), 2.25-2.18(\mathrm{~m}, 2 \mathrm{H})$, 2.03-1.94 (m, 4H), 1.23-1.19 (m, 6H) ppm. ${ }^{13} \mathbf{C}$ NMR (101 MHz, $\left.\mathrm{CDCl}_{3}\right): \delta 172.5,172.0,119.1\left(\mathrm{qt}, J_{C-}\right.$ $\left.{ }_{F}=286.3 \mathrm{~Hz}, J_{C-F}=36.2 \mathrm{~Hz}\right), 115.9\left(\mathrm{tq}, J_{C-F}=254.0 \mathrm{~Hz}, J_{C-F}=37.7 \mathrm{~Hz}\right), 62.0,61.9,38.7,38.7,35.4$, $29.6\left(\mathrm{t}, J_{C-F}=21.6 \mathrm{~Hz}\right), 14.0,13.9 \mathrm{ppm} .{ }^{19} \mathbf{F}$ NMR $\left(376 \mathrm{MHz}, \mathrm{CDCl}_{3}\right): \delta-86.91(\mathrm{~s}, 3 \mathrm{~F}),-118.26(\mathrm{~m}, 2 \mathrm{~F})$. HRMS m/z (APCI): calcd. for $\mathrm{C}_{17} \mathrm{H}_{21} \mathrm{~F}_{10} \mathrm{O}_{4}[\mathrm{M}+\mathrm{H}]^{+}:$: 479.1275; found: 479.1275 .
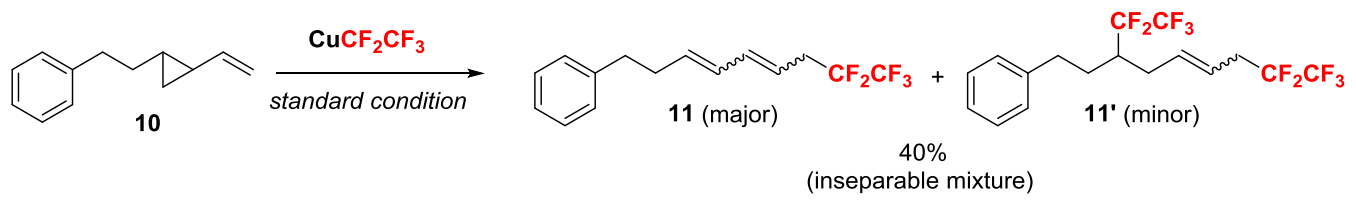

Under air, to a glass tube equipped with a magnetic stir bar and $\mathbf{1 0}(51.6 \mathrm{mg}, 0.3 \mathrm{mmol})$ was added freshly prepared $\left[\mathrm{CuCF}_{2} \mathrm{CF}_{3}\right](1.0 \mathrm{~mL}, 0.90 \mathrm{mmol}$ in $\mathrm{DMF})$ dropwise at $0{ }^{\circ} \mathrm{C}$. Then the tube was warmed to room temperature and stirred for $24 \mathrm{~h}$. The reaction mixture was quenched with aq. sat. sodium potassium tartrate, extracted with diethyl ether three times. The organic layers were combined, washed 
with water then brine, dried over anhydrous $\mathrm{Na}_{2} \mathrm{SO}_{4}$, filtered and concentrated by rotary evaporator. The crude product was purified by flash column chromatography on silica gel (hexane) to afford an inseparable mixture of $\mathbf{1 1}$ and $\mathbf{1 1}$ ' (total about $0.12 \mathrm{mmol}, 38 \mathrm{mg}$ ) as a colorless oil. $\mathrm{R}_{\mathrm{f}}=0.8$ (hexane : $\left.\mathrm{Et}_{2} \mathrm{O}=10: 1\right)$. Major isomer of 11: ${ }^{1} \mathbf{H}$ NMR $\left(400 \mathrm{MHz}, \mathrm{CDCl}_{3}\right): \delta 7.56(\mathrm{t}, J=7.4 \mathrm{~Hz}, 2 \mathrm{H}), 7.49-7.43$ (m, 3H), 6.53-6.43 (m, 1H), 6.39-6.28 (m, 1H), 6.06-5.99 (m, 1H), 5.79-5.72 (m, 1H), 3.11-3.04 (m, 2H), 3.01-2.97 (m, 2H), 2.69 (q, $J=7.7 \mathrm{~Hz}, 2 \mathrm{H})$ ppm. ${ }^{13} \mathbf{C}$ NMR (101 MHz, $\left.\mathrm{CDCl}_{3}\right): \delta 141.7,137.5,135.2$, $129.7,128.5,128.5,126.1,119.2\left(\mathrm{qt}, J_{C-F}=286.9 \mathrm{~Hz}, J_{C-F}=36.0 \mathrm{~Hz}\right), 114.9\left(\mathrm{tq}, J_{C-F}=253.2 \mathrm{~Hz}, J_{C-F}=\right.$ $36.4 \mathrm{~Hz}), 117.4\left(\mathrm{t}, J_{C-F}=17.6 \mathrm{~Hz}\right), 35.7,34.7\left(\mathrm{t}, J_{C-F}=22.6 \mathrm{~Hz}\right), 34.5 \mathrm{ppm} .{ }^{19} \mathbf{F} \mathbf{N M R}\left(376 \mathrm{MHz}, \mathrm{CDCl}_{3}\right)$ : $\delta$-85.97 (s, 3F), -118.22 (t, $J=17.7 \mathrm{~Hz}, 2 \mathrm{~F}$ ). HRMS m/z (APCI): calcd. for $\mathrm{C}_{15} \mathrm{H}_{16} \mathrm{~F}_{5}[\mathrm{M}+\mathrm{H}]^{+}: 291.1167$; found: 291.1167. Compound 11': HRMS m/z (ESI): calcd. for $\mathrm{C}_{17} \mathrm{H}_{17} \mathrm{~F}_{10}[\mathrm{M}+\mathrm{H}]^{+}:$: 411.1165; found: 411.1169 .

\section{Control experiments (cf. Eq. 4f).}

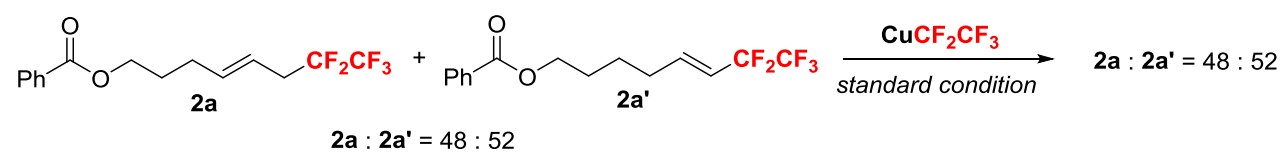

Under air, to a glass tube equipped with a magnetic stir bar and $\mathbf{2 a}$ and $\mathbf{2 a} \mathbf{a}^{\prime}(32.2 \mathrm{mg}, 0.1 \mathrm{mmol}, \mathbf{2 a}$ : $\left.\mathbf{2 a}{ }^{\prime}=48: 52\right)$ was added freshly prepared $\left[\mathrm{CuCF}_{2} \mathrm{CF}_{3}\right](0.33 \mathrm{~mL}, 0.90 \mathrm{mmol}$ in DMF $)$ dropwise at $0{ }^{\circ} \mathrm{C}$. The tube was warmed to room temperature and stirred for $24 \mathrm{~h}$. The crude $\mathbf{2 a} / \mathbf{2 a}$ ' ratio was analyzed by ${ }^{19} \mathrm{~F}$ NMR using benzotrifluoride as the internal standard.

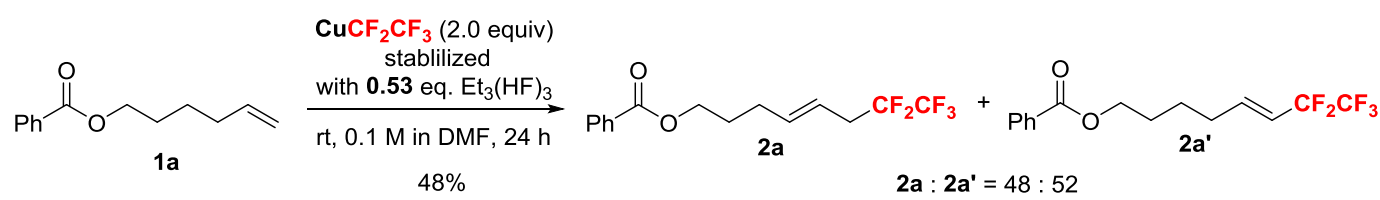

Preparation of 2a': Under air, to a glass tube equipped with a magnetic stir bar was added 1a (20.4 $\mathrm{mg}, 0.1 \mathrm{mmol})$ and DMF $(0.78 \mathrm{~mL})$. Then freshly prepared $\left[\mathrm{CuCF}_{2} \mathrm{CF}_{3}\right](0.22 \mathrm{~mL}, 0.90 \mathrm{mmol}$ in DMF, stabilized with 0.53 equiv $\mathrm{Et}_{3} \mathrm{~N} \cdot \mathrm{HF}$ ) was added dropwise at room temperature. The tube was stirred for $24 \mathrm{~h}$ at room temperature. Quenched with aq. sat. sodium potassium tartrate, extracted with diethyl ether three times. The organic layers were combined, washed with water then brine, dried over anhydrous $\mathrm{Na}_{2} \mathrm{SO}_{4}$, filtered and concentrated by rotary evaporator. The crude product was purified by flash column chromatography on silica gel (hexane/ $\mathrm{Et}_{2} \mathrm{O}$ ) to afford an inseparable mixture of $\mathbf{2 a}$ and $\mathbf{2 a}$ ' $(15.4 \mathrm{mg}$, 0.048 mmol, 2a : 2a' $=48: 52, \mathrm{R}_{\mathrm{f}}=0.6\left(\right.$ hexane $: \mathrm{Et}_{2} \mathrm{O}=8: 1$ )) as a colorless oil. Compound 2a': ${ }^{1} \mathbf{H}$ NMR $\left(400 \mathrm{MHz}, \mathrm{CDCl}_{3}\right): \delta 8.05$ (d, $\left.J=7.5 \mathrm{~Hz}, 2 \mathrm{H}\right), 7.55(\mathrm{t}, J=7.5 \mathrm{~Hz}, 1 \mathrm{H}), 7.44(\mathrm{t}, J=7.5 \mathrm{~Hz}, 2 \mathrm{H})$, 6.47-6.39 (m, 1H), 5.67-5.57 (m, 1H), $4.34(\mathrm{t}, J=6.4 \mathrm{~Hz}, 2 \mathrm{H}), 2.30-2.22(\mathrm{~m}, 2 \mathrm{H}), 1.82-1.77(\mathrm{~m}, 2 \mathrm{H})$, 1.67-1.59 (m, 2H) ppm. ${ }^{13} \mathbf{C}$ NMR $\left(101 \mathrm{MHz}, \mathrm{CDCl}_{3}\right): \delta 166.7,142.5\left(\mathrm{t}, J_{C-F}=8.7 \mathrm{~Hz}\right), 133.0,130.5$, $129.6,128.5,119.1$ (qt, $\left.J_{C-F}=286.3 \mathrm{~Hz}, J_{C-F}=38.5 \mathrm{~Hz}\right), 117.2\left(\mathrm{t}, J_{C-F}=23.2 \mathrm{~Hz}\right), 112.2\left(\mathrm{tq}, J_{C-F}=250.7\right.$ $\left.\mathrm{Hz}, J_{C-F}=38.5 \mathrm{~Hz}\right), 64.5,31.6,28.2,24.7 \mathrm{ppm} .{ }^{19} \mathbf{F} \mathbf{~ N M R}\left(376 \mathrm{MHz}, \mathrm{CDCl}_{3}\right): E$ isomer: $\delta-86.53(\mathrm{~s}, 3 \mathrm{~F})$, $-116.24(\mathrm{~d}, J=11.7 \mathrm{~Hz}, 2 \mathrm{~F})$. HRMS m/z (APCI): calcd. for $\mathrm{C}_{15} \mathrm{H}_{16} \mathrm{~F}_{5} \mathrm{O}_{2}[\mathrm{M}+\mathrm{H}]^{+}: 323.1065$; found: 323.1066 . 
Using D-labeled substrate (cf. Eq. 4g):

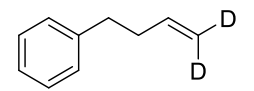

12

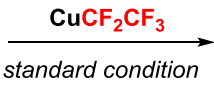

standard condition

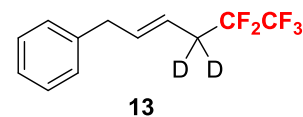

Under air, to a glass tube equipped with a magnetic stir bar and alkene $12(40.2 \mathrm{mg}, 0.3 \mathrm{mmol}, 92 \%$ D incorporation) was added freshly prepared $\left[\mathrm{CuCF}_{2} \mathrm{CF}_{3}\right]\left(1.0 \mathrm{~mL}, 0.90 \mathrm{mmol}\right.$ in DMF) at $0{ }^{\circ} \mathrm{C}$. Then was warmed to room temperature and stirred for $24 \mathrm{~h}$. Quenched with aq. sat. sodium potassium tartrate, extracted with diethyl ether three times. The organic layers were combined, washed with water then brine, dried over anhydrous $\mathrm{Na}_{2} \mathrm{SO}_{4}$, filtered and concentrated by rotary evaporator. The crude residue was purified by flash column chromatography on silica gel (hexane) to afford the desired product $\mathbf{1 3}(0.19$ mmol, $46.9 \mathrm{mg}, 90 \%$ D incorporation, $\mathrm{R}_{\mathrm{f}}=0.6$ (hexane)) as a colorless oil. Compound 13: ${ }^{1} \mathrm{H}$ NMR $\left(400 \mathrm{MHz}, \mathrm{CDCl}_{3}\right): \delta 7.40$ (t, $\left.J=7.4 \mathrm{~Hz}, 2 \mathrm{H}\right), 7.27-7.20(\mathrm{~m}, 3 \mathrm{H}), 5.90(\mathrm{dt}, J=15.2 \mathrm{~Hz}, J=6.8 \mathrm{~Hz}, 1 \mathrm{H})$, $5.51(\mathrm{~d}, J=15.2 \mathrm{~Hz}, 1 \mathrm{H}), 3.44(\mathrm{~d}, J=6.8 \mathrm{~Hz}, 2 \mathrm{H}) \mathrm{ppm} .{ }^{13} \mathbf{C} \mathbf{~ N M R}\left(101 \mathrm{MHz}, \mathrm{CDCl}_{3}\right): \delta 139.6,137.4$, $128.7,126.4,119.3$ (qt, $J_{C-F}=286.6 \mathrm{~Hz}, J_{C-F}=36.3 \mathrm{~Hz}$ ), $118.1,114.9$ (tq, $J_{C-F}=252.8 \mathrm{~Hz}, J_{C-F}=36.7$ $\mathrm{Hz}), 39.1 \mathrm{ppm}$. (one carbon missing due to overlap) ${ }^{19} \mathbf{F}$ NMR $\left(376 \mathrm{MHz}, \mathrm{CDCl}_{3}\right): \delta-85.94(\mathrm{~s}, 3 \mathrm{~F}),-$ 118.56 (s, 2F). HRMS m/z (APCI): calcd. for $\mathrm{C}_{12} \mathrm{H}_{9} \mathrm{D}_{2} \mathrm{~F}_{5}[\mathrm{M}]^{+}: 253.0979$; found: 253.0982 . 
Table S1. Optimization studies ${ }^{\mathrm{a}}$

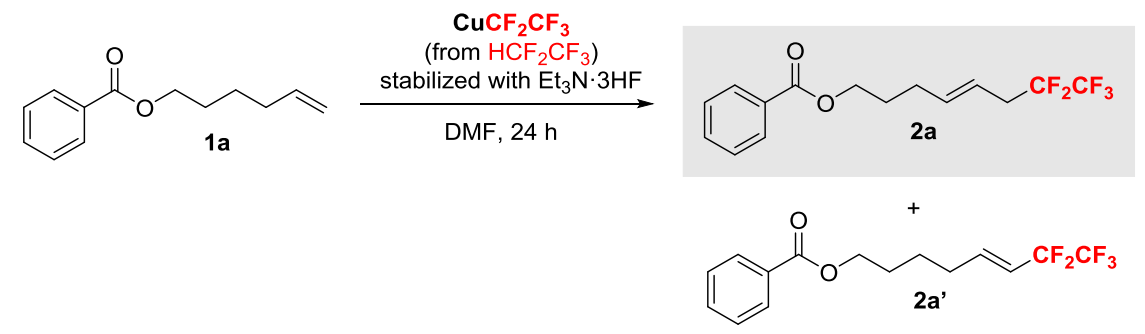

\begin{tabular}{|c|c|c|c|c|c|c|}
\hline entry & $\begin{array}{l}\text { equiv of } \\
\mathrm{Et}_{3} \mathrm{~N} \cdot 3 \mathrm{HF} \\
\text { (based on } \mathrm{Cu} \text { ) }\end{array}$ & $\begin{array}{l}\text { equiv of } \\
\mathrm{CuCF}_{2} \mathrm{CF}_{3}\end{array}$ & oxidant (equiv) & $\begin{array}{l}\text { conc. } \\
\text { (M) }\end{array}$ & $\begin{array}{l}\text { yield of } \\
\mathbf{2} \mathbf{a}+\mathbf{2} \mathbf{a}^{\prime}(\%)^{b}\end{array}$ & $\begin{array}{l}\text { ratio of } \\
\mathbf{2} \mathbf{a}: \mathbf{2} \mathbf{a}^{\prime b}\end{array}$ \\
\hline 1 & 0.53 & 2.0 & air & 0.1 & 59 & $1: 1.3$ \\
\hline 2 & 0.75 & 2.0 & air & 0.1 & 59 & $5.5: 1$ \\
\hline 3 & 1.0 & 2.0 & air & 0.1 & 59 & 19:1 \\
\hline 4 & 1.2 & 2.0 & air & 0.1 & 42 & 19:1 \\
\hline 5 & 1.0 & 2.0 & air & 0.2 & 67 & $21: 1$ \\
\hline 6 & 1.0 & 2.0 & air & 0.3 & 69 & $22: 1$ \\
\hline 7 & 1.0 & 2.0 & air & 0.4 & 71 & $22: 1$ \\
\hline $8^{c}$ & 1.0 & 2.0 & air & 0.4 & 78 & $21: 1$ \\
\hline $9^{\mathrm{c}, \mathrm{d}}$ & 1.0 & 2.0 & air & 0.4 & 56 & $25: 1$ \\
\hline $10^{\mathrm{c}, \mathrm{e}}$ & 1.0 & 2.0 & none & 0.4 & $<5$ & - \\
\hline $11^{\mathrm{c}, \mathrm{e}}$ & 1.0 & 2.0 & $\mathrm{AgOAc}(2.0)$ & 0.4 & 37 & $14: 1$ \\
\hline $12^{\mathrm{c}, \mathrm{e}}$ & 1.0 & 2.0 & $\mathrm{PhI}(\mathrm{OAc})_{2}(2.0)$ & 0.4 & 77 & 11:1 \\
\hline $13^{\mathrm{c}, \mathrm{e}}$ & 1.0 & 2.0 & DDQ (2.0) & 0.4 & $<5$ & - \\
\hline $14^{\mathrm{c}, \mathrm{e}}$ & 1.0 & 2.0 & $\begin{array}{l}\text { 1,4-Benzoquinone } \\
(2.0)\end{array}$ & 0.4 & $<5$ & - \\
\hline $15^{\mathrm{c}, \mathrm{e}}$ & 1.0 & 2.0 & $\begin{array}{l}\text { Di-tert-butyl } \\
\text { peroxide }(2.0)\end{array}$ & 0.4 & 28 & $27: 1$ \\
\hline $16^{\mathrm{c}}$ & 1.0 & 2.0 & $\mathrm{O}_{2}$ balloon & 0.4 & 46 & $15: 1$ \\
\hline $17^{\mathrm{c}, \mathrm{f}}$ & 1.0 & 2.0 & air & 0.4 & 64 & $16: 1$ \\
\hline $18^{\mathrm{c}}$ & 1.0 & 1.5 & air & 0.4 & 68 & 19:1 \\
\hline $19^{c}$ & 1.0 & 3.0 & air & 0.3 & $88(87)^{\mathrm{g}}$ & 20:1 \\
\hline $20^{c}$ & 1.0 & 4.0 & air & 0.23 & 87 & $16: 1$ \\
\hline
\end{tabular}

${ }^{\mathrm{a}}$ Unless specified otherwise, reactions were carried out using $0.1 \mathrm{mmol} \mathbf{1 a}$ at room temperature open to air. ${ }^{b}$ Determined by ${ }^{1} \mathrm{H}$ NMR analysis of the crude mixture. ${ }^{\circ} \mathrm{CuCF}_{2} \mathrm{CF}_{3}$ was added at $0{ }^{\circ} \mathrm{C}$ then the reaction mixture was warmed to room temperature. ${ }^{\mathrm{d}} \mathrm{CuCF}_{2} \mathrm{CF}_{3}$ was stabilized with 0.33 equiv Olah's reagent (HF-pyridine). ${ }^{\mathrm{e}}$ Under argon. ${ }^{\mathrm{f}} \mathrm{At} 50^{\circ} \mathrm{C}$. IIsolated yield at $0.3 \mathrm{mmol}$ scale $\left(\mathbf{2 a}: 2 \mathbf{a}^{\prime}=20: 1 ; E / Z\right.$ of $\mathbf{2 a}=92: 8)$. 


\section{Characterization Data:}<smiles>O=C(OCCC=CCC(F)(F)F)c1ccccc1</smiles>

2a: (E)-7,7,8,8,8-pentafluorooct-4-en-1-yl benzoate. Prepared according to the general procedure. Reaction was run using $1 \mathbf{a}(61.2 \mathrm{mg}, 0.3 \mathrm{mmol}),\left[\mathrm{CuCF}_{2} \mathrm{CF}_{3}\right](1.0 \mathrm{~mL}, 0.90 \mathrm{mmol}$ in DMF). The product was purified by flash column chromatography on silica gel (hexane/Et $\mathrm{t}_{2} \mathrm{O}$ ) and obtained a colorless oil $(0.26 \mathrm{mmol}, 84.0 \mathrm{mg}, 87 \%, E / Z=92: 8$, allylic : vinylic $=20: 1), \mathrm{R}_{\mathrm{f}}=0.6\left(\right.$ hexane $\left.: \mathrm{Et}_{2} \mathrm{O}=8: 1\right) .{ }^{1} \mathrm{H}$ NMR $\left(500 \mathrm{MHz}, \mathrm{CDCl}_{3}\right): \delta 8.05(\mathrm{~d}, J=7.5 \mathrm{~Hz}, 2 \mathrm{H}), 7.55(\mathrm{t}, J=7.5 \mathrm{~Hz}, 1 \mathrm{H}), 7.44(\mathrm{t}, J=7.5 \mathrm{~Hz}, 2 \mathrm{H})$, 5.78-5.72 (m, 1H), 5.49-5.43 (m, 1H), $4.33(\mathrm{t}, J=6.5 \mathrm{~Hz}, 2 \mathrm{H}), 2.74$ (td, $J=17.6 \mathrm{~Hz}, J=7.5 \mathrm{~Hz}, 2 \mathrm{H})$, 2.24 (q, $J=7.2 \mathrm{~Hz}, 2 \mathrm{H}), 1.87$ (m, 2H) ppm. ${ }^{13} \mathbf{C}$ NMR $\left(126 \mathrm{MHz}, \mathrm{CDCl}_{3}\right): \delta 166.6,137.4,133.0,130.4$, $129.6,128.4,119.3\left(\mathrm{qt}, J_{C-F}=286.1 \mathrm{~Hz}, J_{C-F}=36.3 \mathrm{~Hz}\right), 117.7\left(\mathrm{t}, J_{C-F}=4.3 \mathrm{~Hz}\right), 114.8\left(\mathrm{tq}, J_{C-F}=252.3\right.$ $\left.\mathrm{Hz}, J_{C-F}=37.0 \mathrm{~Hz}\right), 64.2,34.6\left(\mathrm{t}, J_{C-F}=22.5 \mathrm{~Hz}\right), 29.1,28.1 \mathrm{ppm} .{ }^{19} \mathbf{F}$ NMR $\left(470 \mathrm{MHz}, \mathrm{CDCl}_{3}\right): E$ isomer: $\delta-85.93$ (s, 3F), -118.37 (t, $J=17.4 \mathrm{~Hz}, 2 \mathrm{~F}) ; Z$ isomer: $\delta-86.16(\mathrm{~s}, 3 \mathrm{~F}),-118.21$ (t, $J=17.9 \mathrm{~Hz}, 2 \mathrm{~F})$. HRMS m/z (ESI): calcd. for $\mathrm{C}_{15} \mathrm{H}_{15} \mathrm{~F}_{5} \mathrm{O}_{2} \mathrm{Na}[\mathrm{M}+\mathrm{Na}]^{+}:$345.0884; found: 345.0881 .

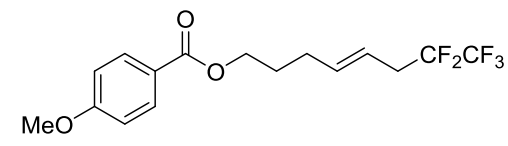

2b: (E)-7,7,8,8,8-pentafluorooct-4-en-1-yl 4-methoxybenzoate. Prepared according to the general procedure. Reaction was run using $\mathbf{1 b}(70.2 \mathrm{mg}, 0.3 \mathrm{mmol})$, [ $\left.\mathrm{CuCF}_{2} \mathrm{CF}_{3}\right](1.0 \mathrm{~mL}, 0.90 \mathrm{mmol}$ in DMF). The product was purified by flash column chromatography on silica gel (hexane/Et $\mathrm{E}_{2} \mathrm{O}$ ) and obtained a colorless oil $(0.26 \mathrm{mmol}, 89.8 \mathrm{mg}, 85 \%, E / Z=91: 9$, allylic : vinylic $=23: 1), \mathrm{R}_{\mathrm{f}}=0.5$ (hexane $: \mathrm{Et}_{2} \mathrm{O}$ $=5: 1) .{ }^{1} \mathbf{H}$ NMR $\left(400 \mathrm{MHz}, \mathrm{CDCl}_{3}\right): \delta 7.99(\mathrm{~d}, J=8.4 \mathrm{~Hz}, 2 \mathrm{H}), 6.91(\mathrm{~d}, J=8.4 \mathrm{~Hz}, 2 \mathrm{H}), 5.78-5.70(\mathrm{~m}$, 1H), 5.48-5.41 (m, 1H), 4.29 (t, $J=6.6 \mathrm{~Hz}, 2 \mathrm{H}), 3.83$ (d, $J=1.2 \mathrm{~Hz}, 3 \mathrm{H}), 2.74(\mathrm{td}, J=17.6 \mathrm{~Hz}, J=7.2$ $\mathrm{Hz}, 2 \mathrm{H}), 2.22$ (q, $J=7.2 \mathrm{~Hz}, 2 \mathrm{H}), 1.84(\mathrm{~m}, 2 \mathrm{H}) \mathrm{ppm} .{ }^{13} \mathbf{C} \mathbf{N M R}\left(101 \mathrm{MHz}, \mathrm{CDCl}_{3}\right): \delta 166.4,163.5$, $137.5,131.6,122.9,119.2\left(\mathrm{qt}, J_{C-F}=286.6 \mathrm{~Hz}, J_{C-F}=36.4 \mathrm{~Hz}\right), 117.6\left(\mathrm{t}, J_{C-F}=4.3 \mathrm{~Hz}\right), 114.8\left(\mathrm{tq}, J_{C-F}=\right.$ $\left.252.7 \mathrm{~Hz}, J_{C-F}=37.2 \mathrm{~Hz}\right), 113.7,63.9,55.4,34.6\left(\mathrm{t}, J_{C-F}=22.5 \mathrm{~Hz}\right), 29.1,28.2 \mathrm{ppm} .{ }^{19} \mathbf{F}$ NMR $(470$ $\mathrm{MHz}, \mathrm{CDCl}_{3}$ ): $E$ isomer: $\delta-85.78(\mathrm{~s}, 3 \mathrm{~F}),-118.23(\mathrm{t}, J=17.4 \mathrm{~Hz}, 2 \mathrm{~F}) ; Z$ isomer: $\delta-85.99$ (s, 3F), -118.05 (t, $J=17.9 \mathrm{~Hz}, 2 \mathrm{~F}$ ). HRMS m/z (ESI): calcd. for $\mathrm{C}_{16} \mathrm{H}_{17} \mathrm{~F}_{5} \mathrm{O}_{3} \mathrm{Na}[\mathrm{M}+\mathrm{Na}]^{+}:$375.0990; found: 375.0987 .<smiles>N#Cc1ccc(C(=O)OCCC/C=C/CC(F)(F)F)cc1</smiles>

2c: (E)-7,7,8,8,8-pentafluorooct-4-en-1-yl 4-cyanobenzoate. Prepared according to the general procedure. Reaction was run using $1 \mathbf{c}(68.7 \mathrm{mg}, 0.3 \mathrm{mmol}),\left[\mathrm{CuCF}_{2} \mathrm{CF}_{3}\right](1.0 \mathrm{~mL}, 0.90 \mathrm{mmol}$ in DMF). The product was purified by flash column chromatography on silica gel (hexane/ $\mathrm{Et}_{2} \mathrm{O}$ ) and obtained a colorless oil $(0.23 \mathrm{mmol}, 79.1 \mathrm{mg}, 76 \%, E / Z=92: 8$, allylic : vinylic $>50: 1), \mathrm{R}_{\mathrm{f}}=0.4$ (hexane : $\mathrm{Et}_{2} \mathrm{O}$ $=5: 1) .{ }^{1} \mathbf{H}$ NMR $\left(500 \mathrm{MHz}, \mathrm{CDCl}_{3}\right): \delta 8.12(\mathrm{~d}, J=8.0 \mathrm{~Hz}, 2 \mathrm{H}), 7.73(\mathrm{~d}, J=8.0 \mathrm{~Hz}, 2 \mathrm{H}), 5.76-5.70(\mathrm{~m}$, $1 \mathrm{H}), 5.48-5.42(\mathrm{~m}, 1 \mathrm{H}), 4.34$ (t, $J=6.5 \mathrm{~Hz}, 2 \mathrm{H}), 2.73$ (td, $J=17.6 \mathrm{~Hz}, J=7.0 \mathrm{~Hz}, 2 \mathrm{H}), 2.23$ (q, $J=7.2$ $\mathrm{Hz}, 2 \mathrm{H}), 1.87$ (m, 2H) ppm. ${ }^{13} \mathbf{C}$ NMR $\left(126 \mathrm{MHz}, \mathrm{CDCl}_{3}\right): \delta 165.0,137.1,134.2,132.3,130.1,119.3$ (qt, $\left.J_{C-F}=286.1 \mathrm{~Hz}, J_{C-F}=36.2 \mathrm{~Hz}\right), 118.0,117.9\left(\mathrm{t}, J_{C-F}=4.2 \mathrm{~Hz}\right), 116.4,114.8\left(\mathrm{tq}, J_{C-F}=252.5 \mathrm{~Hz}, J_{C-F}=\right.$ $37.0 \mathrm{~Hz}), 65.0,34.5\left(\mathrm{t}, J_{C-F}=22.4 \mathrm{~Hz}\right), 29.0,27.9 \mathrm{ppm} .{ }^{19} \mathbf{F} \mathbf{~ N M R}\left(470 \mathrm{MHz}, \mathrm{CDCl}_{3}\right): E$ isomer: $\delta-85.78$ 
(s, 3F), -118.22 (t, $J=17.4 \mathrm{~Hz}, 2 \mathrm{~F})$; $Z$ isomer: $\delta-86.01(\mathrm{~s}, 3 \mathrm{~F}),-118.05(\mathrm{t}, J=17.9 \mathrm{~Hz}, 2 \mathrm{~F})$. HRMS m/z (APCI): calcd. for $\mathrm{C}_{16} \mathrm{H}_{15} \mathrm{~F}_{5} \mathrm{NO}_{2}[\mathrm{M}+\mathrm{H}]^{+}$: 348.1018; found: 348.1017 .

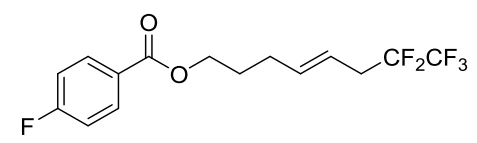

2d: (E)-7,7,8,8,8-pentafluorooct-4-en-1-yl 4-fluorobenzoate. Prepared according to the general procedure. Reaction was run using $\mathbf{1 d}(66.6 \mathrm{mg}, 0.3 \mathrm{mmol}),\left[\mathrm{CuCF}_{2} \mathrm{CF}_{3}\right]$ (1.0 mL, $0.90 \mathrm{mmol}$ in DMF). The product was purified by flash column chromatography on silica gel (hexane/Et ${ }_{2} \mathrm{O}$ ) and obtained a colorless oil $(0.24 \mathrm{mmol}, 82.6 \mathrm{mg}, 81 \%, E / Z=92: 8$, allylic : vinylic $=24: 1), \mathrm{R}_{\mathrm{f}}=0.6\left(\right.$ hexane $: \mathrm{Et}_{2} \mathrm{O}$ $=8: 1) .{ }^{1} \mathbf{H}$ NMR (500 MHz, $\left.\mathrm{CDCl}_{3}\right): \delta 8.06-8.03(\mathrm{~m}, 2 \mathrm{H}), 7.73(\mathrm{t}, J=8.0 \mathrm{~Hz}, 2 \mathrm{H}), 5.77-5.71(\mathrm{~m}, 1 \mathrm{H})$, 5.48-5.42 (m, 1H), $4.31(\mathrm{t}, J=6.3 \mathrm{~Hz}, 2 \mathrm{H}), 2.74(\mathrm{td}, J=17.5 \mathrm{~Hz}, J=7.0 \mathrm{~Hz}, 2 \mathrm{H}), 2.23(\mathrm{q}, J=7.2 \mathrm{~Hz}$, 2H), $1.86(\mathrm{~m}, 2 \mathrm{H})$ ppm. ${ }^{13} \mathbf{C}$ NMR $\left(101 \mathrm{MHz}, \mathrm{CDCl}_{3}\right): \delta 165.9\left(\mathrm{~d}, J_{C-F}=254.8 \mathrm{~Hz}\right), 165.7,137.4,132.2$ $\left(\mathrm{d}, J_{C-F}=9.3 \mathrm{~Hz}\right), 126.7\left(\mathrm{~d}, J_{C-F}=2.9 \mathrm{~Hz}\right), 119.3\left(\mathrm{qt}, J_{C-F}=286.6 \mathrm{~Hz}, J_{C-F}=36.3 \mathrm{~Hz}\right), 117.8\left(\mathrm{t}, J_{C-F}=4.5\right.$ $\mathrm{Hz}), 115.6\left(\mathrm{~d}, J_{C-F}=22.1 \mathrm{~Hz}\right), 114.9\left(\mathrm{tq}, J_{C-F}=252.9 \mathrm{~Hz}, J_{C-F}=37.2 \mathrm{~Hz}\right), 64.4,34.6\left(\mathrm{t}, J_{C-F}=22.5 \mathrm{~Hz}\right)$, 29.1, 28.1 ppm. ${ }^{19} \mathbf{F}$ NMR (470 MHz, $\left.\mathrm{CDCl}_{3}\right): E$ isomer: $\delta-85.78(\mathrm{~s}, 3 \mathrm{~F}),-106.79(\mathrm{~m}, 1 \mathrm{~F}),-118.22(\mathrm{t}, J$ $=17.4 \mathrm{~Hz}, 2 \mathrm{~F}) ; Z$ isomer: $\delta-86.01(\mathrm{~s}, 3 \mathrm{~F}),-106.71(\mathrm{~m}, 1 \mathrm{~F}),-118.06(\mathrm{t}, J=17.7 \mathrm{~Hz}, 2 \mathrm{~F}) . \mathbf{H R M S ~ m} / \mathrm{z}$ (APCI): calcd. for $\mathrm{C}_{15} \mathrm{H}_{15} \mathrm{~F}_{6} \mathrm{O}_{2}[\mathrm{M}+\mathrm{H}]^{+}: 341.0971$; found: 341.0971 .<smiles>O=C(OCCC/C=C/CC(F)(F)F)c1ccc(Cl)cc1</smiles>

2e: (E)-7,7,8,8,8-pentafluorooct-4-en-1-yl 4-chlorobenzoate. Prepared according to the general procedure. Reaction was run using 1 e $(71.4 \mathrm{mg}, 0.3 \mathrm{mmol}),\left[\mathrm{CuCF}_{2} \mathrm{CF}_{3}\right]$ (1.0 mL, $0.90 \mathrm{mmol}$ in DMF). The product was purified by flash column chromatography on silica gel (hexane/Et ${ }_{2} \mathrm{O}$ ) and obtained a colorless oil $(0.25 \mathrm{mmol}, 87.6 \mathrm{mg}, 82 \%, E / Z=92: 8$, allylic : vinylic $=48: 1), \mathrm{R}_{\mathrm{f}}=0.6$ (hexane : $\mathrm{Et}_{2} \mathrm{O}$ $=8: 1) .{ }^{1} \mathbf{H}$ NMR $\left(500 \mathrm{MHz}, \mathrm{CDCl}_{3}\right): \delta 7.96(\mathrm{~d}, J=8.0 \mathrm{~Hz}, 2 \mathrm{H}), 7.40(\mathrm{~d}, J=8.0 \mathrm{~Hz}, 2 \mathrm{H}), 5.77-5.71(\mathrm{~m}$, $1 \mathrm{H}), 5.48-5.42(\mathrm{~m}, 1 \mathrm{H}), 4.31(\mathrm{t}, J=6.5 \mathrm{~Hz}, 2 \mathrm{H}), 2.74(\mathrm{td}, J=17.5 \mathrm{~Hz}, J=7.0 \mathrm{~Hz}, 2 \mathrm{H}), 2.23(\mathrm{q}, J=7.2$ $\mathrm{Hz}, 2 \mathrm{H}), 1.86(\mathrm{~m}, 2 \mathrm{H}) \mathrm{ppm} .{ }^{13} \mathbf{C} \mathbf{N M R}\left(126 \mathrm{MHz}, \mathrm{CDCl}_{3}\right): \delta 165.8,139.5,137.3,131.0,128.9,128.8$, $119.2\left(\mathrm{qt}, J_{C-F}=286.1 \mathrm{~Hz}, J_{C-F}=36.4 \mathrm{~Hz}\right), 117.8\left(\mathrm{t}, J_{C-F}=4.3 \mathrm{~Hz}\right), 114.8\left(\mathrm{tq}, J_{C-F}=252.5 \mathrm{~Hz}, J_{C-F}=37.2\right.$ $\mathrm{Hz}), 64.5,34.6\left(\mathrm{t}, J_{C-F}=22.4 \mathrm{~Hz}\right), 29.1,28.0 \mathrm{ppm} .{ }^{19} \mathbf{F}$ NMR $\left(470 \mathrm{MHz}, \mathrm{CDCl}_{3}\right): E$ isomer: $\delta-85.79(\mathrm{~s}$, 3F), $-118.23(\mathrm{t}, J=17.4 \mathrm{~Hz}, 2 \mathrm{~F}) ; Z$ isomer: $\delta-86.00(\mathrm{~s}, 3 \mathrm{~F}),-118.10(\mathrm{t}, J=17.7 \mathrm{~Hz}, 2 \mathrm{~F})$. HRMS m/z (APCI): calcd. for $\mathrm{C}_{15} \mathrm{H}_{15} \mathrm{ClF}_{5} \mathrm{O}_{2}[\mathrm{M}+\mathrm{H}]^{+}: 357.0675$; found: 357.0674 .<smiles>O=C(OCCCC=CCC(F)(F)F)c1ccc(Br)cc1</smiles>

2f: (E)-7,7,8,8,8-pentafluorooct-4-en-1-yl 4-bromobenzoate. Prepared according to the general procedure. Reaction was run using $\mathbf{1 f}(84.6 \mathrm{mg}, 0.3 \mathrm{mmol}),\left[\mathrm{CuCF}_{2} \mathrm{CF}_{3}\right]$ (1.0 mL, $0.90 \mathrm{mmol}$ in DMF). The product was purified by flash column chromatography on silica gel (hexane/Et ${ }_{2} \mathrm{O}$ ) and obtained a colorless oil $(0.25 \mathrm{mmol}, 100.8 \mathrm{mg}, 84 \%, E / Z=92: 8$, allylic : vinylic $=24: 1), \mathrm{R}_{\mathrm{f}}=0.6$ (hexane $: \mathrm{Et}_{2} \mathrm{O}$ $=8: 1) .{ }^{1} \mathbf{H}$ NMR $\left(400 \mathrm{MHz}, \mathrm{CDCl}_{3}\right): \delta 7.88(\mathrm{~d}, J=8.4 \mathrm{~Hz}, 2 \mathrm{H}), 7.56(\mathrm{~d}, J=8.4 \mathrm{~Hz}, 2 \mathrm{H}), 5.77-5.70(\mathrm{~m}$, $1 \mathrm{H}), 5.49-5.41(\mathrm{~m}, 1 \mathrm{H}), 4.31(\mathrm{t}, J=6.4 \mathrm{~Hz}, 2 \mathrm{H}), 2.74(\mathrm{td}, J=17.6 \mathrm{~Hz}, J=7.2 \mathrm{~Hz}, 2 \mathrm{H}), 2.22(\mathrm{q}, J=7.1$ $\mathrm{Hz}, 2 \mathrm{H}), 1.86(\mathrm{~m}, 2 \mathrm{H}) \mathrm{ppm} .{ }^{13} \mathbf{C} \mathbf{N M R}\left(126 \mathrm{MHz}, \mathrm{CDCl}_{3}\right): \delta 165.9,137.3,131.8,131.2,129.3,128.1$, 
$119.2\left(\mathrm{qt}, J_{C-F}=286.1 \mathrm{~Hz}, J_{C-F}=36.3 \mathrm{~Hz}\right), 117.8\left(\mathrm{t}, J_{C-F}=4.2 \mathrm{~Hz}\right), 114.8\left(\mathrm{tq}, J_{C-F}=252.4 \mathrm{~Hz}, J_{C-F}=37.2\right.$ $\mathrm{Hz}), 64.5,34.6\left(\mathrm{t}, J_{C-F}=22.4 \mathrm{~Hz}\right), 29.1,28.0 \mathrm{ppm} .{ }^{19} \mathbf{F}$ NMR $\left(470 \mathrm{MHz}, \mathrm{CDCl}_{3}\right): E$ isomer: $\delta-85.78(\mathrm{~s}$, 3F), -118.23 (t, $J=17.7 \mathrm{~Hz}, 2 \mathrm{~F}) ; Z$ isomer: $\delta-86.00(\mathrm{~s}, 3 \mathrm{~F}),-118.05(\mathrm{t}, J=17.9 \mathrm{~Hz}, 2 \mathrm{~F}) . \mathbf{H R M S} \mathrm{m} / \mathrm{z}$ (APCI): calcd. for $\mathrm{C}_{15} \mathrm{H}_{15} \mathrm{BrF}_{5} \mathrm{O}_{2}[\mathrm{M}+\mathrm{H}]^{+}$: 401.0170; found: 401.0168 .<smiles>O=C(OCCC/C=C/CC(F)(F)F)c1ccc(I)cc1</smiles>

2g: (E)-7,7,8,8,8-pentafluorooct-4-en-1-yl 4-iodobenzoate. Prepared according to the general procedure. Reaction was run using $1 \mathrm{~g}(99.0 \mathrm{mg}, 0.3 \mathrm{mmol}),\left[\mathrm{CuCF}_{2} \mathrm{CF}_{3}\right]$ (1.0 mL, $0.90 \mathrm{mmol}$ in DMF). The product was purified by flash column chromatography on silica gel (hexane/Et ${ }_{2} \mathrm{O}$ ) and obtained a colorless oil $(0.22 \mathrm{mmol}, 96.8 \mathrm{mg}, 72 \%, E / Z=93: 7$, allylic : vinylic $>50: 1$, the product was contaminated by $\sim 5 \%$ cross-coupled side product), $\mathrm{R}_{\mathrm{f}}=0.6$ (hexane : $\left.\mathrm{Et}_{2} \mathrm{O}=8: 1\right) .{ }^{1} \mathbf{H} \mathbf{N M R}(500 \mathrm{MHz}$, $\left.\mathrm{CDCl}_{3}\right): \delta 7.79(\mathrm{~d}, J=8.5 \mathrm{~Hz}, 2 \mathrm{H}), 7.73(\mathrm{~d}, J=8.5 \mathrm{~Hz}, 2 \mathrm{H}), 5.77-5.71(\mathrm{~m}, 1 \mathrm{H}), 5.48-5.42(\mathrm{~m}, 1 \mathrm{H}), 4.31$ (t, $J=6.4 \mathrm{~Hz}, 2 \mathrm{H}), 2.74$ (td, $J=17.5 \mathrm{~Hz}, J=7.0 \mathrm{~Hz}, 2 \mathrm{H}), 2.22$ (q, $J=7.0 \mathrm{~Hz}, 2 \mathrm{H}), 1.86(\mathrm{~m}, 2 \mathrm{H}) \mathrm{ppm}$. ${ }^{13} \mathbf{C}$ NMR $\left(126 \mathrm{MHz}, \mathrm{CDCl}_{3}\right): \delta 166.2,137.8,137.3,131.1,129.9,119.2\left(\mathrm{qt}, J_{C-F}=286.3 \mathrm{~Hz}, J_{C-F}=36.2\right.$ $\mathrm{Hz}), 117.8\left(\mathrm{t}, J_{C-F}=4.2 \mathrm{~Hz}\right), 114.8\left(\mathrm{tq}, J_{C-F}=252.3 \mathrm{~Hz}, J_{C-F}=37.2 \mathrm{~Hz}\right), 100.8,64.5,34.6\left(\mathrm{t}, J_{C-F}=22.6\right.$ $\mathrm{Hz}), 29.1,28.0$ ppm. ${ }^{19} \mathbf{F}$ NMR $\left(470 \mathrm{MHz}, \mathrm{CDCl}_{3}\right): E$ isomer: $\delta-85.78(\mathrm{~s}, 3 \mathrm{~F}),-118.25(\mathrm{t}, J=17.7 \mathrm{~Hz}$, $2 \mathrm{~F}$ ); $Z$ isomer: $\delta-86.00(\mathrm{~s}, 3 \mathrm{~F}),-118.06(\mathrm{t}, J=17.9 \mathrm{~Hz}, 2 \mathrm{~F})$. HRMS m/z (APCI): calcd. for $\mathrm{C}_{15} \mathrm{H}_{15} \mathrm{IF}_{5} \mathrm{O}_{2}$ $[\mathrm{M}+\mathrm{H}]^{+}: 449.0031$; found: 449.0031 .

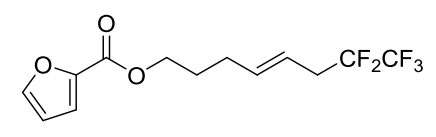

2h: (E)-7,7,8,8,8-pentafluorooct-4-en-1-yl furan-2-carboxylate. Prepared according to the general procedure. Reaction was run using $\mathbf{1 h}(58.2 \mathrm{mg}, 0.3 \mathrm{mmol}),\left[\mathrm{CuCF}_{2} \mathrm{CF}_{3}\right]$ (1.0 mL, $0.90 \mathrm{mmol}$ in DMF). The product was purified by flash column chromatography on silica gel (hexane/Et ${ }_{2} \mathrm{O}$ ) and obtained a colorless oil $(0.20 \mathrm{mmol}, 60.8 \mathrm{mg}, 65 \%, E / Z=92: 8$, allylic $:$ vinylic $=20: 1), \mathrm{R}_{\mathrm{f}}=0.6$ (hexane $: \mathrm{Et}_{2} \mathrm{O}$ $=8: 1) .{ }^{1} \mathbf{H}$ NMR $\left(400 \mathrm{MHz}, \mathrm{CDCl}_{3}\right): \delta 7.56(\mathrm{~s}, 1 \mathrm{H}), 7.15(\mathrm{~d}, J=3.6 \mathrm{~Hz}, 1 \mathrm{H}), 6.49(\mathrm{dd}, J=1.6 \mathrm{~Hz}, 2 \mathrm{H})$, 5.75-5.68 (m, 1H), 5.47-5.39 (m, 1H), 4.29 (t, $J=6.6 \mathrm{~Hz}, 2 \mathrm{H}), 2.72(\mathrm{td}, J=17.2 \mathrm{~Hz}, J=6.8 \mathrm{~Hz}, 2 \mathrm{H})$, $2.20(\mathrm{q}, J=7.1 \mathrm{~Hz}, 2 \mathrm{H}), 1.83(\mathrm{~m}, 2 \mathrm{H}) \mathrm{ppm} .{ }^{13} \mathbf{C} \mathbf{N M R}\left(126 \mathrm{MHz}, \mathrm{CDCl}_{3}\right): \delta 158.8,146.4,144.8,137.3$, $119.2\left(\mathrm{qt}, J_{C-F}=286.1 \mathrm{~Hz}, J_{C-F}=36.3 \mathrm{~Hz}\right), 117.9,117.8\left(\mathrm{t}, J_{C-F}=4.4 \mathrm{~Hz}\right), 114.8\left(\mathrm{tq}, J_{C-F}=252.4 \mathrm{~Hz}, J_{C-}\right.$ $\left.{ }_{F}=37.3 \mathrm{~Hz}\right), 111.9,64.2,34.6\left(\mathrm{t}, J_{C-F}=22.5 \mathrm{~Hz}\right), 29.0,28.0 \mathrm{ppm} .{ }^{19} \mathbf{F} \mathbf{N M R}\left(470 \mathrm{MHz}, \mathrm{CDCl}_{3}\right): E$ isomer: $\delta-85.78(\mathrm{~s}, 3 \mathrm{~F}),-118.23(\mathrm{t}, J=17.7 \mathrm{~Hz}, 2 \mathrm{~F}) ; Z$ isomer: $\delta-86.00(\mathrm{~s}, 3 \mathrm{~F}),-118.06(\mathrm{t}, J=17.7 \mathrm{~Hz}, 2 \mathrm{~F})$. HRMS m/z (ESI): calcd. for $\mathrm{C}_{13} \mathrm{H}_{13} \mathrm{~F}_{5} \mathrm{O}_{3} \mathrm{Na}[\mathrm{M}+\mathrm{Na}]^{+}: 335.0677$; found: 335.0673 .

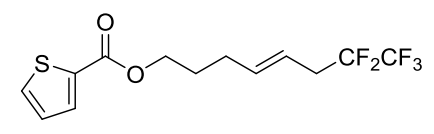

2i: (E)-7,7,8,8,8-pentafluorooct-4-en-1-yl thiophene-2-carboxylate. Prepared according to the general procedure. Reaction was run using $1 \mathbf{i}(63.0 \mathrm{mg}, 0.3 \mathrm{mmol}),\left[\mathrm{CuCF}_{2} \mathrm{CF}_{3}\right]$ (1.0 mL, $0.90 \mathrm{mmol}$ in DMF). The product was purified by flash column chromatography on silica gel (hexane/Et ${ }_{2} \mathrm{O}$ ) and obtained a colorless oil $(0.21 \mathrm{mmol}, 68.9 \mathrm{mg}, 70 \%, E / Z=92: 8$, allylic : vinylic $>50: 1), \mathrm{R}_{\mathrm{f}}=0.6$ (hexane : $\mathrm{Et}_{2} \mathrm{O}$ $=8: 1) .{ }^{1} \mathbf{H}$ NMR $\left(400 \mathrm{MHz}, \mathrm{CDCl}_{3}\right): \delta 7.80(\mathrm{~d}, J=3.6 \mathrm{~Hz}, 1 \mathrm{H}), 7.54(\mathrm{~d}, J=5.2 \mathrm{~Hz}, 1 \mathrm{H}), 7.09(\mathrm{t}, J=$ 
$4.0 \mathrm{~Hz}, 1 \mathrm{H}), 5.77-5.70(\mathrm{~m}, 1 \mathrm{H}), 5.49-5.42(\mathrm{~m}, 1 \mathrm{H}), 4.30(\mathrm{t}, J=6.4 \mathrm{~Hz}, 2 \mathrm{H}), 2.72(\mathrm{td}, J=17.6 \mathrm{~Hz}, J=$ $7.2 \mathrm{~Hz}, 2 \mathrm{H}), 2.22(\mathrm{q}, J=7.2 \mathrm{~Hz}, 2 \mathrm{H}), 1.84(\mathrm{~m}, 2 \mathrm{H}) \mathrm{ppm} .{ }^{13} \mathbf{C} \mathbf{N M R}\left(101 \mathrm{MHz}, \mathrm{CDCl}_{3}\right): \delta 162.3,137.4$, 134.0, 133.5, 132.4, 127.8, $119.3\left(\mathrm{qt}, J_{C-F}=286.5 \mathrm{~Hz}, J_{C-F}=36.4 \mathrm{~Hz}\right), 117.8\left(\mathrm{t}, J_{C-F}=4.4 \mathrm{~Hz}\right), 114.8$ (tq, $\left.J_{C-F}=252.4 \mathrm{~Hz}, J_{C-F}=37.3 \mathrm{~Hz}\right), 64.4,34.6\left(\mathrm{t}, J_{C-F}=22.5 \mathrm{~Hz}\right), 29.1,28.1 \mathrm{ppm} .{ }^{19} \mathbf{F} \mathbf{N M R}(470 \mathrm{MHz}$, $\left.\mathrm{CDCl}_{3}\right): E$ isomer: $\delta-85.78(\mathrm{~s}, 3 \mathrm{~F}),-118.23(\mathrm{t}, J=17.7 \mathrm{~Hz}, 2 \mathrm{~F}) ; Z$ isomer: $\delta-85.96(\mathrm{~s}, 3 \mathrm{~F}),-118.02(\mathrm{t}, J$ $=17.9 \mathrm{~Hz}, 2 \mathrm{~F})$. HRMS m/z (ESI): calcd. for $\mathrm{C}_{13} \mathrm{H}_{13} \mathrm{~F}_{5} \mathrm{O}_{2} \mathrm{SNa}[\mathrm{M}+\mathrm{Na}]^{+}:$351.0449; found: 351.0445 .<smiles>O=C(OCCC/C=C/CC(F)(F)F)c1ccccn1</smiles>

2j: (E)-7,7,8,8,8-pentafluorooct-4-en-1-yl picolinate. Prepared according to the general procedure. Reaction was run using $\mathbf{1 j}$ (61.5 mg, $0.3 \mathrm{mmol}),\left[\mathrm{CuCF}_{2} \mathrm{CF}_{3}\right]$ (1.0 mL, $0.90 \mathrm{mmol}$ in DMF). The product was purified by flash column chromatography on silica gel (hexane/EtOAc) and obtained a colorless oil (0.23 mmol, $72.7 \mathrm{mg}, 75 \%, E / Z=97: 3$, allylic : vinylic = 9: 1), $\mathrm{R}_{\mathrm{f}}=0.4$ (hexane : EtOAc $\left.=2: 1\right) .{ }^{1} \mathbf{H}$ NMR (400 MHz, $\left.\mathrm{CDCl}_{3}\right): \delta 8.73(\mathrm{~d}, J=4.0 \mathrm{~Hz}, 1 \mathrm{H}), 8.09(\mathrm{~d}, J=8.0 \mathrm{~Hz}, 1 \mathrm{H}), 7.81(\mathrm{t}, J=7.6 \mathrm{~Hz}, 1 \mathrm{H})$, $7.45(\mathrm{~m}, 1 \mathrm{H}), 5.75-5.68(\mathrm{~m}, 1 \mathrm{H}), 5.46-5.38(\mathrm{~m}, 1 \mathrm{H}), 4.39(\mathrm{t}, J=6.8 \mathrm{~Hz}, 2 \mathrm{H}), 2.71(\mathrm{td}, J=17.6 \mathrm{~Hz}, J=$ $7.2 \mathrm{~Hz}, 2 \mathrm{H}), 2.21(\mathrm{q}, J=7.2 \mathrm{~Hz}, 2 \mathrm{H}), 1.90(\mathrm{~m}, 2 \mathrm{H}) \mathrm{ppm} .{ }^{13} \mathbf{C} \mathbf{N M R}\left(126 \mathrm{MHz}, \mathrm{CDCl}_{3}\right): \delta 165.3,150.0$, 148.2, 137.3, 137.1, 127.0, 125.2, 119.2 (qt, $\left.J_{C-F}=286.1 \mathrm{~Hz}, J_{C-F}=36.4 \mathrm{~Hz}\right), 117.7\left(\mathrm{t}, J_{C-F}=4.3 \mathrm{~Hz}\right.$ ), $114.8\left(\mathrm{tq}, J_{C-F}=252.4 \mathrm{~Hz}, J_{C-F}=37.2 \mathrm{~Hz}\right), 65.2,34.6\left(\mathrm{t}, J_{C-F}=22.4 \mathrm{~Hz}\right), 29.0,28.0$ ppm. ${ }^{19} \mathbf{F}$ NMR $(470$ $\left.\mathrm{MHz}, \mathrm{CDCl}_{3}\right): E$ isomer: $\delta-85.79(\mathrm{~s}, 3 \mathrm{~F}),-118.25(\mathrm{t}, J=17.7 \mathrm{~Hz}, 2 \mathrm{~F}) ; Z$ isomer: $\delta-86.01(\mathrm{~s}, 3 \mathrm{~F}),-118.07$ (t, $J=17.4 \mathrm{~Hz}, 2 \mathrm{~F})$; vinyl isomer: -86.39 (s, 3F), -116.14 (d, $J=11.7 \mathrm{~Hz}, 2 \mathrm{~F})$. HRMS m/z (ESI): calcd. for $\mathrm{C}_{14} \mathrm{H}_{15} \mathrm{~F}_{5} \mathrm{NO}_{2}[\mathrm{M}+\mathrm{H}]^{+}$: 324.1018; found: 324.1012 .

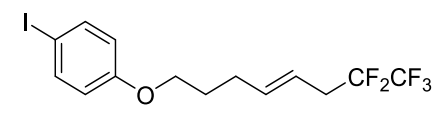

2k: (E)-1-iodo-4-((7,7,8,8,8-pentafluorooct-4-en-1-yl)oxy)benzene. Prepared according to the general procedure. Reaction was run using $1 \mathbf{k}(90.6 \mathrm{mg}, 0.3 \mathrm{mmol}),\left[\mathrm{CuCF}_{2} \mathrm{CF}_{3}\right]$ (1.0 mL, $0.90 \mathrm{mmol}$ in DMF). The product was purified by flash column chromatography on silica gel (hexane/Et $\mathrm{E}_{2} \mathrm{O}$ ) and obtained a colorless oil $(0.24 \mathrm{mmol}, 102.1 \mathrm{mg}, 81 \%, E / Z=91: 9$, allylic : vinylic $=20: 1), \mathrm{R}_{\mathrm{f}}=0.9$ (hexane $: \mathrm{Et}_{2} \mathrm{O}$ $=10$ : 1). ${ }^{1} \mathbf{H}$ NMR (400 MHz, $\left.\mathrm{CDCl}_{3}\right): \delta 7.55(\mathrm{~d}, J=8.0 \mathrm{~Hz}, 2 \mathrm{H}), 6.67(\mathrm{~d}, J=8.0 \mathrm{~Hz}, 2 \mathrm{H}), 5.79-5.72$ $(\mathrm{m}, 1 \mathrm{H}), 5.49-5.42(\mathrm{~m}, 1 \mathrm{H}), 3.92(\mathrm{t}, J=6.4 \mathrm{~Hz}, 2 \mathrm{H}), 2.76(\mathrm{td}, J=17.6 \mathrm{~Hz}, J=7.2 \mathrm{~Hz}, 2 \mathrm{H}), 2.26(\mathrm{q}, J=$ $7.1 \mathrm{~Hz}, 2 \mathrm{H}), 1.88(\mathrm{~m}, 2 \mathrm{H}) \mathrm{ppm} .{ }^{13} \mathbf{C}$ NMR (101 MHz, $\left.\mathrm{CDCl}_{3}\right): \delta 159.0,138.3,137.7,119.3\left(\mathrm{qt}, J_{C-F}=\right.$ $\left.286.6 \mathrm{~Hz}, J_{C-F}=36.3 \mathrm{~Hz}\right), 117.7\left(\mathrm{t}, J_{C-F}=4.4 \mathrm{~Hz}\right), 117.0,114.9\left(\mathrm{tq}, J_{C-F}=252.8 \mathrm{~Hz}, J_{C-F}=37.2 \mathrm{~Hz}\right)$, 82.7, 67.1, $34.7\left(\mathrm{t}, J_{C-F}=22.5 \mathrm{~Hz}\right), 29.0,28.5 \mathrm{ppm} .{ }^{19} \mathbf{F} \mathbf{N M R}\left(470 \mathrm{MHz}, \mathrm{CDCl}_{3}\right): E$ isomer: $\delta-85.76(\mathrm{~s}$, 3F), $-118.22(\mathrm{t}, J=17.7 \mathrm{~Hz}, 2 \mathrm{~F})$; $Z$ isomer: $\delta-85.97(\mathrm{~s}, 3 \mathrm{~F}),-118.03(\mathrm{t}, J=17.9 \mathrm{~Hz}, 2 \mathrm{~F}) . \mathbf{H R M S} \mathrm{m} / \mathrm{z}$ (ESI): calcd. for $\mathrm{C}_{14} \mathrm{H}_{14} \mathrm{~F}_{5} \mathrm{IO}[\mathrm{M}]^{+}: 420.0004$; found: 420.0000 .

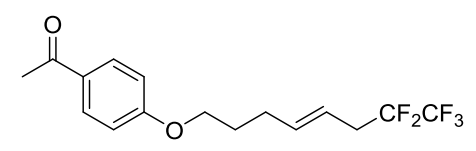

21: (E)-1-(4-((7,7,8,8,8-pentafluorooct-4-en-1-yl)oxy)phenyl)ethan-1-one. Prepared according to the general procedure. Reaction was run using 11 (65.4 mg, $0.3 \mathrm{mmol}),\left[\mathrm{CuCF}_{2} \mathrm{CF}_{3}\right](1.0 \mathrm{~mL}, 0.90 \mathrm{mmol}$ in $\mathrm{DMF}$ ). The product was purified by flash column chromatography on silica gel (hexane/ $\mathrm{Et}_{2} \mathrm{O}$ ) and obtained a yellow oil $(0.26 \mathrm{mmol}, 85.7 \mathrm{mg}, 85 \%, E / Z=92: 8$, allylic : vinylic $>50: 1), \mathrm{R}_{\mathrm{f}}=0.6$ (hexane : 
$\left.\mathrm{Et}_{2} \mathrm{O}=2: 1\right) .{ }^{1} \mathbf{H}$ NMR $\left(400 \mathrm{MHz}, \mathrm{CDCl}_{3}\right): \delta 7.89(\mathrm{~d}, J=8.8 \mathrm{~Hz}, 2 \mathrm{H}), 6.88(\mathrm{~d}, J=8.8 \mathrm{~Hz}, 2 \mathrm{H}), 5.77-$ $5.69(\mathrm{~m}, 1 \mathrm{H}), 5.46-5.39(\mathrm{~m}, 1 \mathrm{H}), 3.99(\mathrm{t}, J=6.4 \mathrm{~Hz}, 2 \mathrm{H}), 2.73(\mathrm{td}, J=17.6 \mathrm{~Hz}, J=7.2 \mathrm{~Hz}, 2 \mathrm{H}), 2.51(\mathrm{~s}$, $3 \mathrm{H}), 2.25$ (q, $J=7.1 \mathrm{~Hz}, 2 \mathrm{H}), 1.88(\mathrm{~m}, 2 \mathrm{H}) \mathrm{ppm} .{ }^{13} \mathbf{C} \mathbf{N M R}\left(126 \mathrm{MHz}, \mathrm{CDCl}_{3}\right): \delta 196.8,163.0,137.5$, $130.6,130.3,119.2\left(\mathrm{qt}, J_{C-F}=286.3 \mathrm{~Hz}, J_{C-F}=36.2 \mathrm{~Hz}\right), 117.7\left(\mathrm{t}, J_{C-F}=4.1 \mathrm{~Hz}\right), 114.8\left(\mathrm{tq}, J_{C-F}=252.4\right.$ $\left.\mathrm{Hz}, J_{C-F}=37.3 \mathrm{~Hz}\right), 114.1,67.2,34.5\left(\mathrm{t}, J_{C-F}=22.4 \mathrm{~Hz}\right), 28.9,28.3,26.3$ ppm. ${ }^{19} \mathbf{F}$ NMR $(470 \mathrm{MHz}$, $\left.\mathrm{CDCl}_{3}\right): E$ isomer: $\delta-85.76(\mathrm{~s}, 3 \mathrm{~F}),-118.19(\mathrm{t}, J=17.4 \mathrm{~Hz}, 2 \mathrm{~F}) ; Z$ isomer: $\delta-85.98(\mathrm{~s}, 3 \mathrm{~F}),-118.02(\mathrm{t}, J$ $=17.9 \mathrm{~Hz}, 2 \mathrm{~F})$. HRMS m/z (ESI): calcd. for $\mathrm{C}_{16} \mathrm{H}_{17} \mathrm{~F}_{5} \mathrm{O}_{2} \mathrm{Na}[\mathrm{M}+\mathrm{Na}]^{+}: 359.1041$; found: 359.1039 .

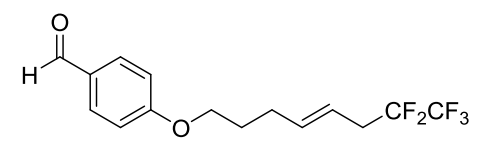

2m: (E)-4-((7,7,8,8,8-pentafluorooct-4-en-1-yl)oxy)benzaldehyde. Prepared according to the general procedure. Reaction was run using $1 \mathbf{m}(61.2 \mathrm{mg}, 0.3 \mathrm{mmol}),\left[\mathrm{CuCF}_{2} \mathrm{CF}_{3}\right]$ (1.0 mL, $0.90 \mathrm{mmol}$ in DMF). The product was purified by flash column chromatography on silica gel (hexane/Et ${ }_{2} \mathrm{O}$ ) and obtained a yellow oil $(0.25 \mathrm{mmol}, 81.1 \mathrm{mg}, 84 \%, E / Z=93: 7$, allylic : vinylic $>50: 1), \mathrm{R}_{\mathrm{f}}=0.6$ (hexane $: \mathrm{Et}_{2} \mathrm{O}=$ 2 : 1). ${ }^{1} \mathbf{H}$ NMR (400 MHz, $\left.\mathrm{CDCl}_{3}\right): \delta 9.85(\mathrm{~s}, 1 \mathrm{H}), 7.80(\mathrm{~d}, J=8.4 \mathrm{~Hz}, 2 \mathrm{H}), 6.96(\mathrm{~d}, J=8.4 \mathrm{~Hz}, 2 \mathrm{H})$, 5.77-5.70 (m, 1H), 5.47-5.40 (m, 1H), $4.02(\mathrm{t}, J=6.2 \mathrm{~Hz}, 2 \mathrm{H}), 2.74(\mathrm{td}, J=17.2 \mathrm{~Hz}, J=7.2 \mathrm{~Hz}, 2 \mathrm{H})$, $2.26(\mathrm{q}, J=7.1 \mathrm{~Hz}, 2 \mathrm{H}), 1.89$ (m, 2H) ppm. ${ }^{13} \mathbf{C ~ N M R}\left(126 \mathrm{MHz}, \mathrm{CDCl}_{3}\right): \delta 190.9,164.1,137.4,132.0$, $130.0,119.2$ (qt, $\left.J_{C-F}=286.3 \mathrm{~Hz}, J_{C-F}=36.4 \mathrm{~Hz}\right), 117.8\left(\mathrm{t}, J_{C-F}=4.3 \mathrm{~Hz}\right), 114.8\left(\mathrm{tq}, J_{C-F}=252.3 \mathrm{~Hz}, J_{C-}\right.$ $\left.{ }_{F}=37.2 \mathrm{~Hz}\right), 114.8,67.3,34.6\left(\mathrm{t}, J_{C-F}=22.4 \mathrm{~Hz}\right), 28.9,28.3 \mathrm{ppm} .{ }^{19} \mathbf{F} \mathbf{N M R}\left(470 \mathrm{MHz}, \mathrm{CDCl}_{3}\right): E$ isomer: $\delta-85.77(\mathrm{~s}, 3 \mathrm{~F}),-118.21(\mathrm{t}, J=17.4 \mathrm{~Hz}, 2 \mathrm{~F})$; $Z$ isomer: $\delta-86.00(\mathrm{~s}, 3 \mathrm{~F}),-118.04(\mathrm{t}, J=17.9 \mathrm{~Hz}, 2 \mathrm{~F})$. HRMS m/z (ESI): calcd. for $\mathrm{C}_{15} \mathrm{H}_{16} \mathrm{~F}_{5} \mathrm{O}_{2}[\mathrm{M}+\mathrm{H}]^{+}:$323.1065; found: 323.1061 .

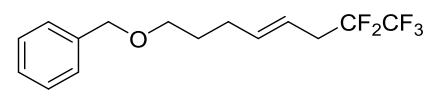

2n: (E)-(((7,7,8,8,8-pentafluorooct-4-en-1-yl)oxy)methyl)benzene. Prepared according to the general procedure. Reaction was run using $1 \mathbf{n}(57.0 \mathrm{mg}, 0.3 \mathrm{mmol}),\left[\mathrm{CuCF}_{2} \mathrm{CF}_{3}\right]$ (1.0 mL, $0.90 \mathrm{mmol}$ in DMF). The product was purified by flash column chromatography on silica gel (hexane/Et ${ }_{2} \mathrm{O}$ ) and obtained a colorless oil $(0.25 \mathrm{mmol}, 76.7 \mathrm{mg}, 83 \%, E / Z=91: 9$, allylic : vinylic $=31: 1), \mathrm{R}_{\mathrm{f}}=0.7$ (hexane $: \mathrm{Et}_{2} \mathrm{O}$ $=10: 1) .{ }^{1} \mathbf{H}$ NMR $\left(400 \mathrm{MHz}, \mathrm{CDCl}_{3}\right): \delta$ 7.38-7.34 (m, 4H), 7.33-7.28 (m, 1H), 5.76-5.69 (m, 1H), 5.46$5.38(\mathrm{~m}, 1 \mathrm{H}), 4.52(\mathrm{~s}, 2 \mathrm{H}), 3.50(\mathrm{t}, J=6.4 \mathrm{~Hz}, 2 \mathrm{H}), 2.75(\mathrm{td}, J=17.6 \mathrm{~Hz}, J=7.2 \mathrm{~Hz}, 2 \mathrm{H}), 2.20(\mathrm{q}, J=$ $7.2 \mathrm{~Hz}, 2 \mathrm{H}), 1.74(\mathrm{~m}, 2 \mathrm{H}) \mathrm{ppm} .{ }^{13} \mathbf{C}$ NMR (101 MHz, $\left.\mathrm{CDCl}_{3}\right): \delta 138.7,138.2,128.5,127.8,127.7,119.3$ (qt, $\left.J_{C-F}=286.7 \mathrm{~Hz}, J_{C-F}=36.4 \mathrm{~Hz}\right), 117.1\left(\mathrm{t}, J_{C-F}=4.4 \mathrm{~Hz}\right), 114.9\left(\mathrm{tq}, J_{C-F}=252.8 \mathrm{~Hz}, J_{C-F}=37.2 \mathrm{~Hz}\right)$, $73.1,69.5,34.7\left(\mathrm{t}, J_{C-F}=22.5 \mathrm{~Hz}\right), 29.3,29.1 \mathrm{ppm} .{ }^{19} \mathbf{F} \mathbf{N M R}\left(470 \mathrm{MHz}, \mathrm{CDCl}_{3}\right): E$ isomer: $\delta-85.77(\mathrm{~s}$, 3F), -118.24 (t, $J=17.6 \mathrm{~Hz}, 2 \mathrm{~F})$; $Z$ isomer: $\delta-85.96(\mathrm{~s}, 3 \mathrm{~F}),-118.04(\mathrm{t}, J=17.9 \mathrm{~Hz}, 2 \mathrm{~F}) . \mathbf{H R M S} \mathrm{m} / \mathrm{z}$ (APCI): calcd. for $\mathrm{C}_{15} \mathrm{H}_{17} \mathrm{~F}_{5} \mathrm{ONa}[\mathrm{M}+\mathrm{Na}]^{+}: 331.1091$; found: 331.1089 .

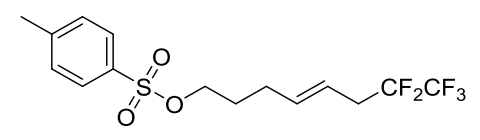

2o: (E)-7,7,8,8,8-pentafluorooct-4-en-1-yl 4-methylbenzenesulfonate. Prepared according to the general procedure. Reaction was run using $10(76.2 \mathrm{mg}, 0.3 \mathrm{mmol}),\left[\mathrm{CuCF}_{2} \mathrm{CF}_{3}\right](1.0 \mathrm{~mL}, 0.90 \mathrm{mmol}$ in $\mathrm{DMF}$ ). The product was purified by flash column chromatography on silica gel (hexane/Et ${ }_{2} \mathrm{O}$ ) and obtained a yellow oil $(0.17 \mathrm{mmol}, 62.5 \mathrm{mg}, 56 \%, E / Z=92: 8$, allylic : vinylic $>50: 1), \mathrm{R}_{\mathrm{f}}=0.5$ (hexane : 
$\left.\mathrm{Et}_{2} \mathrm{O}=2: 1\right) .{ }^{1} \mathbf{H}$ NMR $\left(400 \mathrm{MHz}, \mathrm{CDCl}_{3}\right): \delta 7.77(\mathrm{~d}, J=8.0 \mathrm{~Hz}, 2 \mathrm{H}), 7.33(\mathrm{~d}, J=8.0 \mathrm{~Hz}, 2 \mathrm{H}), 5.61$ $5.53(\mathrm{~m}, 1 \mathrm{H}), 5.34-5.27(\mathrm{~m}, 1 \mathrm{H}), 4.00(\mathrm{t}, J=6.4 \mathrm{~Hz}, 2 \mathrm{H}), 2.67(\mathrm{td}, J=17.6 \mathrm{~Hz}, J=7.2 \mathrm{~Hz}, 2 \mathrm{H}), 2.43(\mathrm{~s}$, $3 \mathrm{H}), 2.10$ (q, $J=7.2 \mathrm{~Hz}, 2 \mathrm{H}), 1.72(\mathrm{~m}, 2 \mathrm{H}) \mathrm{ppm} .{ }^{13} \mathbf{C} \mathbf{N M R}\left(101 \mathrm{MHz}, \mathrm{CDCl}_{3}\right): \delta 145.0,136.6,133.1$, $129.9,128.0,119.2\left(\mathrm{qt}, J_{C-F}=286.7 \mathrm{~Hz}, J_{C-F}=36.3 \mathrm{~Hz}\right), 118.2\left(\mathrm{t}, J_{C-F}=4.4 \mathrm{~Hz}\right), 114.7\left(\mathrm{tq}, J_{C-F}=252.8\right.$ $\left.\mathrm{Hz}, J_{C-F}=37.1 \mathrm{~Hz}\right), 67.6,34.5\left(\mathrm{t}, J_{C-F}=22.5 \mathrm{~Hz}\right), 28.3,28.1,21.6$ ppm. ${ }^{19} \mathbf{F} \mathbf{N M R}\left(470 \mathrm{MHz}, \mathrm{CDCl}_{3}\right): E$ isomer: $\delta-85.82(\mathrm{~s}, 3 \mathrm{~F}),-118.28(\mathrm{t}, J=17.6 \mathrm{~Hz}, 2 \mathrm{~F}) ; Z$ isomer: $\delta-85.93(\mathrm{~s}, 3 \mathrm{~F}),-118.00(\mathrm{t}, J=17.6 \mathrm{~Hz}$, 2F). HRMS m/z (ESI): calcd. for $\mathrm{C}_{15} \mathrm{H}_{17} \mathrm{~F}_{5} \mathrm{O}_{3} \mathrm{SNa}[\mathrm{M}+\mathrm{Na}]^{+}$: 395.0711 ; found: 395.0706 .

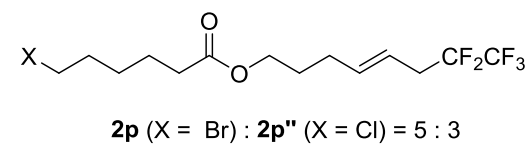

2p/2p": (E)-7,7,8,8,8-pentafluorooct-4-en-1-yl 6-bromo/-Chlorohexanoate. Prepared according to the general procedure. Reaction was run using $1 \mathbf{p}(82.8 \mathrm{mg}, 0.3 \mathrm{mmol}),\left[\mathrm{CuCF}_{2} \mathrm{CF}_{3}\right](1.0 \mathrm{~mL}, 0.90 \mathrm{mmol}$ in DMF). The product was purified by flash column chromatography on silica gel (hexane/Et ${ }_{2} \mathrm{O}$ ) and obtained an inseparable mixture of $\mathbf{2 p}$ and $\mathbf{2 p}$ " as a colorless oil $(\mathbf{2 p}: \mathbf{2} \mathbf{p} "=5: 3$, total $0.20 \mathrm{mmol}, 77.0$ $\mathrm{mg}, 68 \%, E / Z$ of $\mathbf{2 p}=93: 7, E / Z$ of $\mathbf{2 p} "=93: 7$, allylic : vinylic $>50: 1), \mathrm{R}_{\mathrm{f}}=0.5$ (hexane $: \mathrm{Et}_{2} \mathrm{O}=5$ : $1)$.

2p: ${ }^{1} \mathbf{H}$ NMR $\left(400 \mathrm{MHz}, \mathrm{CDCl}_{3}\right): \delta 5.72-5.65(\mathrm{~m}, 1 \mathrm{H}), 5.44-5.37(\mathrm{~m}, 1 \mathrm{H}), 4.05(\mathrm{t}, J=6.6 \mathrm{~Hz}, 2 \mathrm{H}), 3.38$ $(\mathrm{t}, J=6.8 \mathrm{~Hz}, 2 \mathrm{H}), 2.72(\mathrm{td}, J=17.6 \mathrm{~Hz}, J=7.2 \mathrm{~Hz}, 2 \mathrm{H}), 2.30(\mathrm{t}, J=7.4 \mathrm{~Hz}, 2 \mathrm{H}), 2.12(\mathrm{q}, J=7.2 \mathrm{~Hz}$, $2 \mathrm{H}), 1.85(\mathrm{~m}, 2 \mathrm{H}), 1.71(\mathrm{~m}, 2 \mathrm{H}), 1.63(\mathrm{~m}, 2 \mathrm{H}), 1.46(\mathrm{~m}, 2 \mathrm{H}) \mathrm{ppm} .{ }^{13} \mathbf{C} \mathbf{N M R}\left(126 \mathrm{MHz}, \mathrm{CDCl}_{3}\right): \delta 173.5$, $137.4,119.2\left(\mathrm{qt}, J_{C-F}=286.1 \mathrm{~Hz}, J_{C-F}=36.4 \mathrm{~Hz}\right), 117.6\left(\mathrm{t}, J_{C-F}=4.3 \mathrm{~Hz}\right), 114.8\left(\mathrm{tq}, J_{C-F}=252.3 \mathrm{~Hz}, J_{C-}\right.$ $\left.{ }_{F}=37.2 \mathrm{~Hz}\right), 63.6,34.6\left(\mathrm{t}, J_{C-F}=22.5 \mathrm{~Hz}\right), 34.1,33.5,32.5,29.0,28.0,27.7,24.2 \mathrm{ppm} .{ }^{19} \mathbf{F}$ NMR $(470$ $\mathrm{MHz}, \mathrm{CDCl}_{3}$ ): $E$ isomer: $\delta-85.78(\mathrm{~s}, 3 \mathrm{~F}),-118.25(\mathrm{t}, J=17.6 \mathrm{~Hz}, 2 \mathrm{~F}) ; Z$ isomer: $\delta-85.96(\mathrm{~s}, 3 \mathrm{~F}),-118.03$ (t, $J=17.9 \mathrm{~Hz}, 2 \mathrm{~F})$. HRMS m/z (ESI): calcd. for $\mathrm{C}_{14} \mathrm{H}_{20} \mathrm{BrF}_{5} \mathrm{O}_{2} \mathrm{Na}[\mathrm{M}+\mathrm{Na}]^{+}$: 417.0459 ; found: 417.0454 .

2p": ${ }^{1} \mathbf{H}$ NMR (400 MHz, $\left.\mathrm{CDCl}_{3}\right): \delta 5.72-5.65(\mathrm{~m}, 1 \mathrm{H}), 5.44-5.37(\mathrm{~m}, 1 \mathrm{H}), 4.05(\mathrm{t}, J=6.6 \mathrm{~Hz}, 2 \mathrm{H}), 3.51$ (t, $J=6.6 \mathrm{~Hz}, 2 \mathrm{H}), 2.72(\mathrm{td}, J=17.6 \mathrm{~Hz}, J=7.2 \mathrm{~Hz}, 2 \mathrm{H}), 2.30(\mathrm{t}, J=7.4 \mathrm{~Hz}, 2 \mathrm{H}), 2.12(\mathrm{q}, J=7.2 \mathrm{~Hz}$, 2H), $1.77(\mathrm{~m}, 2 \mathrm{H}), 1.71(\mathrm{~m}, 2 \mathrm{H}), 1.63(\mathrm{~m}, 2 \mathrm{H}), 1.45(\mathrm{~m}, 2 \mathrm{H}) \mathrm{ppm} .{ }^{13} \mathbf{C} \mathbf{N M R}\left(126 \mathrm{MHz}, \mathrm{CDCl}_{3}\right): \delta 173.5$, $137.4,119.2\left(\mathrm{qt}, J_{C-F}=286.1 \mathrm{~Hz}, J_{C-F}=36.4 \mathrm{~Hz}\right), 117.6\left(\mathrm{t}, J_{C-F}=4.3 \mathrm{~Hz}\right), 114.8\left(\mathrm{tq}, J_{C-F}=252.3 \mathrm{~Hz}, J_{C-}\right.$ $\left.{ }_{F}=37.2 \mathrm{~Hz}\right), 63.6,44.8,34.6\left(\mathrm{t}, J_{C-F}=22.5 \mathrm{~Hz}\right), 34.1,32.3,29.0,28.0,26.5,24.3 \mathrm{ppm} .{ }^{19} \mathbf{F} \mathbf{N M R}(470$ $\mathrm{MHz}, \mathrm{CDCl}_{3}$ ): $E$ isomer: $\delta-85.78(\mathrm{~s}, 3 \mathrm{~F}),-118.25(\mathrm{t}, J=17.6 \mathrm{~Hz}, 2 \mathrm{~F}) ; Z$ isomer: $\delta-85.96(\mathrm{~s}, 3 \mathrm{~F}),-118.03$ (t, $J=17.9 \mathrm{~Hz}, 2 \mathrm{~F})$. HRMS m/z (ESI): calcd. for $\mathrm{C}_{14} \mathrm{H}_{20} \mathrm{ClF}_{5} \mathrm{O}_{2} \mathrm{Na}[\mathrm{M}+\mathrm{Na}]^{+}$: 373.0964 ; found: 373.0960 .<smiles>COC(=O)CCCCCCC/C=C/CC(F)(F)C(F)(F)F</smiles>

2q: methyl (E)-12,12,13,13,13-pentafluorotridec-9-enoate. Prepared according to the general procedure. Reaction was run using 1q $(59.4 \mathrm{mg}, 0.3 \mathrm{mmol}),\left[\mathrm{CuCF}_{2} \mathrm{CF}_{3}\right]$ (1.0 mL, $0.90 \mathrm{mmol}$ in DMF). The product was purified by flash column chromatography on silica gel (hexane/Et ${ }_{2} \mathrm{O}$ ) and obtained a colorless oil $(0.24 \mathrm{mmol}, 76.8 \mathrm{mg}, 81 \%, E / Z=94: 6$, allylic : vinylic $=33: 1), \mathrm{R}_{\mathrm{f}}=0.7$ (hexane $: \mathrm{Et}_{2} \mathrm{O}$ $=5: 1) .{ }^{1} \mathbf{H}$ NMR $\left(400 \mathrm{MHz}, \mathrm{CDCl}_{3}\right): \delta 5.70-5.62(\mathrm{~m}, 1 \mathrm{H}), 5.38-5.30(\mathrm{~m}, 1 \mathrm{H}), 3.63(\mathrm{~s}, 3 \mathrm{H}), 2.70(\mathrm{td}, J$ $=17.6 \mathrm{~Hz}, J=7.2 \mathrm{~Hz}, 2 \mathrm{H}), 2.27(\mathrm{t}, J=7.6 \mathrm{~Hz}, 2 \mathrm{H}), 2.02(\mathrm{q}, J=6.8 \mathrm{~Hz}, 2 \mathrm{H}), 1.59(\mathrm{~m}, 2 \mathrm{H}), 1.34(\mathrm{~m}, 2 \mathrm{H})$, 1.31-1.23 (m, 6H) ppm. ${ }^{13} \mathbf{C}$ NMR $\left(101 \mathrm{MHz}, \mathrm{CDCl}_{3}\right): \delta 174.4,138.9,119.3\left(\mathrm{qt}, J_{C-F}=286.7 \mathrm{~Hz}, J_{C-F}=\right.$ $36.5 \mathrm{~Hz}), 116.5\left(\mathrm{t}, J_{C-F}=4.3 \mathrm{~Hz}\right), 114.9\left(\mathrm{tq}, J_{C-F}=252.6 \mathrm{~Hz}, J_{C-F}=37.1 \mathrm{~Hz}\right), 51.4,34.7\left(\mathrm{t}, J_{C-F}=22.5\right.$ $\mathrm{Hz}$ ), 34.1, 32.6, 29.2, 29.1, 28.9, $25.0 \mathrm{ppm}$ (one carbon missing due to overlap). ${ }^{19} \mathbf{F}$ NMR (470 MHz, 
$\left.\mathrm{CDCl}_{3}\right): E$ isomer: $\delta-85.76(\mathrm{~s}, 3 \mathrm{~F}),-118.26(\mathrm{t}, J=17.6 \mathrm{~Hz}, 2 \mathrm{~F}) ; Z$ isomer: $\delta-85.94(\mathrm{~s}, 3 \mathrm{~F}),-118.00(\mathrm{t}, J$ $=17.6 \mathrm{~Hz}, 2 \mathrm{~F})$. HRMS m/z (ESI): calcd. for $\mathrm{C}_{14} \mathrm{H}_{21} \mathrm{~F}_{5} \mathrm{O}_{2} \mathrm{Na}[\mathrm{M}+\mathrm{Na}]^{+}:$339.1354; found: 339.1351 .<smiles>O=C(CCCCCCCC=CCC(F)(F)F)N1CCOCC1</smiles>

2r: (E)-12,12,13,13,13-pentafluoro-1-morpholinotridec-9-en-1-one. Prepared according to the general procedure. Reaction was run using $1 \mathbf{r}(75.9 \mathrm{mg}, 0.3 \mathrm{mmol}),\left[\mathrm{CuCF}_{2} \mathrm{CF}_{3}\right](1.0 \mathrm{~mL}, 0.90 \mathrm{mmol}$ in $\mathrm{DMF})$. The product was purified by flash column chromatography on silica gel (hexane $\left./ \mathrm{Et}_{2} \mathrm{O}\right)$ and obtained a yellow oil $(0.25 \mathrm{mmol}, 91.3 \mathrm{mg}, 82 \%, E / Z=93: 7$, allylic : vinylic $=28: 1), \mathrm{R}_{\mathrm{f}}=0.3$ (hexane : EtOAc $=3: 2) .{ }^{1} \mathbf{H}$ NMR $\left(400 \mathrm{MHz}, \mathrm{CDCl}_{3}\right): \delta 5.68-5.61(\mathrm{~m}, 1 \mathrm{H}), 5.36-5.26(\mathrm{~m}, 1 \mathrm{H}), 3.62(\mathrm{t}, J=4.8$ $\mathrm{Hz}, 4 \mathrm{H}), 3.57$ (t, $J=4.4 \mathrm{~Hz}, 2 \mathrm{H}), 3.42(\mathrm{t}, J=4.4 \mathrm{~Hz}, 2 \mathrm{H}), 2.69(\mathrm{td}, J=17.6 \mathrm{~Hz}, J=7.2 \mathrm{~Hz}, 2 \mathrm{H}), 2.26$ (t, $J=7.6 \mathrm{~Hz}, 2 \mathrm{H}), 2.01(\mathrm{q}, J=6.8 \mathrm{~Hz}, 2 \mathrm{H}), 1.58(\mathrm{~m}, 2 \mathrm{H}), 1.33(\mathrm{~m}, 2 \mathrm{H}), 1.29-1.22(\mathrm{~m}, 6 \mathrm{H}) \mathrm{ppm} .{ }^{13} \mathbf{C} \mathbf{N M R}$ $\left(126 \mathrm{MHz}_{\mathrm{CDCl}}\right): \delta 171.9,138.9,119.2\left(\mathrm{qt}, J_{C-F}=286.3 \mathrm{~Hz}, J_{C-F}=36.4 \mathrm{~Hz}\right), 116.4\left(\mathrm{t}, J_{C-F}=4.2 \mathrm{~Hz}\right)$, $114.9\left(\mathrm{tq}, J_{C-F}=252.1 \mathrm{~Hz}, J_{C-F}=37.0 \mathrm{~Hz}\right), 67.0,66.7,46.1,41.9,34.6\left(\mathrm{t}, J_{C-F}=22.4 \mathrm{~Hz}\right), 33.1,32.5$, 29.4, 29.2, 28.9, 28.8, 25.2 ppm. ${ }^{19} \mathbf{F}$ NMR (470 MHz, $\left.\mathrm{CDCl}_{3}\right): E$ isomer: $\delta-85.77(\mathrm{~s}, 3 \mathrm{~F}),-118.22(\mathrm{t}, J$ $=17.6 \mathrm{~Hz}, 2 \mathrm{~F}) ; Z$ isomer: $\delta-85.95(\mathrm{~s}, 3 \mathrm{~F}),-118.02(\mathrm{t}, J=17.9 \mathrm{~Hz}, 2 \mathrm{~F}) . \mathbf{H R M S ~ m} / \mathrm{z}(\mathrm{ESI})$ : calcd. for $\mathrm{C}_{17} \mathrm{H}_{27} \mathrm{~F}_{5} \mathrm{NO}_{2}[\mathrm{M}+\mathrm{H}]^{+}: 372.1957$; found: 372.1953 .

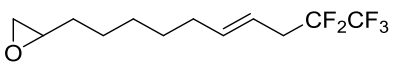

2s: (E)-2-(9,9,10,10,10-pentafluorodec-6-en-1-yl)oxirane. Prepared according to the general procedure. Reaction was run using $1 \mathrm{~s}$ (46.2 mg, $0.3 \mathrm{mmol}),\left[\mathrm{CuCF}_{2} \mathrm{CF}_{3}\right](1.0 \mathrm{~mL}, 0.90 \mathrm{mmol}$ in DMF). The product was purified by flash column chromatography on silica gel (hexane/Et ${ }_{2} \mathrm{O}$ ) and obtained a colorless oil (0.22 mmol, $60.4 \mathrm{mg}, 74 \%, E / Z=92: 8$, allylic : vinylic $=45: 1), \mathrm{R}_{\mathrm{f}}=0.7$ (hexane : $\left.\mathrm{Et}_{2} \mathrm{O}=5: 2\right) .{ }^{1} \mathbf{H}$ NMR (400 MHz, $\left.\mathrm{CDCl}_{3}\right): \delta 5.71-5.64(\mathrm{~m}, 1 \mathrm{H}), 5.39-5.32(\mathrm{~m}, 1 \mathrm{H}), 2.90-2.85(\mathrm{~m}, 1 \mathrm{H}), 2.71(\mathrm{td}, J=17.8$ $\mathrm{Hz}, J=6.8 \mathrm{~Hz}, 2 \mathrm{H}), 2.74-2.69(\mathrm{~m}, 1 \mathrm{H}), 2.43(\mathrm{dd}, J=4.8 \mathrm{~Hz}, J=2.8 \mathrm{~Hz}, 1 \mathrm{H}), 2.05(\mathrm{q}, J=6.7 \mathrm{~Hz}, 2 \mathrm{H})$, 1.54-1.30 (m, 8H) ppm. ${ }^{13} \mathbf{C}$ NMR (101 MHz, $\left.\mathrm{CDCl}_{3}\right): \delta 138.8,119.3$ (qt, $J_{C-F}=286.6 \mathrm{~Hz}, J_{C-F}=36.4$ $\mathrm{Hz}), 116.7\left(\mathrm{t}, J_{C-F}=4.3 \mathrm{~Hz}\right), 114.9\left(\mathrm{tq}, J_{C-F}=252.6 \mathrm{~Hz}, J_{C-F}=37.2 \mathrm{~Hz}\right), 52.4,47.1,34.7\left(\mathrm{t}, J_{C-F}=22.5\right.$ $\mathrm{Hz}$ ), 32.5, 32.5, 28.9, $25.9 \mathrm{ppm}$ (one carbon missing due to overlap). ${ }^{19} \mathbf{F} \mathbf{N M R}\left(470 \mathrm{MHz}, \mathrm{CDCl}_{3}\right): E$ isomer: $\delta-85.76(\mathrm{~s}, 3 \mathrm{~F}),-118.26(\mathrm{t}, J=17.6 \mathrm{~Hz}, 2 \mathrm{~F})$; $Z$ isomer: $\delta-85.94(\mathrm{~s}, 3 \mathrm{~F}),-118.00(\mathrm{t}, J=17.6 \mathrm{~Hz}$, 2F). HRMS m/z (APCI): calcd. for $\mathrm{C}_{12} \mathrm{H}_{18} \mathrm{~F}_{5} \mathrm{O}[\mathrm{M}+\mathrm{H}]^{+}: 273.1272$; found: 273.1271.

$\mathrm{CF}_{2} \mathrm{CF}_{3}$

2t: (E)-12,12,13,13,13-pentafluorotridec-9-en-1-ol. Prepared according to the general procedure. Reaction was run using $1 \mathrm{t}(51.0 \mathrm{mg}, 0.3 \mathrm{mmol}),\left[\mathrm{CuCF}_{2} \mathrm{CF}_{3}\right](1.0 \mathrm{~mL}, 0.90 \mathrm{mmol}$ in $\mathrm{DMF})$. The product was purified by flash column chromatography on silica gel (hexane/Et ${ }_{2} \mathrm{O}$ ) and obtained a colorless oil (0.24 mmol, $70.0 \mathrm{mg}, 81 \%, E / Z=92: 8$, allylic : vinylic $=48: 1), \mathrm{R}_{\mathrm{f}}=0.3$ (hexane : EtOAc = $\left.3: 1\right) .{ }^{1} \mathbf{H}$ NMR (400 MHz, $\left.\mathrm{CDCl}_{3}\right): \delta 5.71-5.64(\mathrm{~m}, 1 \mathrm{H}), 5.38-5.31(\mathrm{~m}, 1 \mathrm{H}), 3.60(\mathrm{t}, J=6.6 \mathrm{~Hz}, 2 \mathrm{H}), 2.71(\mathrm{td}, J=$ $17.6 \mathrm{~Hz}, J=7.2 \mathrm{~Hz}, 2 \mathrm{H}), 2.04(\mathrm{~s}, 1 \mathrm{H}), 2.03$ (q, $J=6.8 \mathrm{~Hz}, 2 \mathrm{H}), 1.53(\mathrm{~m}, 2 \mathrm{H}), 1.34(\mathrm{~m}, 2 \mathrm{H}), 1.32-1.22$ $(\mathrm{m}, 8 \mathrm{H}) \mathrm{ppm} .{ }^{13} \mathbf{C} \mathbf{N M R}\left(126 \mathrm{MHz}, \mathrm{CDCl}_{3}\right): \delta 139.0,119.3\left(\mathrm{qt}, J_{C-F}=286.1 \mathrm{~Hz}, J_{C-F}=36.4 \mathrm{~Hz}\right), 116.5$ $\left(\mathrm{t}, J_{C-F}=4.3 \mathrm{~Hz}\right), 114.9\left(\mathrm{tq}, J_{C-F}=252.6 \mathrm{~Hz}, J_{C-F}=37.0 \mathrm{~Hz}\right), 63.0,34.7\left(\mathrm{t}, J_{C-F}=22.4 \mathrm{~Hz}\right), 32.8,32.6$, 29.5, 29.5, 29.1, 29.0, 25.8 ppm. ${ }^{19} \mathbf{F}$ NMR (470 MHz, $\left.\mathrm{CDCl}_{3}\right): E$ isomer: $\delta-85.76(\mathrm{~s}, 3 \mathrm{~F}),-118.26(\mathrm{t}, J$ $=17.4 \mathrm{~Hz}, 2 \mathrm{~F})$; $Z$ isomer: $\delta-85.95(\mathrm{~s}, 3 \mathrm{~F}),-117.99(\mathrm{t}, J=17.6 \mathrm{~Hz}, 2 \mathrm{~F})$. HRMS m/z (APCI): calcd. for 
$\mathrm{C}_{13} \mathrm{H}_{22} \mathrm{~F}_{5} \mathrm{O}[\mathrm{M}+\mathrm{H}]^{+}:$289.1585; found: 289.1583 .<smiles>O=C(O)CCCCCCCC=CCCC(F)(F)F</smiles>

2u: (E)-12,12,13,13,13-pentafluorotridec-9-enoic acid. Prepared according to the general procedure. Reaction was run using $1 \mathbf{u}(55.2 \mathrm{mg}, 0.3 \mathrm{mmol}),\left[\mathrm{CuCF}_{2} \mathrm{CF}_{3}\right]$ (1.0 mL, $0.90 \mathrm{mmol}$ in DMF). The product was purified by flash column chromatography on silica gel (hexane/ $\left.\mathrm{Et}_{2} \mathrm{O}\right)$ and obtained a yelow oil $(0.24$ mmol, $72.5 \mathrm{mg}, 80 \%, E / Z=97: 3$, allylic : vinyl $=13: 1), \mathrm{R}_{\mathrm{f}}=0.2$ (hexane $:$ EtOAc $\left.=2: 1\right) .{ }^{1} \mathbf{H}$ NMR $\left(400 \mathrm{MHz}, \mathrm{CDCl}_{3}\right): \delta 10.56(\mathrm{~s}, 1 \mathrm{H}), 5.73-5.65(\mathrm{~m}, 1 \mathrm{H}), 5.40-5.33(\mathrm{~m}, 1 \mathrm{H}), 2.73$ (td, $J=17.6 \mathrm{~Hz}, J=7.2$ $\mathrm{Hz}, 2 \mathrm{H}), 2.34$ (t, $J=7.4 \mathrm{~Hz}, 2 \mathrm{H}), 2.05(\mathrm{q}, J=6.9 \mathrm{~Hz}, 2 \mathrm{H}), 1.63(\mathrm{~m}, 2 \mathrm{H}), 1.35(\mathrm{~m}, 2 \mathrm{H}), 1.33-1.24(\mathrm{~m}, 6 \mathrm{H})$ ppm. ${ }^{13} \mathrm{C}$ NMR (126 MHz, $\left.\mathrm{CDCl}_{3}\right): \delta 180.7,139.0,119.3\left(\mathrm{qt}, J_{C-F}=286.1 \mathrm{~Hz}, J_{C-F}=36.4 \mathrm{~Hz}\right), 116.6(\mathrm{t}$, $J_{C-F}=4.3 \mathrm{~Hz}$ ), $115.0\left(\mathrm{tq}, J_{C-F}=252.3 \mathrm{~Hz}, J_{C-F}=37.0 \mathrm{~Hz}\right.$ ), $34.7\left(\mathrm{t}, J_{C-F}=22.4 \mathrm{~Hz}\right.$ ), 34.2, 32.6, 29.2, 29.2, 28.9, 28.9, 24.8 ppm. ${ }^{19} \mathbf{F}$ NMR (470 MHz, $\left.\mathrm{CDCl}_{3}\right): E$ isomer: $\delta-85.77(\mathrm{~s}, 3 \mathrm{~F}),-118.28(\mathrm{t}, J=17.6 \mathrm{~Hz}$, 2F); $Z$ isomer: $\delta-85.97$ (s, 3F), -118.03 (t, $J=17.9 \mathrm{~Hz}, 2 \mathrm{~F}$ ). HRMS m/z (APCI): calcd. for $\mathrm{C}_{13} \mathrm{H}_{18} \mathrm{~F}_{5} \mathrm{O}_{2}$ [M-H]': 301.1232; found: 301.1232 .

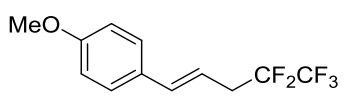

2v: (E)-1-methoxy-4-(4,4,5,5,5-pentafluoropent-1-en-1-yl)benzene. Prepared according to the general procedure. Reaction was run using $1 \mathbf{v}(44.4 \mathrm{mg}, 0.3 \mathrm{mmol}),\left[\mathrm{CuCF}_{2} \mathrm{CF}_{3}\right](1.0 \mathrm{~mL}, 0.90 \mathrm{mmol}$ in DMF). The product was purified by flash column chromatography on silica gel (hexane/Et ${ }_{2} \mathrm{O}$ ) and obtained a colorless oil $\left(0.17 \mathrm{mmol}, 44.7 \mathrm{mg}, 56 \%, E / Z=98: 2\right.$, allylic : vinylic $>50: 1$ ), $\mathrm{R}_{\mathrm{f}}=0.5$ (hexane : $\mathrm{Et}_{2} \mathrm{O}$ $=10: 1) .{ }^{1} \mathbf{H}$ NMR $\left(500 \mathrm{MHz}, \mathrm{CDCl}_{3}\right): \delta 7.34(\mathrm{~d}, J=8.5 \mathrm{~Hz}, 2 \mathrm{H}), 6.88(\mathrm{~d}, J=8.4 \mathrm{~Hz}, 2 \mathrm{H}), 6.56(\mathrm{~d}, J=$ $16.0 \mathrm{~Hz}, 1 \mathrm{H}), 6.02-5.96(\mathrm{~m}, 1 \mathrm{H}), 3.82(\mathrm{~s}, 3 \mathrm{H}), 2.95(\mathrm{td}, J=17.5 \mathrm{~Hz}, J=7.5 \mathrm{~Hz}, 2 \mathrm{H}) \mathrm{ppm} .{ }^{13} \mathbf{C}$ NMR $\left(126 \mathrm{MHz}, \mathrm{CDCl}_{3}\right): \delta 159.7,136.6,129.2,127.8,119.3\left(\mathrm{qt}, J_{C-F}=286.1 \mathrm{~Hz}, J_{C-F}=36.2 \mathrm{~Hz}\right), 114.9$ (tq, $\left.J_{C-F}=252.4 \mathrm{~Hz}, J_{C-F}=37.2 \mathrm{~Hz}\right), 114.2,113.9\left(\mathrm{t}, J_{C-F}=4.4 \mathrm{~Hz}\right), 55.4,35.0\left(\mathrm{t}, J_{C-F}=22.5 \mathrm{~Hz}\right) \mathrm{ppm} .{ }^{19} \mathbf{F}$ NMR (470 MHz, $\mathrm{CDCl}_{3}$ ): $E$ isomer: $\delta-85.75$ (s, 3F), -117.96 (t, $\left.J=17.4 \mathrm{~Hz}, 2 \mathrm{~F}\right)$. HRMS m/z (APCI): calcd. for $\mathrm{C}_{12} \mathrm{H}_{12} \mathrm{~F}_{5} \mathrm{O}[\mathrm{M}+\mathrm{H}]^{+}: 267.0802$; found: 267.0806 .

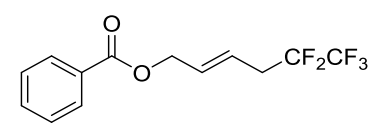

2w: (E)-5,5,6,6,6-pentafluorohex-2-en-1-yl benzoate. Prepared according to the general procedure. Reaction was run using $1 \mathbf{w}(52.8 \mathrm{mg}, 0.3 \mathrm{mmol})$, $\left[\mathrm{CuCF}_{2} \mathrm{CF}_{3}\right](1.0 \mathrm{~mL}, 0.90 \mathrm{mmol}$ in DMF). The product was purified by flash column chromatography on silica gel (hexane/Et $\mathrm{t}_{2} \mathrm{O}$ ) and obtained a colorless oil $\left(0.18 \mathrm{mmol}, 52.9 \mathrm{mg}, 60 \%, E / Z=98: 2\right.$, allylic $:$ vinylic $=7: 1$ by crude ${ }^{1} \mathrm{H} \mathrm{NMR}$, vinylic isomer was separated by column chromatography), $\mathrm{R}_{\mathrm{f}}=0.7$ (hexane : $\mathrm{Et}_{2} \mathrm{O}=5: 1$ ). ${ }^{1} \mathbf{H} \mathbf{N M R}\left(400 \mathrm{MHz}, \mathrm{CDCl}_{3}\right): \delta$ 8.06 (d, $J=7.2 \mathrm{~Hz}, 2 \mathrm{H}), 7.57$ (t, $J=7.4 \mathrm{~Hz}, 1 \mathrm{H}), 7.45$ (t, $J=7.4 \mathrm{~Hz}, 2 \mathrm{H}), 6.01-5.95$ (m, 1H), 5.86-5.79 (m, 1H), $4.84(\mathrm{~d}, J=6.0 \mathrm{~Hz}, 2 \mathrm{H}), 2.85(\mathrm{td}, J=17.6 \mathrm{~Hz}, J=6.8 \mathrm{~Hz}, 2 \mathrm{H}) \mathrm{ppm} .{ }^{13} \mathbf{C}$ NMR (101 MHz, $\left.\mathrm{CDCl}_{3}\right): \delta 166.3,133.2,132.2,130.1,129.8,128.5,121.4\left(\mathrm{t}, J_{C-F}=4.4 \mathrm{~Hz}\right), 119.2\left(\mathrm{qt}, J_{C-F}=286.6 \mathrm{~Hz}\right.$, $\left.J_{C-F}=36.1 \mathrm{~Hz}\right), 114.7\left(\mathrm{tq}, J_{C-F}=253.6 \mathrm{~Hz}, J_{C-F}=37.7 \mathrm{~Hz}\right), 64.4,34.4\left(\mathrm{t}, J_{C-F}=22.6 \mathrm{~Hz}\right)$ ppm. ${ }^{19} \mathbf{F} \mathbf{~ N M R}$ $\left(376 \mathrm{MHz}, \mathrm{CDCl}_{3}\right.$ ): $E$ isomer: $\delta-85.87$ (s, 3F), -118.05 (t, $\left.J=17.3 \mathrm{~Hz}, 2 \mathrm{~F}\right)$. HRMS m/z (ESI): calcd. for $\mathrm{C}_{13} \mathrm{H}_{11} \mathrm{~F}_{5} \mathrm{O}_{2} \mathrm{Na}[\mathrm{M}+\mathrm{Na}]^{+}: 317.0571$; found: 317.0570 . 


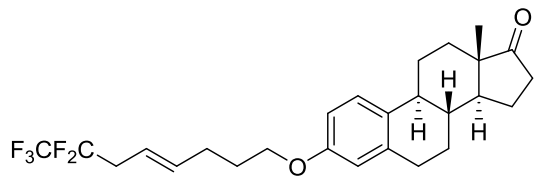

2x: (8R,9S,13S,14S)-13-methyl-3-((E)-6-((1,2,2,2-tetrafluoro-2l5-vinyl)-l2-fluoranyl)hex-4-en-1yl)oxy)-6,7,8,9,11,12,13,14,15,16-decahydro-17H-cyclopenta[a]phenanthren-17-one. Prepared according to the general procedure. Reaction was run using $\mathbf{1 x}(105.6 \mathrm{mg}, 0.3 \mathrm{mmol}),\left[\mathrm{CuCF}_{2} \mathrm{CF}_{3}\right](1.0$ $\mathrm{mL}, 0.90 \mathrm{mmol}$ in DMF). The product was purified by flash column chromatography on silica gel (hexane/ $\left.\mathrm{Et}_{2} \mathrm{O}\right)$ and obtained a white solid, melting point: $98.7-99.4{ }^{\circ} \mathrm{C}(0.28 \mathrm{mmol}, 131.1 \mathrm{mg}, 93 \%, E / Z$ $=95: 5$, allylic : vinylic $=30: 1), \mathrm{R}_{\mathrm{f}}=0.4$ (hexane $\left.: \mathrm{Et}_{2} \mathrm{O}=3: 1\right) .{ }^{1} \mathbf{H} \mathbf{~ N M R}\left(400 \mathrm{MHz}, \mathrm{CDCl}_{3}\right): \delta 7.22$ $(\mathrm{d}, J=8.8 \mathrm{~Hz}, 1 \mathrm{H}), 6.74(\mathrm{dd}, J=8.4 \mathrm{~Hz}, J=2.4 \mathrm{~Hz}, 1 \mathrm{H}), 6.67(\mathrm{~d}, J=2.4 \mathrm{~Hz}, 1 \mathrm{H}), 5.83-5.76(\mathrm{~m}, 1 \mathrm{H})$, 5.52-5.46 (m, 1H), $3.96(\mathrm{~d}, J=6.4 \mathrm{~Hz}, 2 \mathrm{H}), 2.97-2.87(\mathrm{~m}, 2 \mathrm{H}), 2.79(\mathrm{td}, J=17.6 \mathrm{~Hz}, J=6.8 \mathrm{~Hz}, 2 \mathrm{H})$, $2.79(\mathrm{dd}, J=18.8 \mathrm{~Hz}, J=8.8 \mathrm{~Hz}, 2 \mathrm{H}), 2.45-2.39$ (m, 1H), 2.29 (q, $J=7.2 \mathrm{~Hz}, 2 \mathrm{H}), 2.22-1.95$ (m, 4H), 1.89 (m, 2H), 1.70-1.42 (m, 6H), 0.94 (s, 3H) ppm. ${ }^{13} \mathbf{C}$ NMR (126 MHz, $\left.\mathrm{CDCl}_{3}\right): \delta 220.8,157.0,137.8$, $137.7,132.0,126.3,119.2\left(\mathrm{qt}, J_{C-F}=286.3 \mathrm{~Hz}, J_{C-F}=36.4 \mathrm{~Hz}\right), 117.3\left(\mathrm{t}, J_{C-F}=4.3 \mathrm{~Hz}\right), 114.8\left(\mathrm{tq}, J_{C-F}=\right.$ $\left.253.6 \mathrm{~Hz}, J_{C-F}=37.2 \mathrm{~Hz}\right), 114.5,112.1,66.8,50.4,48.0,44.0,38.4,35.8,34.5\left(\mathrm{t}, J_{C-F}=22.4 \mathrm{~Hz}\right), 31.6$, 29.7, 29.0, 28.6, 26.6, 25.9, 21.6, 13.8 ppm. ${ }^{19} \mathbf{F ~ N M R ~}\left(470 \mathrm{MHz}, \mathrm{CDCl}_{3}\right): E$ isomer: $\delta-85.76(\mathrm{~s}, 3 \mathrm{~F})$, $118.21(\mathrm{t}, J=17.6 \mathrm{~Hz}, 2 \mathrm{~F}) ; Z$ isomer: $\delta-85.97(\mathrm{~s}, 3 \mathrm{~F}),-118.01(\mathrm{t}, J=17.9 \mathrm{~Hz}, 2 \mathrm{~F}) . \mathbf{H R M S ~ m} / \mathrm{z}$ (ESI): calcd. for $\mathrm{C}_{26} \mathrm{H}_{31} \mathrm{~F}_{5} \mathrm{O}_{2}[\mathrm{M}]^{+}$: 470.2239 ; found: 470.2231 .

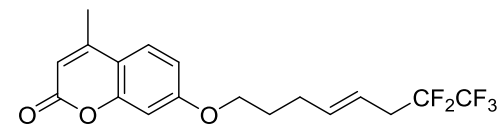

2y: (E)-4-methyl-7-((7,7,8,8,8-pentafluorooct-4-en-1-yl)oxy)-2H-chromen-2-one. Prepared according to the general procedure. Reaction was run using $1 \mathbf{y}(77.4 \mathrm{mg}, 0.3 \mathrm{mmol}),\left[\mathrm{CuCF}_{2} \mathrm{CF}_{3}\right](1.0$ $\mathrm{mL}, 0.90 \mathrm{mmol}$ in DMF). The product was purified by flash column chromatography on silica gel (hexane/ $\left.\mathrm{Et}_{2} \mathrm{O}\right)$ and obtained a yellow solid, melting point: $46.1-46.5{ }^{\circ} \mathrm{C}(0.22 \mathrm{mmol}, 81.2 \mathrm{mg}, 72 \%, E / Z$ $=96: 4$, allylic : vinylic $>50: 1), \mathrm{R}_{\mathrm{f}}=0.3$ (hexane $\left.: \mathrm{Et}_{2} \mathrm{O}=2: 1\right) .{ }^{1} \mathbf{H} \mathbf{~ N M R}\left(400 \mathrm{MHz}, \mathrm{CDCl}_{3}\right): \delta 7.44$ $(\mathrm{d}, J=8.8 \mathrm{~Hz}, 1 \mathrm{H}), 6.80(\mathrm{dd}, J=8.8 \mathrm{~Hz}, J=2.0 \mathrm{~Hz}, 1 \mathrm{H}), 6.72(\mathrm{t}, J=2.0 \mathrm{~Hz}, 1 \mathrm{H}), 6.06(\mathrm{~s}, 1 \mathrm{H}), 5.76-$ $5.69(\mathrm{~m}, 1 \mathrm{H}), 5.46-5.38(\mathrm{~m}, 1 \mathrm{H}), 3.97(\mathrm{t}, J=6.4 \mathrm{~Hz}, 2 \mathrm{H}), 2.73(\mathrm{td}, J=17.6 \mathrm{~Hz}, J=6.8 \mathrm{~Hz}, 2 \mathrm{H}), 2.34(\mathrm{~s}$, $3 \mathrm{H}), 2.25$ (q, $J=7.1 \mathrm{~Hz}, 2 \mathrm{H}), 1.88(\mathrm{~m}, 2 \mathrm{H}) \mathrm{ppm} .{ }^{13} \mathbf{C}$ NMR (101 MHz, $\left.\mathrm{CDCl}_{3}\right): \delta 162.1,161.3,155.3$, $152.7,137.4,125.6,119.2\left(\mathrm{qt}, J_{C-F}=286.7 \mathrm{~Hz}, J_{C-F}=36.4 \mathrm{~Hz}\right), 117.7\left(\mathrm{t}, J_{C-F}=4.4 \mathrm{~Hz}\right), 114.8\left(\mathrm{tq}, J_{C-F}=\right.$ $\left.252.7 \mathrm{~Hz}, J_{C-F}=37.2 \mathrm{~Hz}\right), 113.5,112.5,111.8,101.4,67.5,34.5\left(\mathrm{t}, J_{C-F}=22.5 \mathrm{~Hz}\right), 28.9,28.2,18.6 \mathrm{ppm}$. ${ }^{19}$ F NMR (376 MHz, $\mathrm{CDCl}_{3}$ ): $E$ isomer: $\delta-85.76(\mathrm{~s}, 3 \mathrm{~F}),-118.22(\mathrm{t}, J=17.5 \mathrm{~Hz}, 2 \mathrm{~F}) ; Z$ isomer: $\delta-85.97$ (s, 3F), -118.04 (t, $J=17.7 \mathrm{~Hz}, 2 \mathrm{~F})$. HRMS m/z (ESI): calcd. for $\mathrm{C}_{18} \mathrm{H}_{17} \mathrm{~F}_{5} \mathrm{O}_{3} \mathrm{Na}[\mathrm{M}+\mathrm{Na}]^{+}: 399.0990$; found: 399.0988 .<smiles>O=c1c(OCCC/C=C/CC(F)(F)F)c(-c2ccccc2)oc2ccccc12</smiles>

2z: (E)-3-((7,7,8,8,8-pentafluorooct-4-en-1-yl)oxy)-2-phenyl-4H-chromen-4-one. Prepared according to the general procedure. Reaction was run using $\mathbf{1 z}(96.0 \mathrm{mg}, 0.3 \mathrm{mmol}),\left[\mathrm{CuCF}_{2} \mathrm{CF}_{3}\right](1.0$ $\mathrm{mL}, 0.90 \mathrm{mmol}$ in DMF). The product was purified by flash column chromatography on silica gel (hexane/Et $2 \mathrm{O})$ and obtained a yellow oil $(0.25 \mathrm{mmol}, 110.4 \mathrm{mg}, 84 \%, E / Z=95: 5$, allylic : vinylic $=$ 
$23: 1), \mathrm{R}_{\mathrm{f}}=0.6$ (hexane : $\left.\mathrm{Et}_{2} \mathrm{O}=3: 1\right) .{ }^{1} \mathbf{H} \mathbf{N M R}\left(400 \mathrm{MHz}, \mathrm{CDCl}_{3}\right): \delta 8.23(\mathrm{~d}, J=8.0 \mathrm{~Hz}, 2 \mathrm{H}), 8.08-$ $8.03(\mathrm{~m}, 2 \mathrm{H}), 7.62(\mathrm{t}, J=8.5 \mathrm{~Hz}, 1 \mathrm{H}), 7.52-7.47(\mathrm{~m}, 3 \mathrm{H}), 7.38-7.33(\mathrm{~m}, 1 \mathrm{H}), 5.67-5.59(\mathrm{~m}, 1 \mathrm{H}), 5.29-$ $5.22(\mathrm{~m}, 1 \mathrm{H}), 4.03(\mathrm{t}, J=6.4 \mathrm{~Hz}, 2 \mathrm{H}), 2.67(\mathrm{td}, J=17.6 \mathrm{~Hz}, J=6.8 \mathrm{~Hz}, 2 \mathrm{H}), 2.13$ (q, $J=7.1 \mathrm{~Hz}, 2 \mathrm{H})$, 1.77 (m, 2H) ppm. ${ }^{13} \mathbf{C}$ NMR (101 MHz, $\left.\mathrm{CDCl}_{3}\right): \delta 175.1,156.0,155.3,140.5,137.8,133.4,131.0,130.7$, $128.7,128.4,125.7,124.7,124.2,119.2\left(\mathrm{qt}, J_{C-F}=286.8 \mathrm{~Hz}, J_{C-F}=36.3 \mathrm{~Hz}\right), 118.0,117.1\left(\mathrm{t}, J_{C-F}=4.3\right.$ $\mathrm{Hz}), 114.8\left(\mathrm{tq}, J_{C-F}=252.7 \mathrm{~Hz}, J_{C-F}=37.1 \mathrm{~Hz}\right), 71.8,34.5\left(\mathrm{t}, J_{C-F}=22.4 \mathrm{~Hz}\right), 29.3,28.9$ ppm. ${ }^{19} \mathbf{F}$ NMR $\left(470 \mathrm{MHz}, \mathrm{CDCl}_{3}\right): E$ isomer: $\delta-85.79(\mathrm{~s}, 3 \mathrm{~F}),-118.21(\mathrm{t}, J=17.6 \mathrm{~Hz}, 2 \mathrm{~F}) ; Z$ isomer: $\delta-85.87(\mathrm{~s}, 3 \mathrm{~F}),-$ 117.89 (t, $J=17.9 \mathrm{~Hz}, 2 \mathrm{~F}$ ). HRMS m/z (ESI): calcd. for $\mathrm{C}_{23} \mathrm{H}_{20} \mathrm{~F}_{5} \mathrm{O}_{3}[\mathrm{M}+\mathrm{H}]^{+}: 439.1327$; found: 439.1323 .

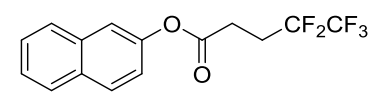

2aa: naphthalen-2-yl 4,4,5,5,5-pentafluoropentanoate. Prepared according to the general procedure. Reaction was run using $1 \mathrm{aa}(59.4 \mathrm{mg}, 0.3 \mathrm{mmol}),\left[\mathrm{CuCF}_{2} \mathrm{CF}_{3}\right](1.0 \mathrm{~mL}, 0.90 \mathrm{mmol}$ in DMF). The product was purified by flash column chromatography on silica gel (hexane/Et $\left.\mathrm{t}_{2} \mathrm{O}\right)$ and obtained a colorless oil (0.06 mmol, $20.0 \mathrm{mg}, 21 \%), \mathrm{R}_{\mathrm{f}}=0.7$ (hexane : $\left.\mathrm{Et}_{2} \mathrm{O}=5: 1\right) .{ }^{1} \mathbf{H} \mathbf{N M R}\left(100 \mathrm{MHz}, \mathrm{CDCl}_{3}\right): \delta 7.89-7.81$ $(\mathrm{m}, 3 \mathrm{H}), 7.58(\mathrm{~d}, J=2.0 \mathrm{~Hz}, 1 \mathrm{H}), 7.54-7.46(\mathrm{~m}, 2 \mathrm{H}), 7.23(\mathrm{dd}, J=8.8 \mathrm{~Hz}, J=2.0 \mathrm{~Hz}, 1 \mathrm{H}), 2.96(\mathrm{t}, J=$ $7.8 \mathrm{~Hz}, 2 \mathrm{H}), 2.65-2.52(\mathrm{~m}, 2 \mathrm{H}) \mathrm{ppm} .{ }^{13} \mathbf{C}$ NMR $\left(126 \mathrm{MHz}, \mathrm{CDCl}_{3}\right): \delta 170.0,148.1,133.8,132.7,129.7$, $127.9,127.8,126.9,126.1,120.8,119.1\left(\mathrm{qt}, J_{C-F}=285.6 \mathrm{~Hz}, J_{C-F}=35.9 \mathrm{~Hz}\right), 118.6,115.3\left(\mathrm{tq}, J_{C-F}=\right.$ $\left.252.8 \mathrm{~Hz}, J_{C-F}=38.2 \mathrm{~Hz}\right), 26.4\left(\mathrm{t}, J_{C-F}=22.1 \mathrm{~Hz}\right), 26.1\left(\mathrm{t}, J_{C-F}=3.8 \mathrm{~Hz}\right) \mathrm{ppm} .{ }^{19} \mathbf{F} \mathbf{N M R}(470 \mathrm{MHz}$, $\left.\mathrm{CDCl}_{3}\right): \delta-86.39(\mathrm{~s}, 3 \mathrm{~F}),-119.57(\mathrm{t}, J=18.1 \mathrm{~Hz}, 2 \mathrm{~F})$. HRMS m/z (ESI): calcd. for $\mathrm{C}_{15} \mathrm{H}_{11} \mathrm{~F}_{5} \mathrm{O}_{2} \mathrm{Na}$ $[\mathrm{M}+\mathrm{Na}]^{+}: 341.0571$; found: 341.0570 .

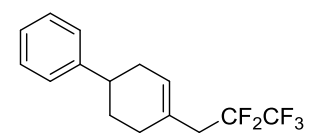

2ab: (4-(2,2,3,3,3-pentafluoropropylidene)cyclohexyl)benzene. Prepared according to the general procedure. Reaction was run using $1 \mathbf{a b}(51.6 \mathrm{mg}, 0.3 \mathrm{mmol}),\left[\mathrm{CuCF}_{2} \mathrm{CF}_{3}\right](1.0 \mathrm{~mL}, 0.90 \mathrm{mmol}$ in DMF). The product was purified by flash column chromatography on silica gel (hexane) and obtained a colorless oil ( $0.03 \mathrm{mmol}, 8.7 \mathrm{mg}, 10 \%), \mathrm{R}_{\mathrm{f}}=0.5$ (hexane). ${ }^{1} \mathbf{H} \mathbf{N M R}\left(500 \mathrm{MHz}, \mathrm{CDCl}_{3}\right): \delta 7.31$ (t, $J=7.5 \mathrm{~Hz}$, $2 \mathrm{H}), 7.25-7.21(\mathrm{~m}, 3 \mathrm{H}), 5.77(\mathrm{~s}, 1 \mathrm{H}), 2.81-2.68(\mathrm{~m}, 3 \mathrm{H}), 2.38-2.27(\mathrm{~m}, 2 \mathrm{H}), 2.23-2.15(\mathrm{~m}, 2 \mathrm{H}), 1.98(\mathrm{dd}$, $J=12.0 \mathrm{~Hz}, J=2.0 \mathrm{~Hz}, 1 \mathrm{H}), 1.79(\mathrm{qd}, J=12.0 \mathrm{~Hz}, J=5.5 \mathrm{~Hz}, 1 \mathrm{H}) \mathrm{ppm} .{ }^{13} \mathbf{C} \mathbf{N M R}\left(126 \mathrm{MHz}, \mathrm{CDCl}_{3}\right)$ : $\delta$ 146.7, 129.7, 128.6, 127.0, 126.3, $119.3\left(\mathrm{qt}, J_{C-F}=286.4 \mathrm{~Hz}, J_{C-F}=36.2 \mathrm{~Hz}\right), 115.4\left(\mathrm{tq}, J_{C-F}=252.5\right.$ $\mathrm{Hz}, J_{C-F}=36.9 \mathrm{~Hz}$ ), 39.5, $38.9\left(\mathrm{t}, J_{C-F}=21.7 \mathrm{~Hz}\right.$ ), 33.8, 30.0, $30.0 \mathrm{ppm}$. (one carbon missing due to overlap) ${ }^{19} \mathbf{F}$ NMR (470 MHz, $\mathrm{CDCl}_{3}$ ): $\delta-86.05$ (s, 3F), -116.98 (m, 2F). HRMS m/z (APCI): calcd. for $\mathrm{C}_{15} \mathrm{H}_{16} \mathrm{~F}_{5}[\mathrm{M}+\mathrm{H}]^{+}: 291.1167$; found: 291.1167 .

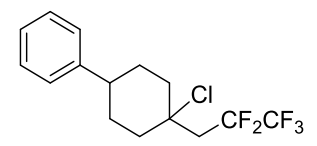

2ab": (4-chloro-4-(2,2,3,3,3-pentafluoropropyl)cyclohexyl)benzene. Prepared according to the general procedure. Reaction was run using $1 \mathbf{a b}(51.6 \mathrm{mg}, 0.3 \mathrm{mmol}),\left[\mathrm{CuCF}_{2} \mathrm{CF}_{3}\right](1.0 \mathrm{~mL}, 0.90 \mathrm{mmol}$ in DMF). The product was purified by flash column chromatography on silica gel (hexane) and obtained a white solid, melting point: $71.3-71.8^{\circ} \mathrm{C}(0.25 \mathrm{mmol}, 82.2 \mathrm{mg}, 84 \%), \mathrm{R}_{\mathrm{f}}=0.4$ (hexane). ${ }^{1} \mathrm{H}$ NMR (400 
MHz, $\left.\mathrm{CDCl}_{3}\right): \delta 7.30(\mathrm{~d}, J=7.4 \mathrm{~Hz}, 2 \mathrm{H}), 7.24(\mathrm{~d}, J=7.2 \mathrm{~Hz}, 2 \mathrm{H}), 7.20(\mathrm{t}, J=7.4 \mathrm{~Hz}, 1 \mathrm{H}), 2.65(\mathrm{t}, J=$ $19.6 \mathrm{~Hz}, 2 \mathrm{H}), 2.50(\mathrm{tt}, J=12.0 \mathrm{~Hz}, J=3.6 \mathrm{~Hz}, 1 \mathrm{H}), 2.22(\mathrm{~d}, J=12.4 \mathrm{~Hz}, 2 \mathrm{H}), 2.07$ (q, $J=12.4 \mathrm{~Hz}, 2 \mathrm{H})$, $1.93(\mathrm{t}, J=13.2 \mathrm{~Hz}, 2 \mathrm{H}), 1.81(\mathrm{~d}, J=11.6 \mathrm{~Hz}, 2 \mathrm{H}) \mathrm{ppm} .{ }^{13} \mathbf{C} \mathbf{N M R}\left(126 \mathrm{MHz}, \mathrm{CDCl}_{3}\right): \delta 146.3,128.6$, $127.0,126.5,118.9\left(\mathrm{qt}, J_{C-F}=286.8 \mathrm{~Hz}, J_{C-F}=35.7 \mathrm{~Hz}\right), 115.4$ (tq, $\left.J_{C-F}=257.0 \mathrm{~Hz}, J_{C-F}=37.3 \mathrm{~Hz}\right), 69.2$, $44.6\left(\mathrm{t}, J_{C-F}=19.4 \mathrm{~Hz}\right), 43.2,39.8,29.4 \mathrm{ppm} .{ }^{19} \mathbf{F} \mathbf{N M R}\left(470 \mathrm{MHz}, \mathrm{CDCl}_{3}\right): \delta-87.46(\mathrm{~s}, 3 \mathrm{~F}),-116.10(\mathrm{t}$, $J=19.5 \mathrm{~Hz}, 2 \mathrm{~F}$ ). HRMS m/z (APCI): calcd. for $\mathrm{C}_{15} \mathrm{H}_{16} \mathrm{ClF}_{5}[\mathrm{M}]^{+}: 326.0855$; found: 326.0860 . 
Spectra:

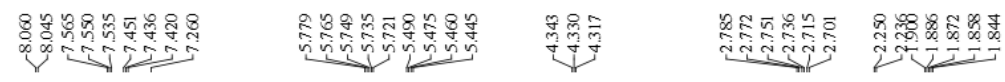

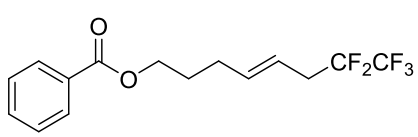

2a ( ${ }^{1} \mathrm{H}$ NMR $\left.\mathrm{CDCl}_{3}, 500 \mathrm{MHz}\right)$
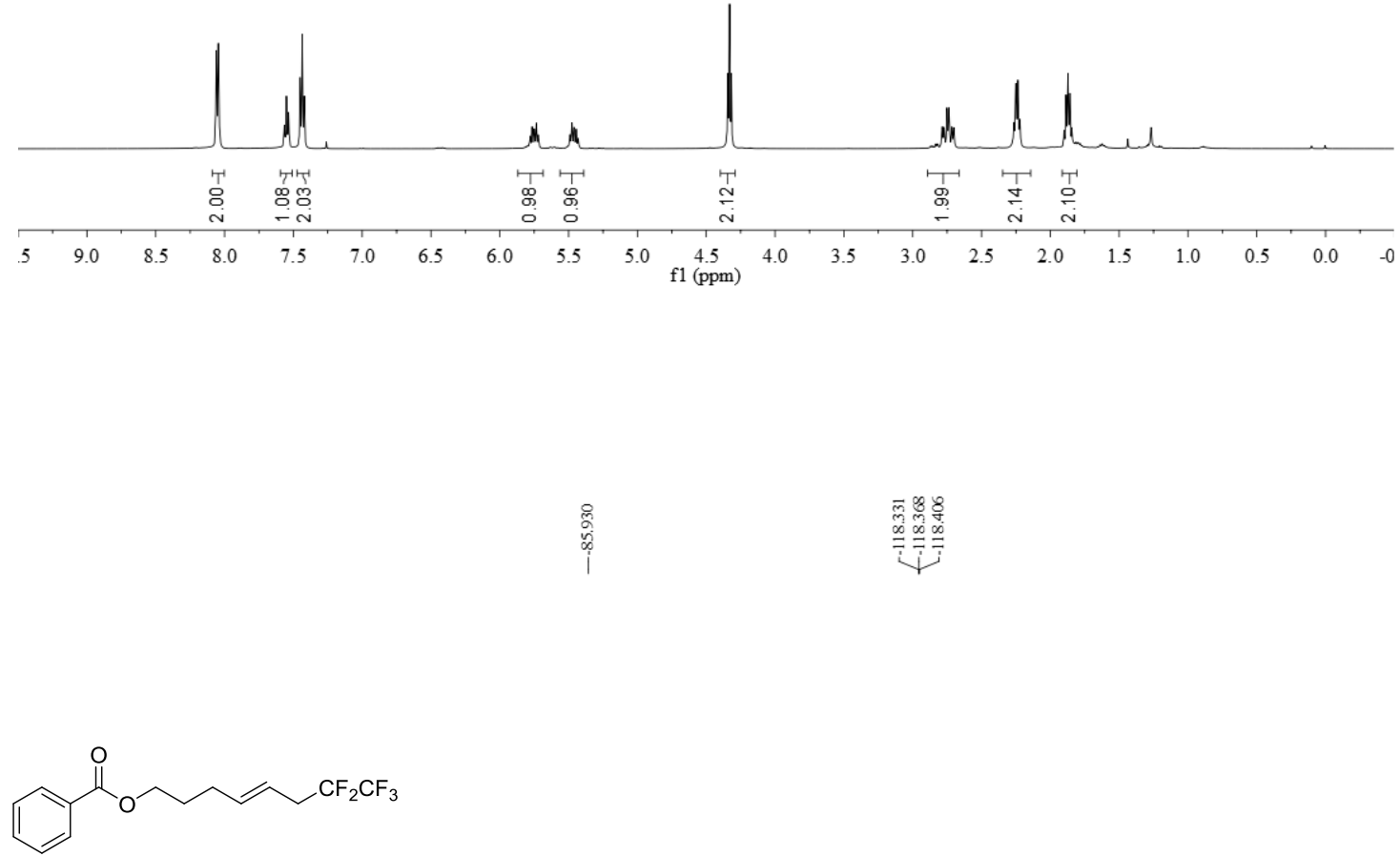

2a $\left({ }^{19} \mathrm{~F}\right.$ NMR, $\left.\mathrm{CDCl}_{3}, 470 \mathrm{MHz}\right)$

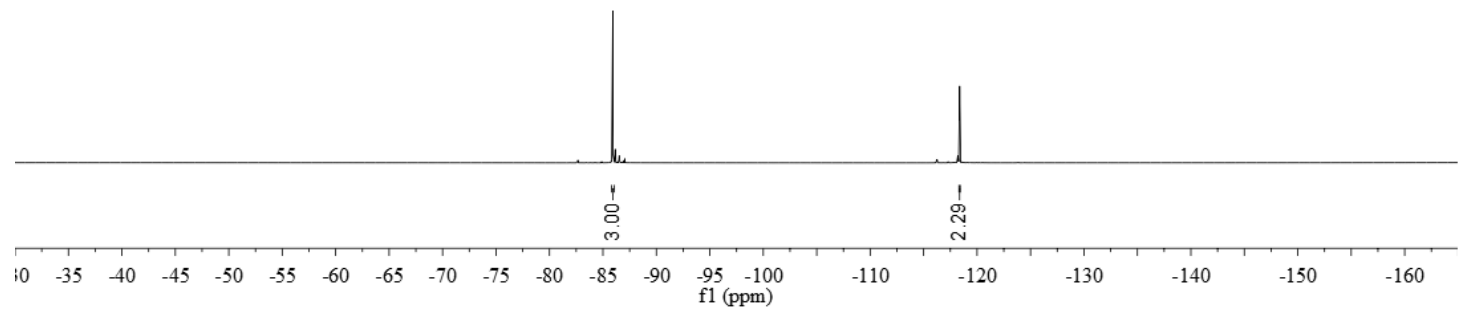




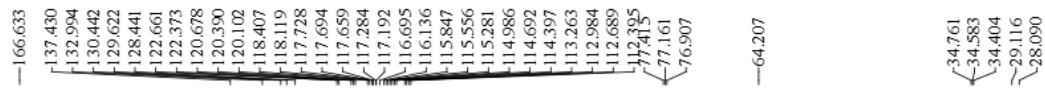
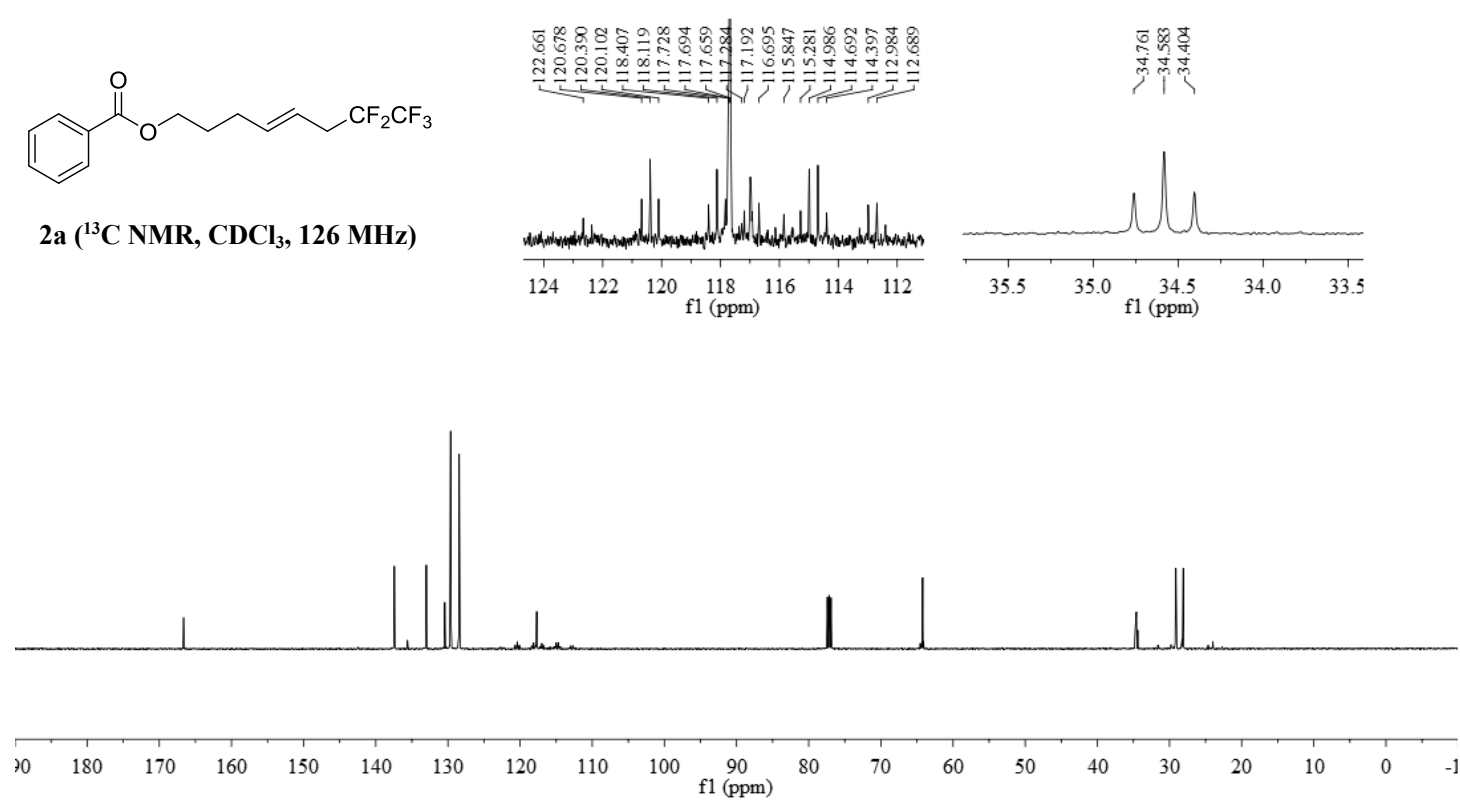

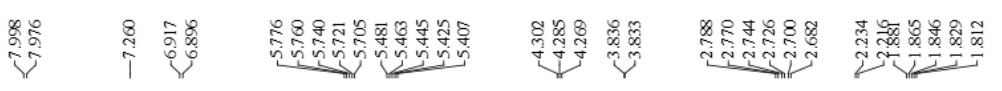
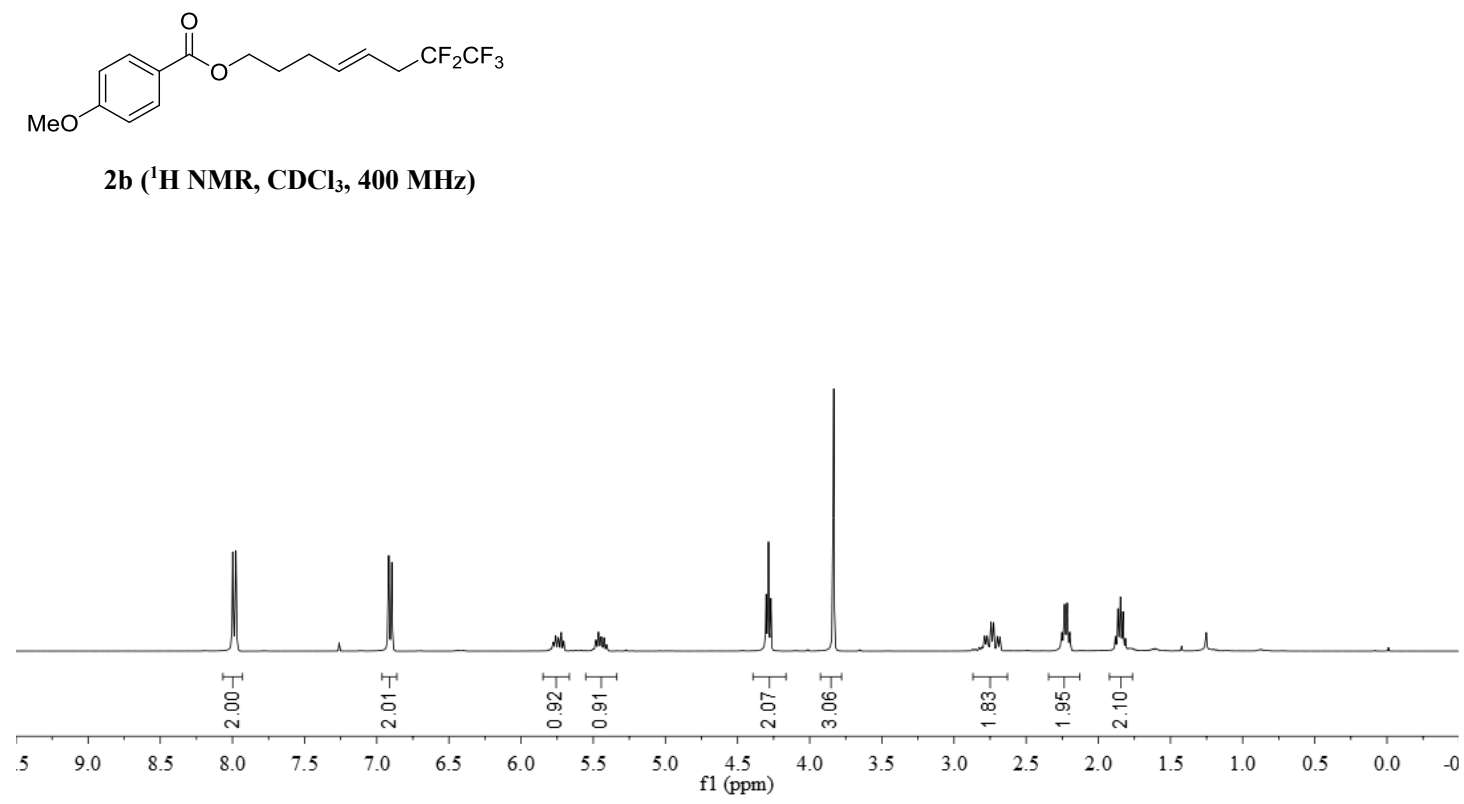

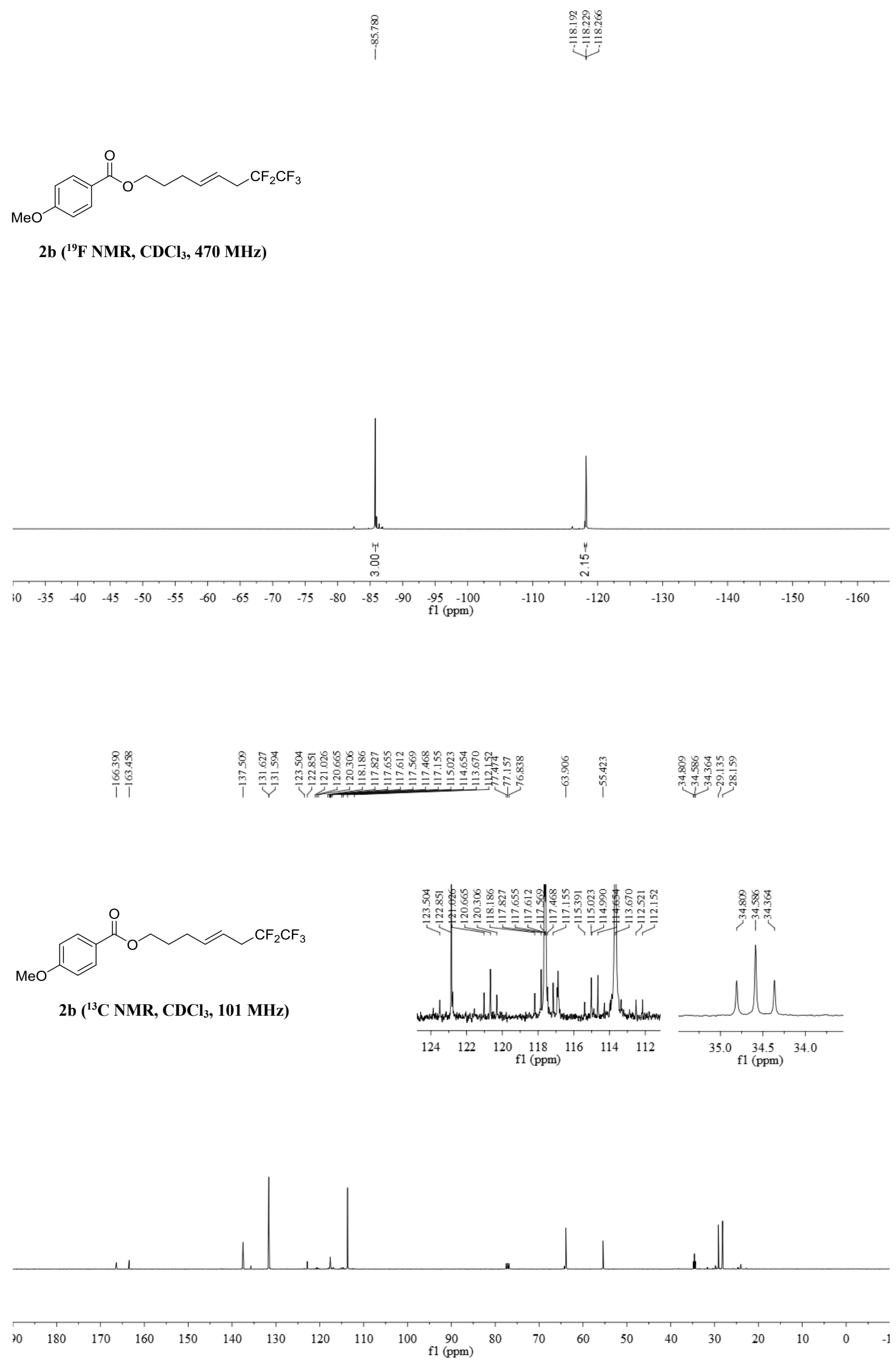


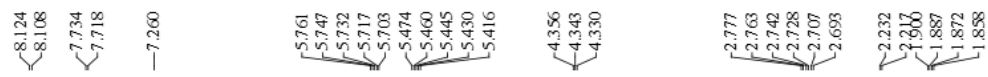
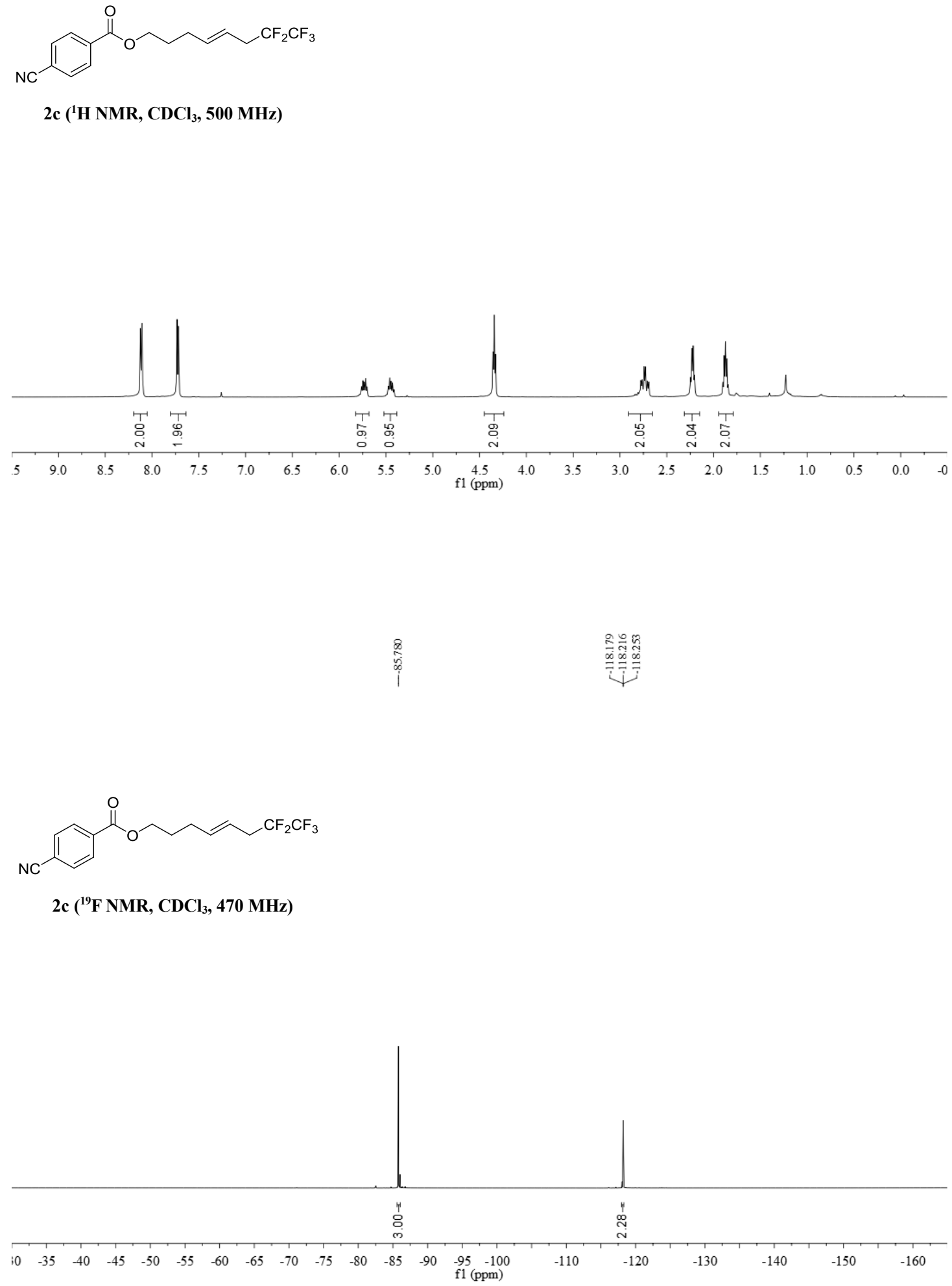

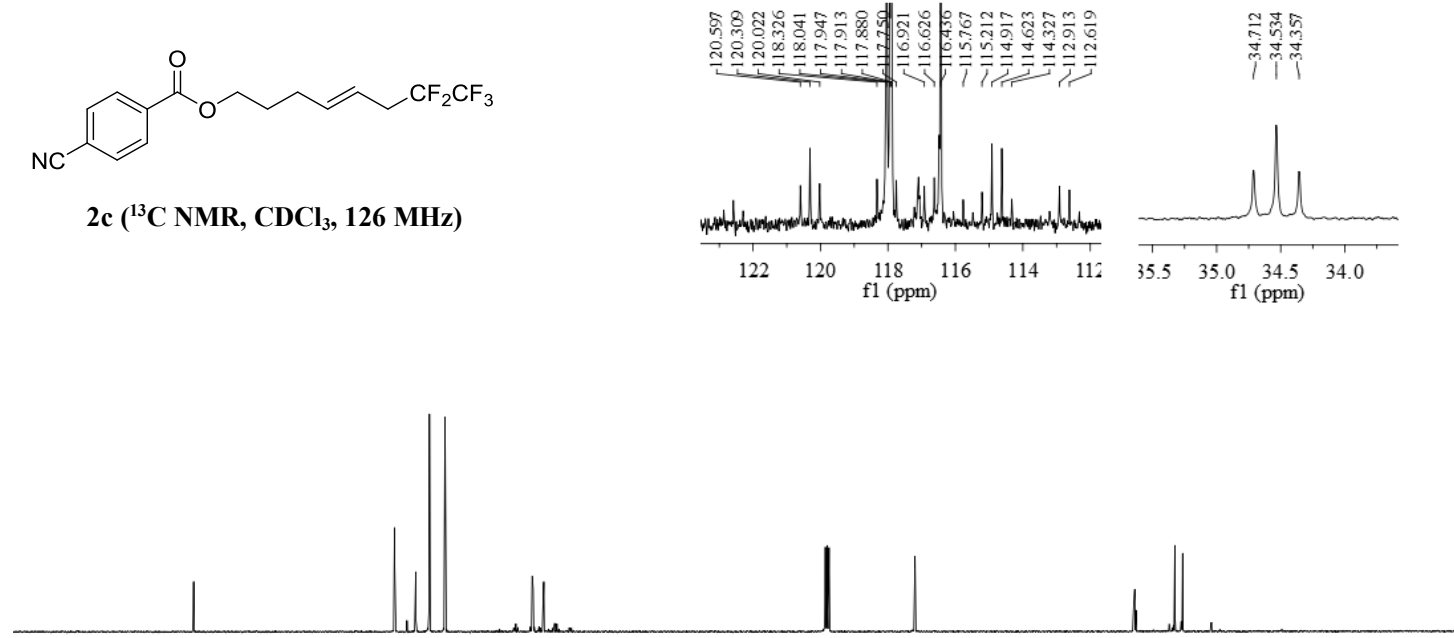

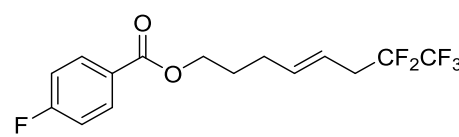

2d ( ${ }^{1} \mathrm{H}$ NMR, $\left.\mathrm{CDCl}_{3}, 500 \mathrm{MHz}\right)$

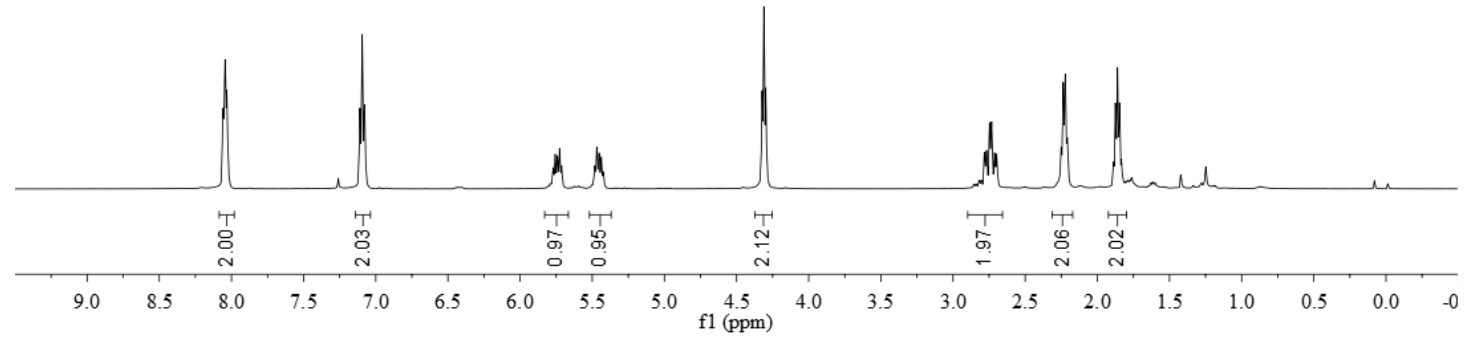




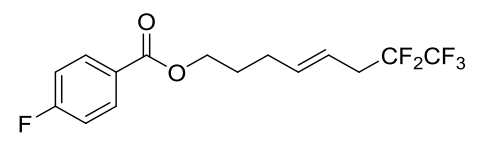

2d ( $\left.{ }^{19} \mathrm{~F} \mathrm{NMR,} \mathrm{CDCl}_{3}, 470 \mathrm{MHz}\right)$
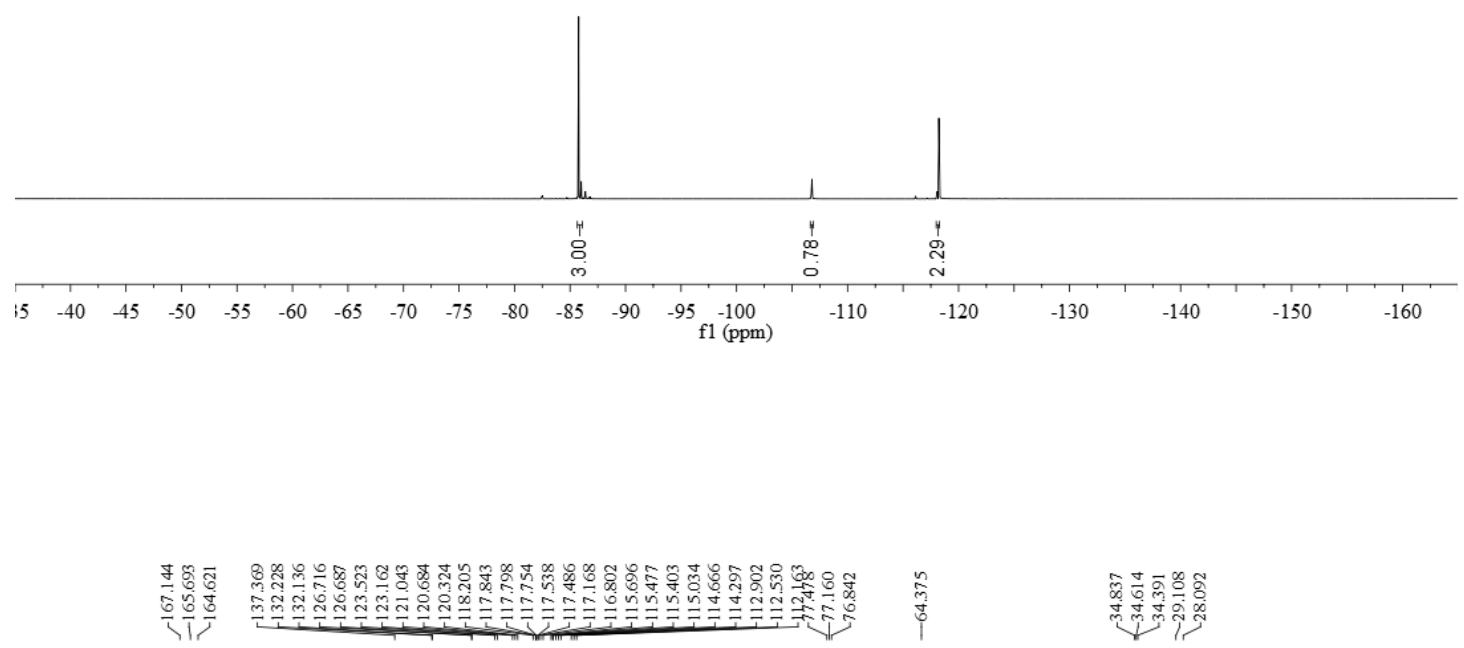

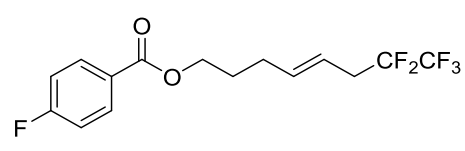

2d $\left({ }^{13} \mathrm{C} \mathrm{NMR,} \mathrm{CDCl}_{3}, 126 \mathrm{MHz}\right)$

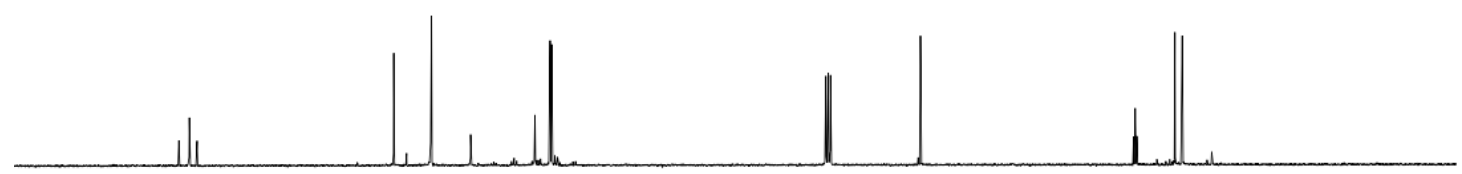

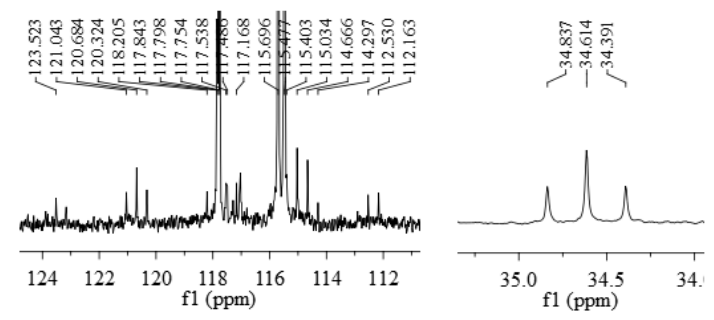

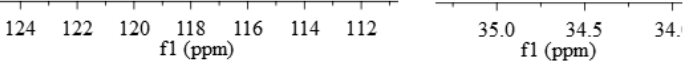

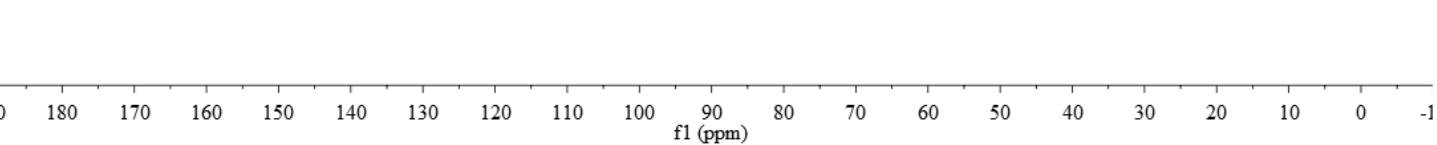




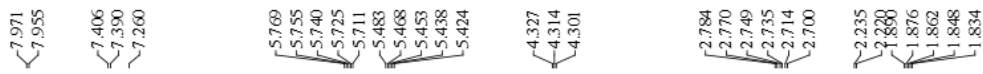
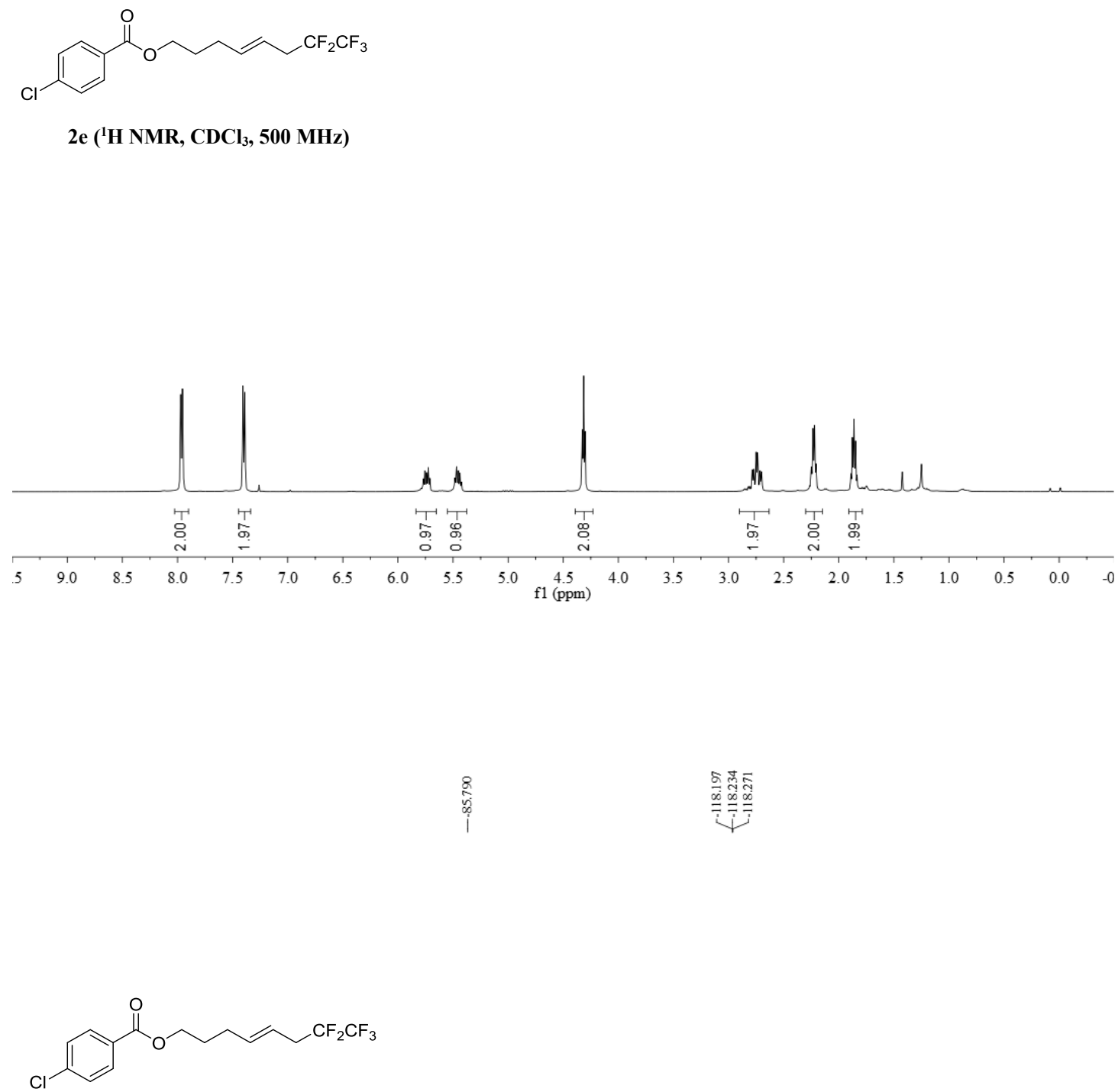

2e ( ${ }^{19} \mathrm{~F}$ NMR, $\left.\mathrm{CDCl}_{3}, 470 \mathrm{MHz}\right)$

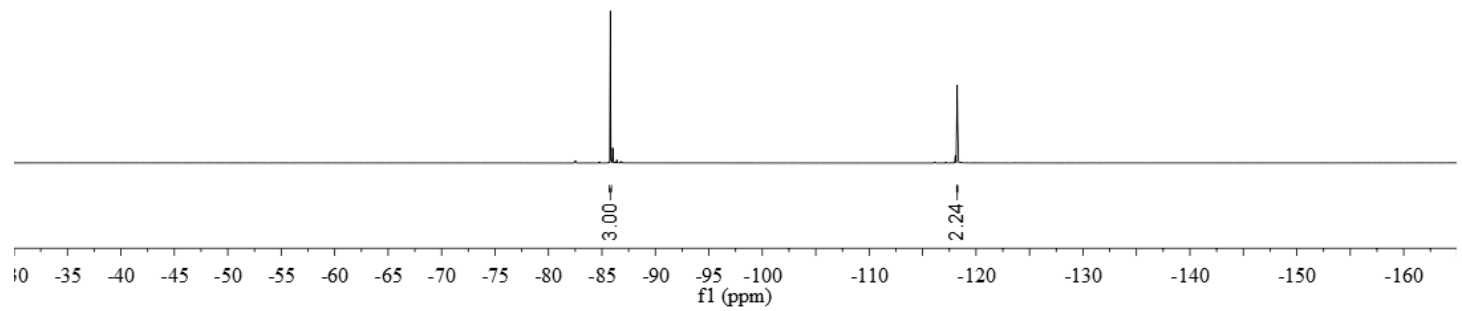




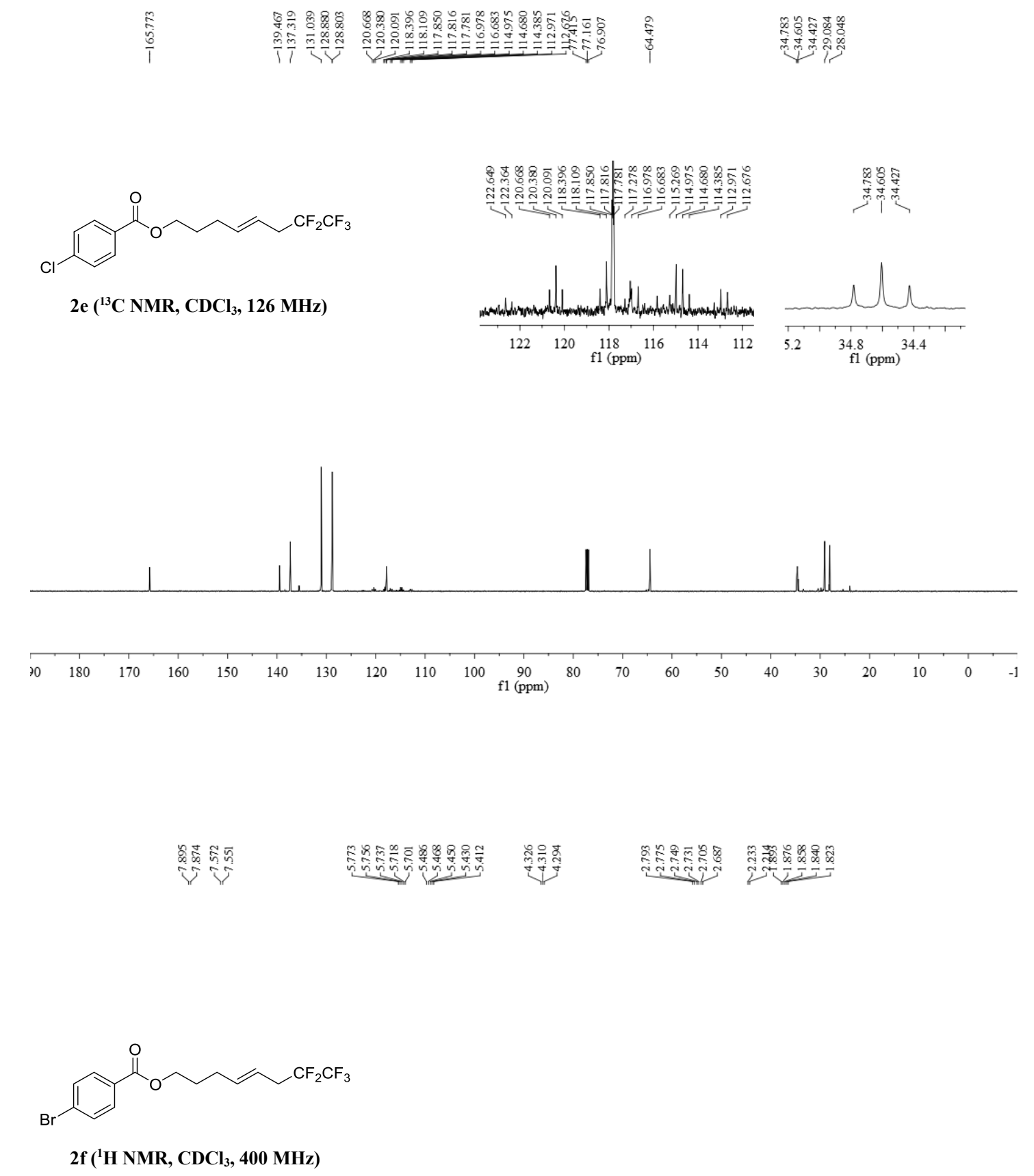

2f ( ${ }^{1} \mathrm{H}$ NMR, $\left.\mathrm{CDCl}_{3}, 400 \mathrm{MHz}\right)$

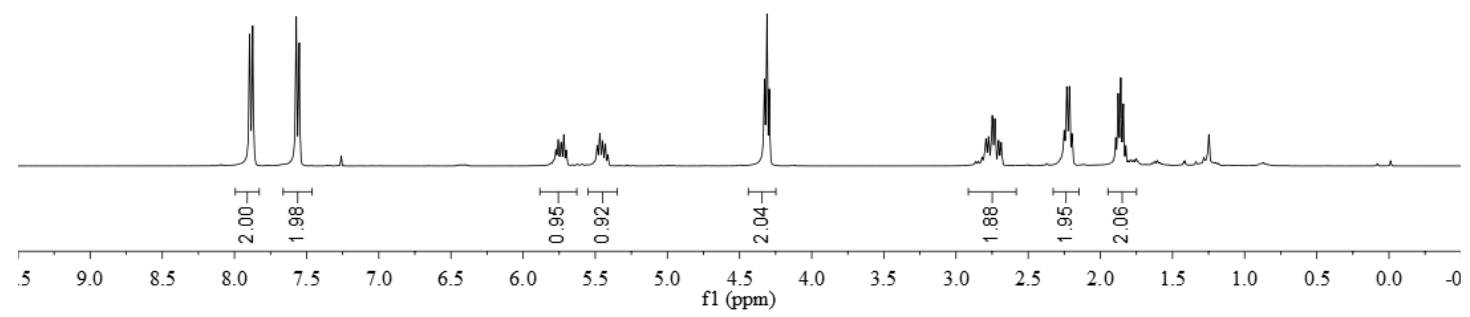



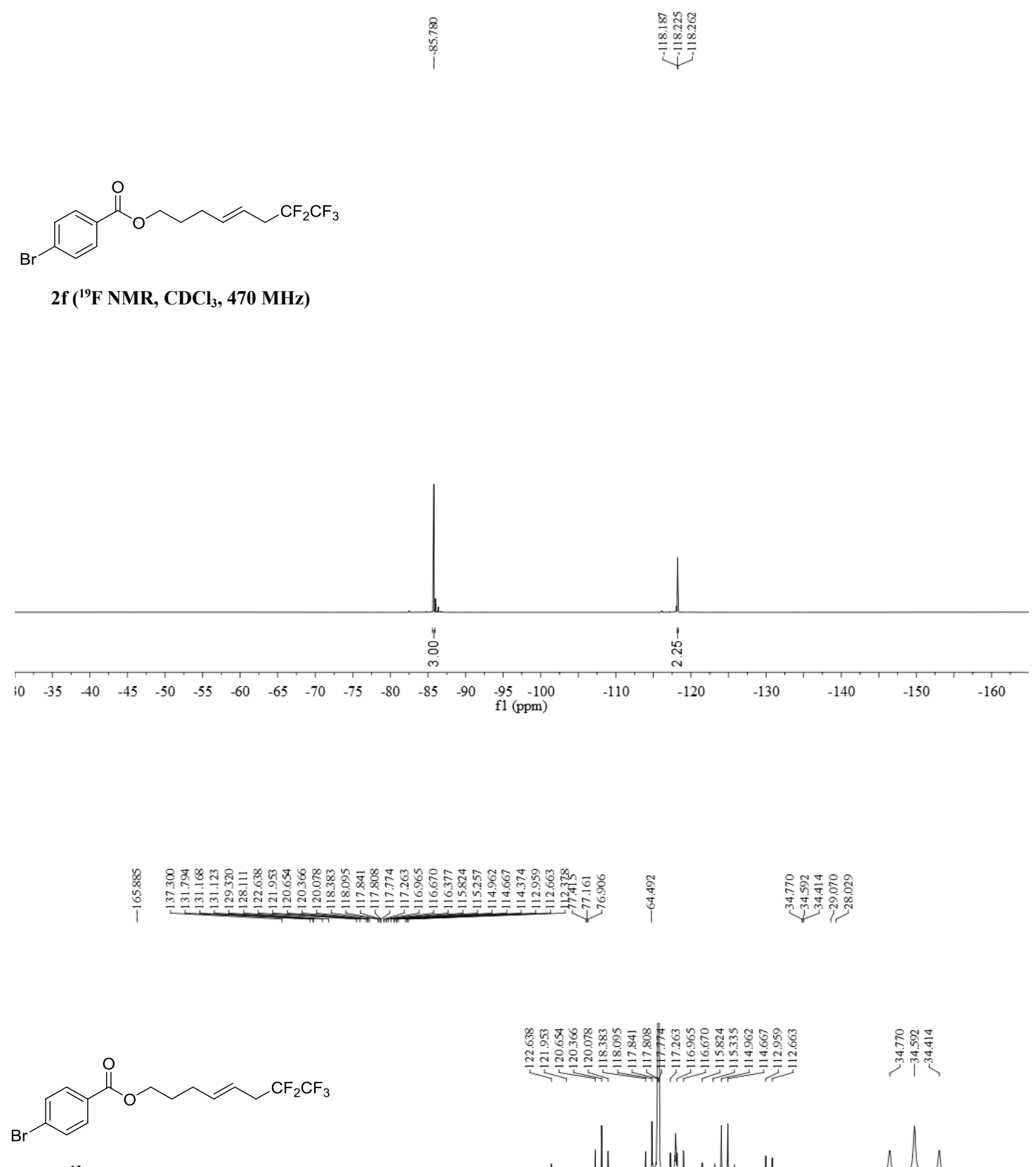

2f $\left({ }^{13} \mathrm{C}\right.$ NMR, $\left.\mathrm{CDCl}_{3}, 126 \mathrm{MHz}\right)$

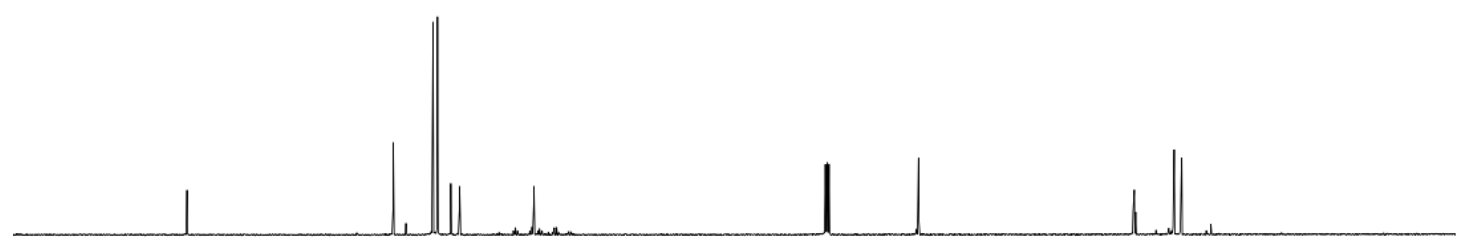

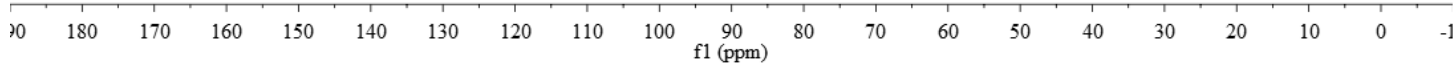



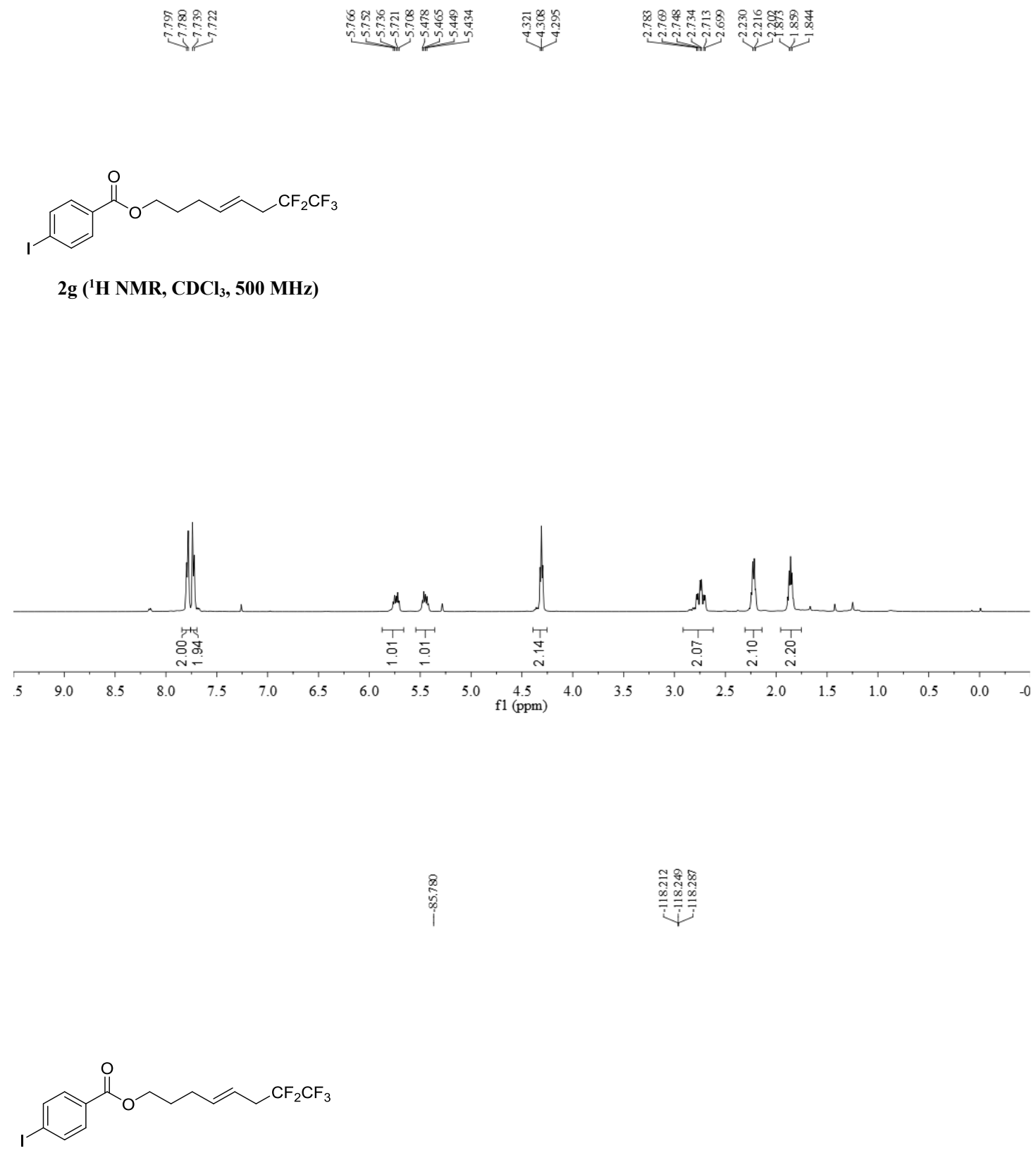

$2 \mathrm{~g}\left({ }^{19} \mathrm{~F} \mathrm{NMR}, \mathrm{CDCl}_{3}, 470 \mathrm{MHz}\right)$

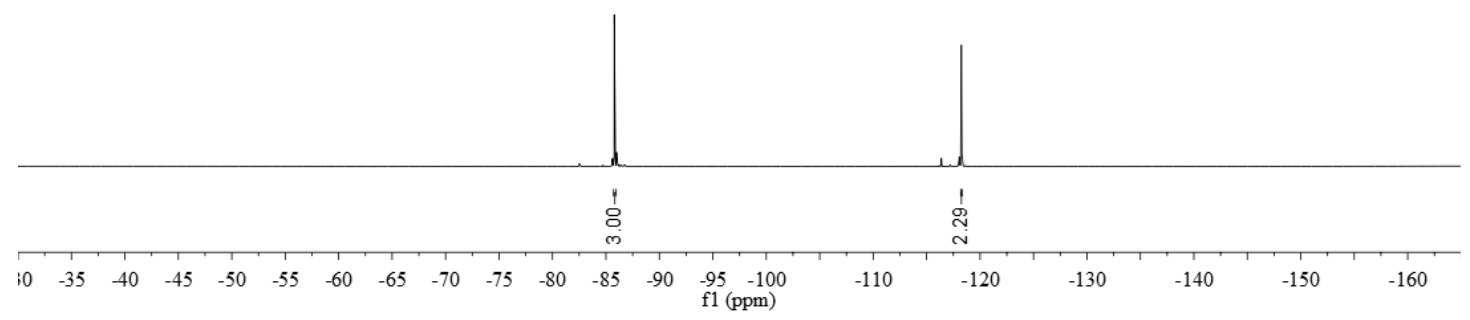




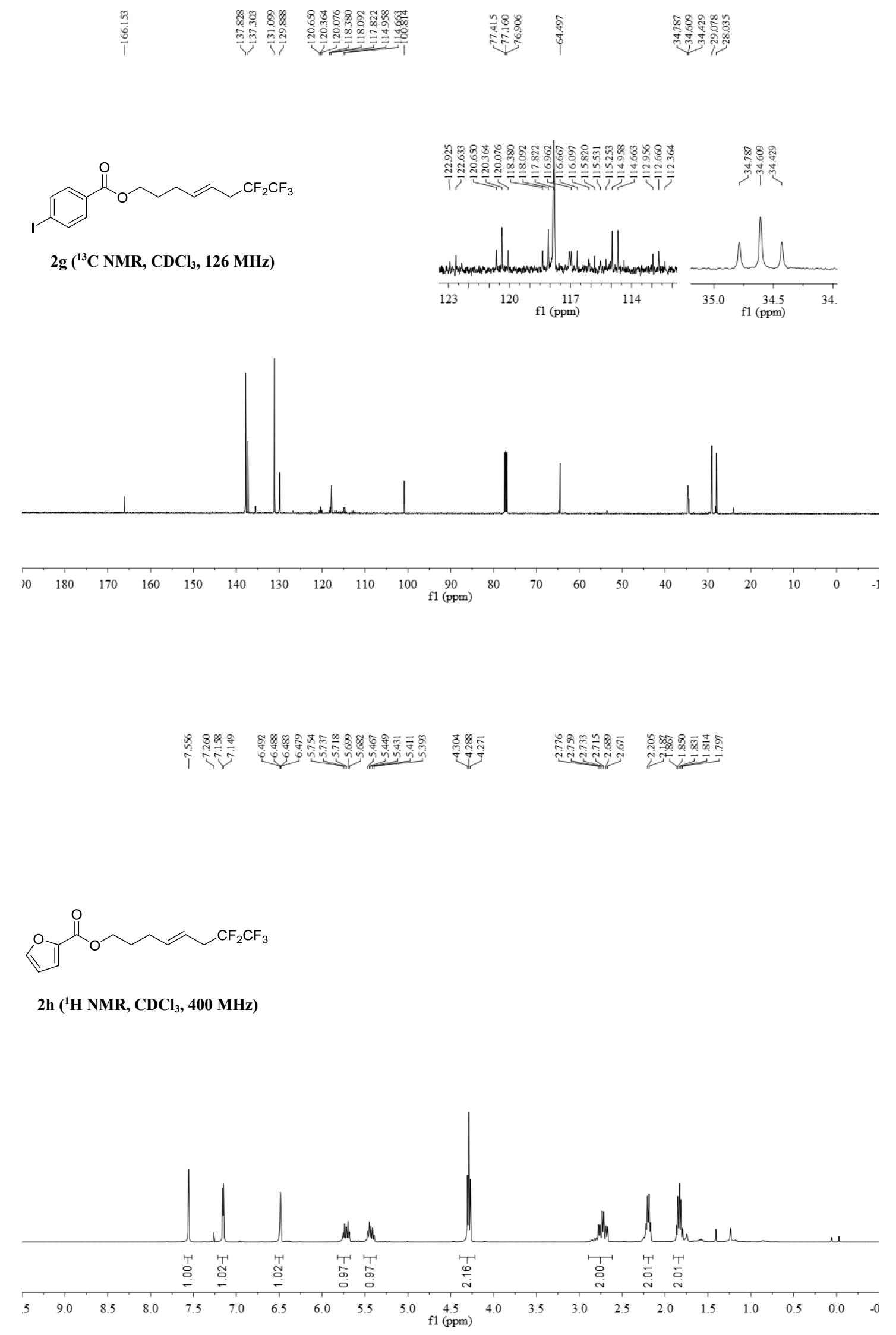


$\underset{\substack{8 \\ 0}}{\substack{0 \\ 1}}$

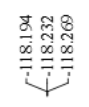

(II

Th $\left({ }^{19} \mathrm{~F}\right.$ NMR, $\left.\mathrm{CDCl}_{3}, 470 \mathrm{MHz}\right)$
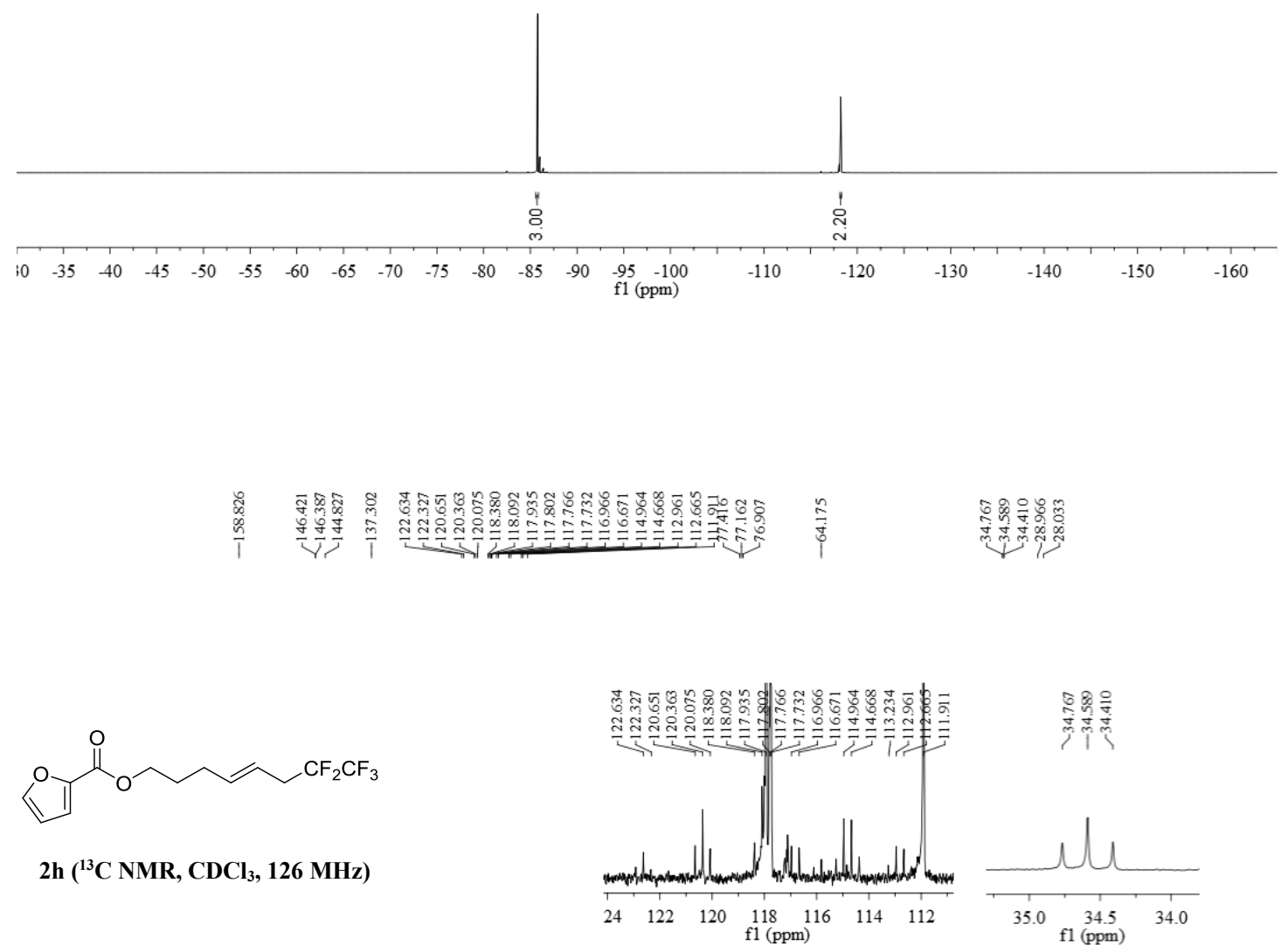

籍最

Th $\left({ }^{13} \mathrm{C}\right.$ NMR, $\left.\mathrm{CDCl}_{3}, 126 \mathrm{MHz}\right)$
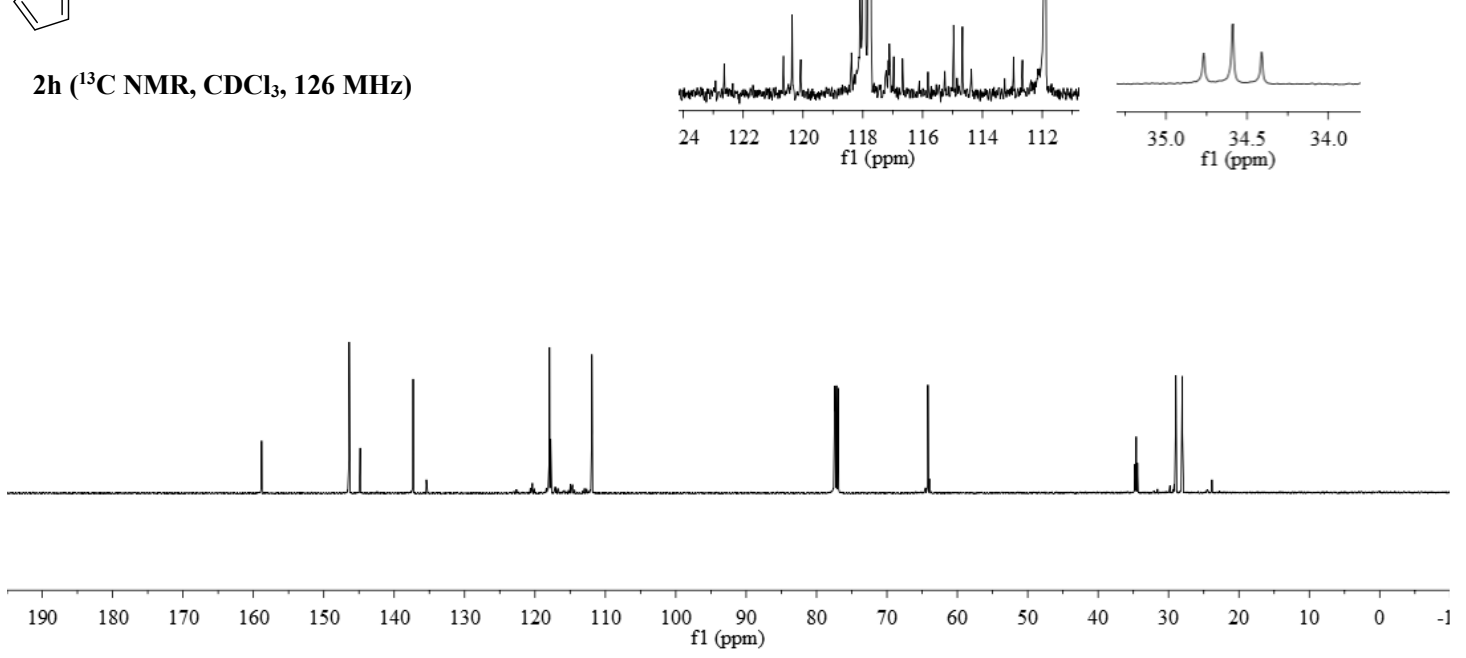

S35 


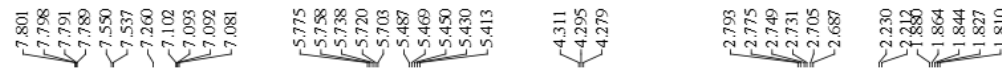

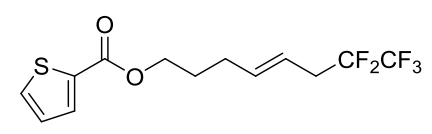

2i ( ${ }^{1} \mathrm{H}$ NMR, $\left.\mathrm{CDCl}_{3}, 400 \mathrm{MHz}\right)$
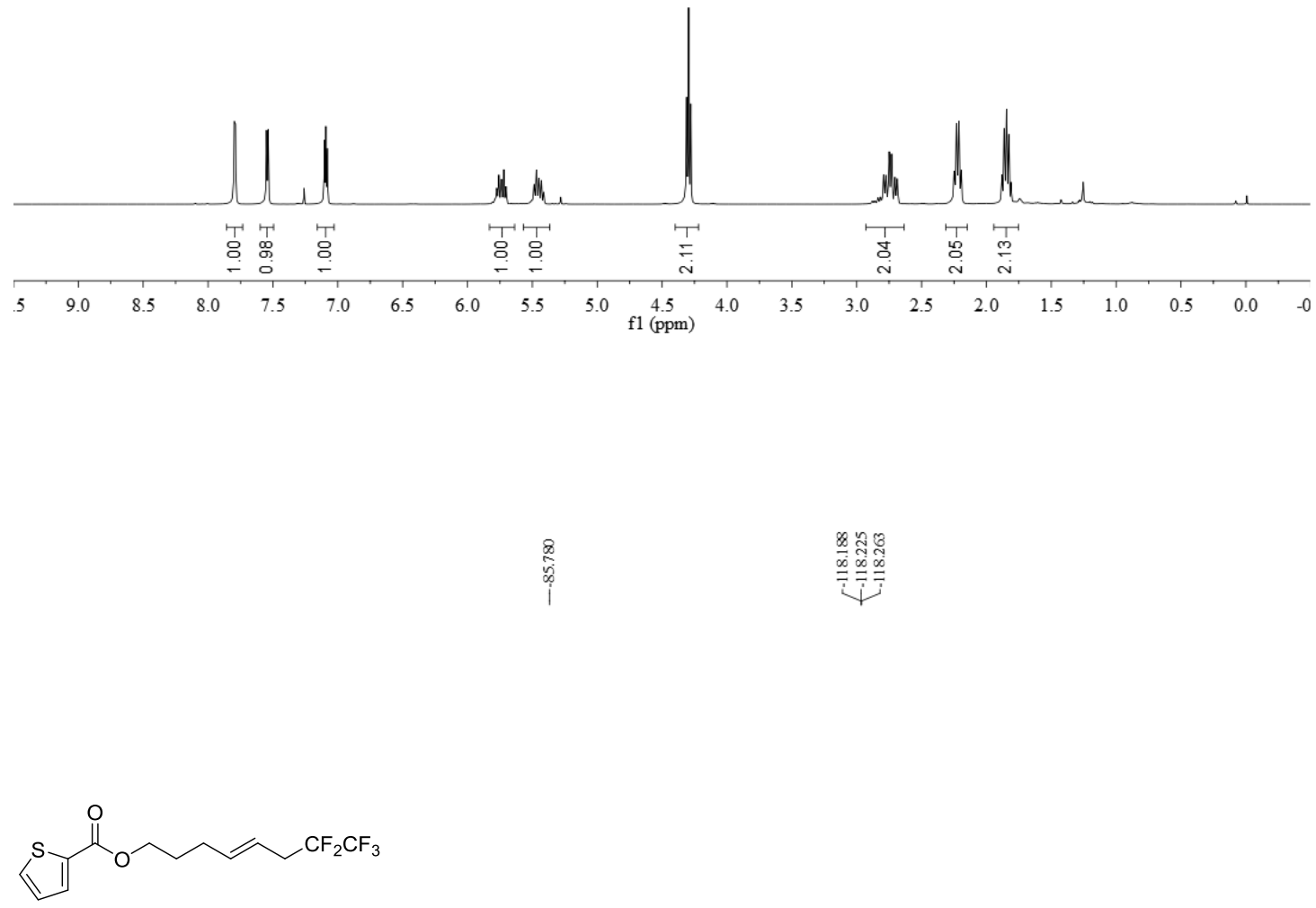

2i $\left({ }^{19} \mathrm{~F}\right.$ NMR, $\left.\mathrm{CDCl}_{3}, 470 \mathrm{MHz}\right)$

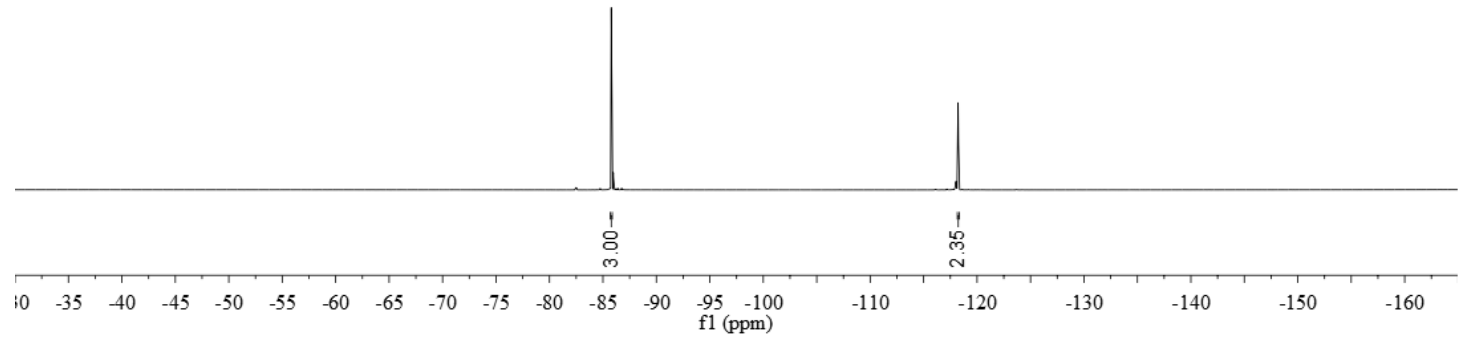




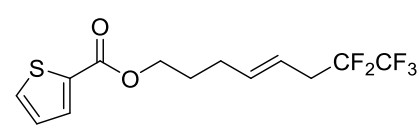

2i $\left({ }^{13} \mathrm{C}\right.$ NMR, $\left.\mathrm{CDCl}_{3}, 101 \mathrm{MHz}\right)$
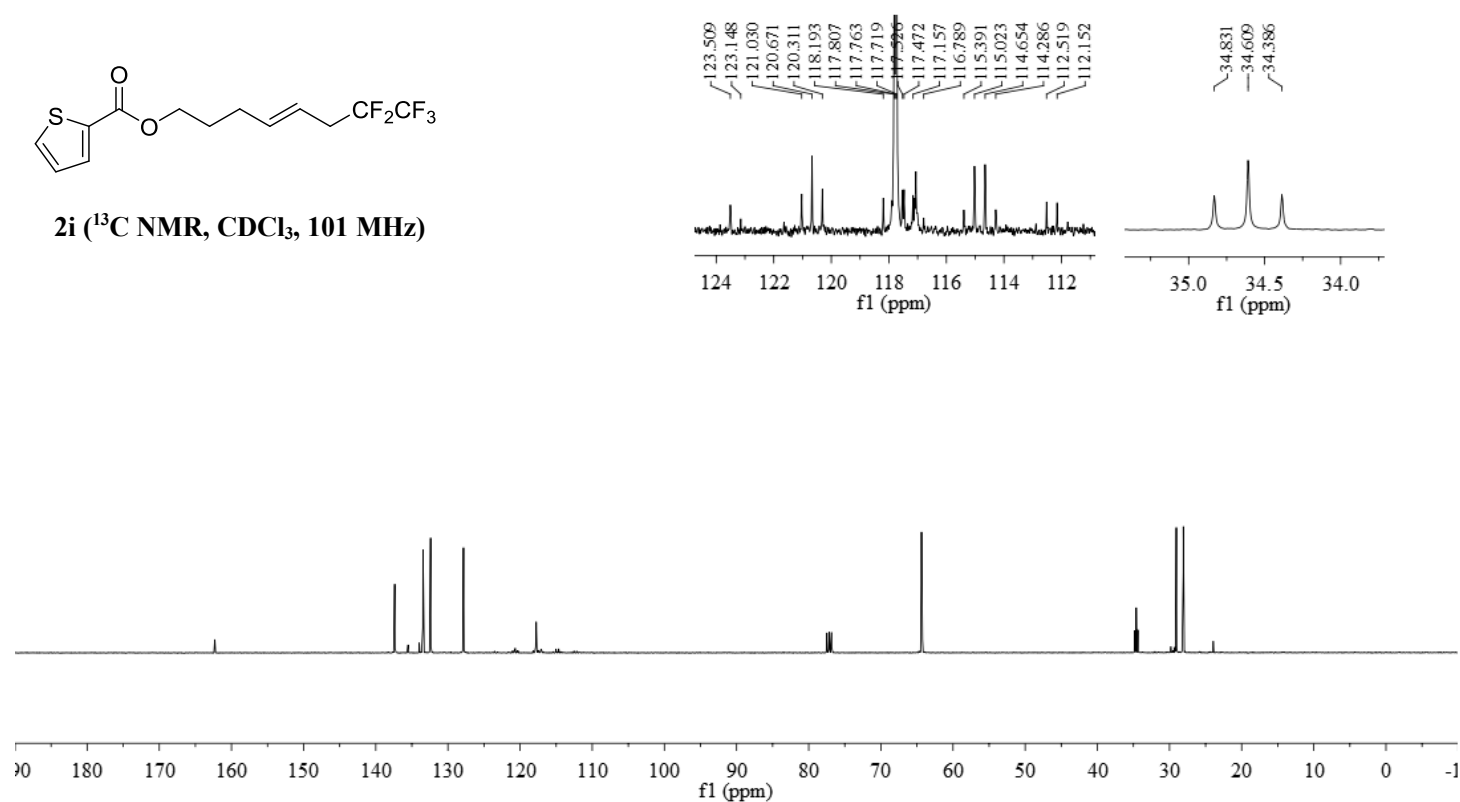

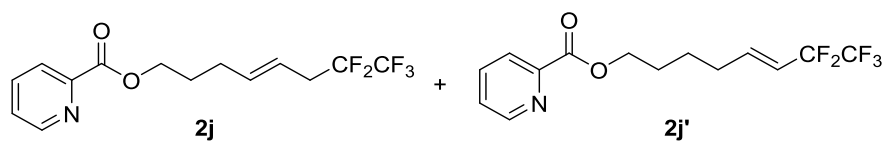

$2 \mathrm{j}: 2 \mathrm{j}^{\prime}=9$ : 1

('H NMR, $\mathrm{CDCl}_{3}, 400 \mathrm{MHz}$ )

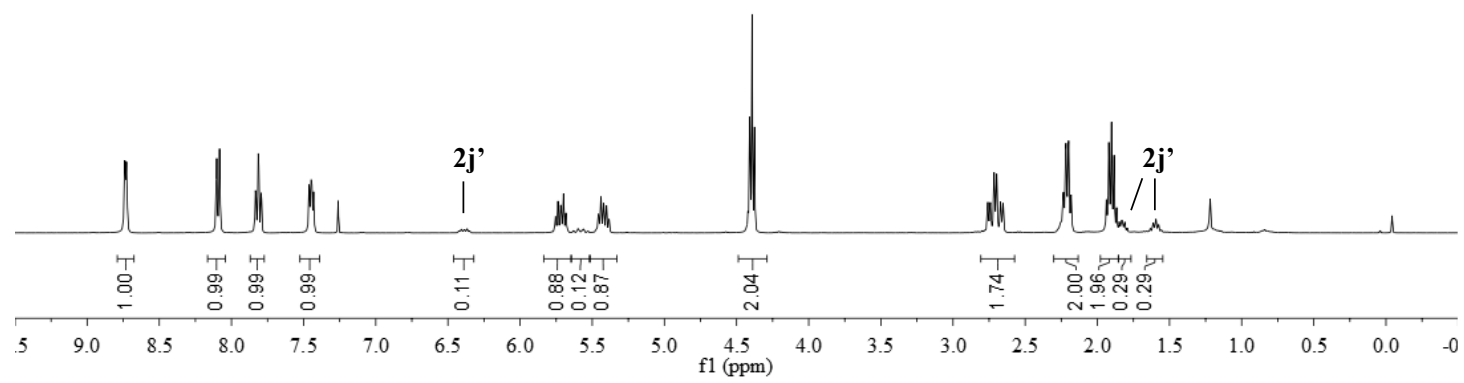




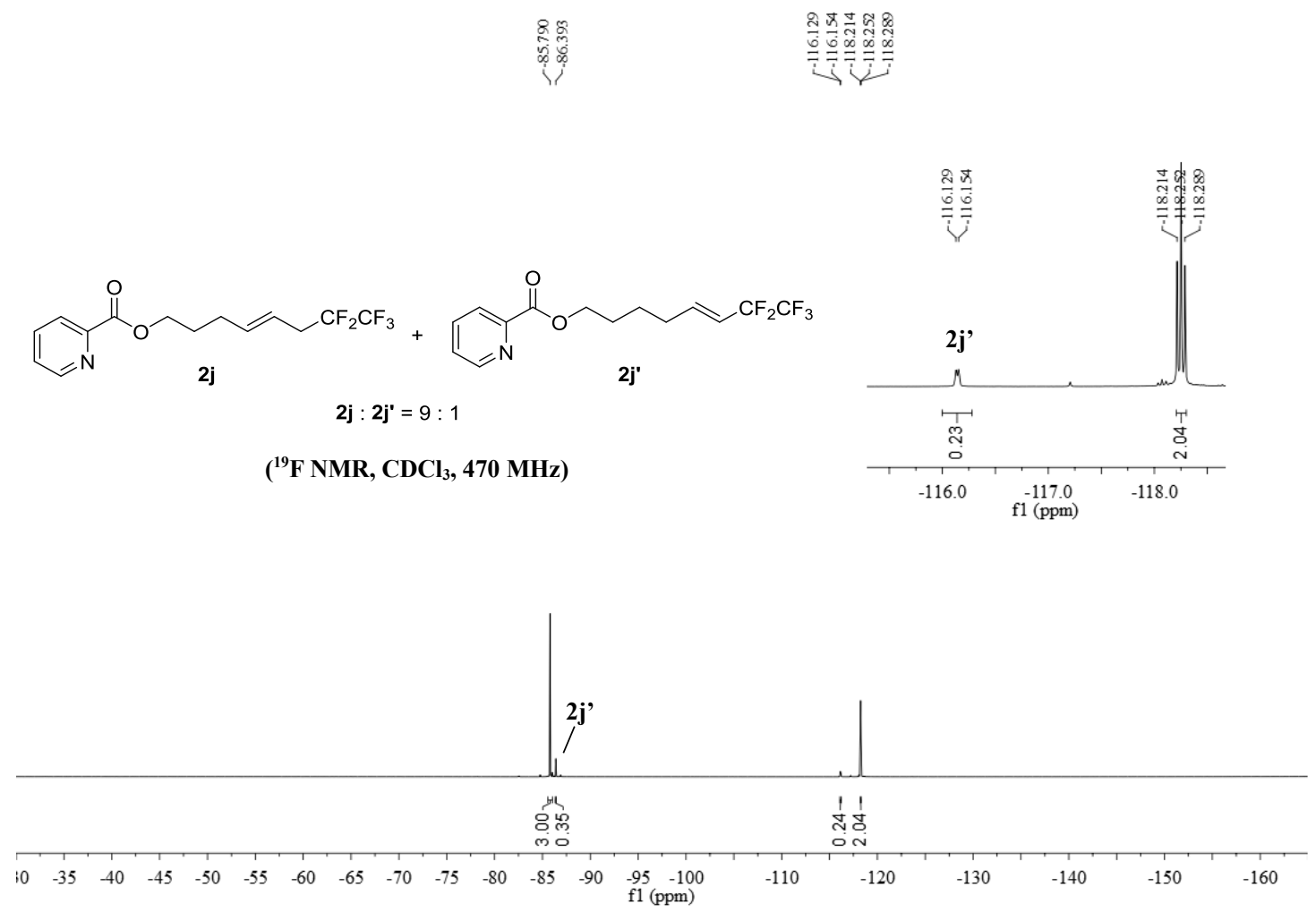

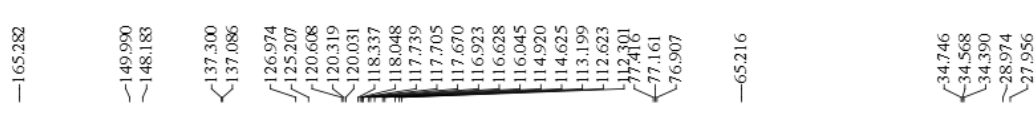

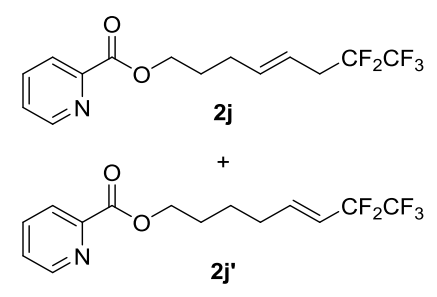

$\mathbf{2 j}: \mathbf{2} \mathbf{j}^{\prime}=9: 1$

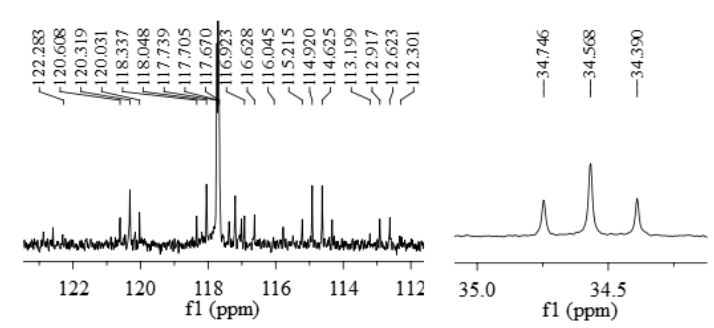

$\left({ }^{13} \mathrm{C} \mathrm{NMR}, \mathrm{CDCl}_{3}, 126 \mathrm{MHz}\right)$

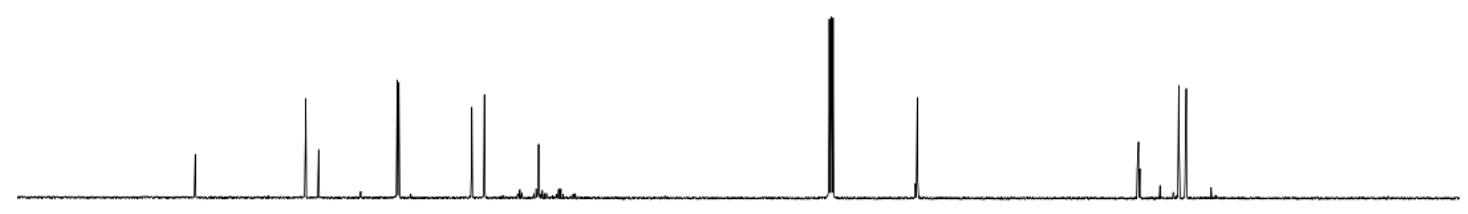

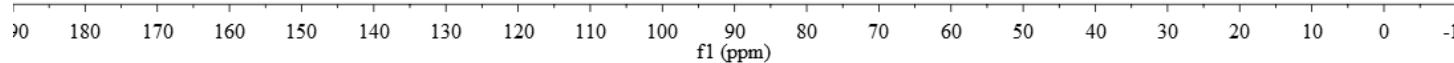




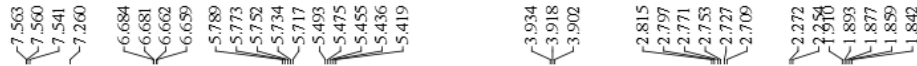

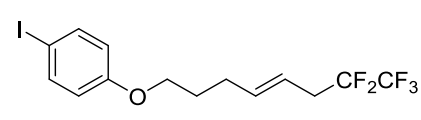

2k ( ${ }^{1} \mathrm{H}$ NMR, $\left.\mathrm{CDCl}_{3}, 400 \mathrm{MHz}\right)$
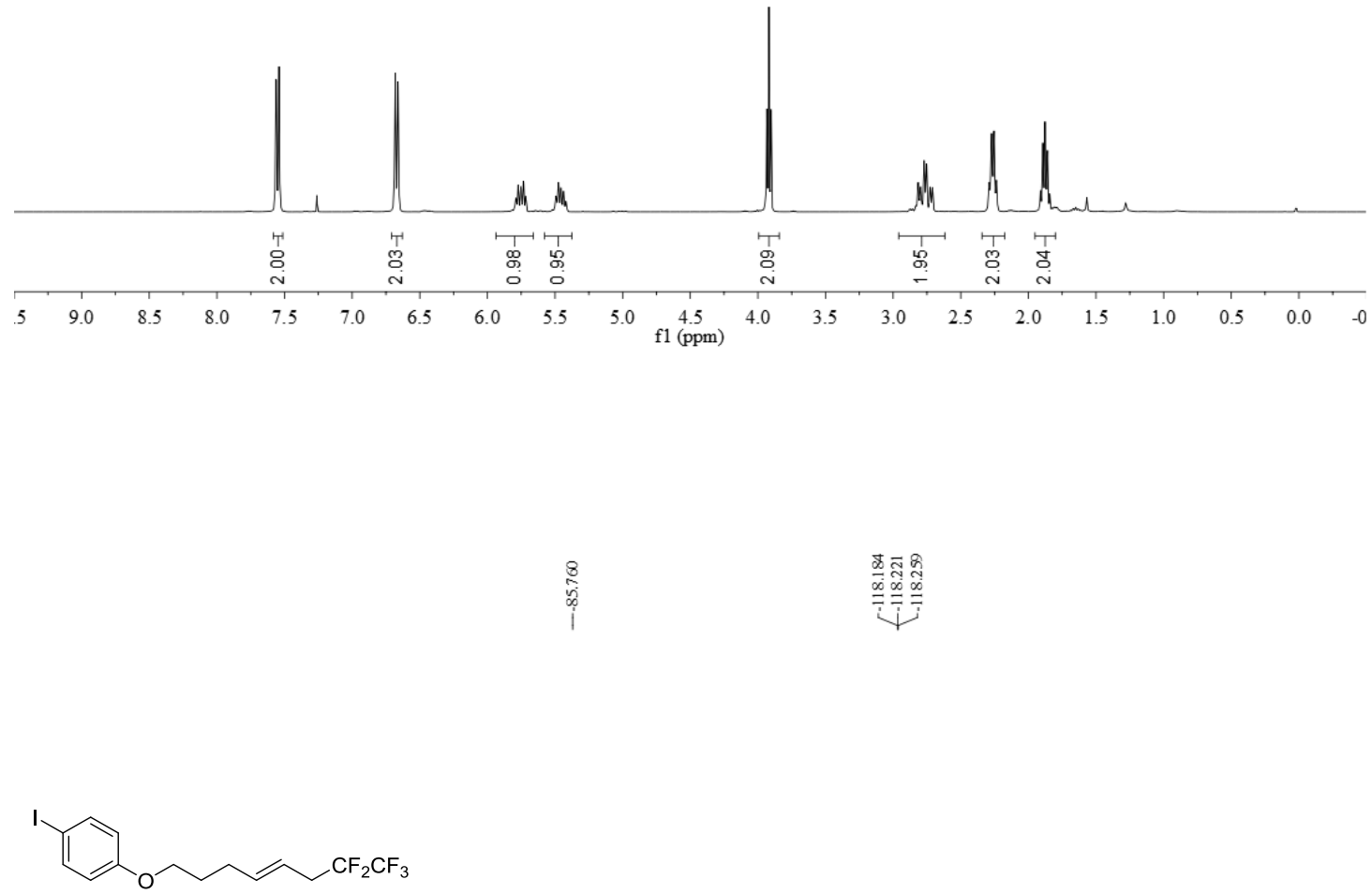

$2 \mathrm{k}\left({ }^{19} \mathrm{~F}\right.$ NMR, $\left.\mathrm{CDCl}_{3}, 470 \mathrm{MHz}\right)$

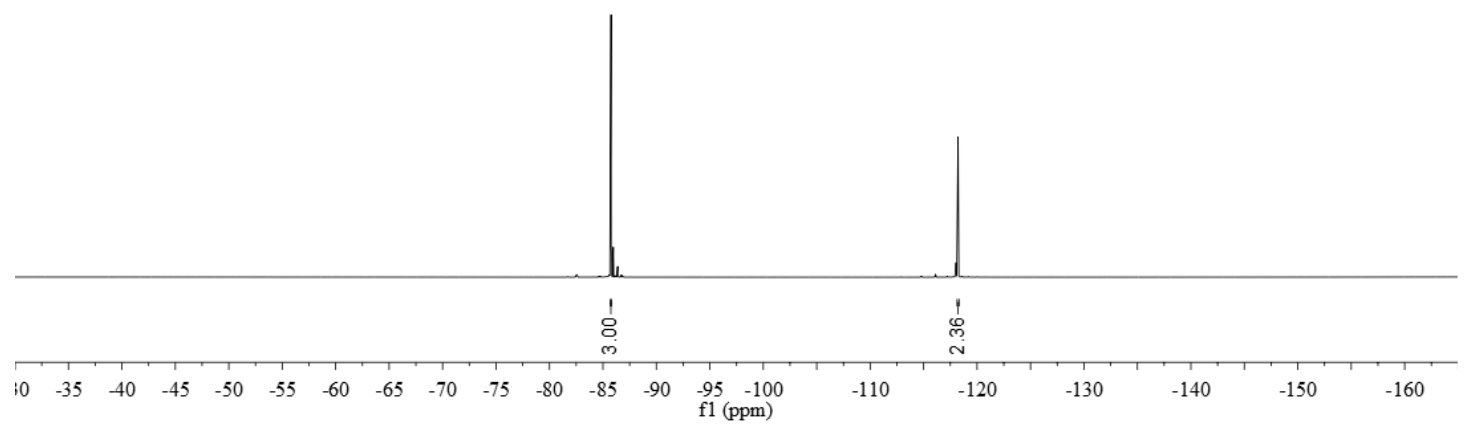




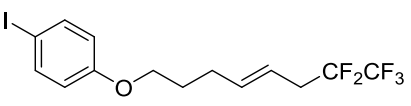

2k ( ${ }^{13} \mathrm{C}$ NMR, $\left.\mathrm{CDCl}_{3}, 101 \mathrm{MHz}\right)$
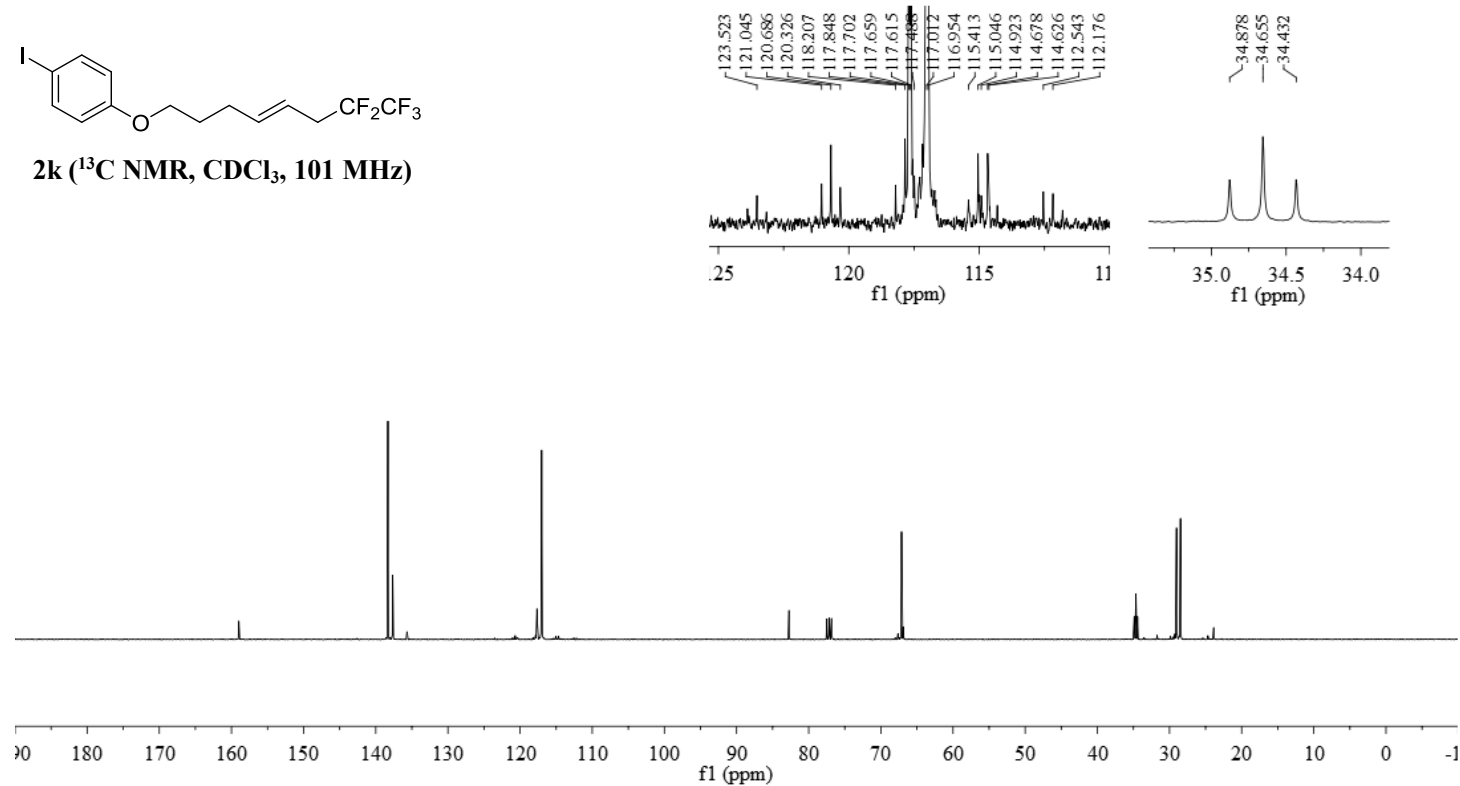

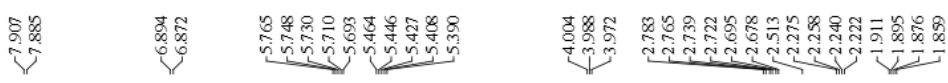

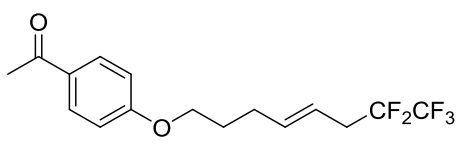

2 I ( ${ }^{1} \mathrm{H}$ NMR, $\left.\mathrm{CDCl}_{3}, 400 \mathrm{MHz}\right)$

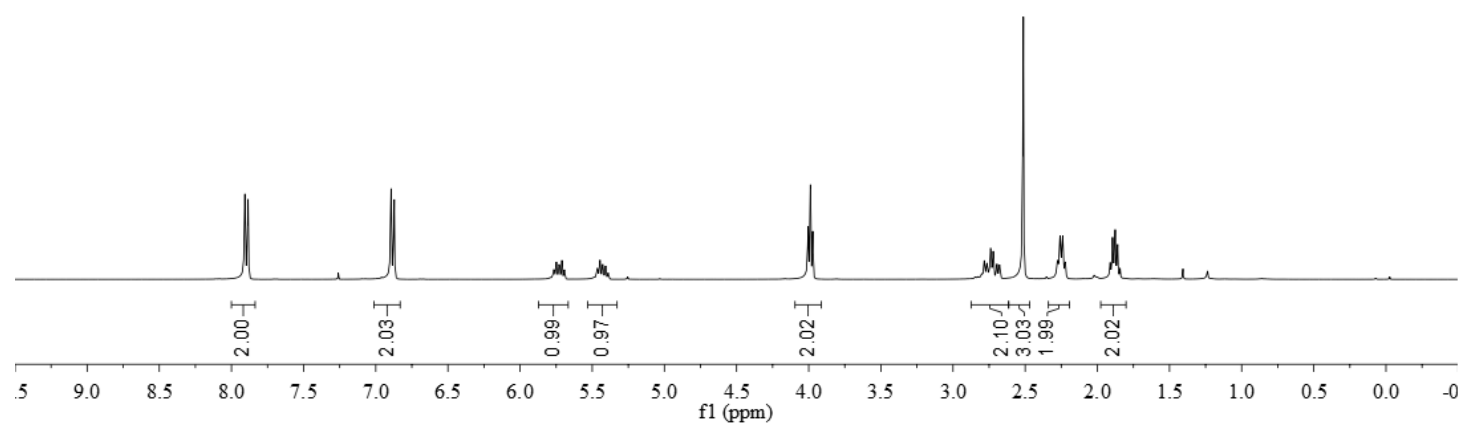




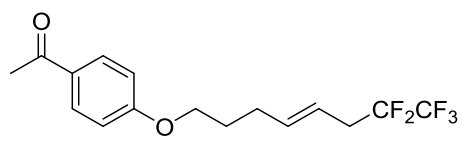

$21\left({ }^{19} \mathrm{~F} \mathrm{NMR,} \mathrm{CDCl}_{3}, 470 \mathrm{MHz}\right)$

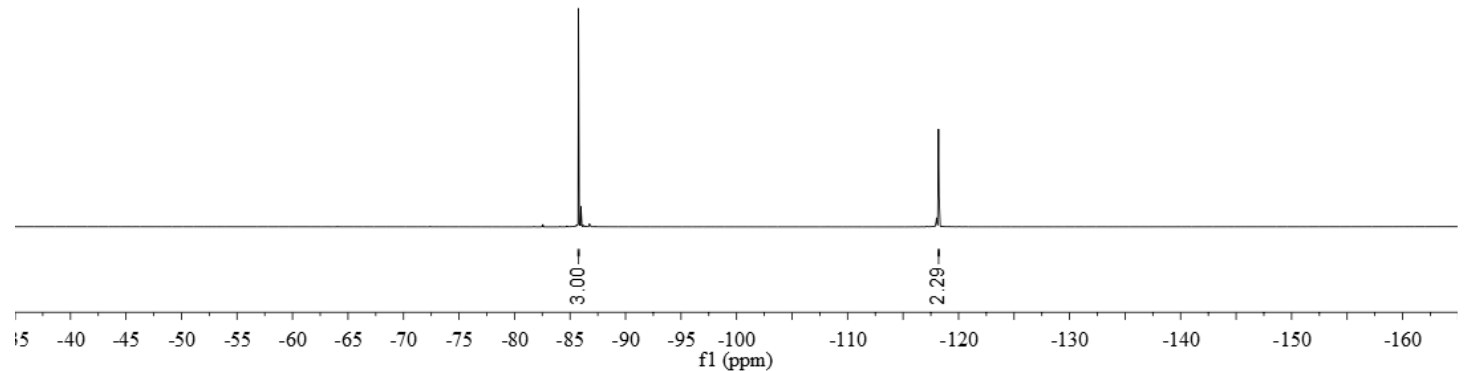

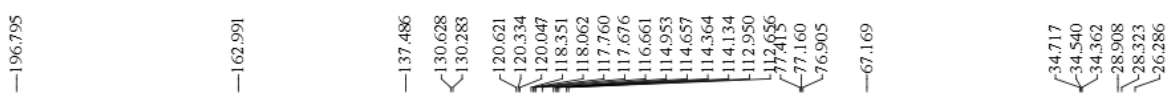
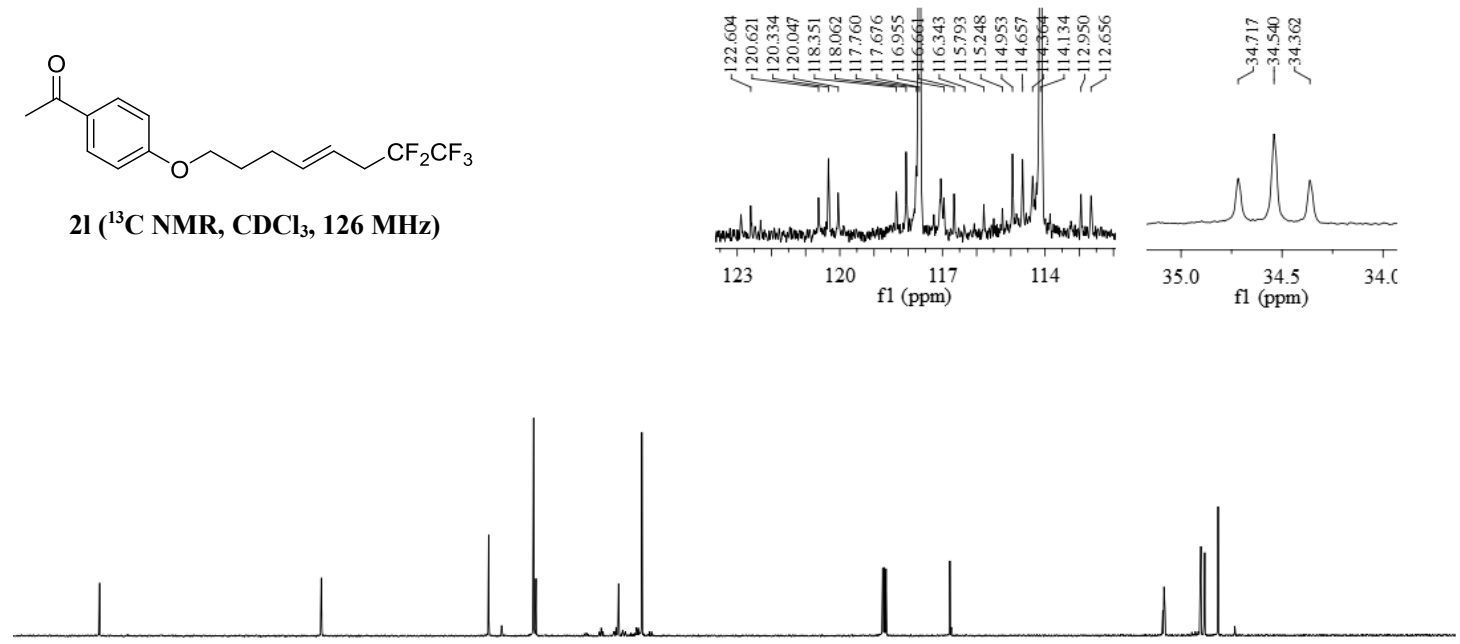

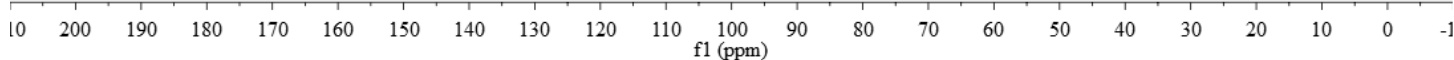



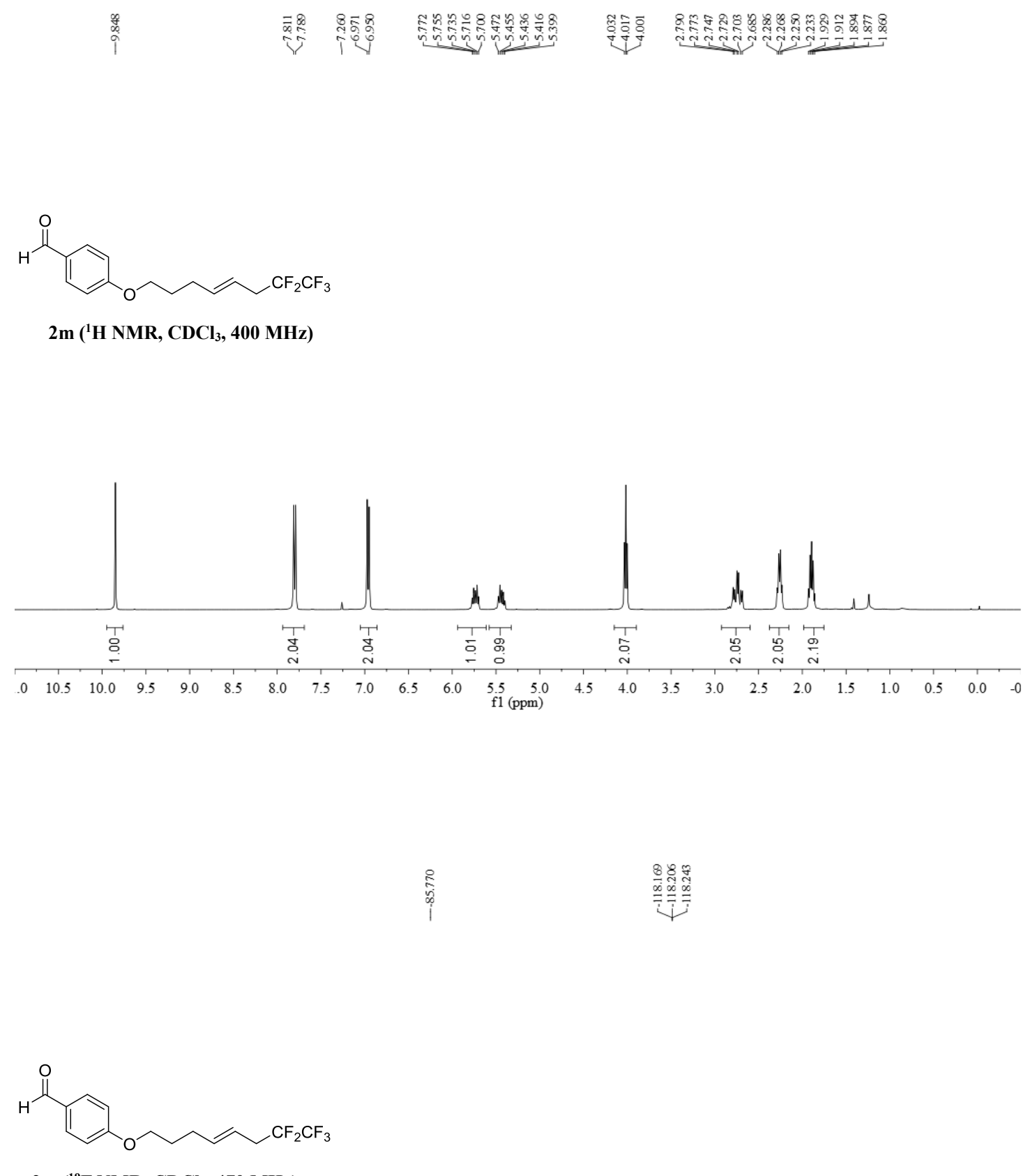

$2 \mathrm{~m}\left({ }^{19} \mathrm{~F}\right.$ NMR, $\left.\mathrm{CDCl}_{3}, 470 \mathrm{MHz}\right)$

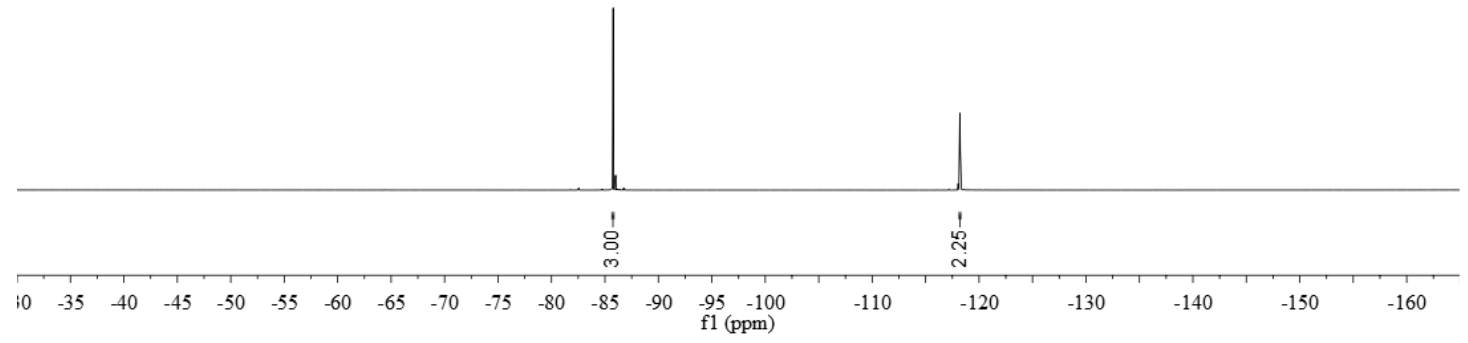




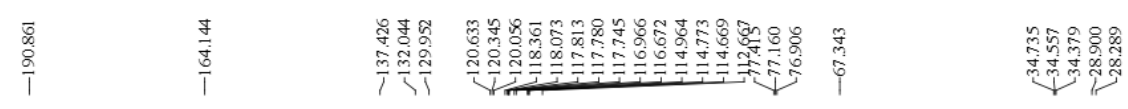

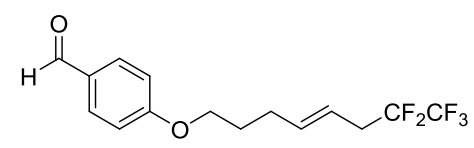

$2 \mathrm{~m}\left({ }^{13} \mathrm{C}\right.$ NMR, $\left.\mathrm{CDCl}_{3}, 126 \mathrm{MHz}\right)$
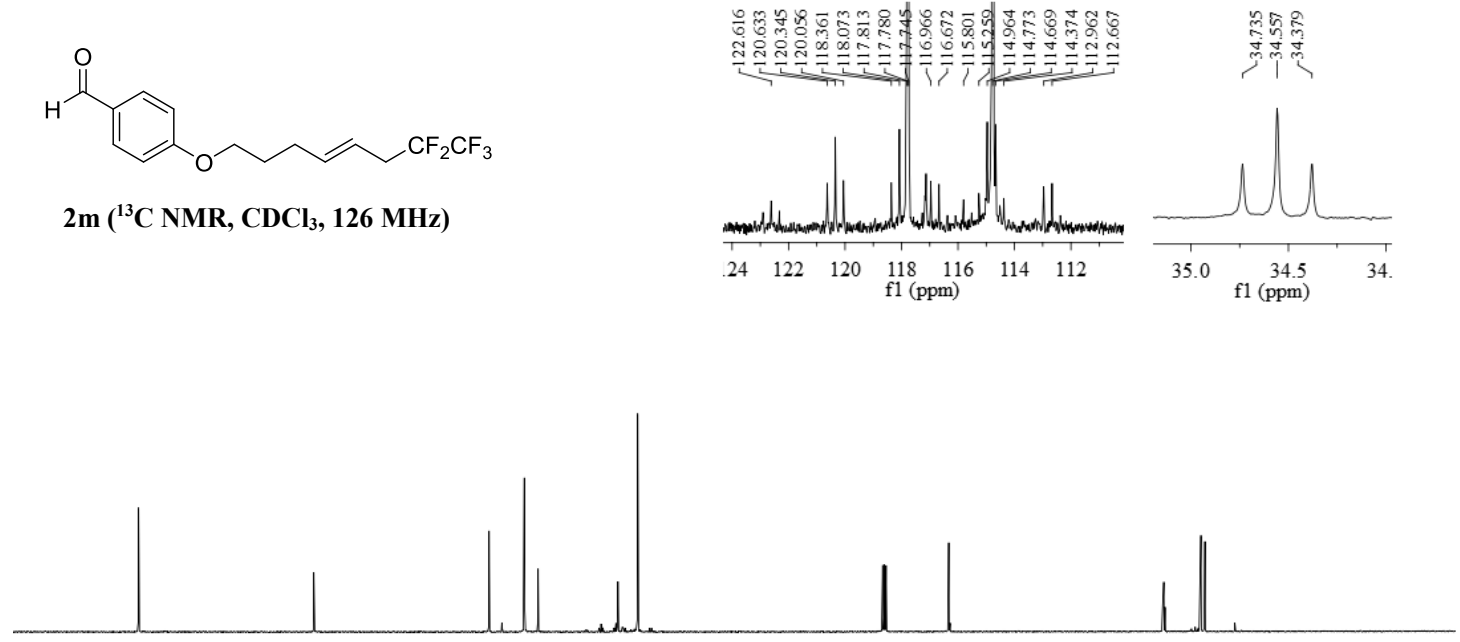

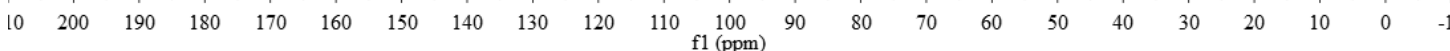

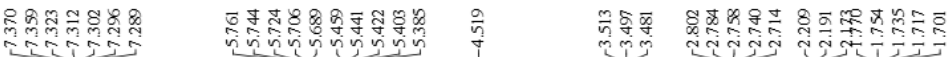

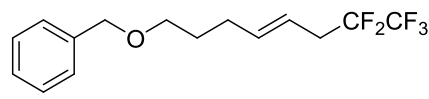

2n ( $\left.{ }^{1} \mathrm{H} \mathrm{NMR}, \mathrm{CDCl}_{3}, 400 \mathrm{MHz}\right)$

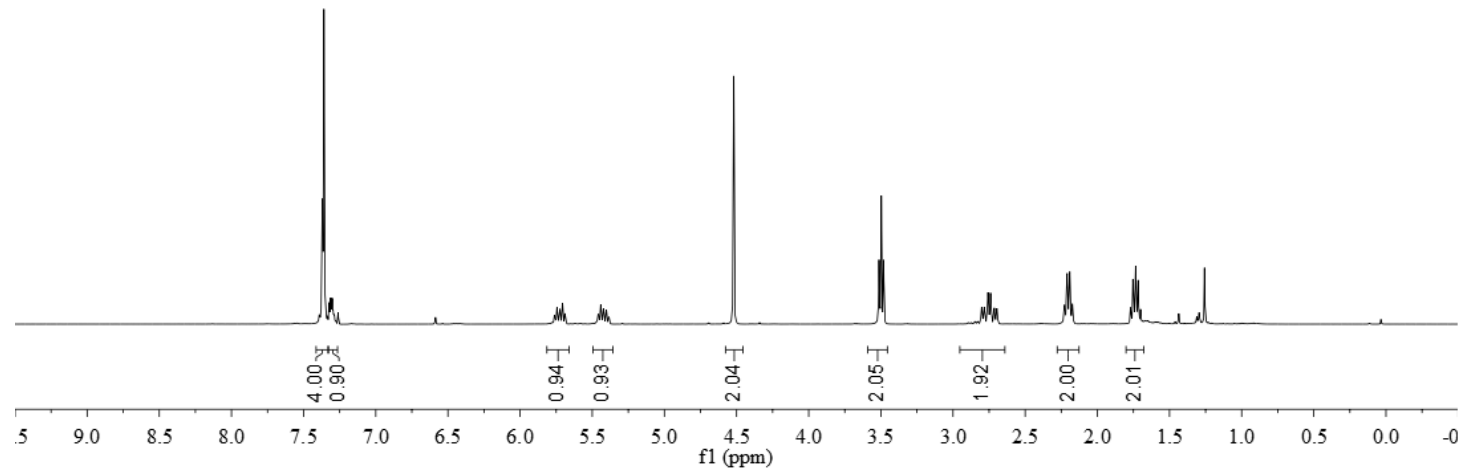



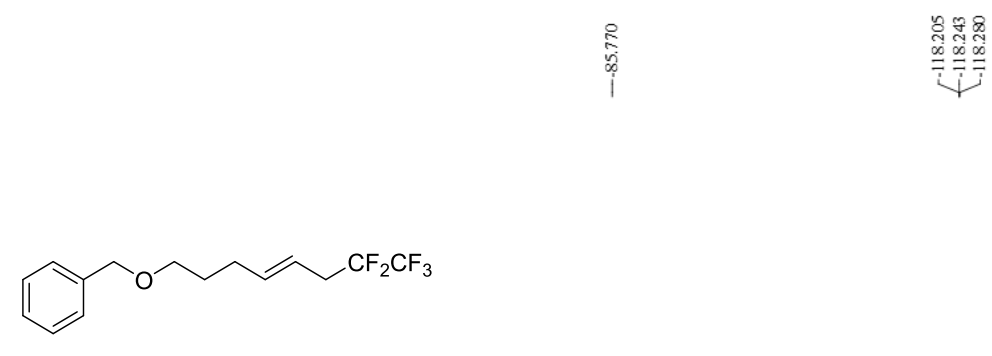

2n $\left({ }^{19} \mathrm{~F} \mathrm{NMR}, \mathrm{CDCl}_{3}, 470 \mathrm{MHz}\right)$

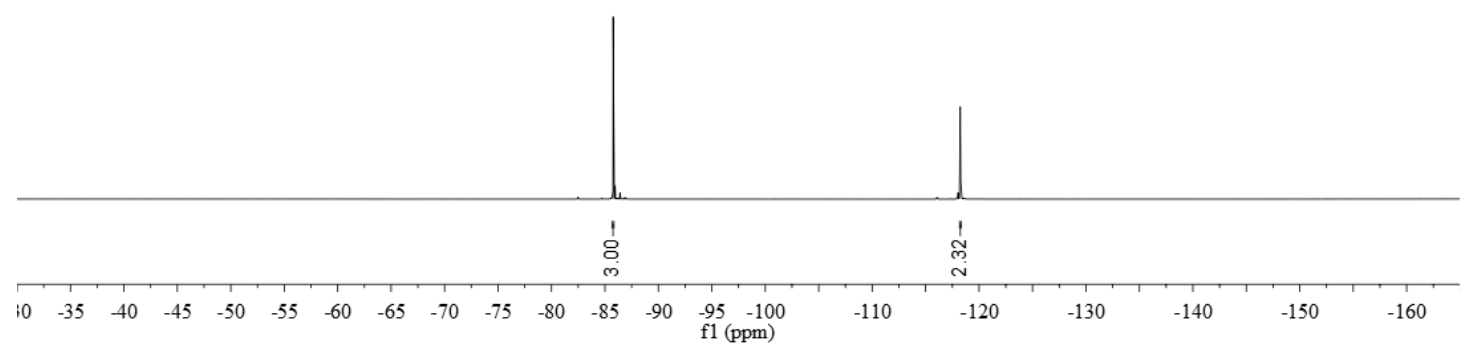

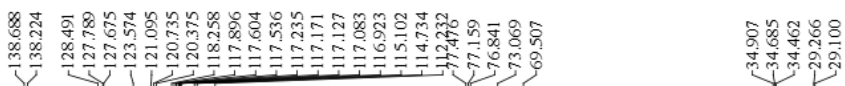
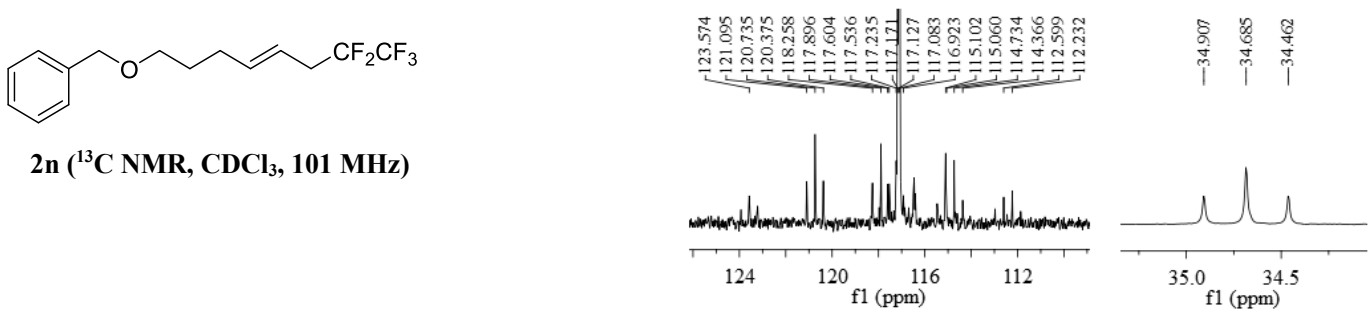

2n $\left({ }^{13} \mathrm{C} \mathrm{NMR,} \mathrm{CDCl}_{3}, 101 \mathrm{MHz}\right)$

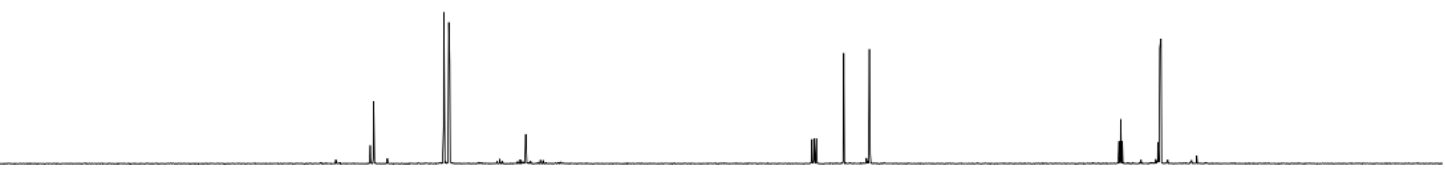

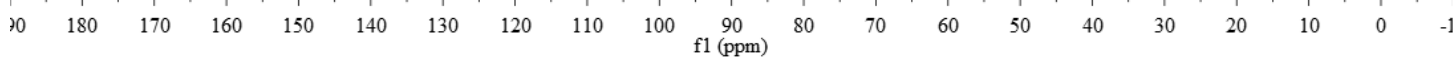



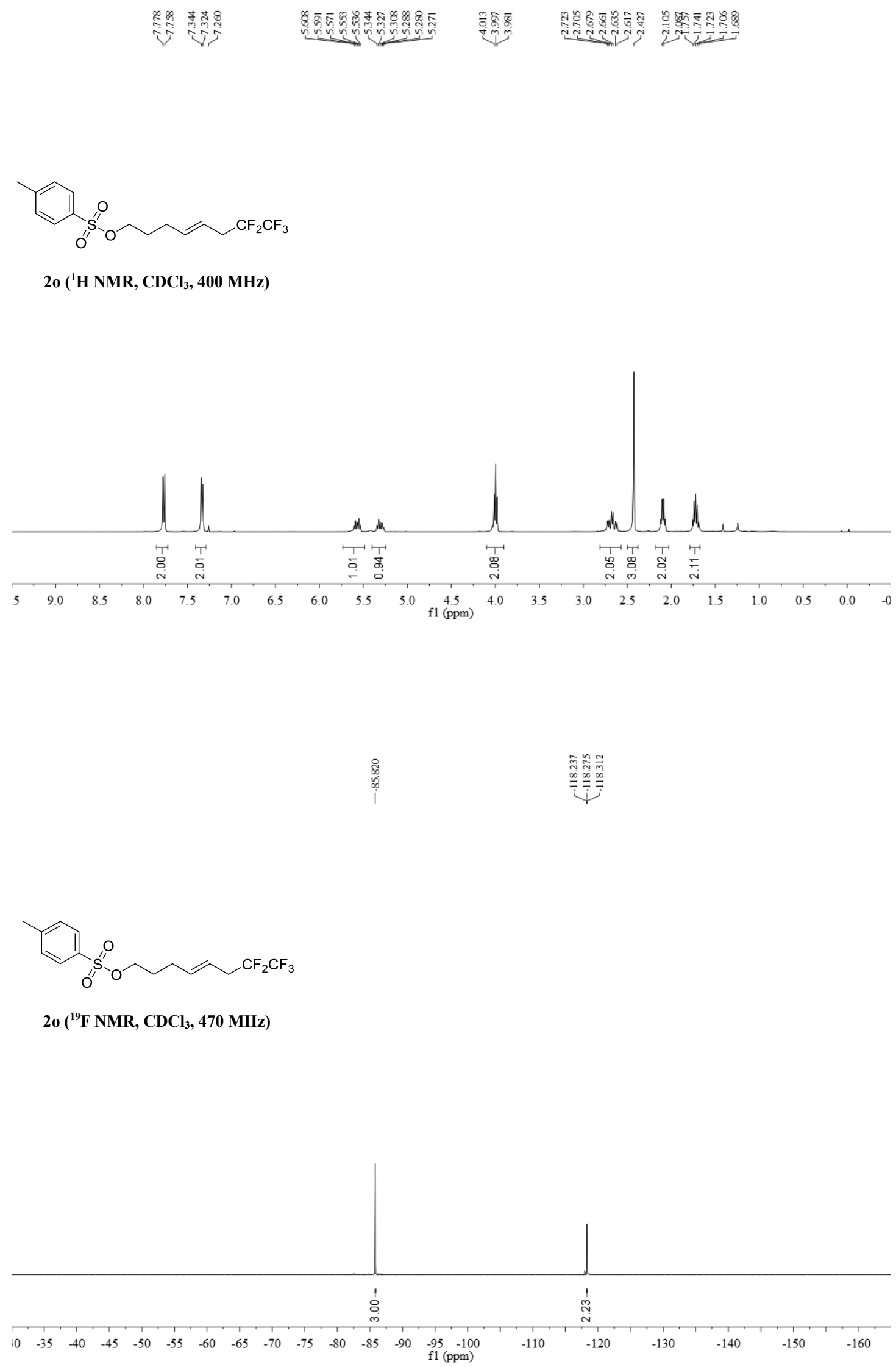


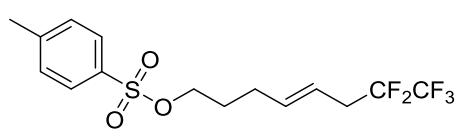

$20\left({ }^{13} \mathrm{C}\right.$ NMR, $\left.\mathrm{CDCl}_{3}, 101 \mathrm{MHz}\right)$

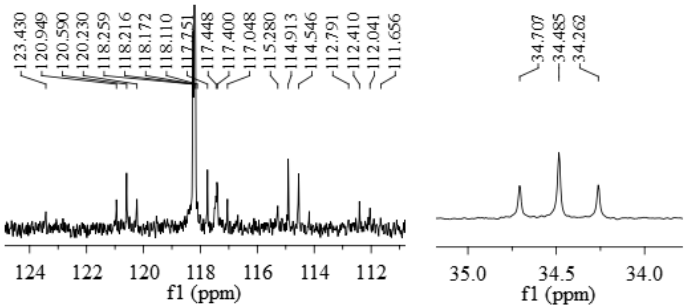

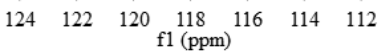
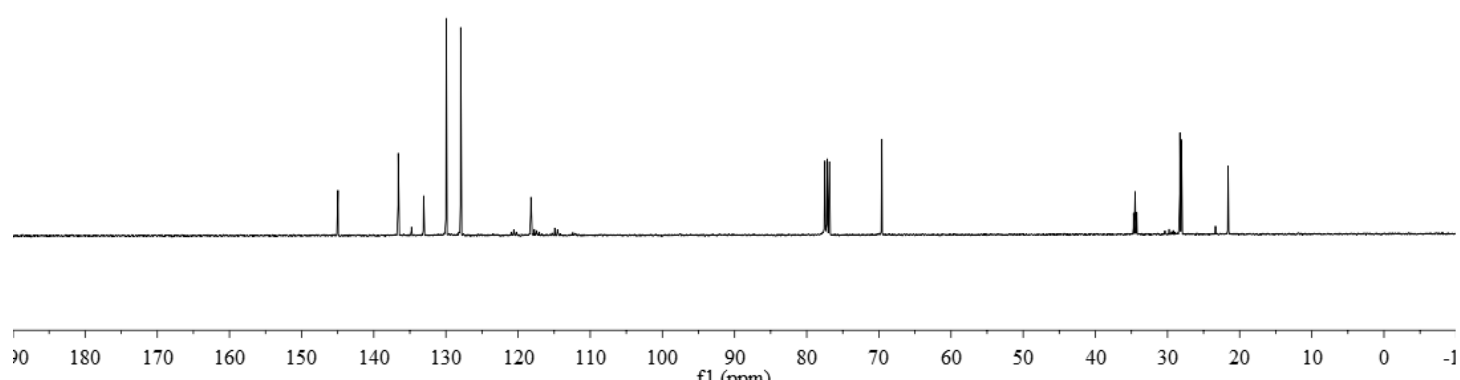

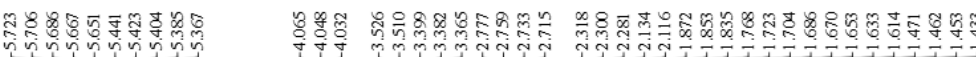

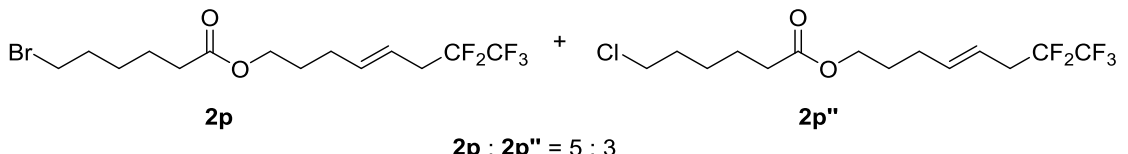

( ${ }^{1} \mathrm{H}$ NMR, $\left.\mathrm{CDCl}_{3}, 400 \mathrm{MHz}\right)$

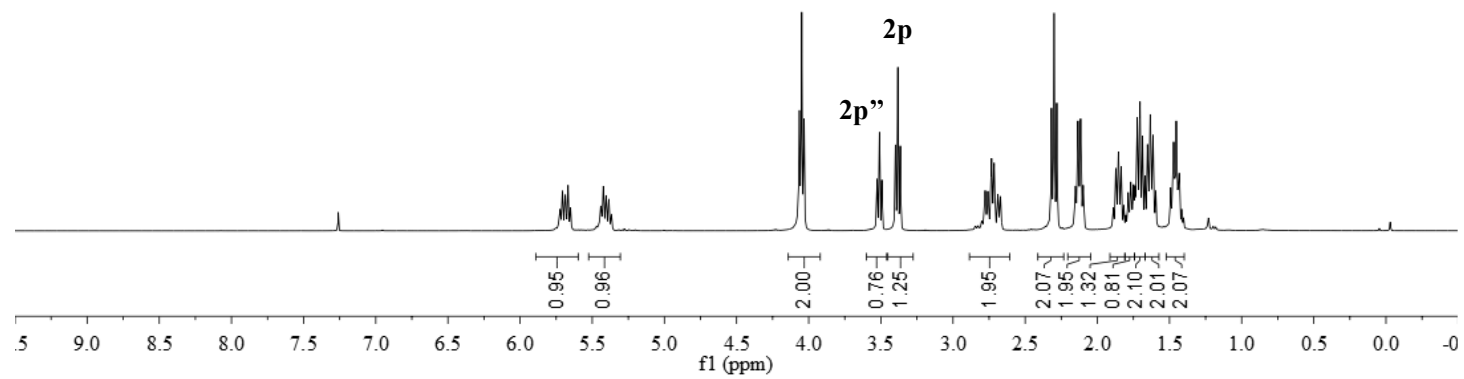




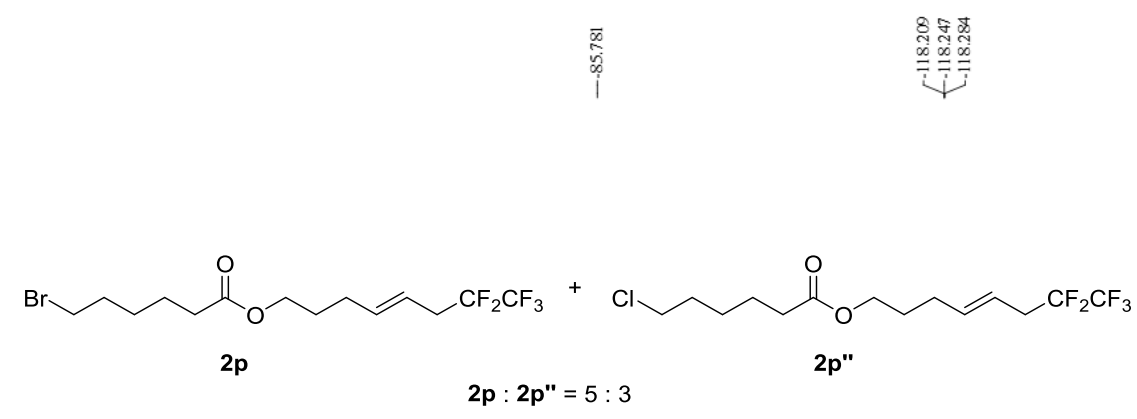

$\left({ }^{19} \mathrm{~F} \mathrm{NMR}, \mathrm{CDCl}_{3}, 470 \mathrm{MHz}\right)$
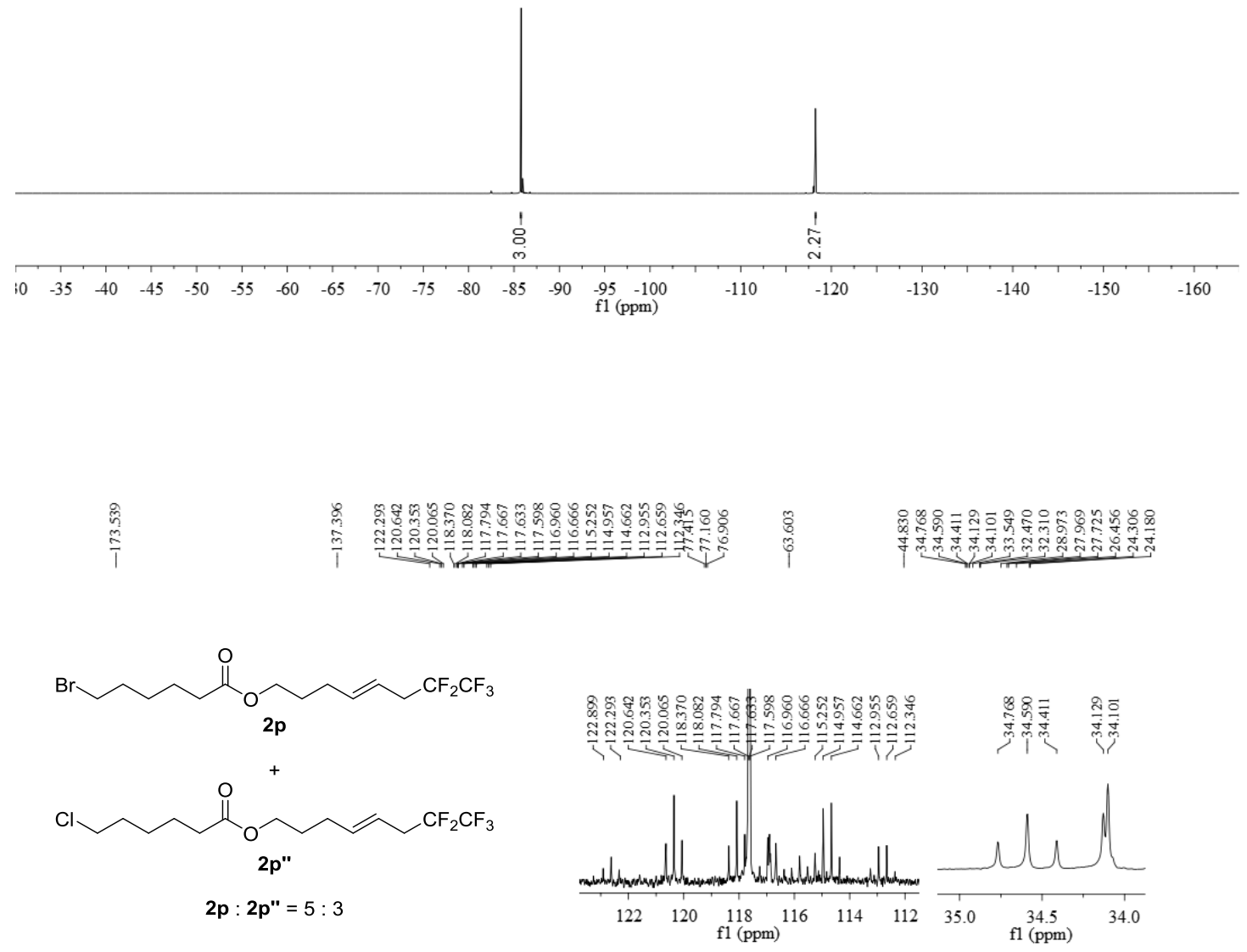

$\left({ }^{13} \mathrm{C}\right.$ NMR, $\left.\mathrm{CDCl}_{3}, 126 \mathrm{MHz}\right)$

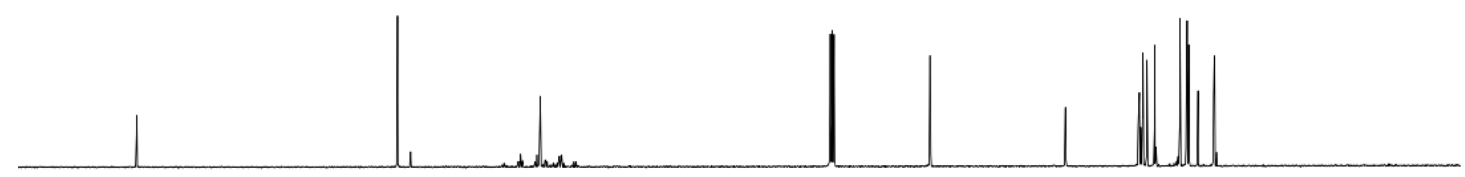

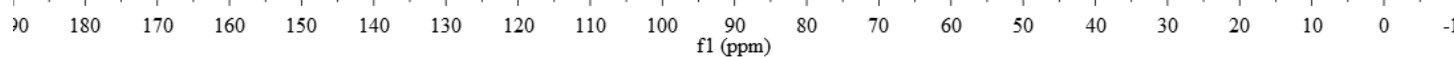



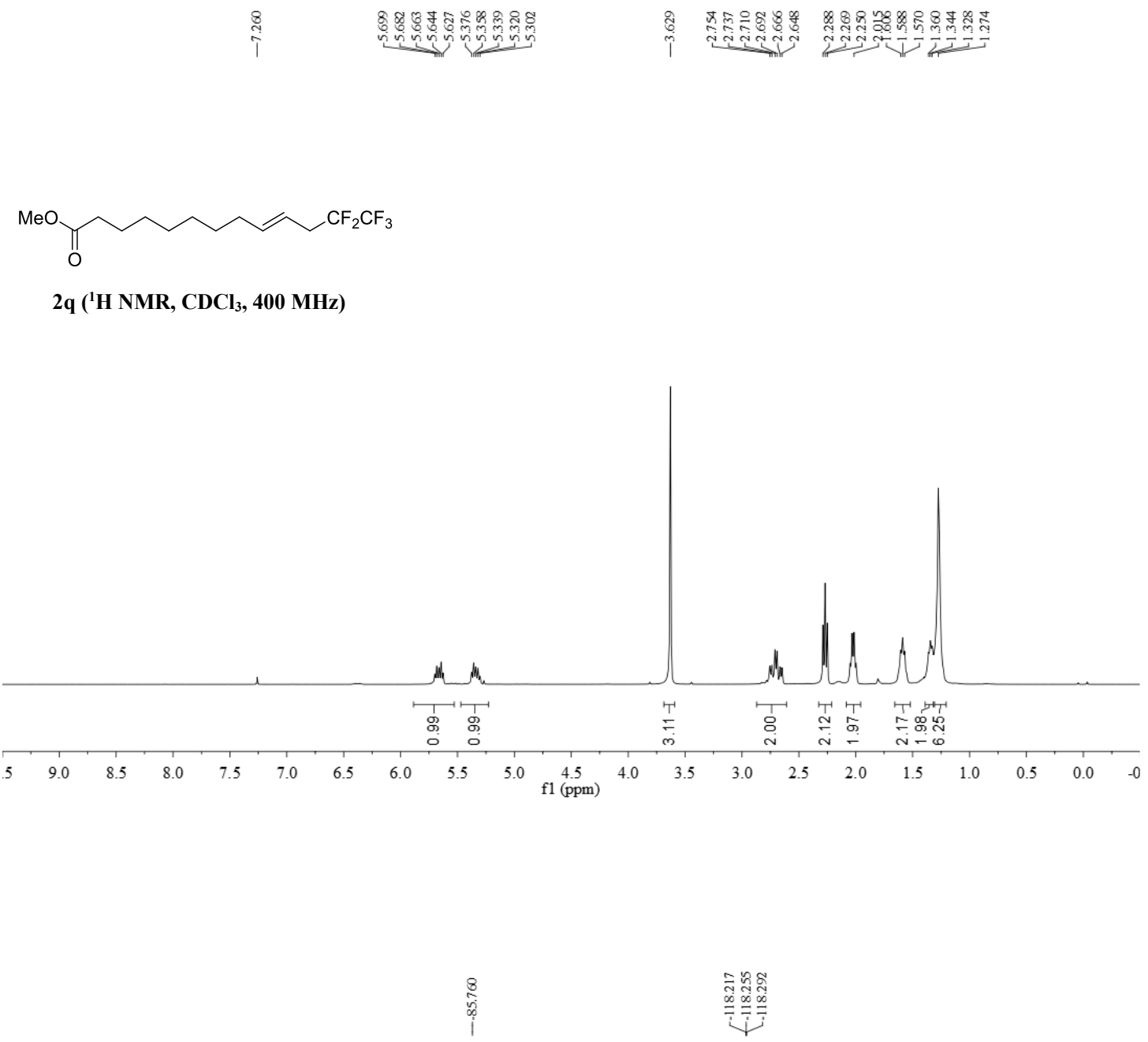

${ }_{\mathrm{O}}^{\mathrm{MeO}} \mathrm{CF}_{2} \mathrm{CF}_{3}$

$2 q\left({ }^{19} \mathrm{~F}\right.$ NMR, $\left.\mathrm{CDCl}_{3}, 470 \mathrm{MHz}\right)$

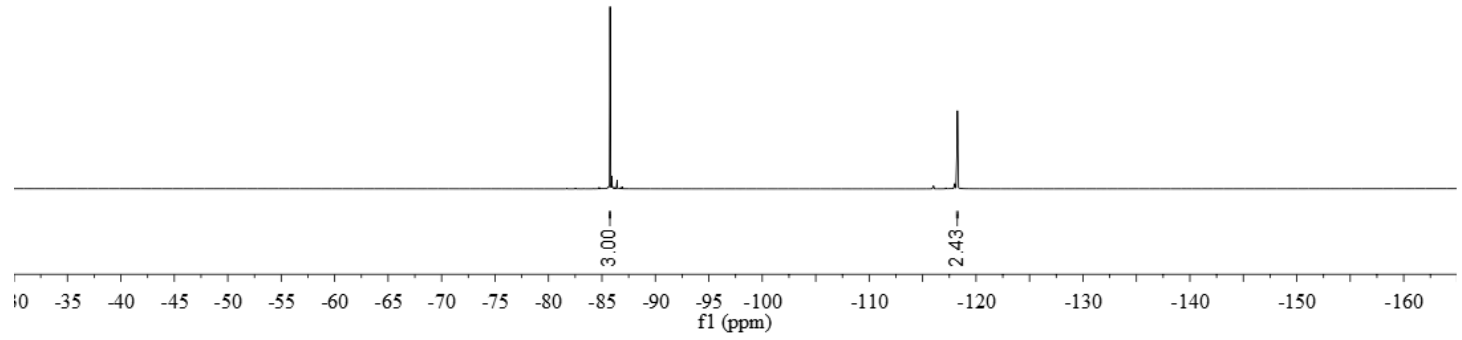




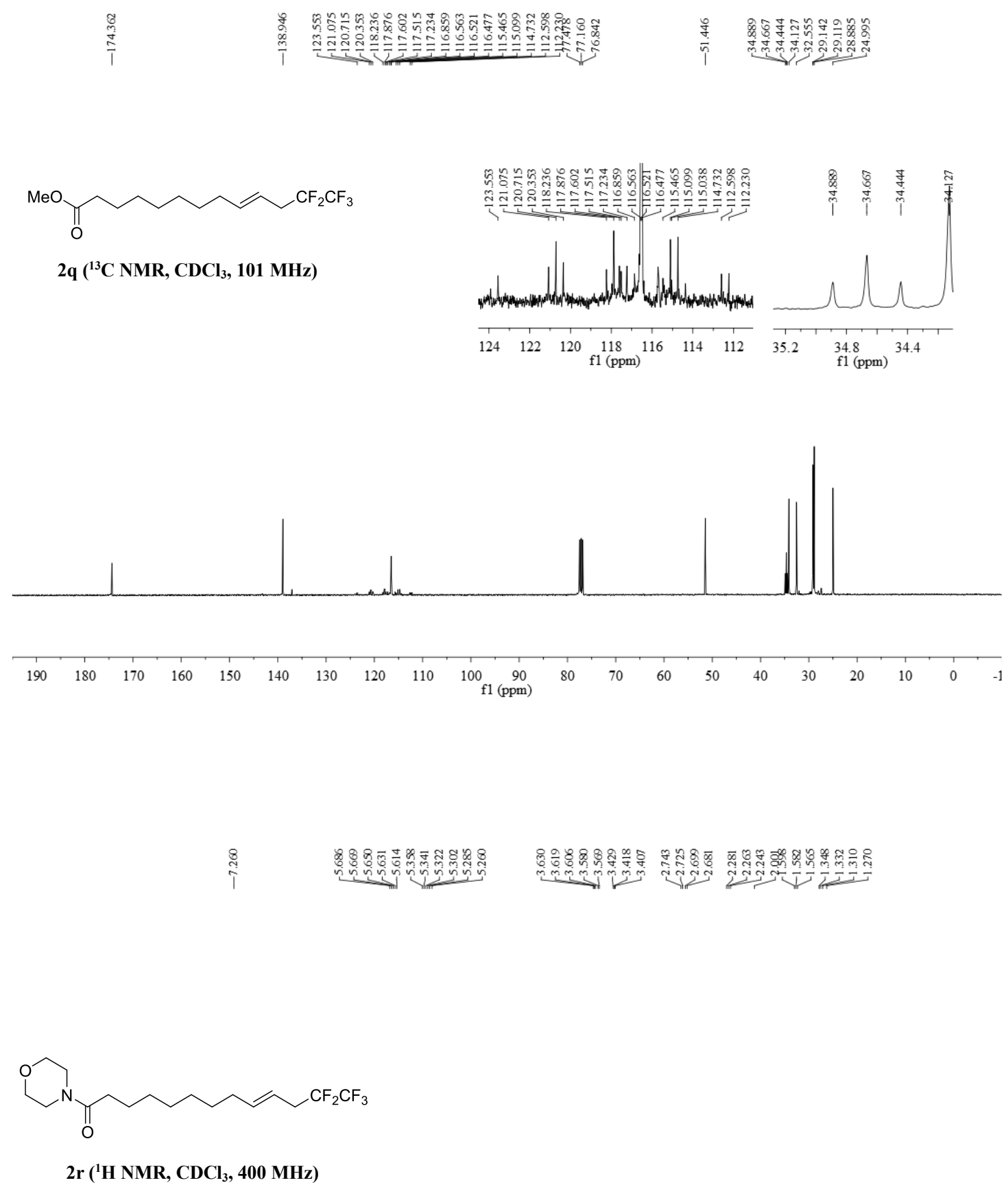

2r $\left({ }^{1} \mathrm{H}\right.$ NMR, $\left.\mathrm{CDCl}_{3}, 400 \mathrm{MHz}\right)$

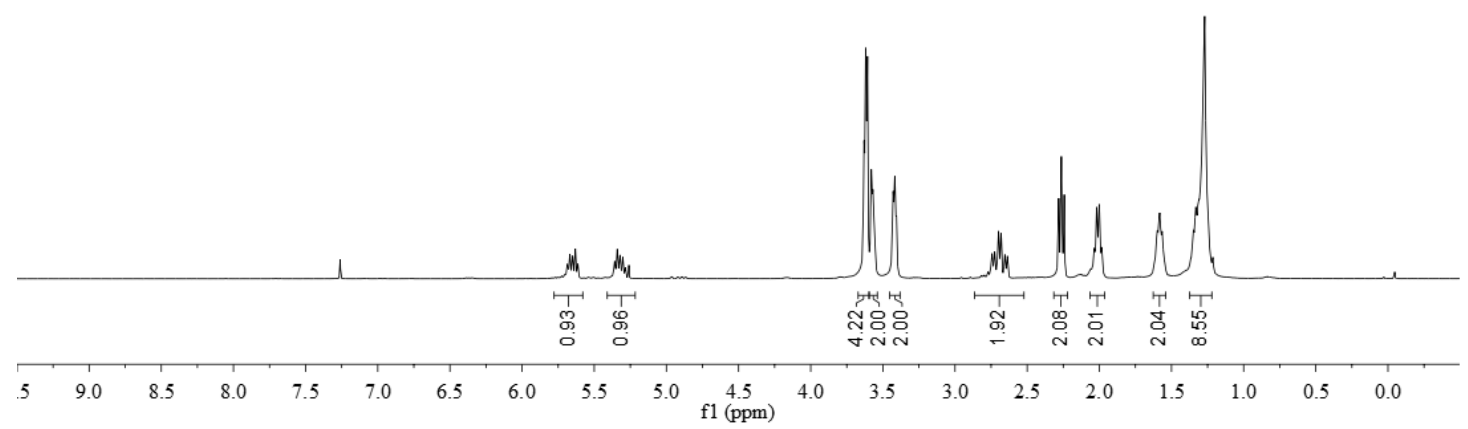



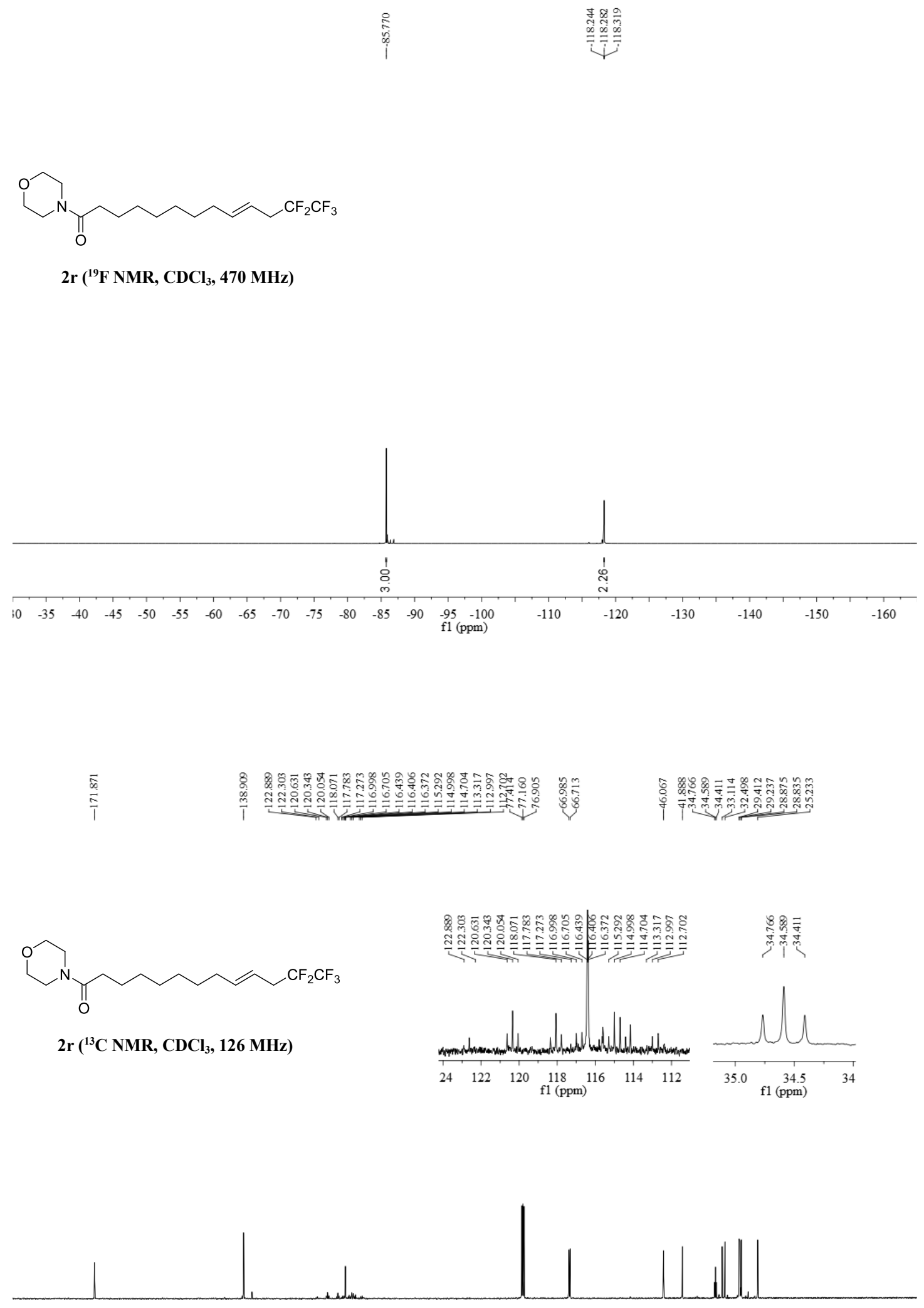

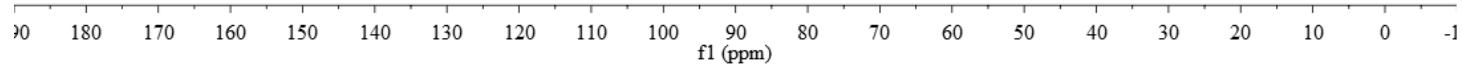


$\sim \mathrm{CF}_{2} \mathrm{CF}_{3}$

2s ( ${ }^{1} \mathrm{H}$ NMR, $\left.\mathrm{CDCl}_{3}, 400 \mathrm{MHz}\right)$
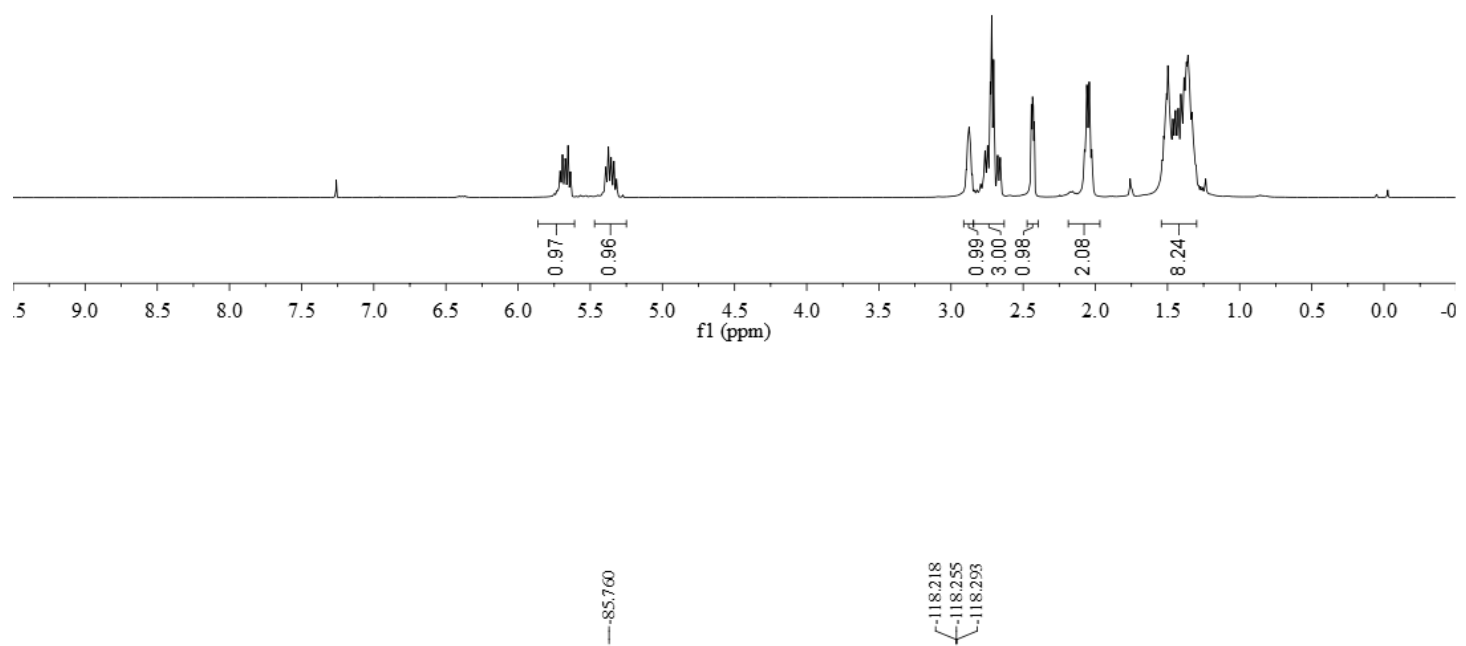

$\sim \mathrm{CF}_{2} \mathrm{CF}_{3}$

2s ( ${ }^{19} \mathrm{~F}$ NMR, $\left.\mathrm{CDCl}_{3}, 470 \mathrm{MHz}\right)$

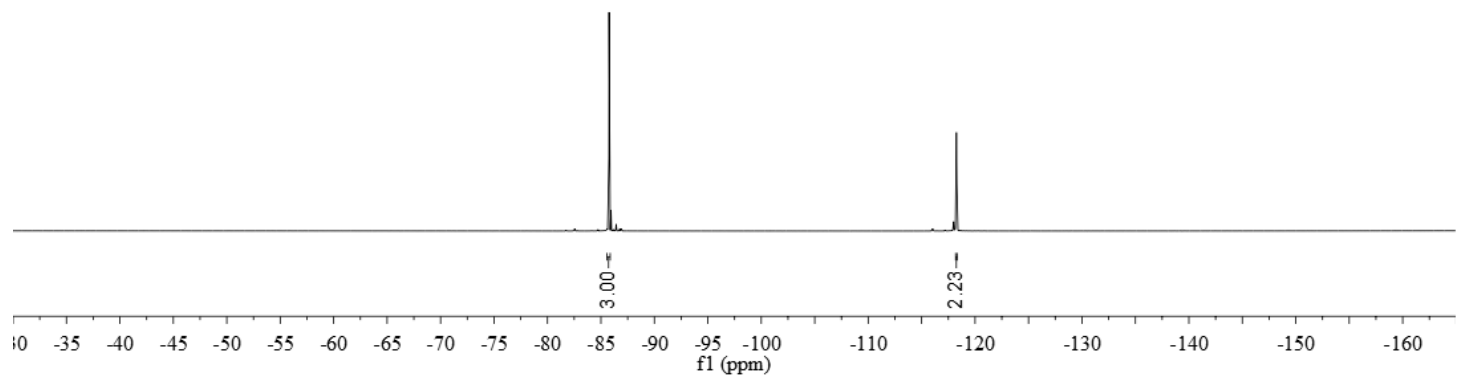




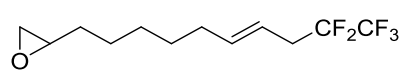

2s $\left({ }^{13} \mathrm{C}\right.$ NMR, $\left.\mathrm{CDCl}_{3}, 101 \mathrm{MHz}\right)$
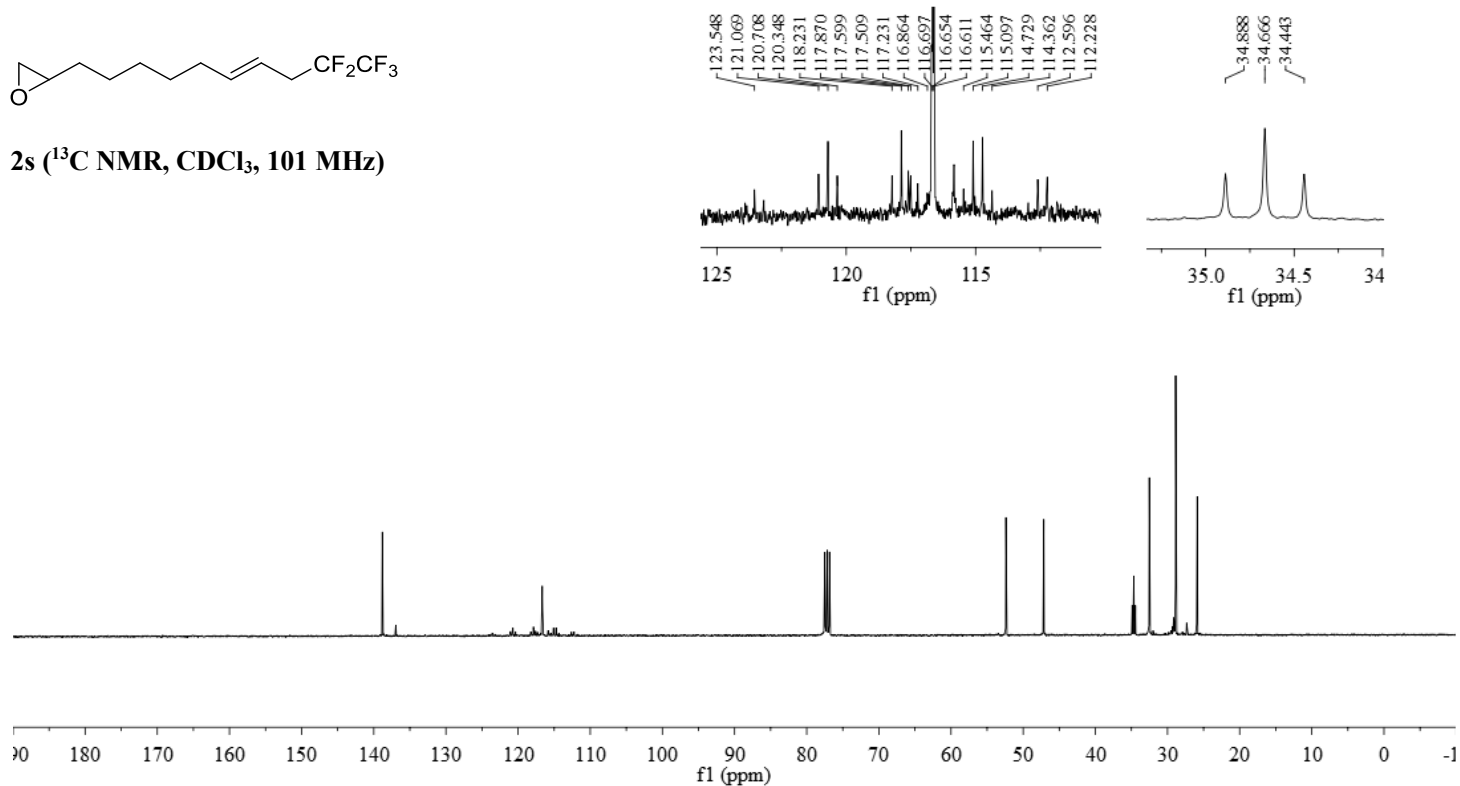

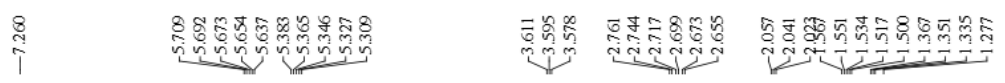

$\mathrm{CF}_{2} \mathrm{CF}_{3}$

2t ( ${ }^{1} \mathrm{H}$ NMR, $\left.\mathrm{CDCl}_{3}, 400 \mathrm{MHz}\right)$

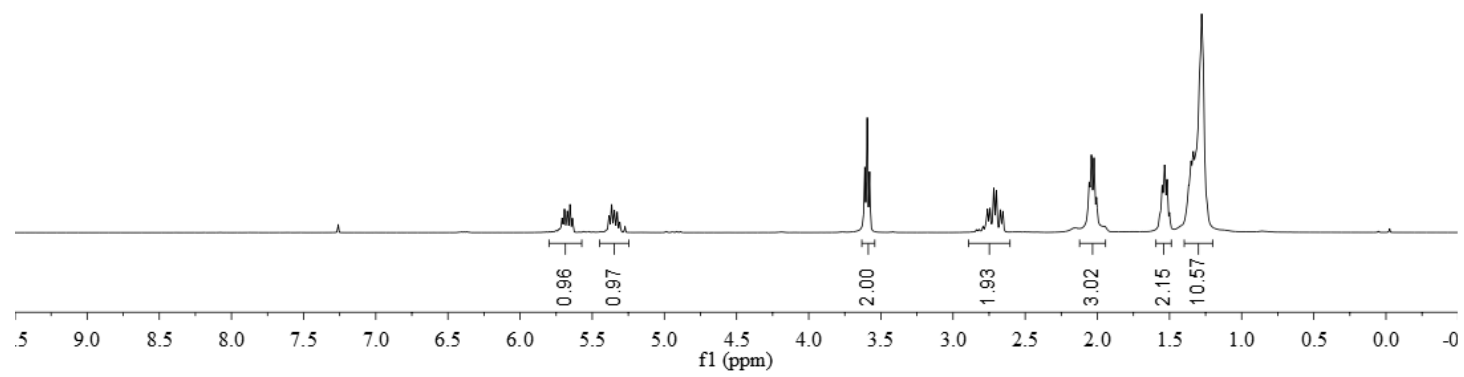




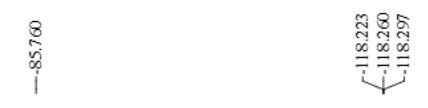

$\mathrm{CF}_{2} \mathrm{CF}_{3}$

2t ( $\left.{ }^{19} \mathrm{~F} \mathrm{NMR}, \mathrm{CDCl}_{3}, 470 \mathrm{MHz}\right)$

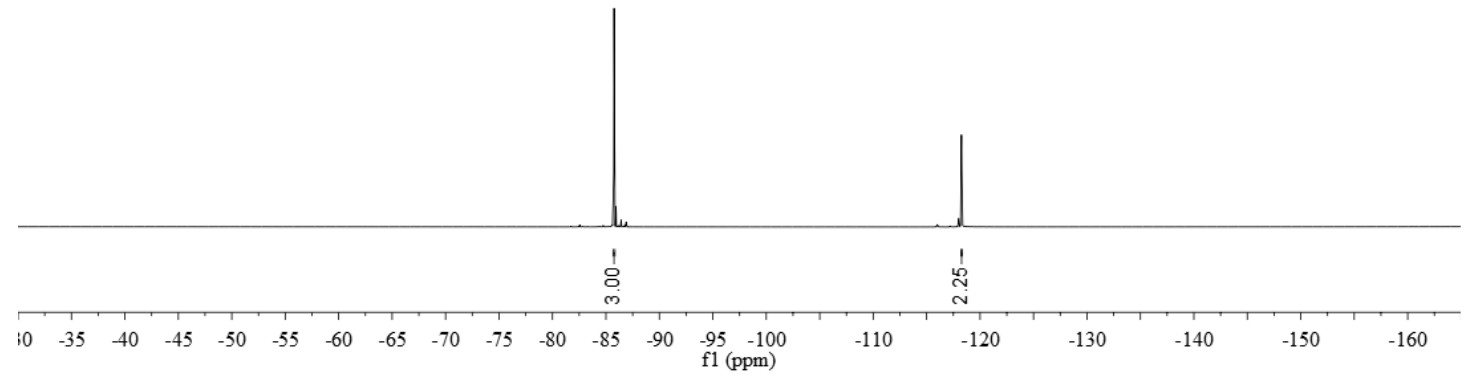

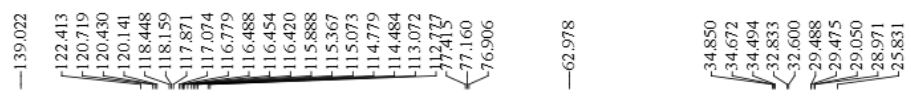

$\mathrm{CPF}_{\mathrm{CF}_{3}}$

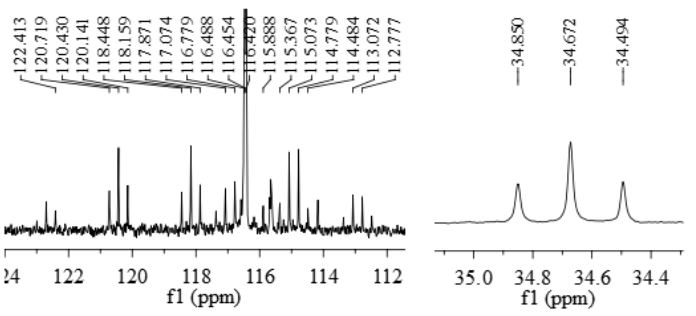

2t $\left({ }^{13} \mathrm{C}\right.$ NMR, $\left.\mathrm{CDCl}_{3}, 126 \mathrm{MHz}\right)$

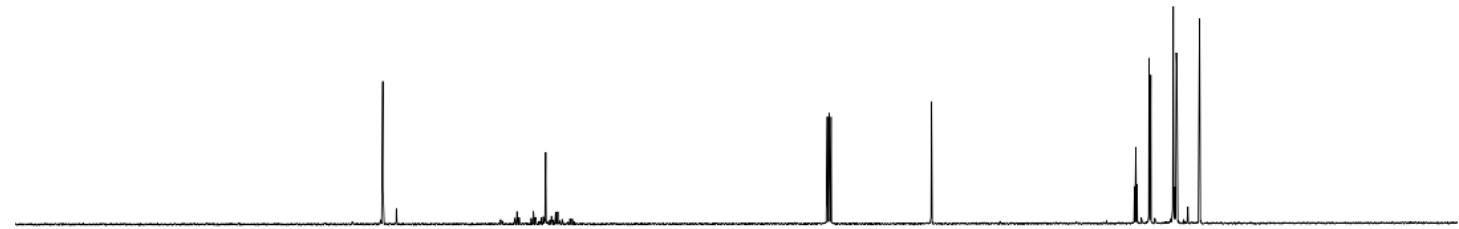

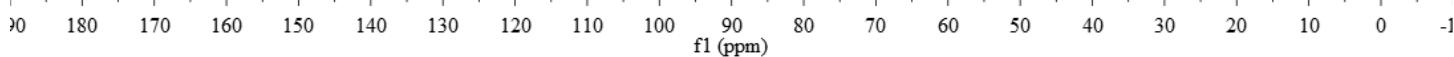



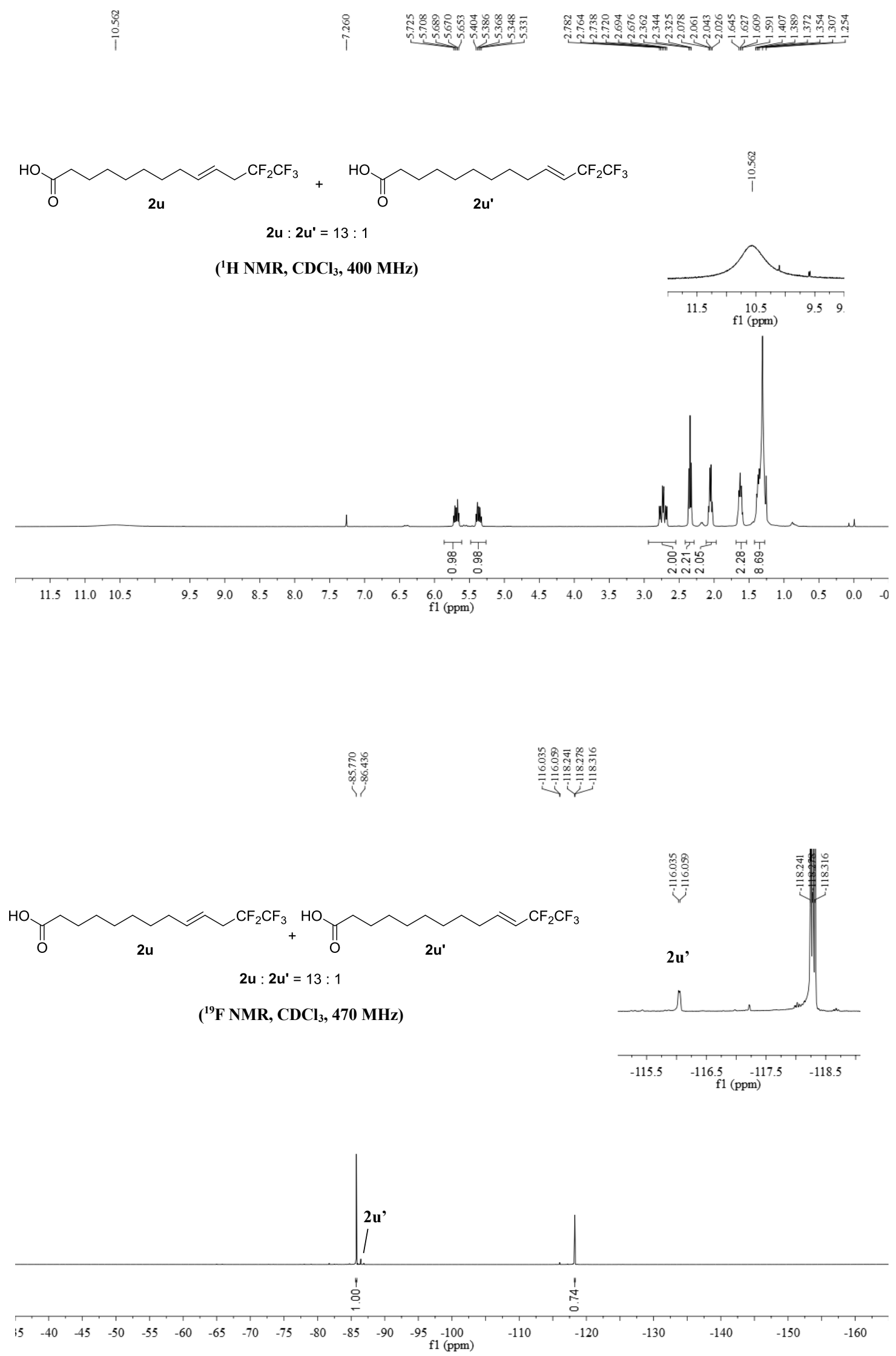


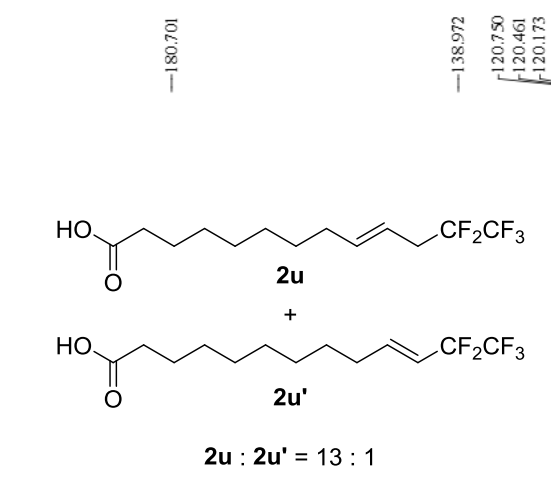

$\left({ }^{13} \mathrm{C}\right.$ NMR, $\left.\mathrm{CDCl}_{3}, 126 \mathrm{MHz}\right)$

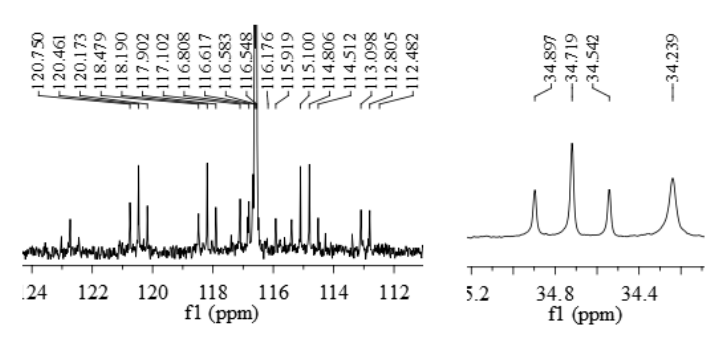

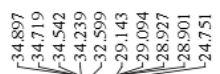
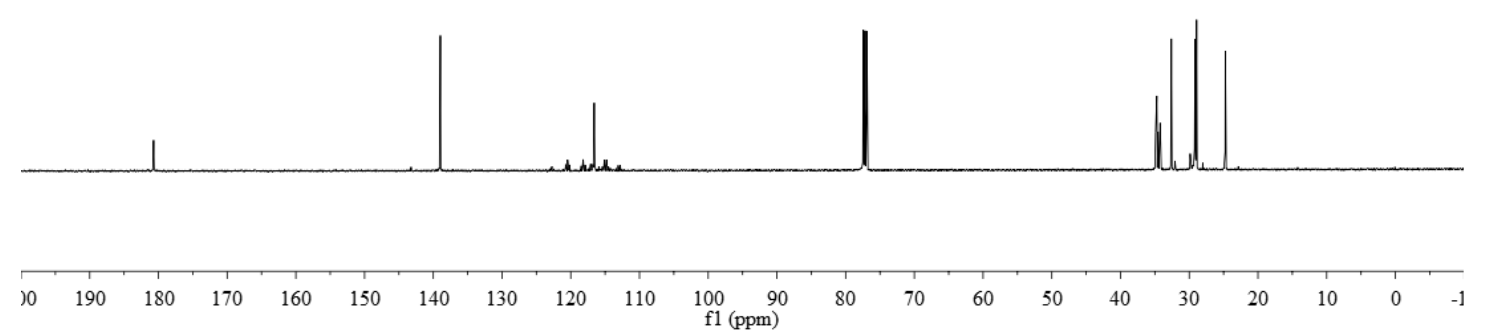

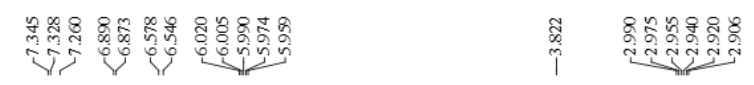<smiles>[C+]#CCCC=Cc1ccc(OC)cc1</smiles>

2v ('H NMR, $\left.\mathrm{CDCl}_{3}, 500 \mathrm{MHz}\right)$

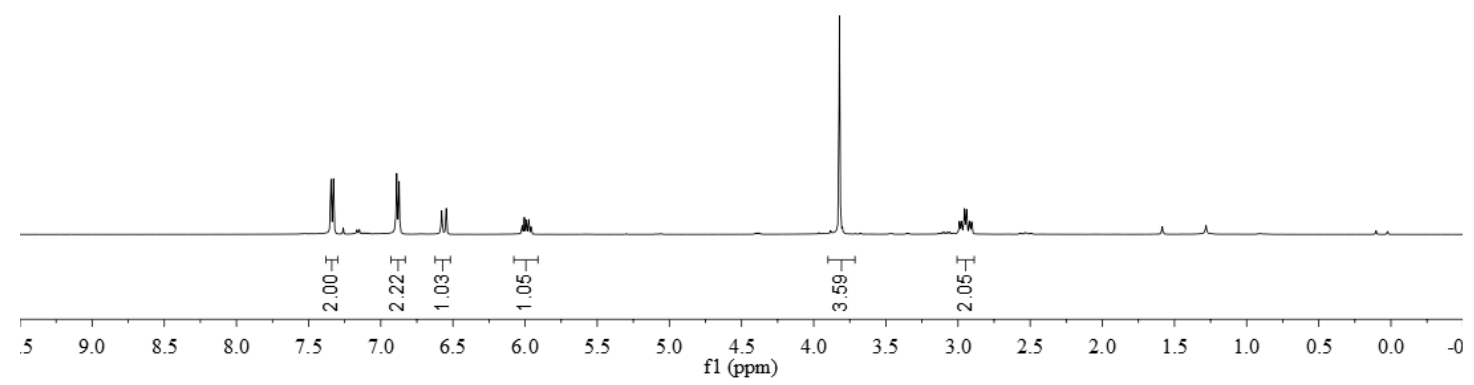




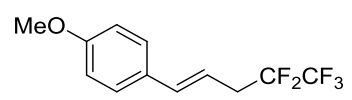

2v $\left({ }^{19} \mathrm{~F}\right.$ NMR, $\left.\mathrm{CDCl}_{3}, 470 \mathrm{MHz}\right)$

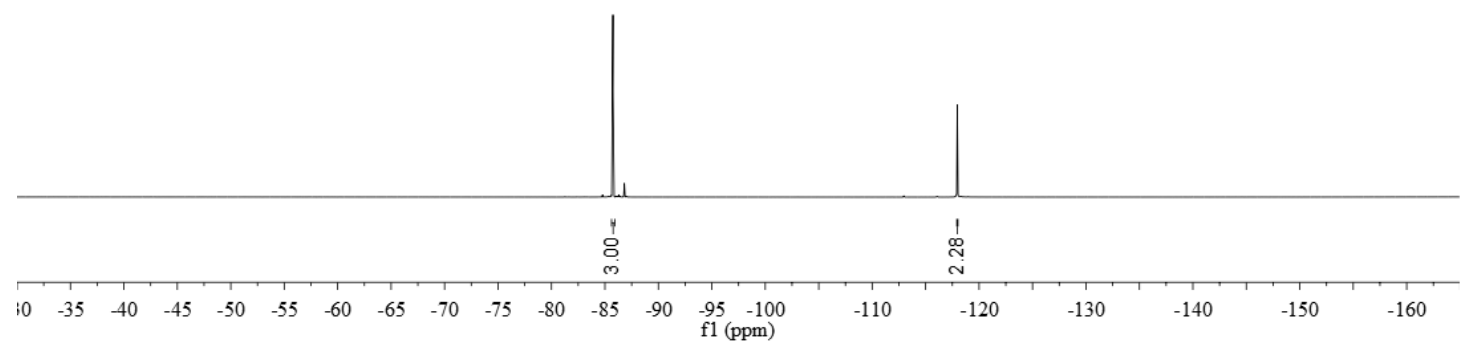

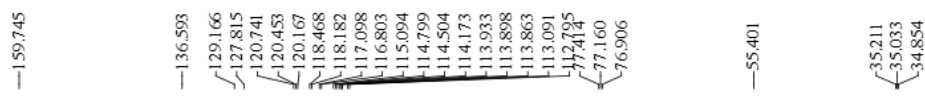

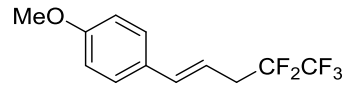

2v $\left({ }^{13} \mathrm{C} N M R, \mathrm{CDCl}_{3}, 126 \mathrm{MHz}\right)$

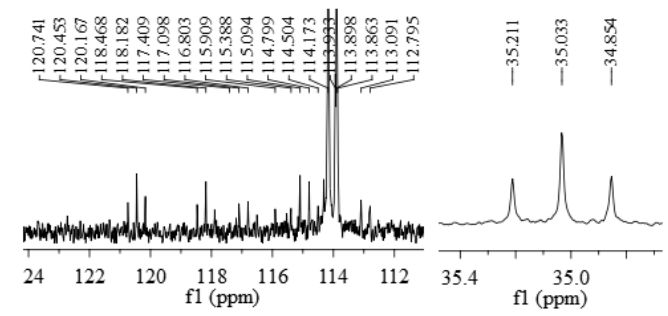

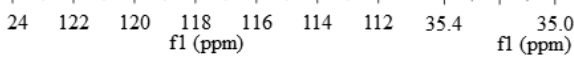

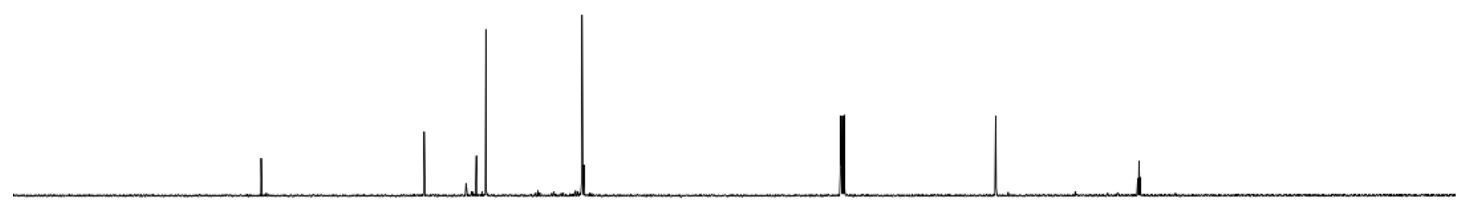

$\begin{array}{lllllllllllllllllllllllll}190 & 180 & 170 & 160 & 150 & 140 & 130 & 120 & 110 & 100 & 90 & 80 & 70 & 60 & 50 & 40 & 30 & 20 & 10 & 0 & -1\end{array}$ 


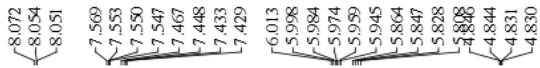

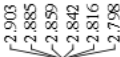

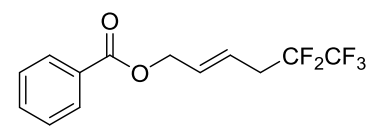

2w ( ${ }^{1} \mathrm{H}$ NMR, $\left.\mathrm{CDCl}_{3}, 400 \mathrm{MHz}\right)$
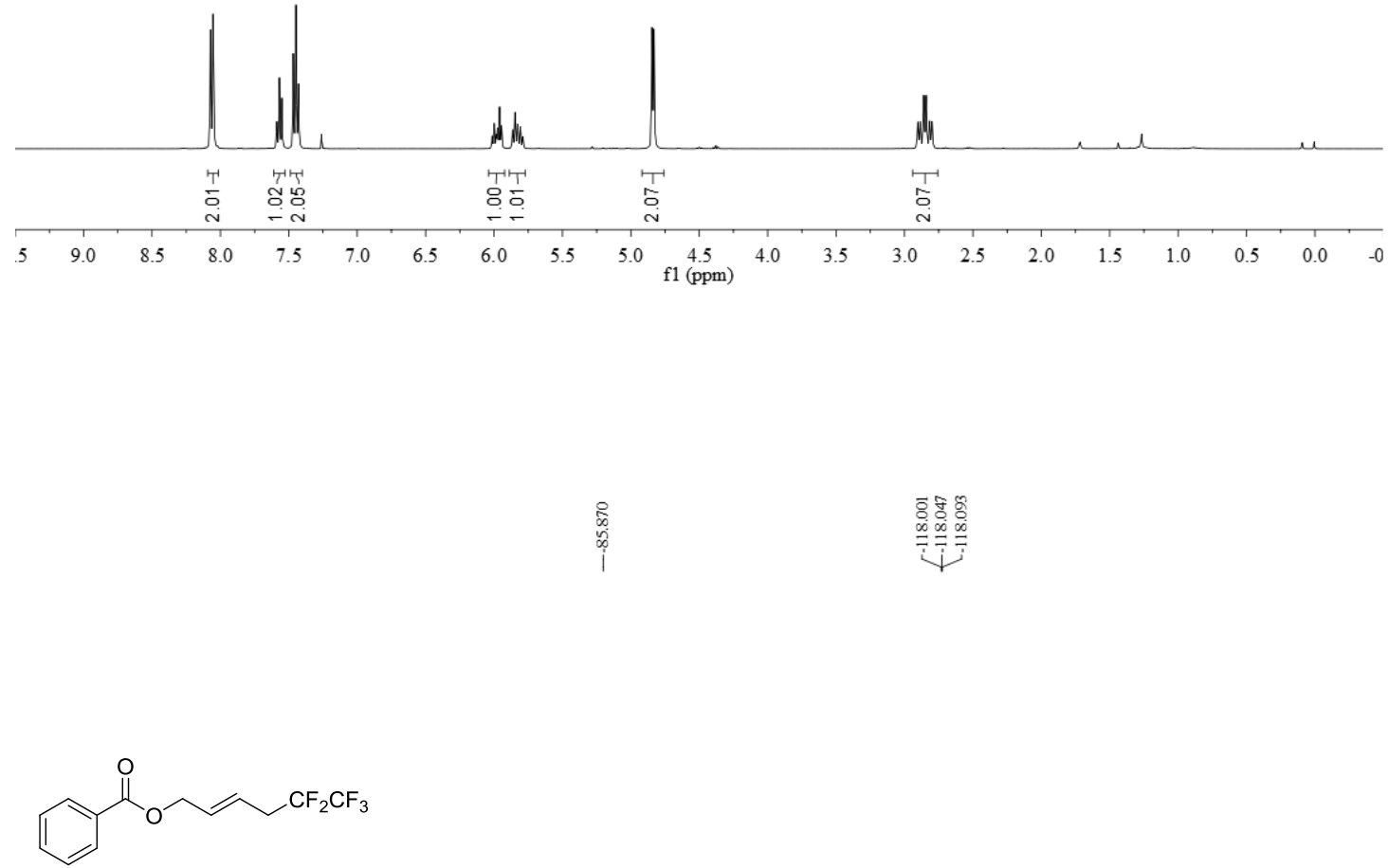

2w $\left({ }^{19} \mathrm{~F}\right.$ NMR, $\left.\mathrm{CDCl}_{3}, 376 \mathrm{MHz}\right)$

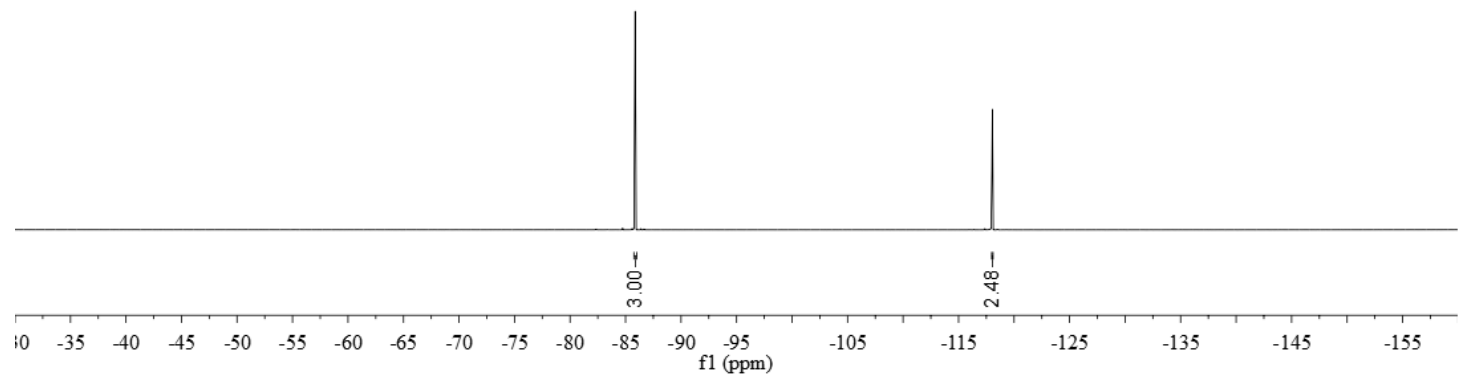




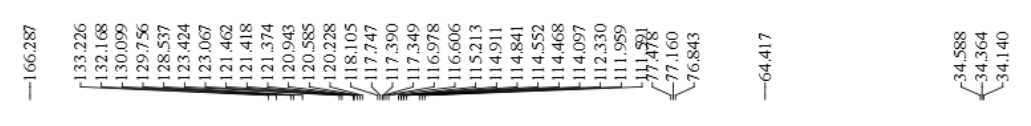

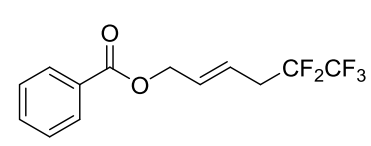

2w $\left({ }^{13} \mathrm{C}\right.$ NMR, $\left.\mathrm{CDCl}_{3}, 101 \mathrm{MHz}\right)$
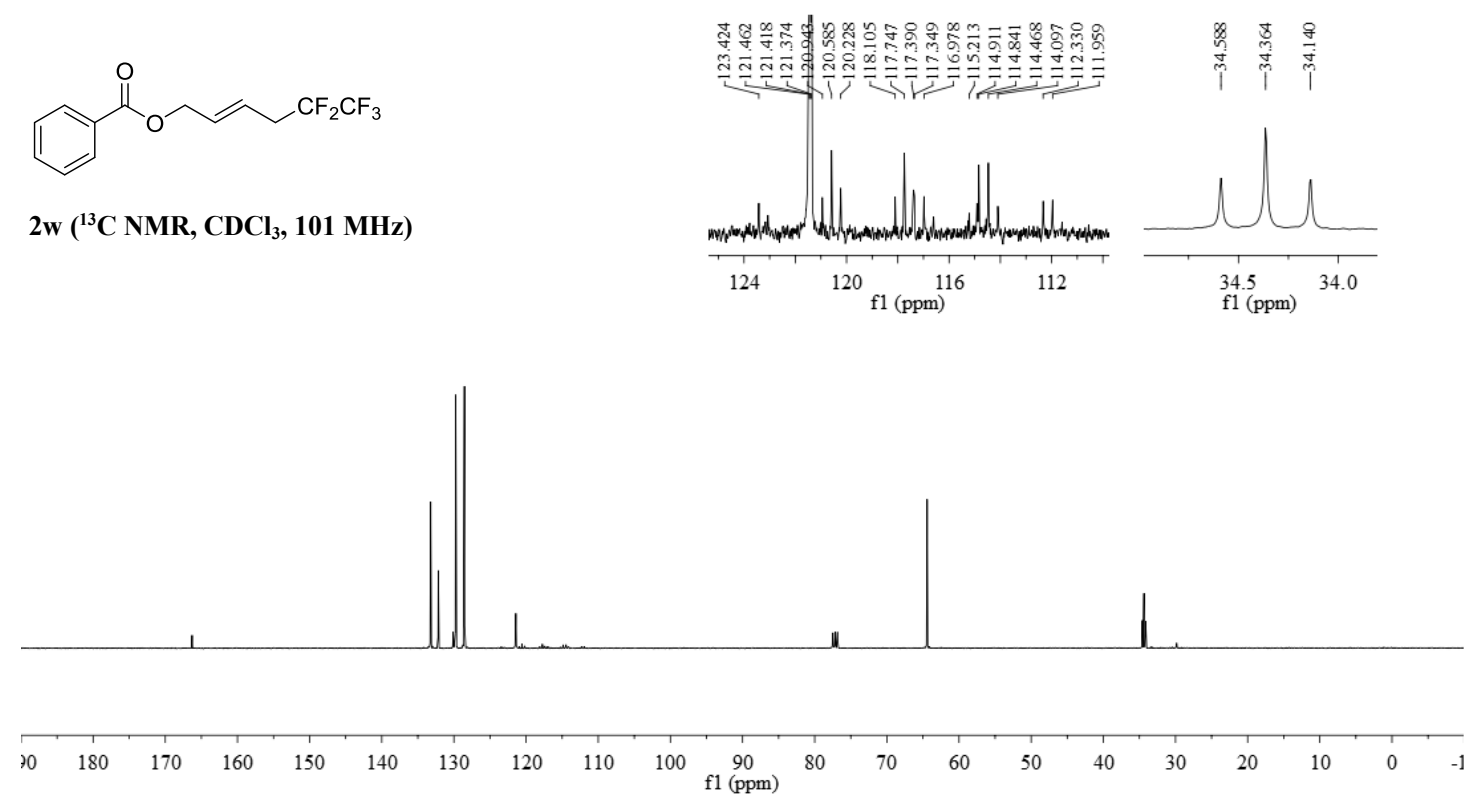

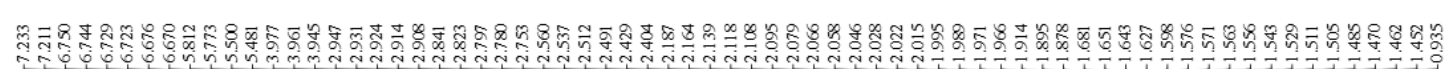

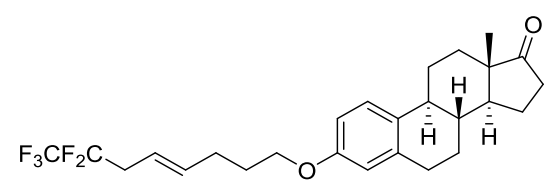

2x ('H NMR, $\left.\mathrm{CDCl}_{3}, 400 \mathrm{MHz}\right)$

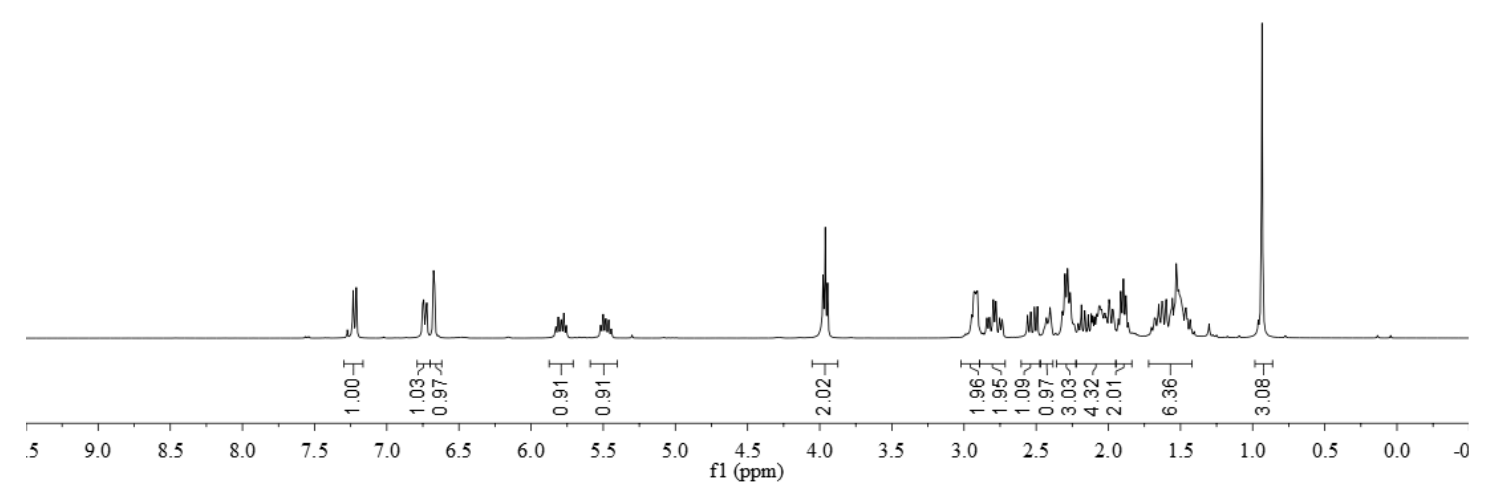

S58 

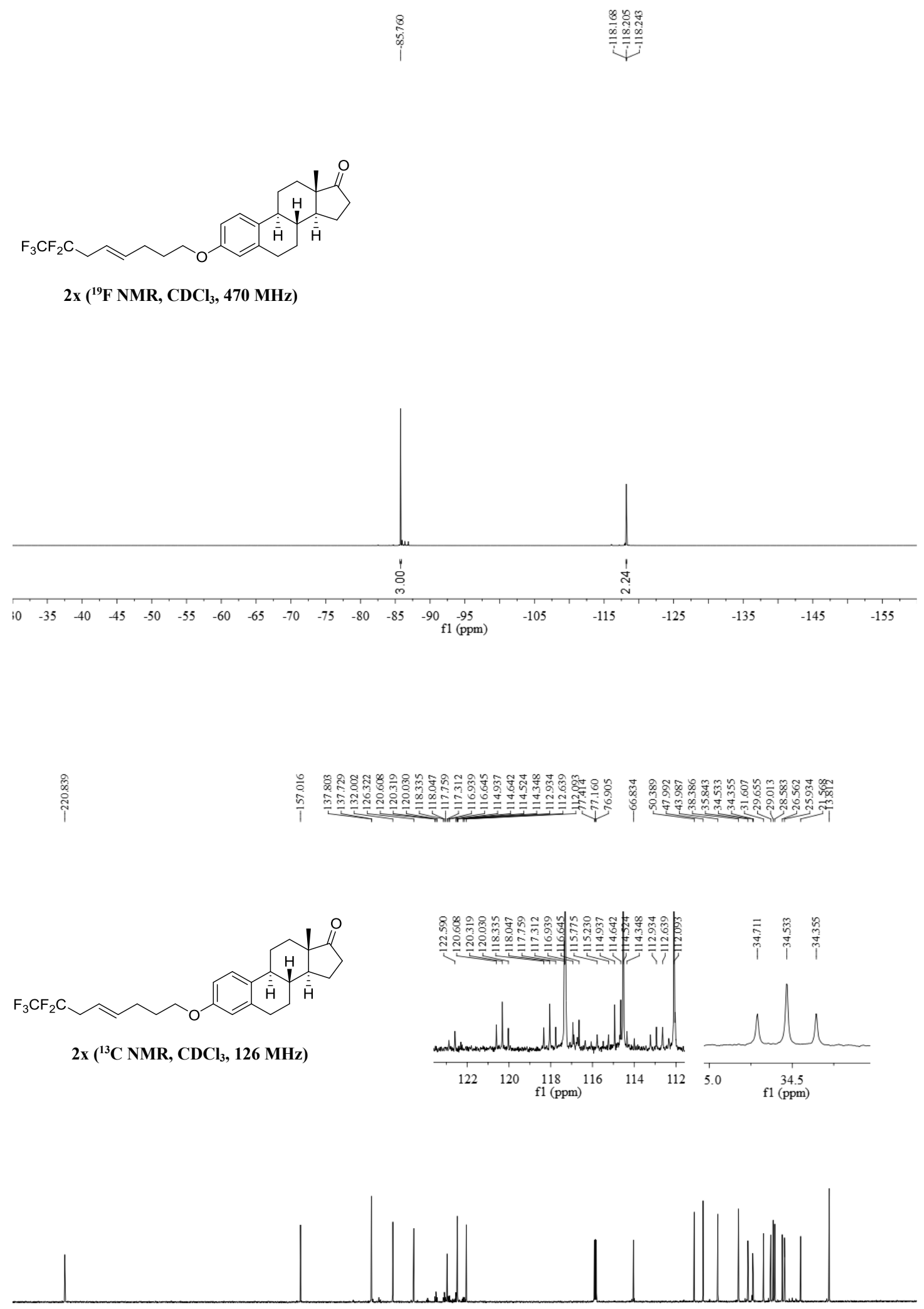

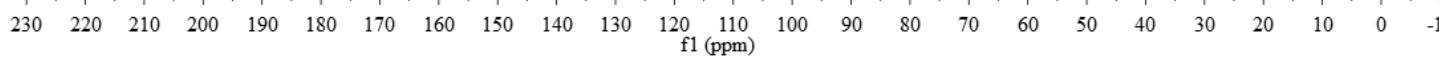




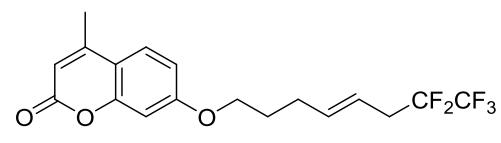

$2 y\left({ }^{1} \mathrm{H}\right.$ NMR, $\left.\mathrm{CDCl}_{3}, 400 \mathrm{MHz}\right)$
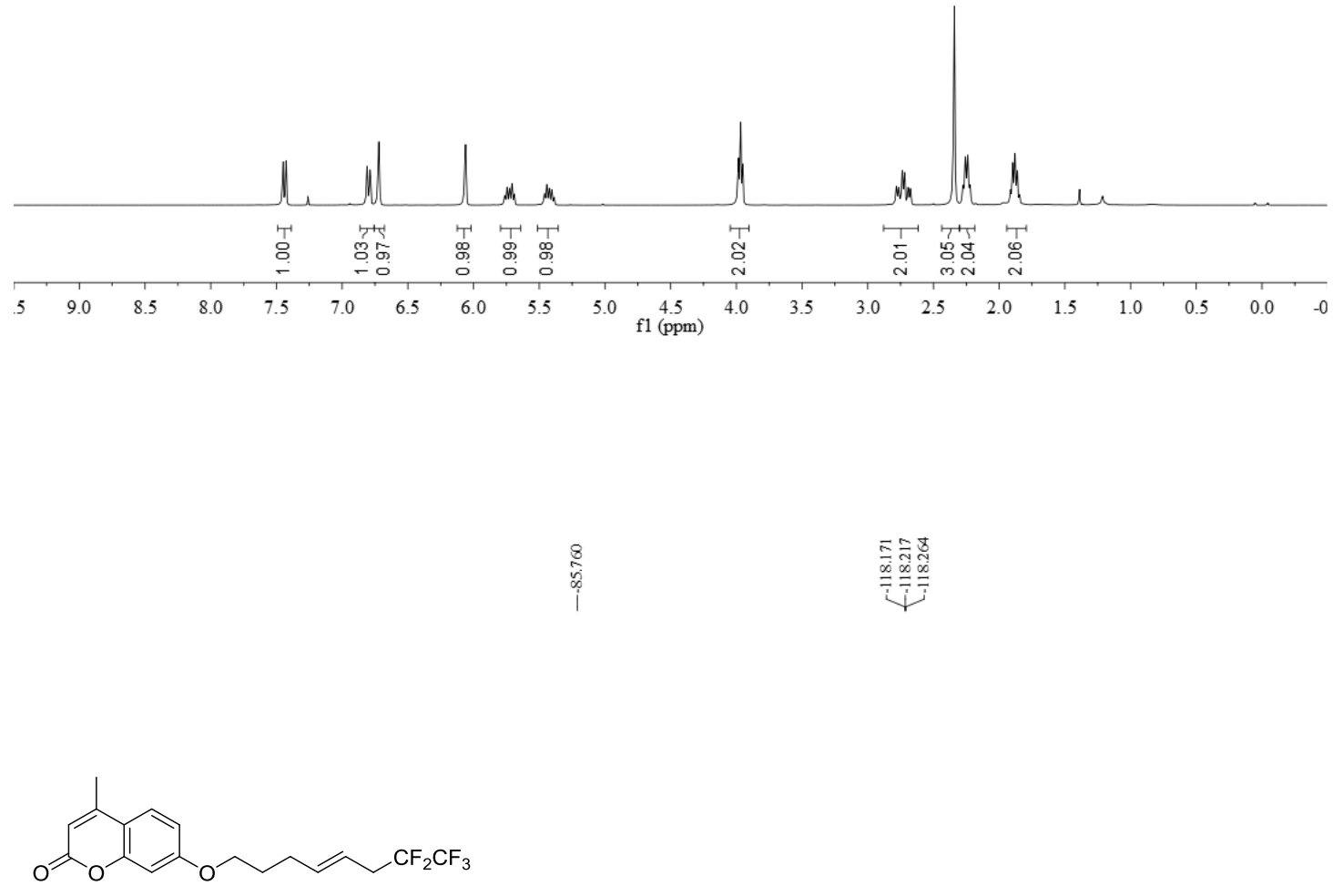

$2 \mathrm{y}\left({ }^{19} \mathrm{~F} \mathrm{NMR}, \mathrm{CDCl}_{3}, 376 \mathrm{MHz}\right)$

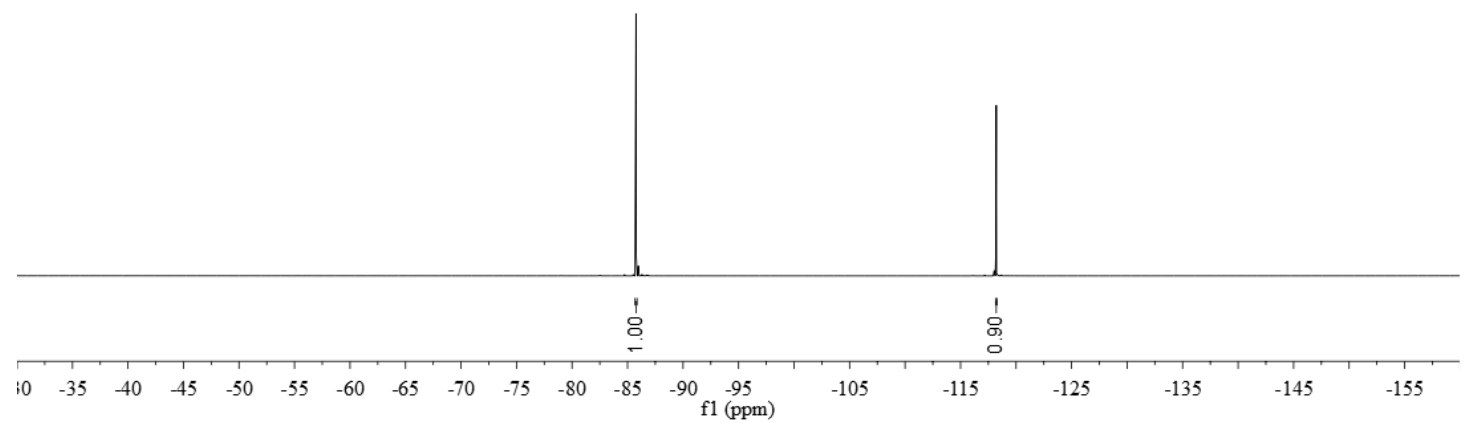




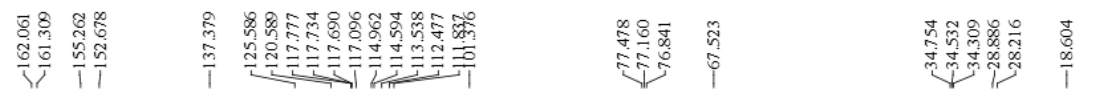
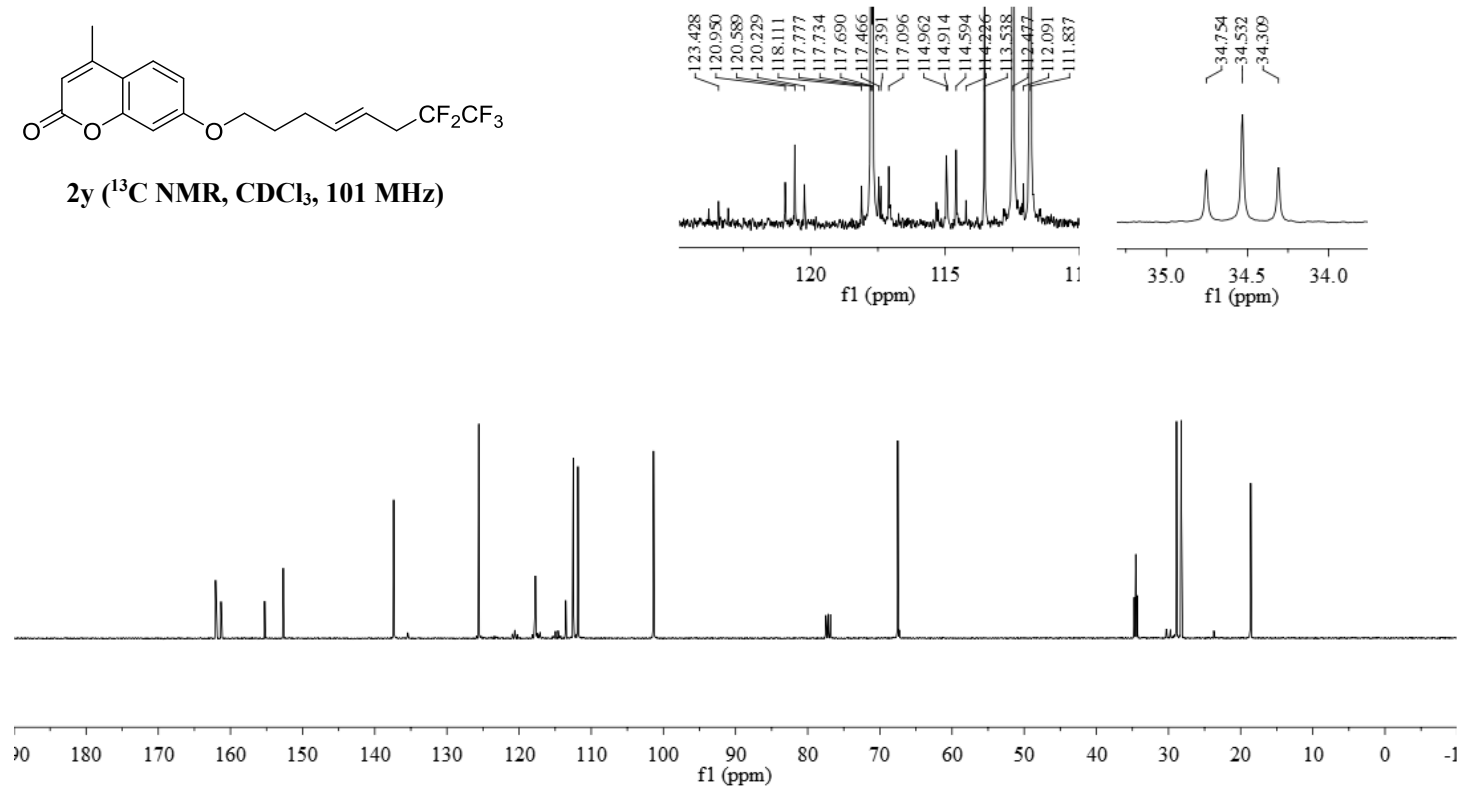

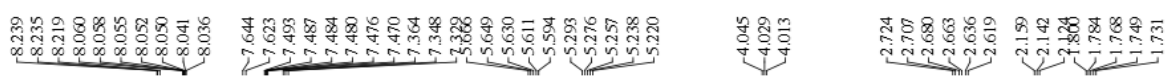<smiles>O=c1c(OCCC=CCC(F)(F)F)c(-c2ccccc2)oc2ccccc12</smiles>

2z ('H NMR, $\left.\mathrm{CDCl}_{3}, 400 \mathrm{MHz}\right)$

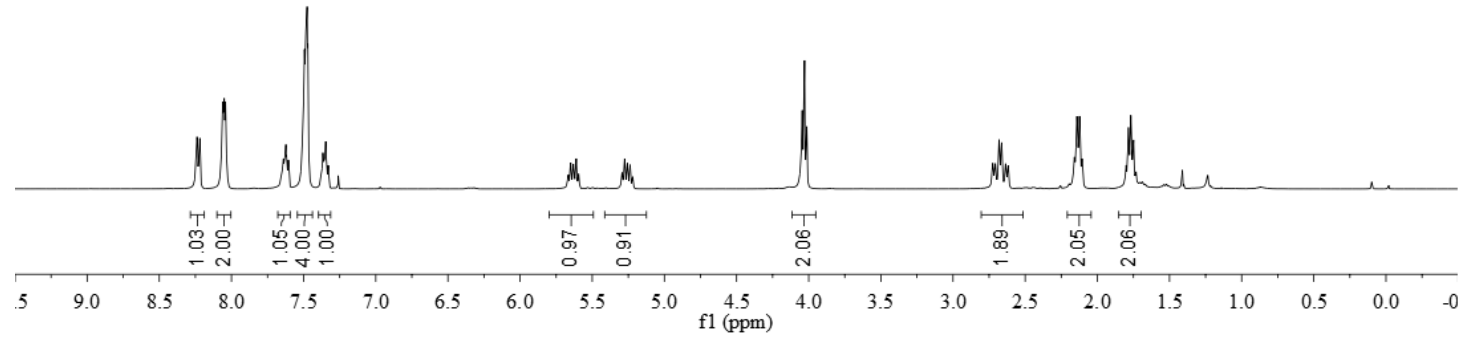



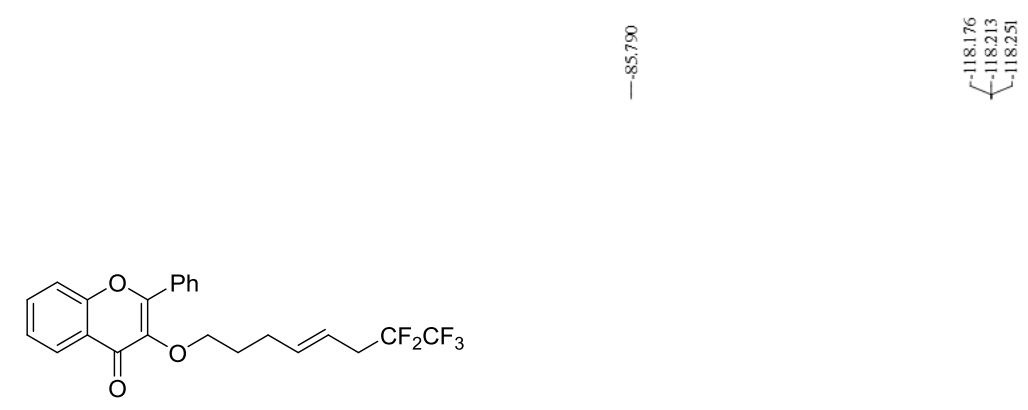

$2 \mathrm{z}\left({ }^{19} \mathrm{~F} \mathrm{NMR}, \mathrm{CDCl}_{3}, 470 \mathrm{MHz}\right)$

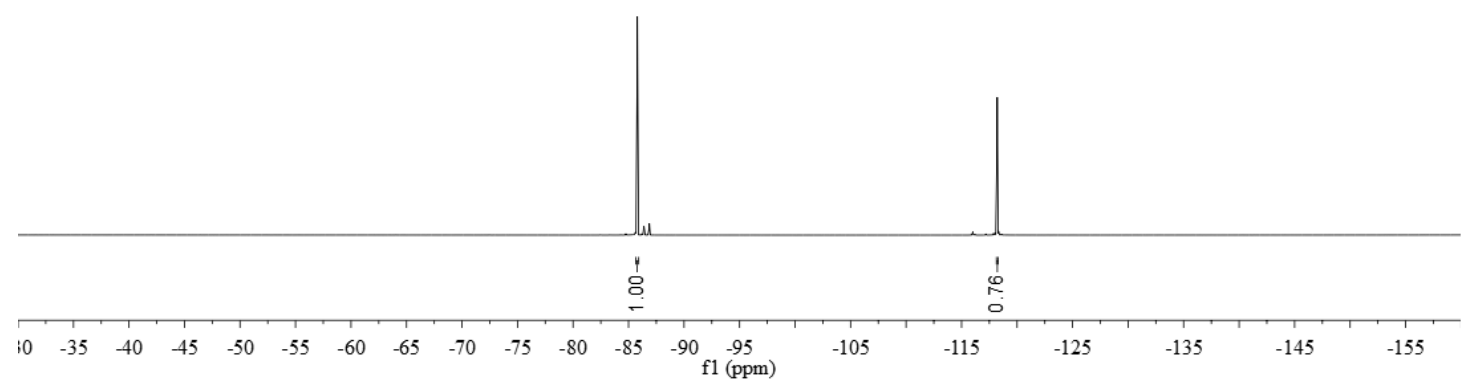

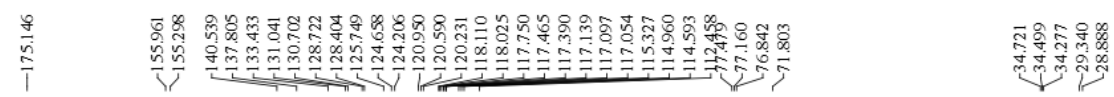

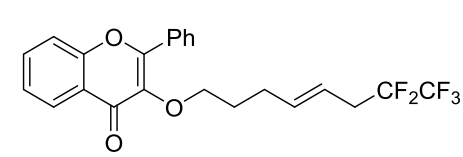

$2 \mathrm{z}\left({ }^{13} \mathrm{C} \mathrm{NMR}, \mathrm{CDCl}_{3}, 101 \mathrm{MHz}\right)$
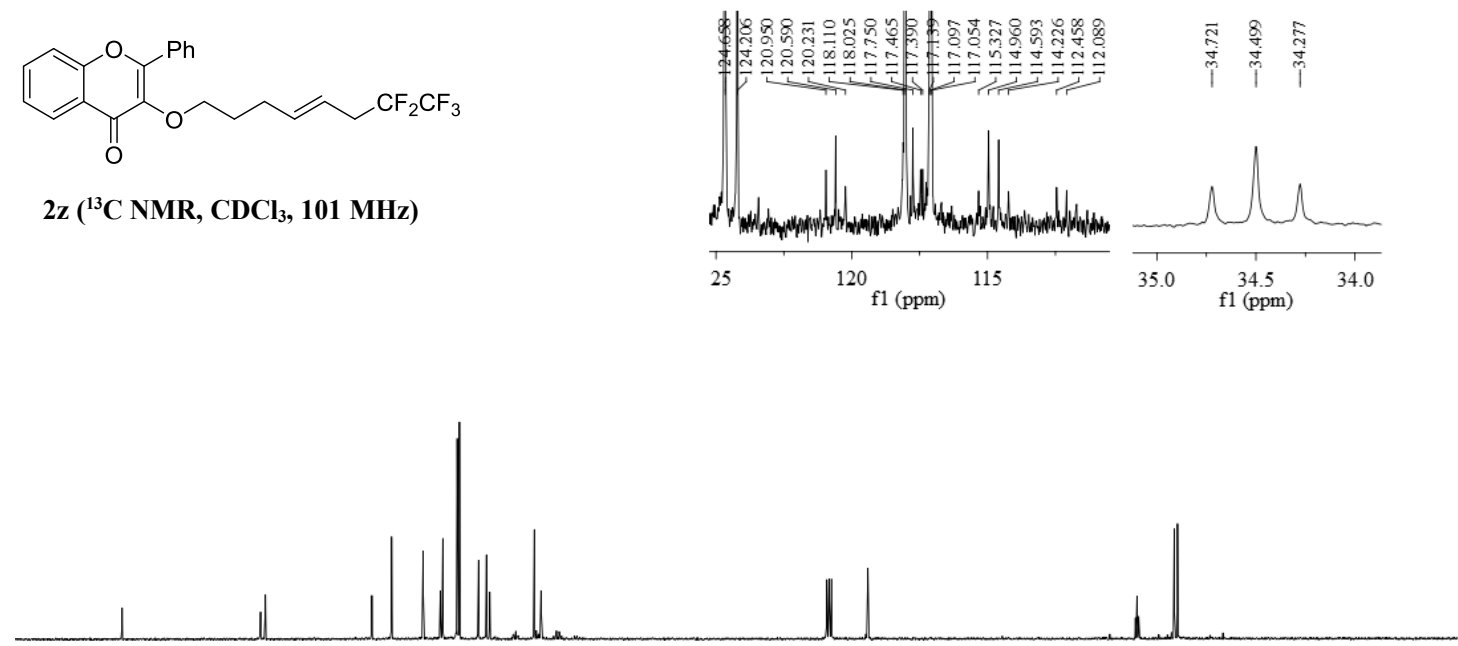

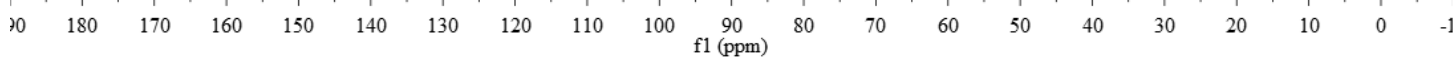




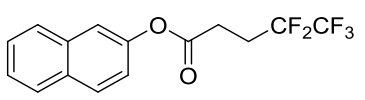

2aa ('H NMR, $\mathrm{CDCl}_{3}, 100 \mathrm{MHz}$ )
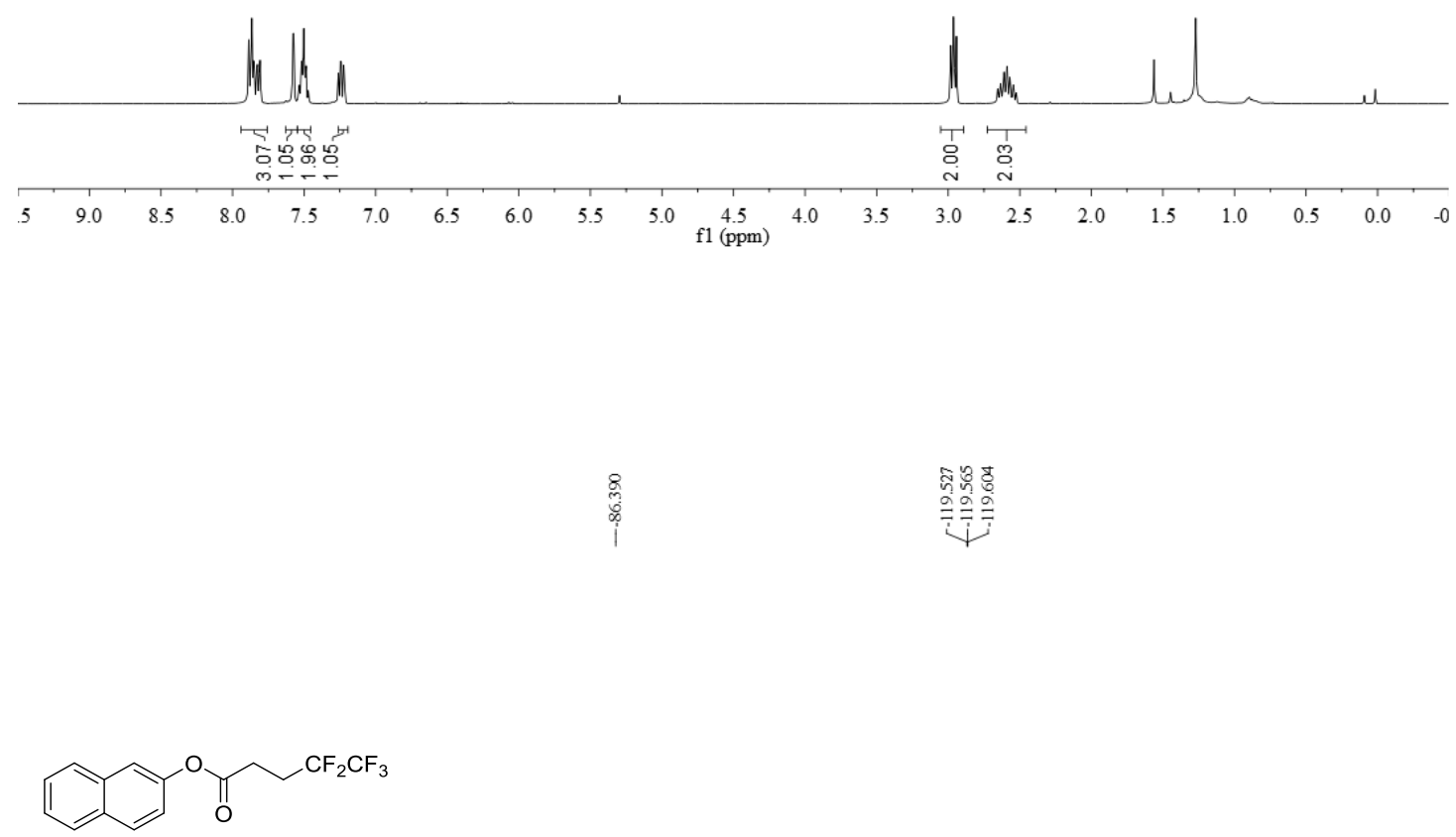

2aa $\left({ }^{19} \mathrm{~F}\right.$ NMR, $\left.\mathrm{CDCl}_{3}, 470 \mathrm{MHz}\right)$

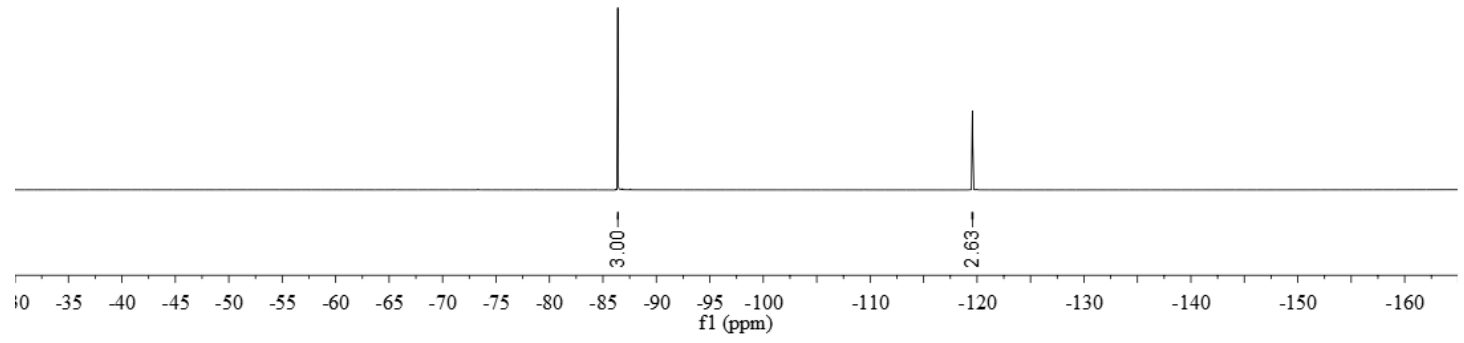




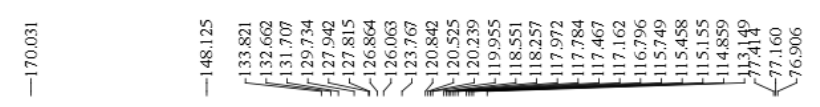

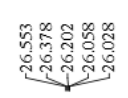

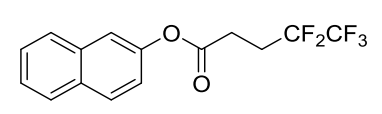

2aa $\left({ }^{13} \mathrm{C} \mathrm{NMR}, \mathrm{CDCl}_{3}, 126 \mathrm{MHz}\right)$
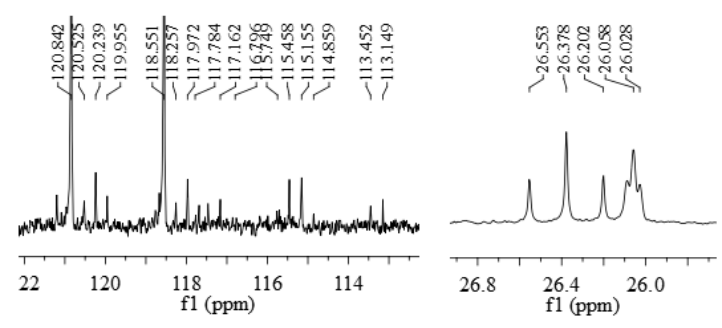
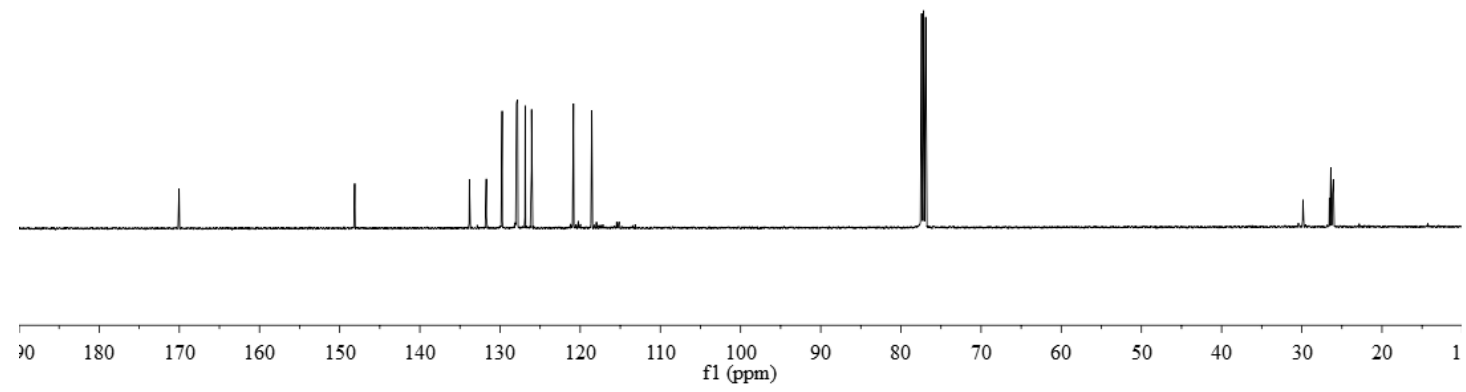

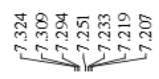

帝

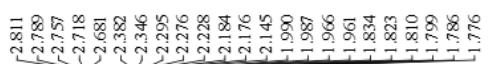

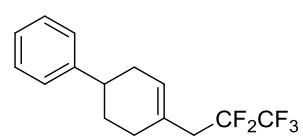

2ab ( ${ }^{1} \mathrm{H}$ NMR, $\left.\mathrm{CDCl}_{3}, 500 \mathrm{MHz}\right)$

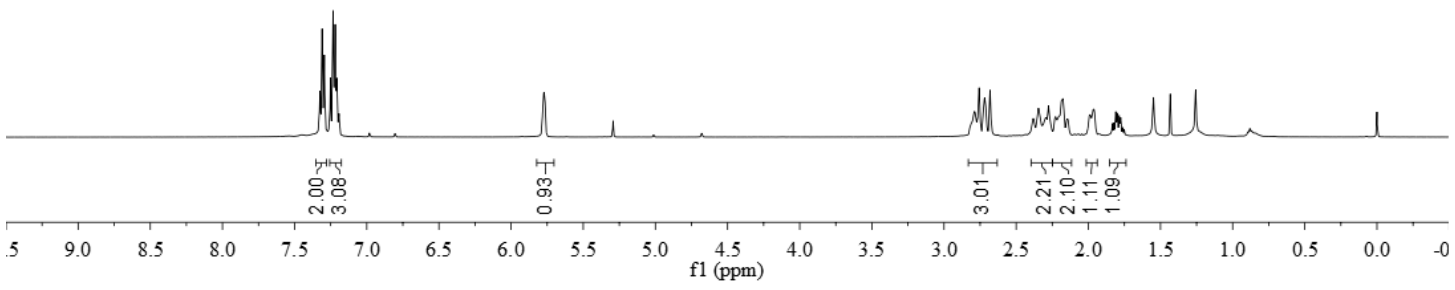

S64 

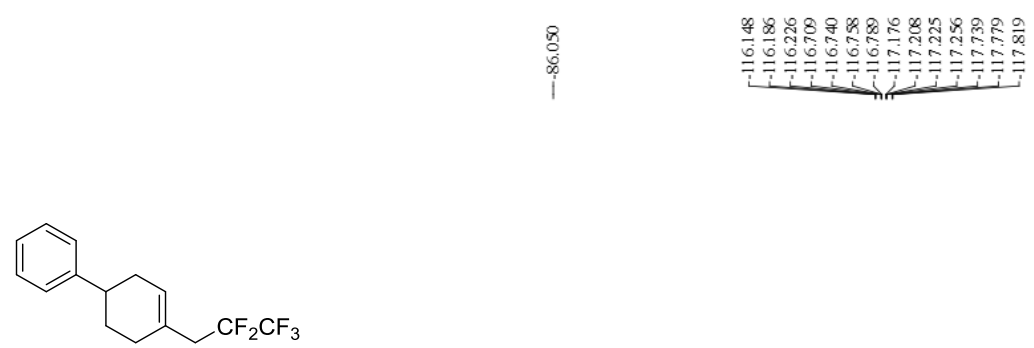

2ab $\left({ }^{19} \mathrm{~F}\right.$ NMR, $\left.\mathrm{CDCl}_{3}, 470 \mathrm{MHz}\right)$

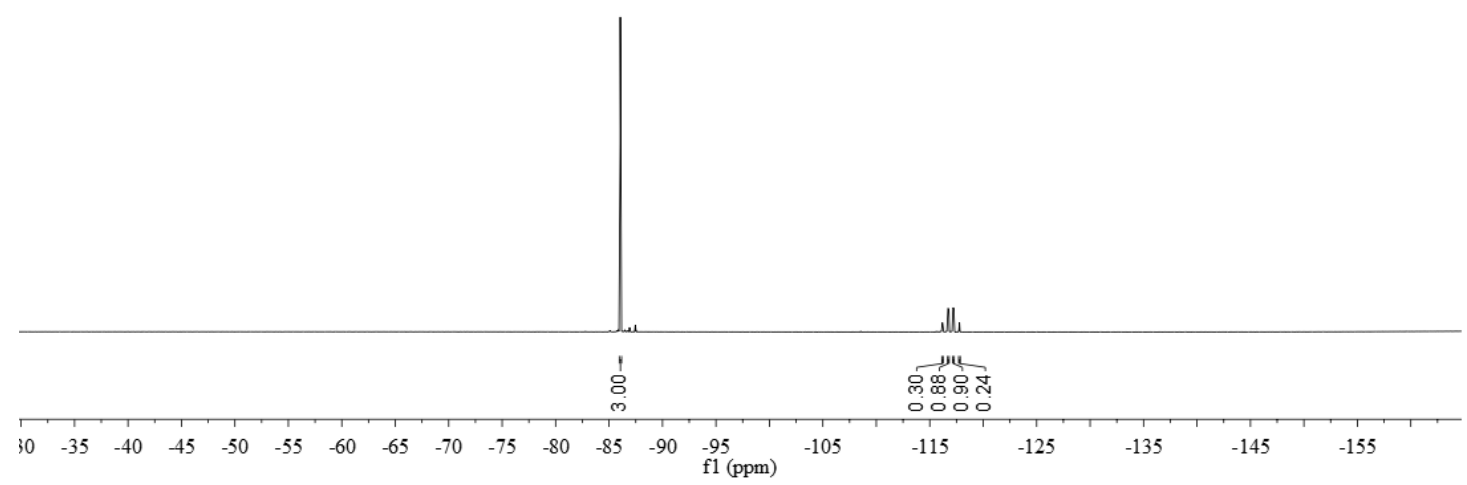

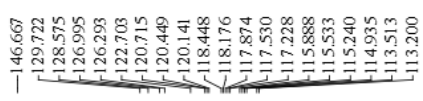

声省递

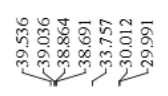

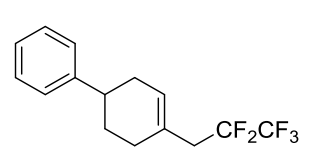

2ab $\left({ }^{13} \mathrm{C} \mathrm{NMR}, \mathrm{CDCl}_{3}, 126 \mathrm{MHz}\right)$
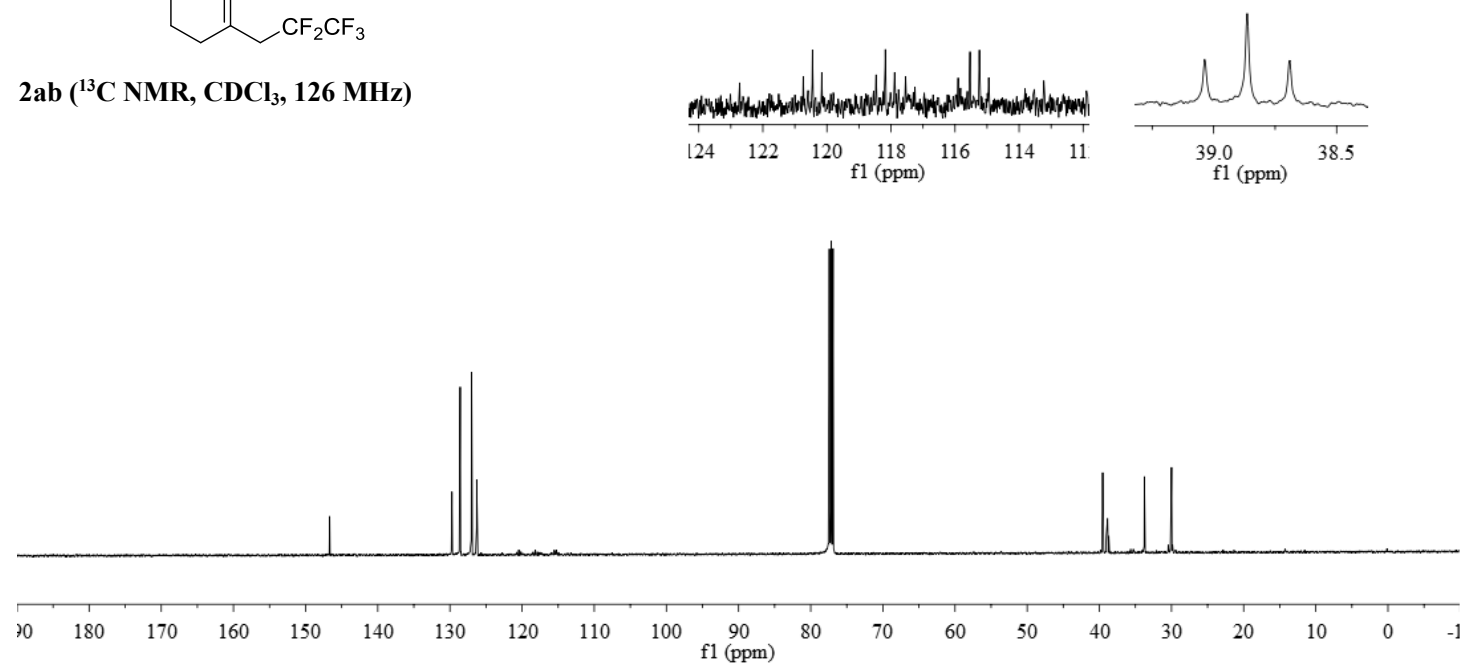

S65 


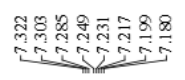

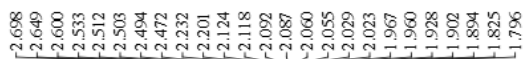

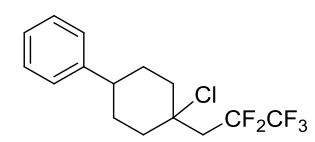

2ab" ( ${ }^{1} \mathrm{H}$ NMR, $\left.\mathrm{CDCl}_{3}, 400 \mathrm{MHz}\right)$
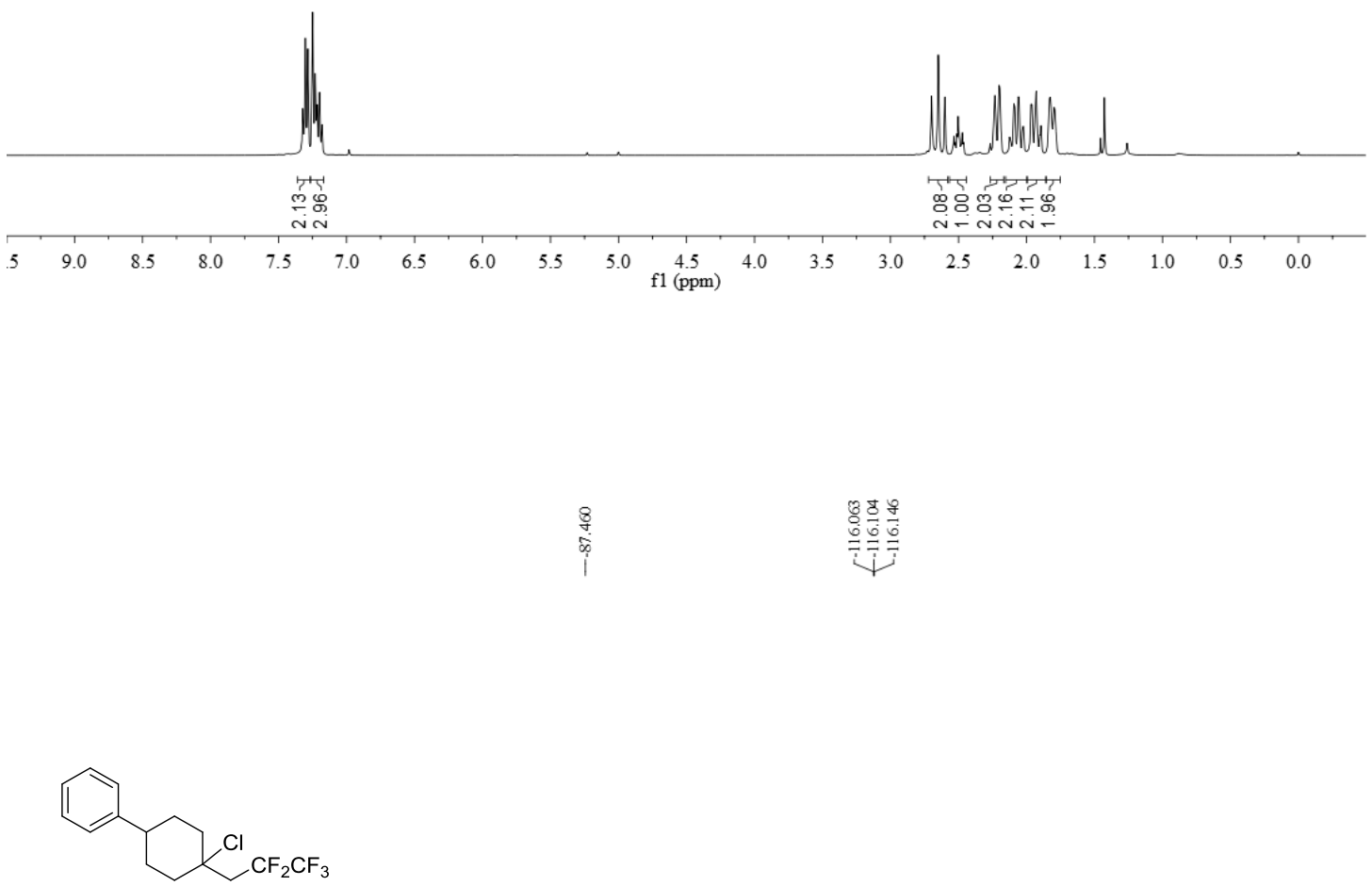

2ab" $\left({ }^{19} \mathrm{~F} \mathrm{NMR,} \mathrm{CDCl}_{3}, 470 \mathrm{MHz}\right)$

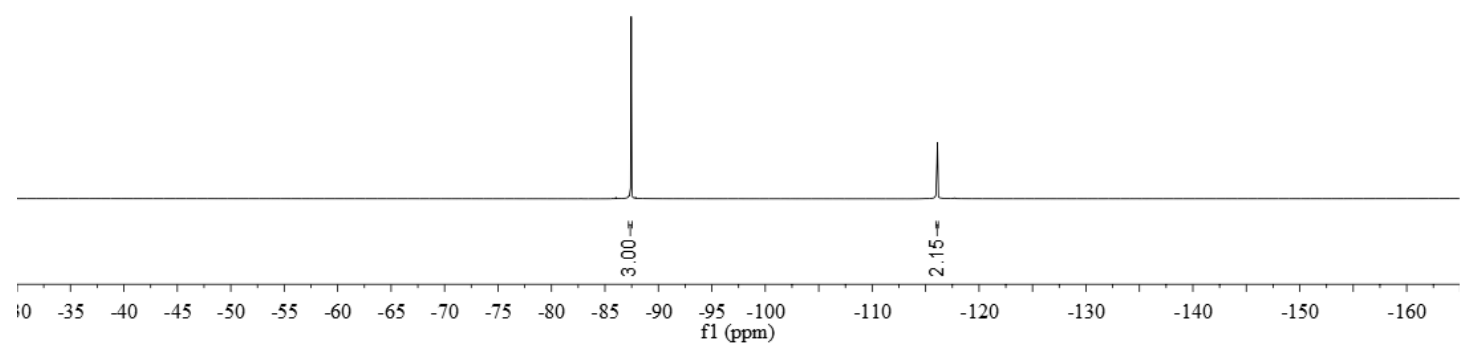

S66 


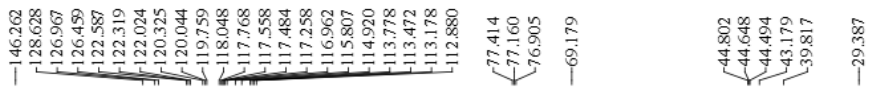

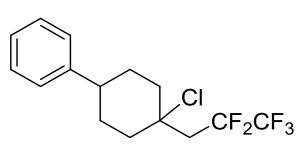

2ab" $\left({ }^{13} \mathrm{C} \mathrm{NMR,} \mathrm{CDCl}_{3}, 126 \mathrm{MHz}\right)$

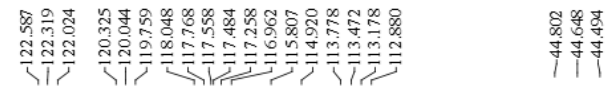

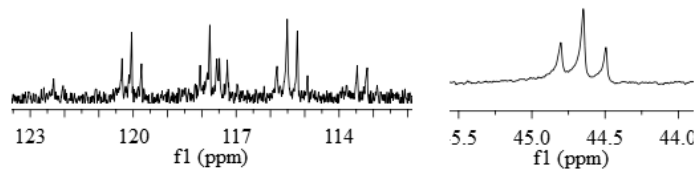

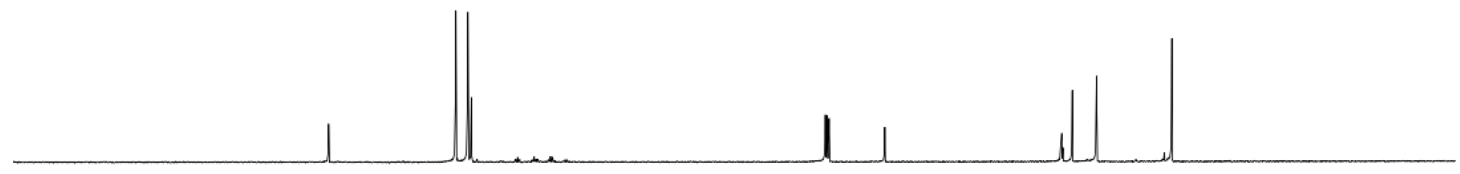

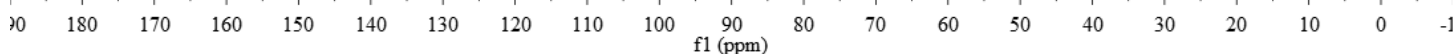
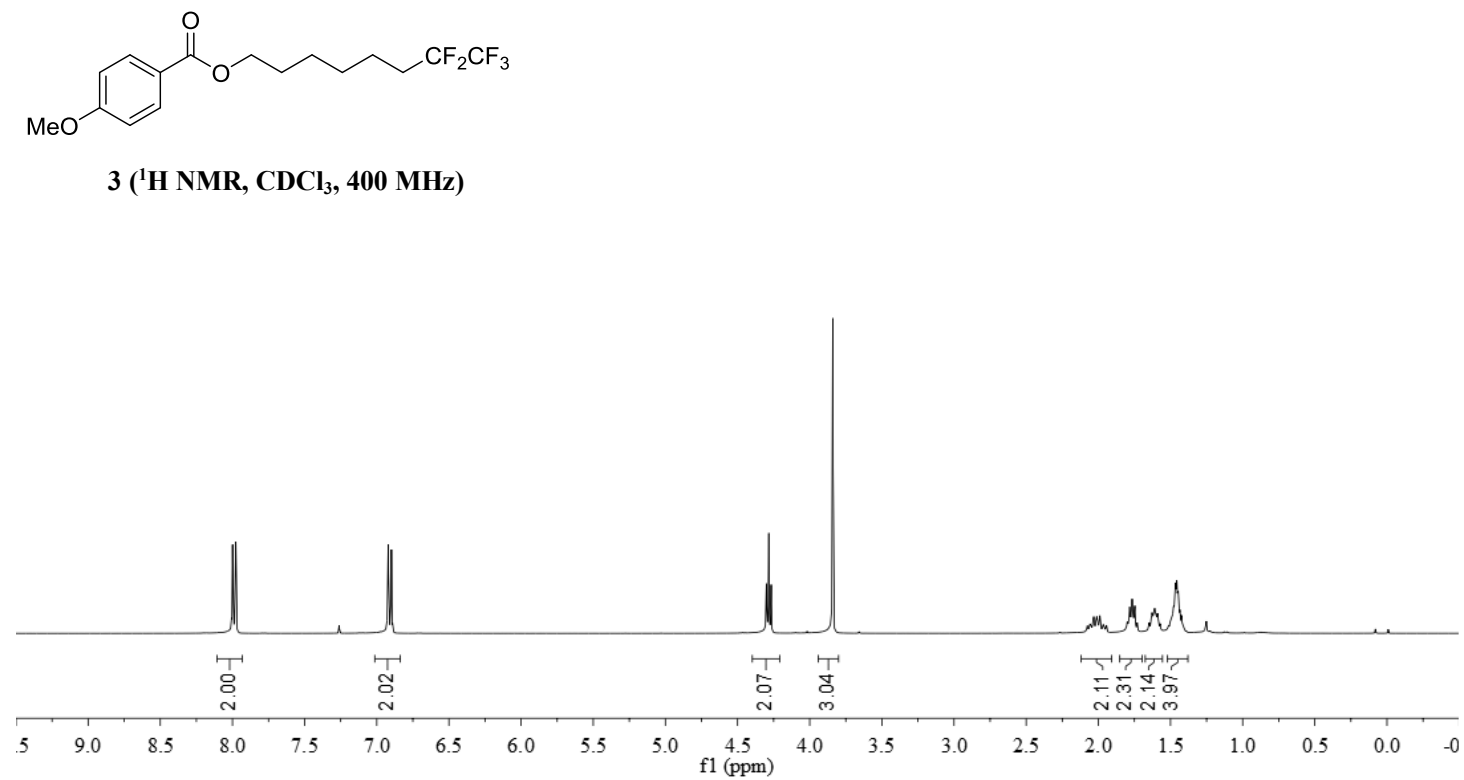

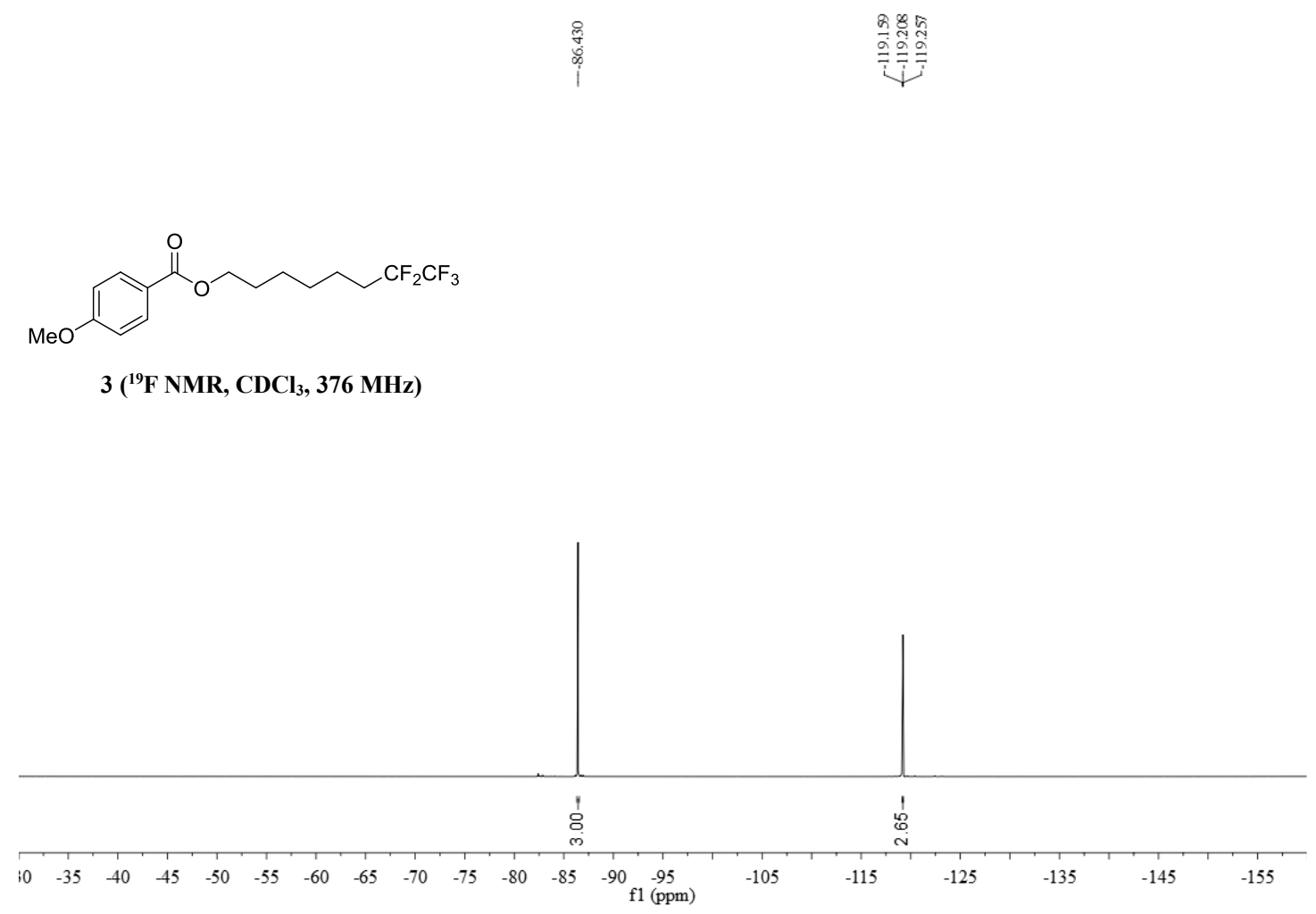
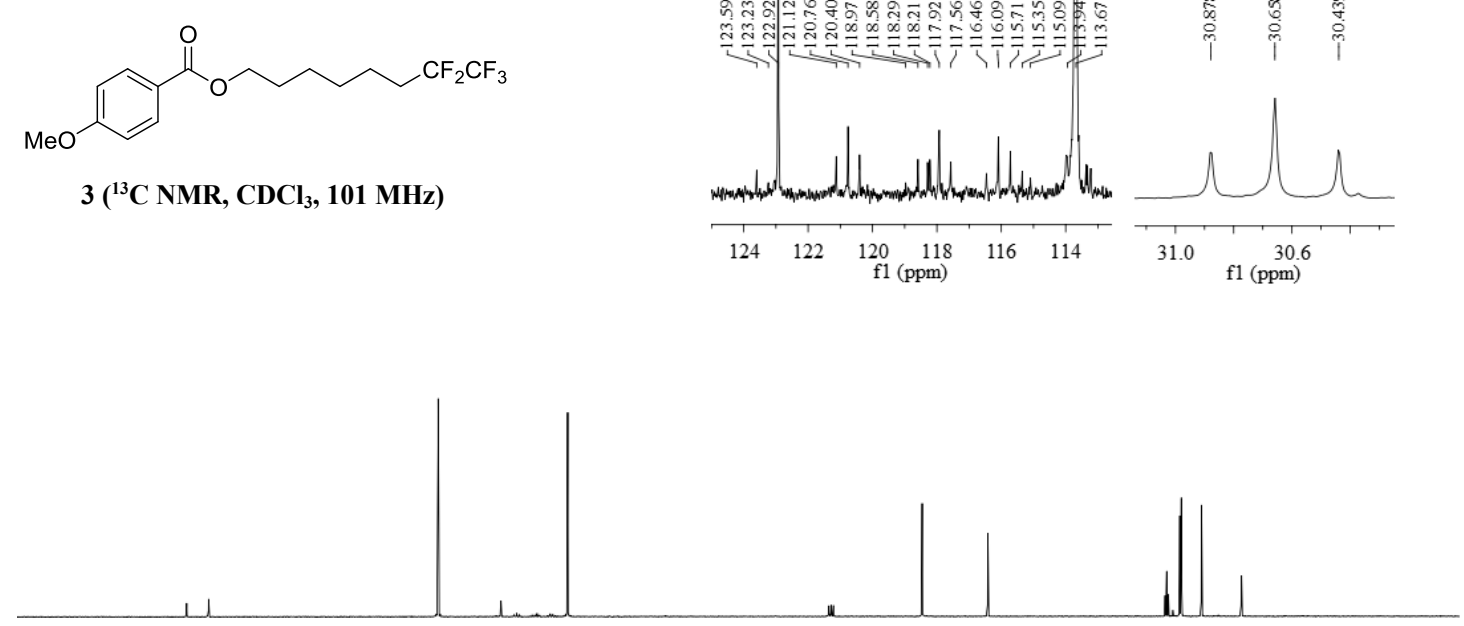

$30 \quad 180 \quad 170 \quad 160$
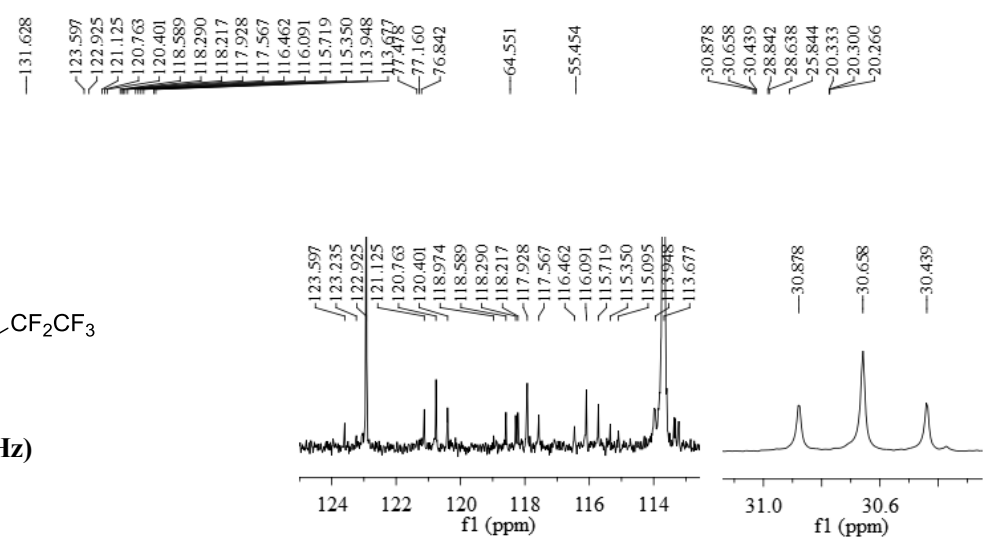

$00 \quad 90$ 


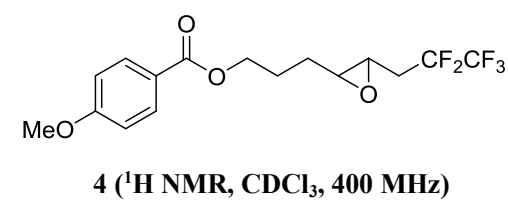

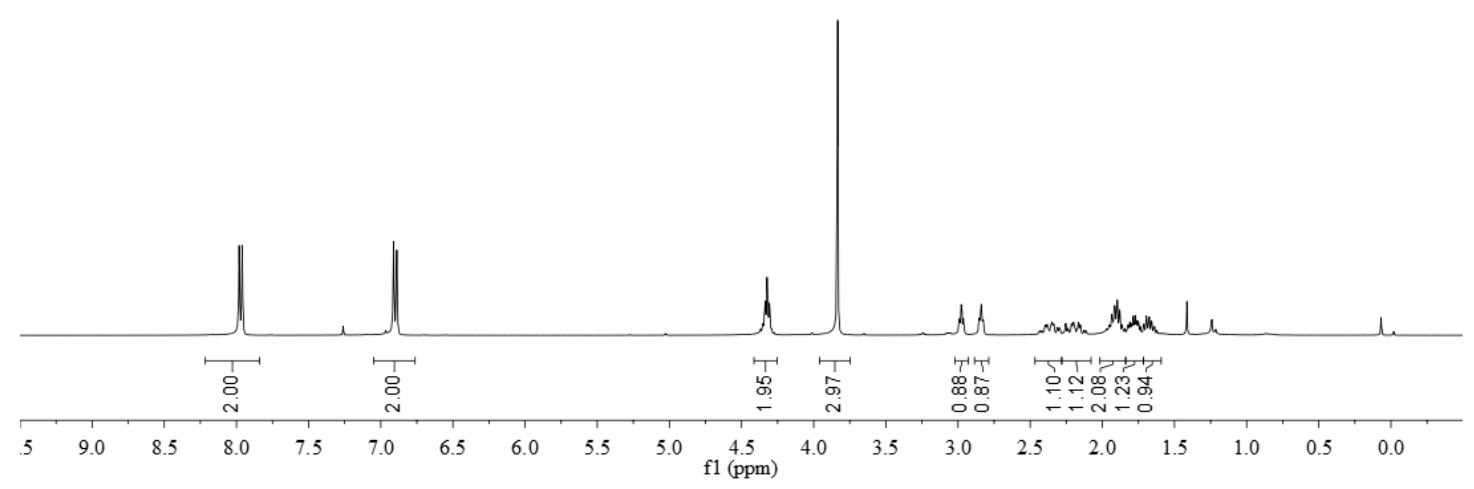

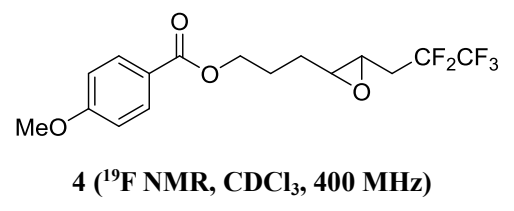
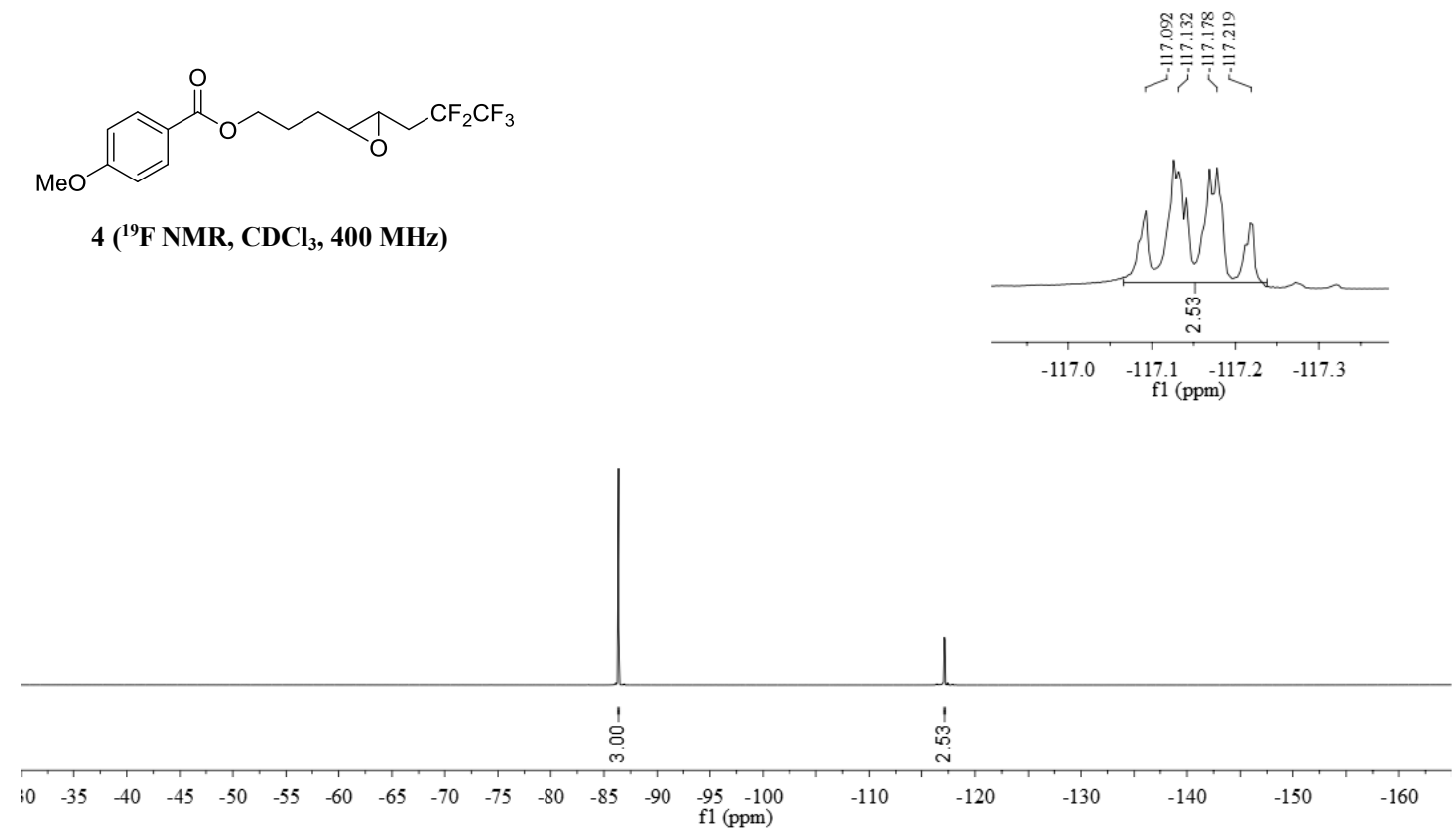

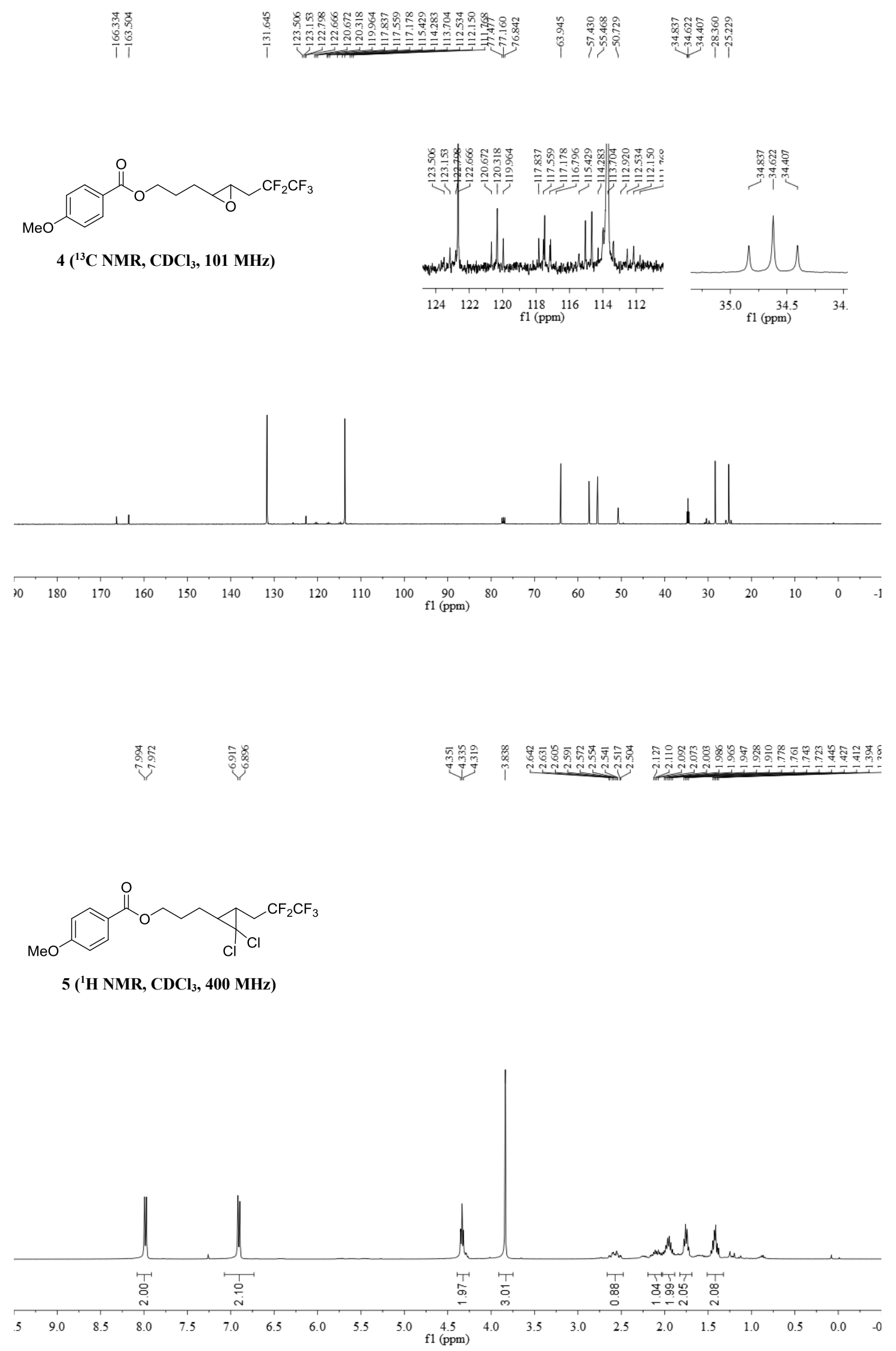


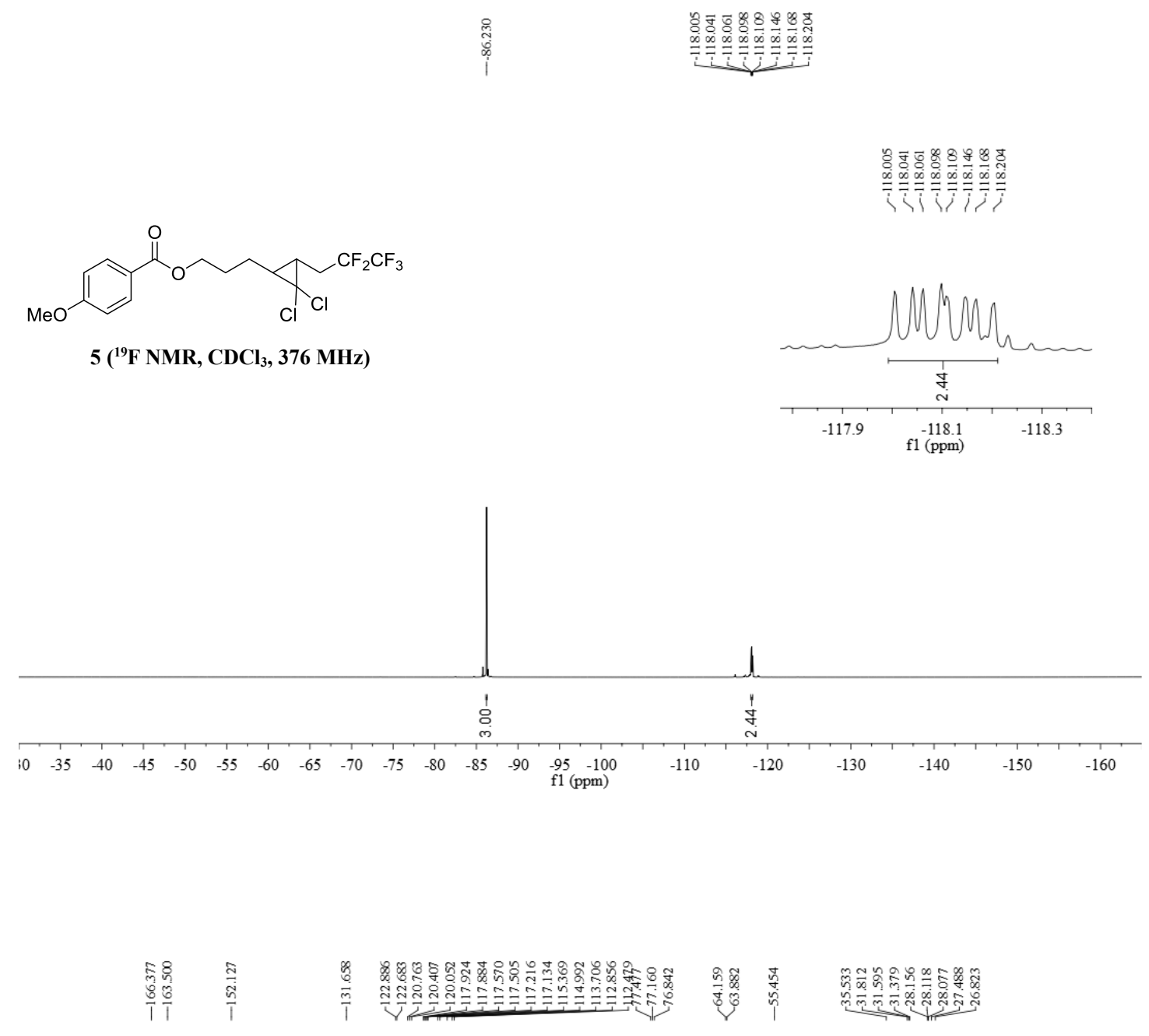

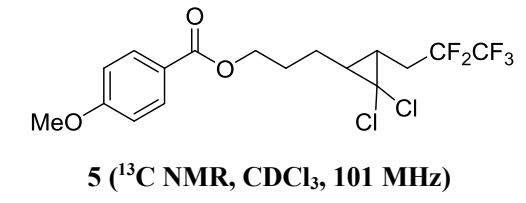

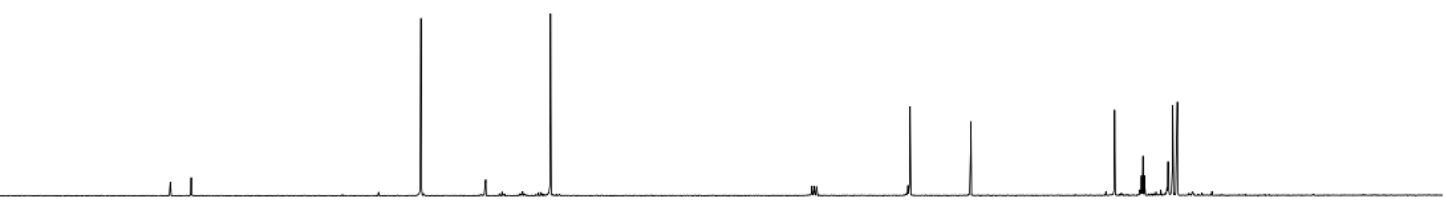

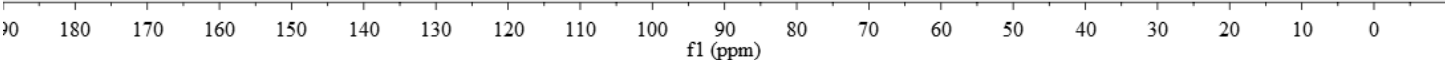



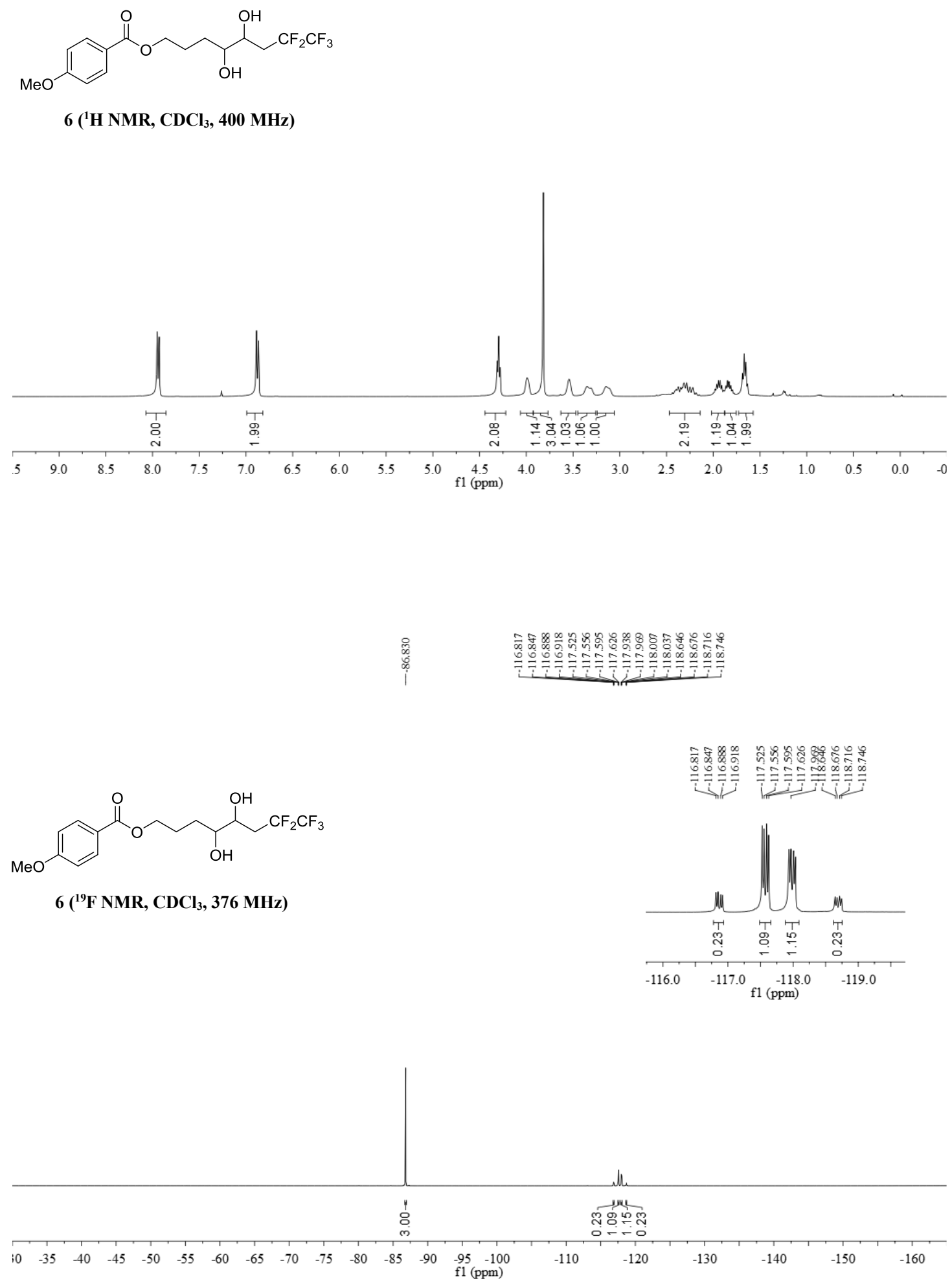

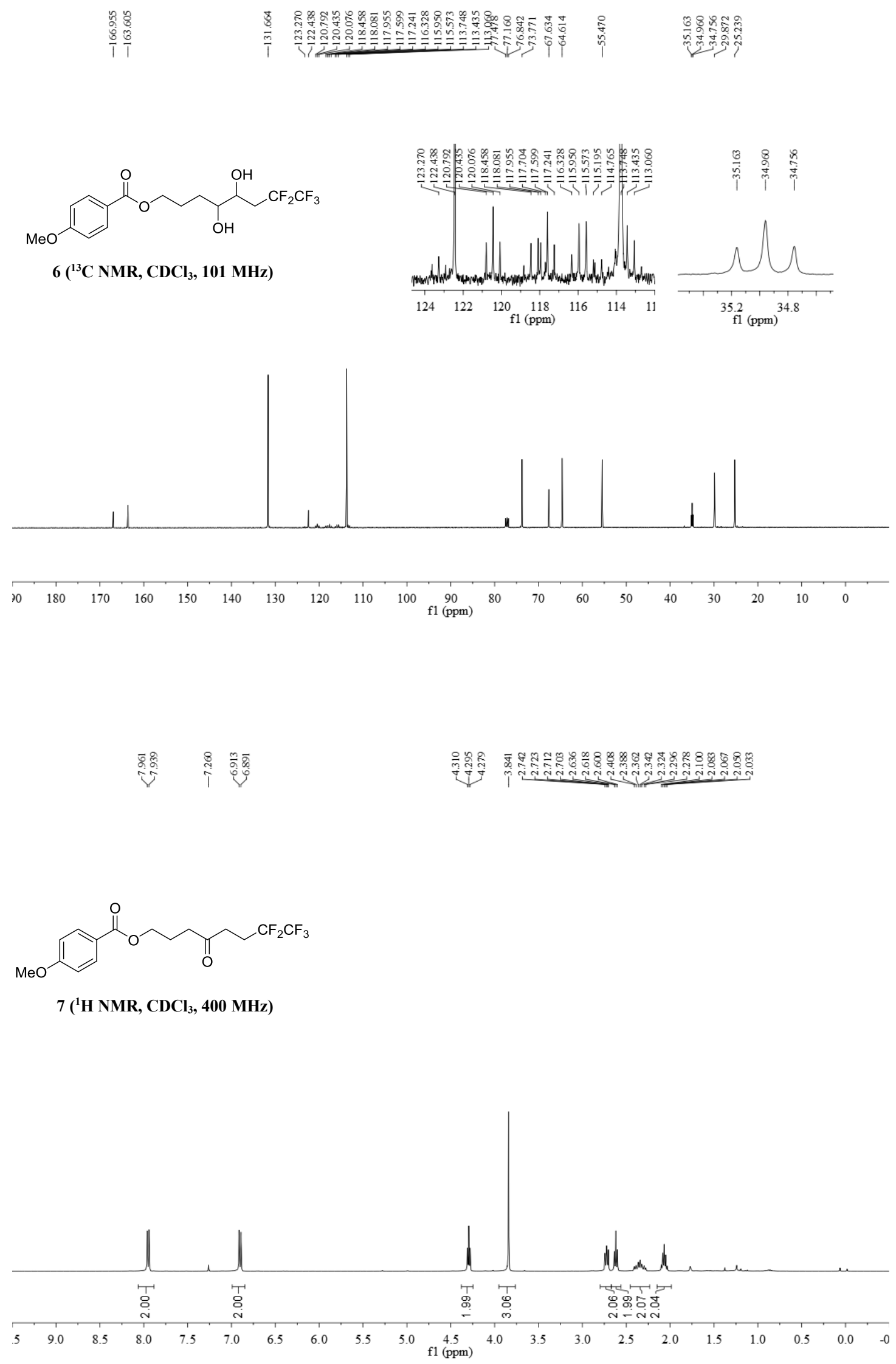

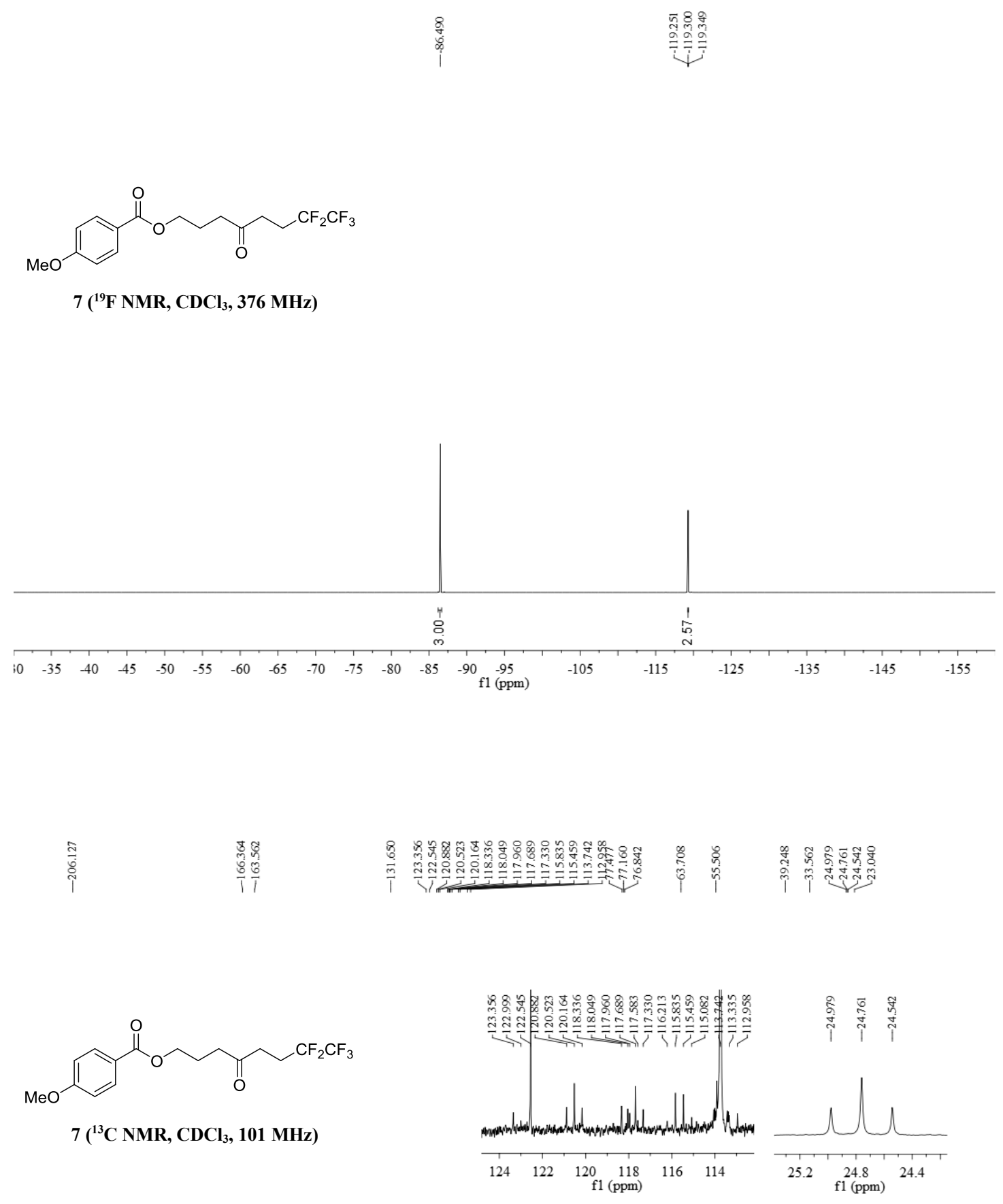

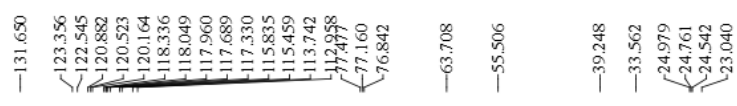
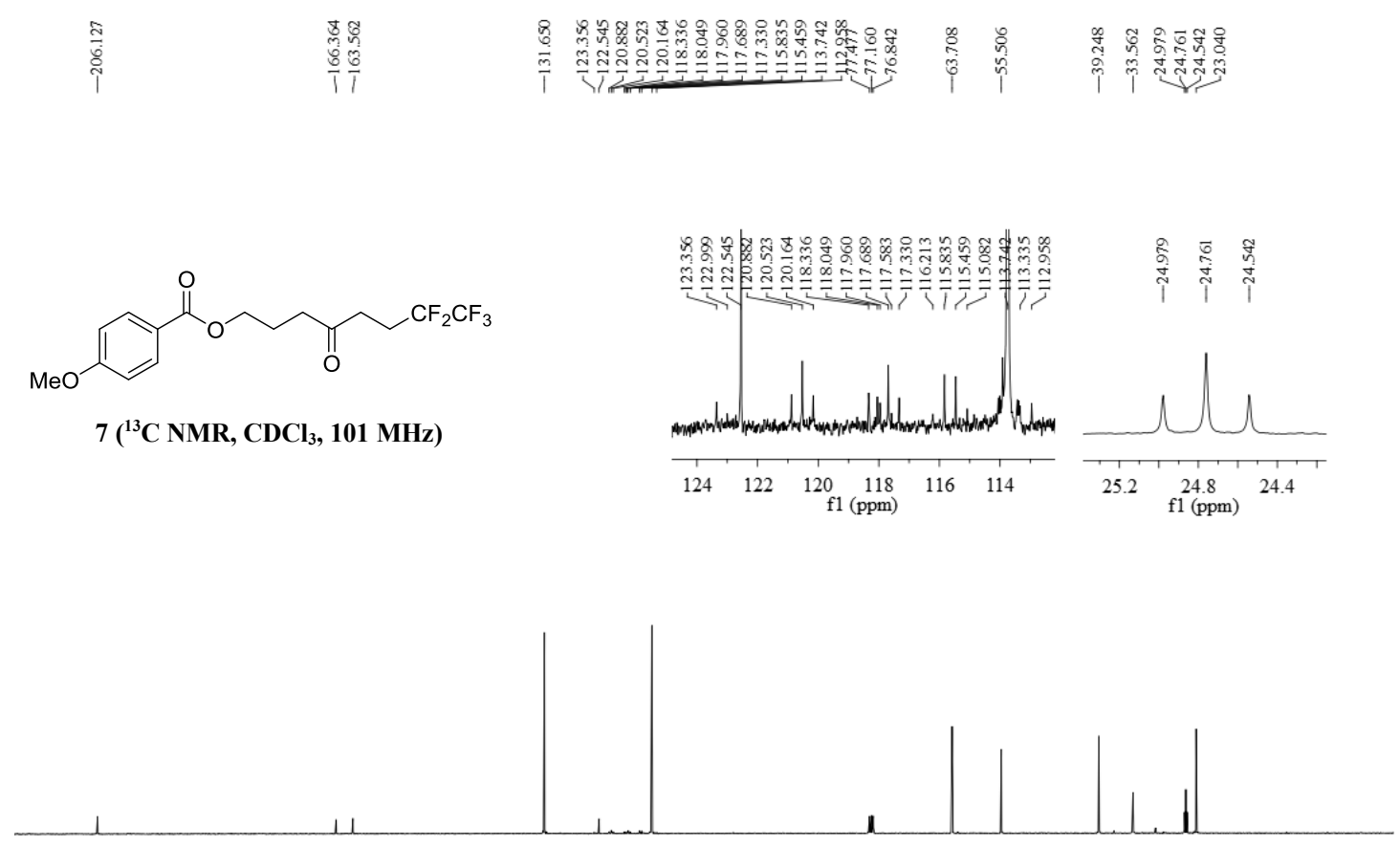

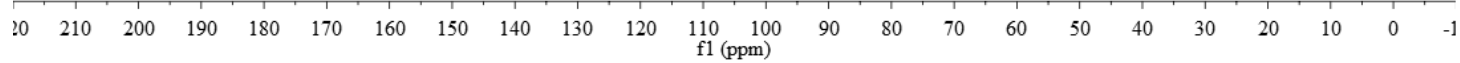




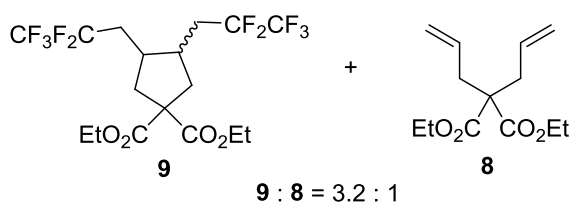

('H NMR, $\left.\mathrm{CDCl}_{3}, 400 \mathrm{MHz}\right)$

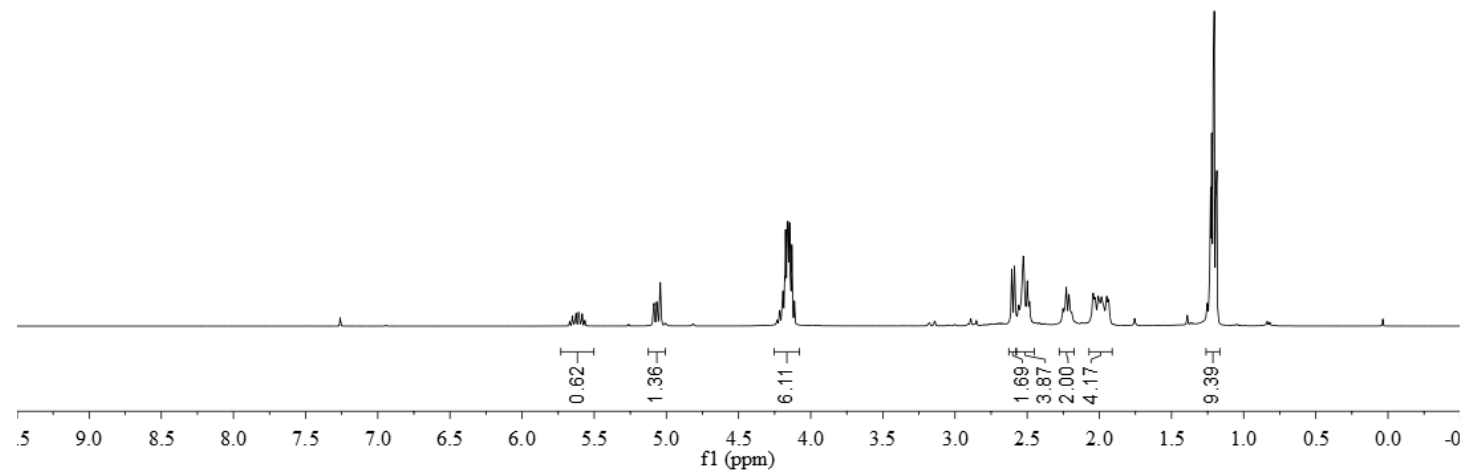

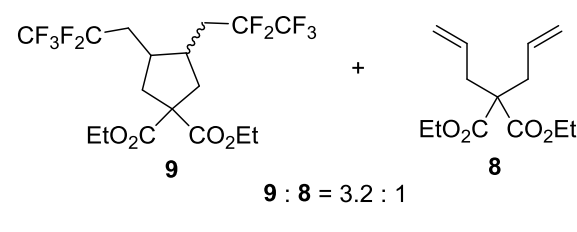

$\left({ }^{19} \mathrm{~F} \mathrm{NMR}, \mathrm{CDCl}_{3}, 376 \mathrm{MHz}\right)$
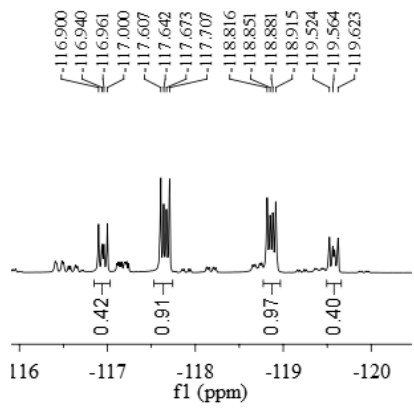

elde

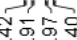

$\begin{array}{lllllllllllllllllllllllll}0 & -35 & -40 & -45 & -50 & -55 & -60 & -65 & -70 & -75 & -80 & -85 & -90 & -95 & -105 & -115 & -125 & -135 & -145 & -155\end{array}$ 


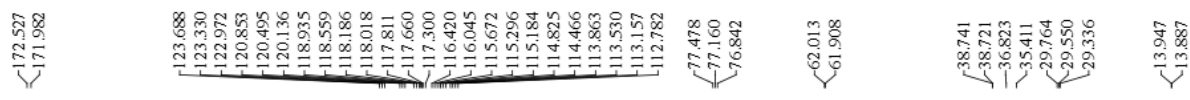

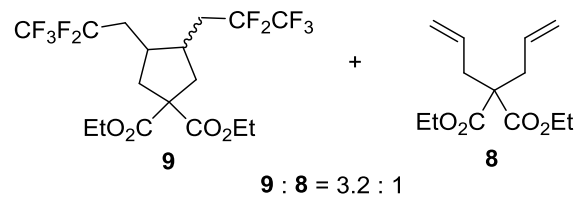

$\left({ }^{13} \mathrm{C}\right.$ NMR, $\left.\mathrm{CDCl}_{3}, 101 \mathrm{MHz}\right)$
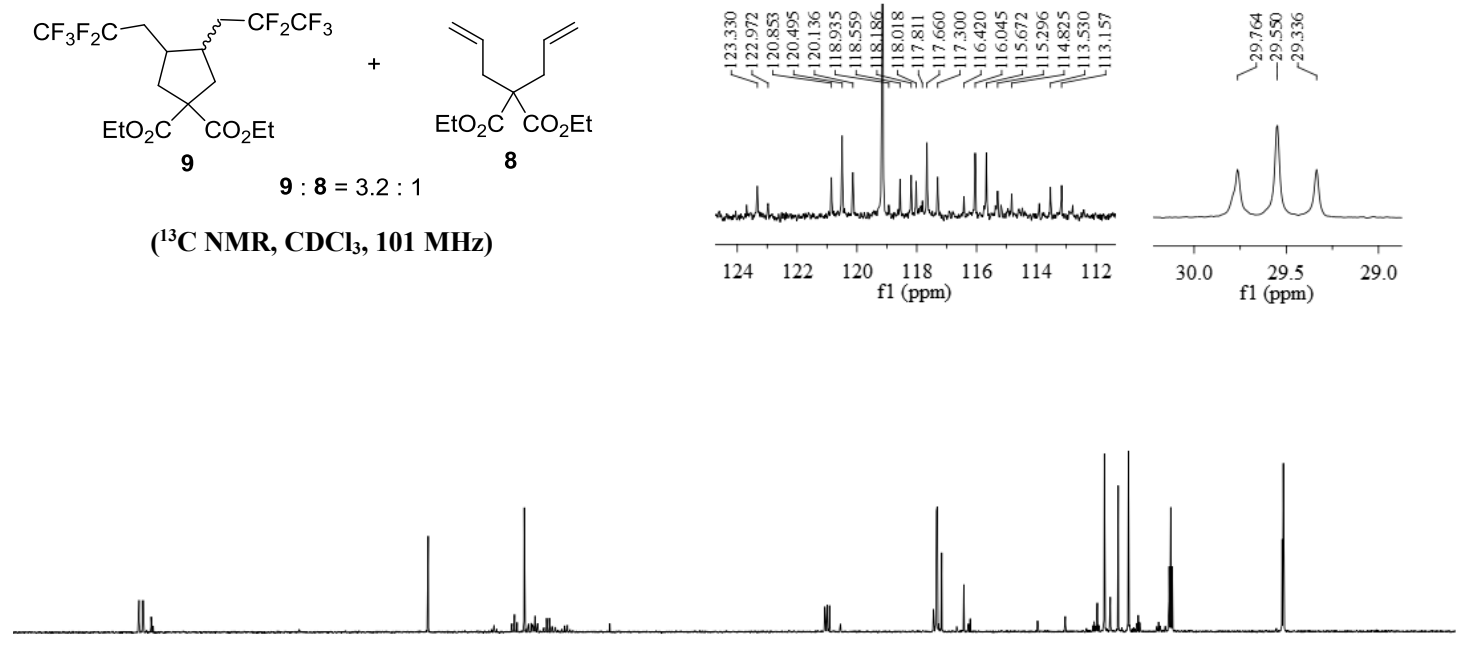

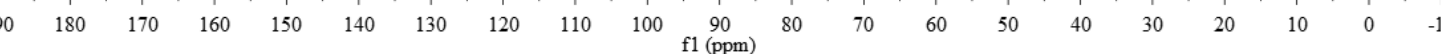

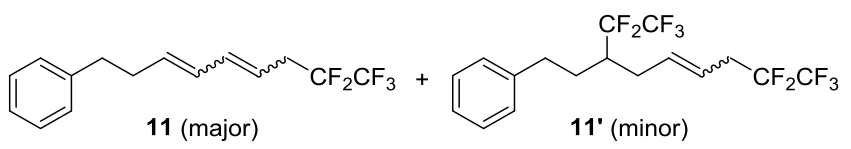

( ${ }^{1} \mathrm{H}$ NMR, $\left.\mathrm{CDCl}_{3}, 400 \mathrm{MHz}\right)$

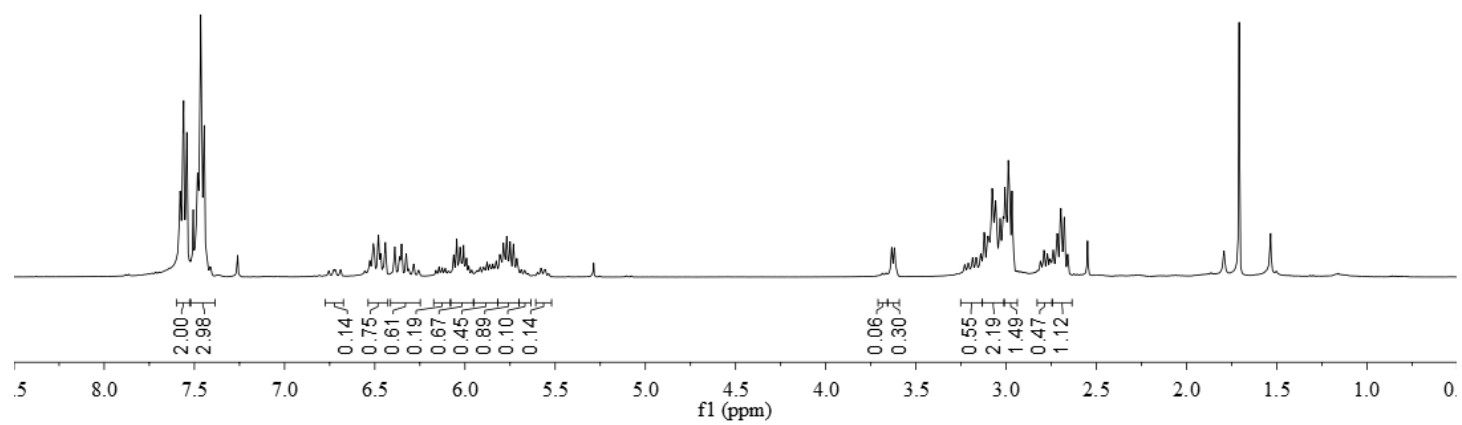




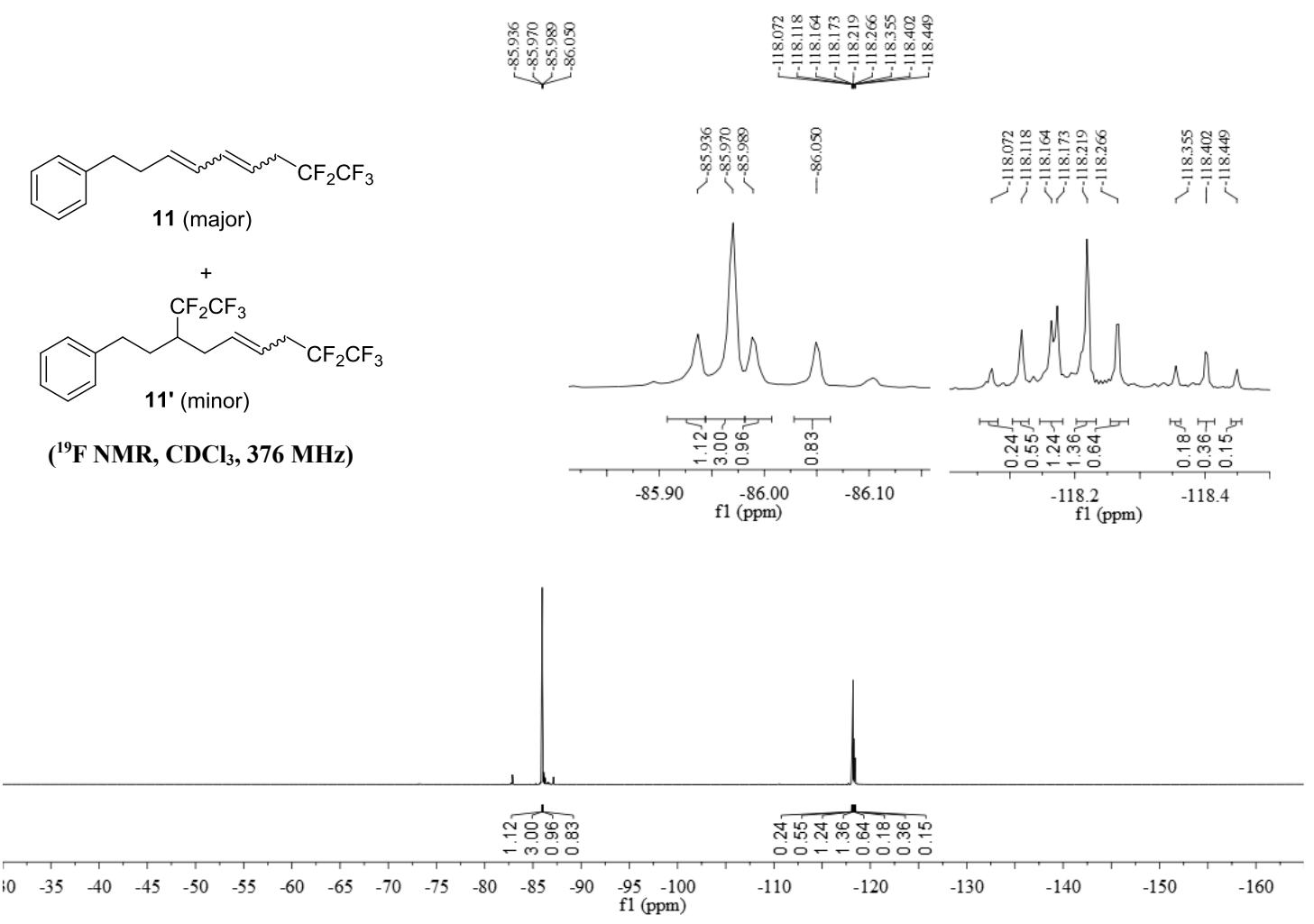

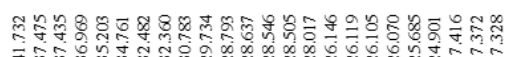

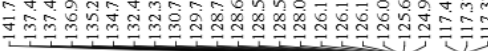

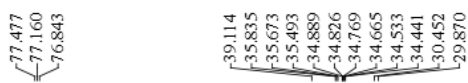

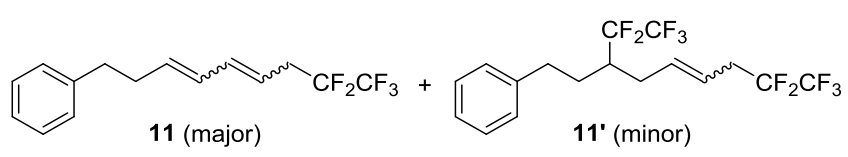

$\left({ }^{13} \mathrm{C} \mathrm{NMR}, \mathrm{CDCl}_{3}, 101 \mathrm{MHz}\right)$
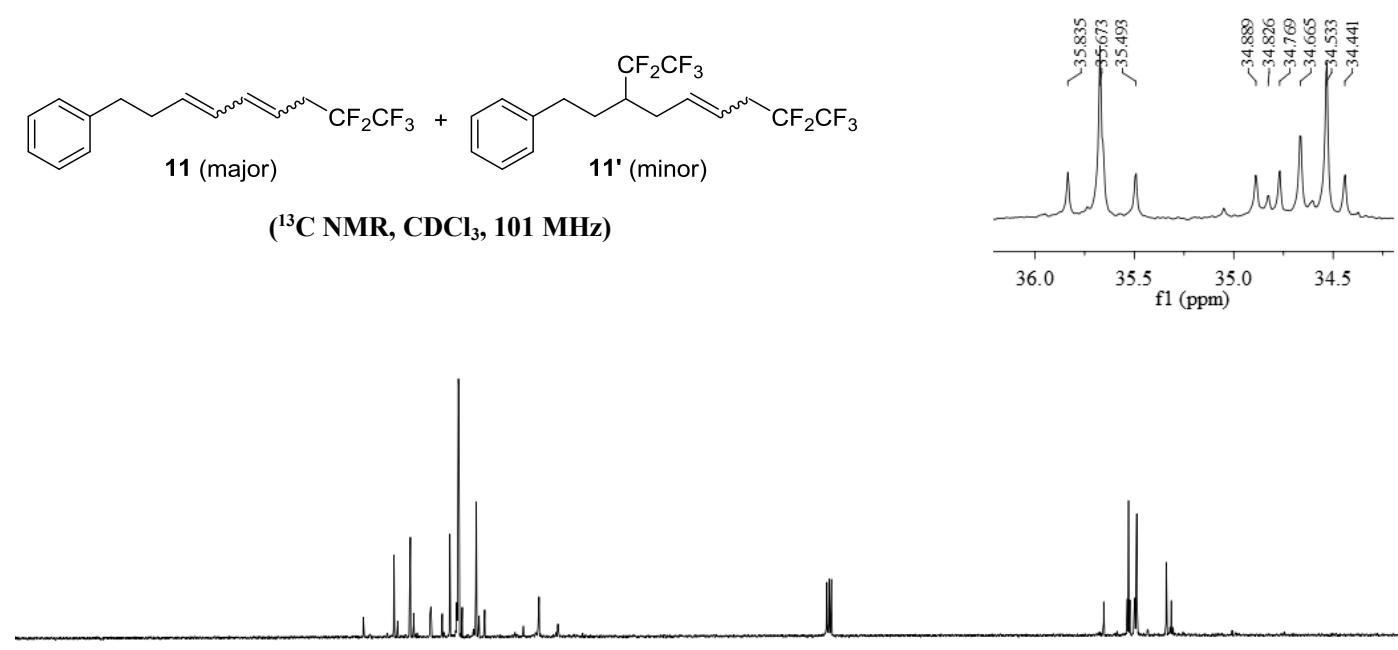

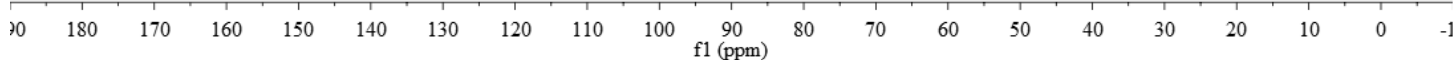




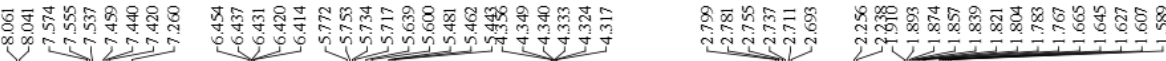

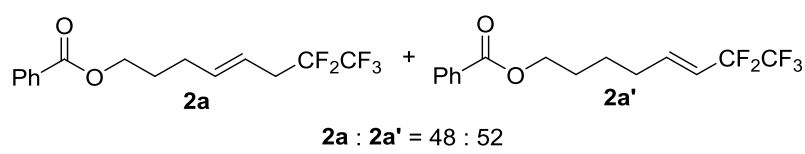

$\left({ }^{1} \mathrm{H}\right.$ NMR, $\left.\mathrm{CDCl}_{3}, 400 \mathrm{MHz}\right)$

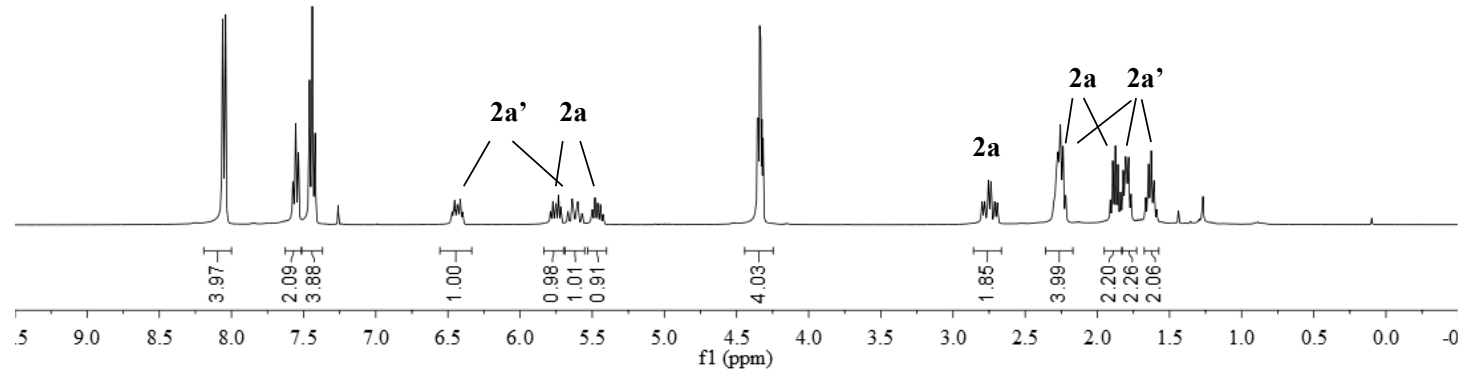

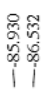

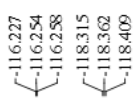

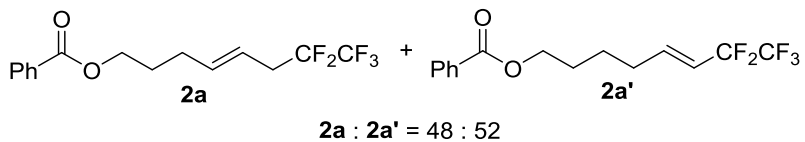

$\left({ }^{19} \mathrm{~F} \mathrm{NMR}, \mathrm{CDCl}_{3}, 376 \mathrm{MHz}\right)$
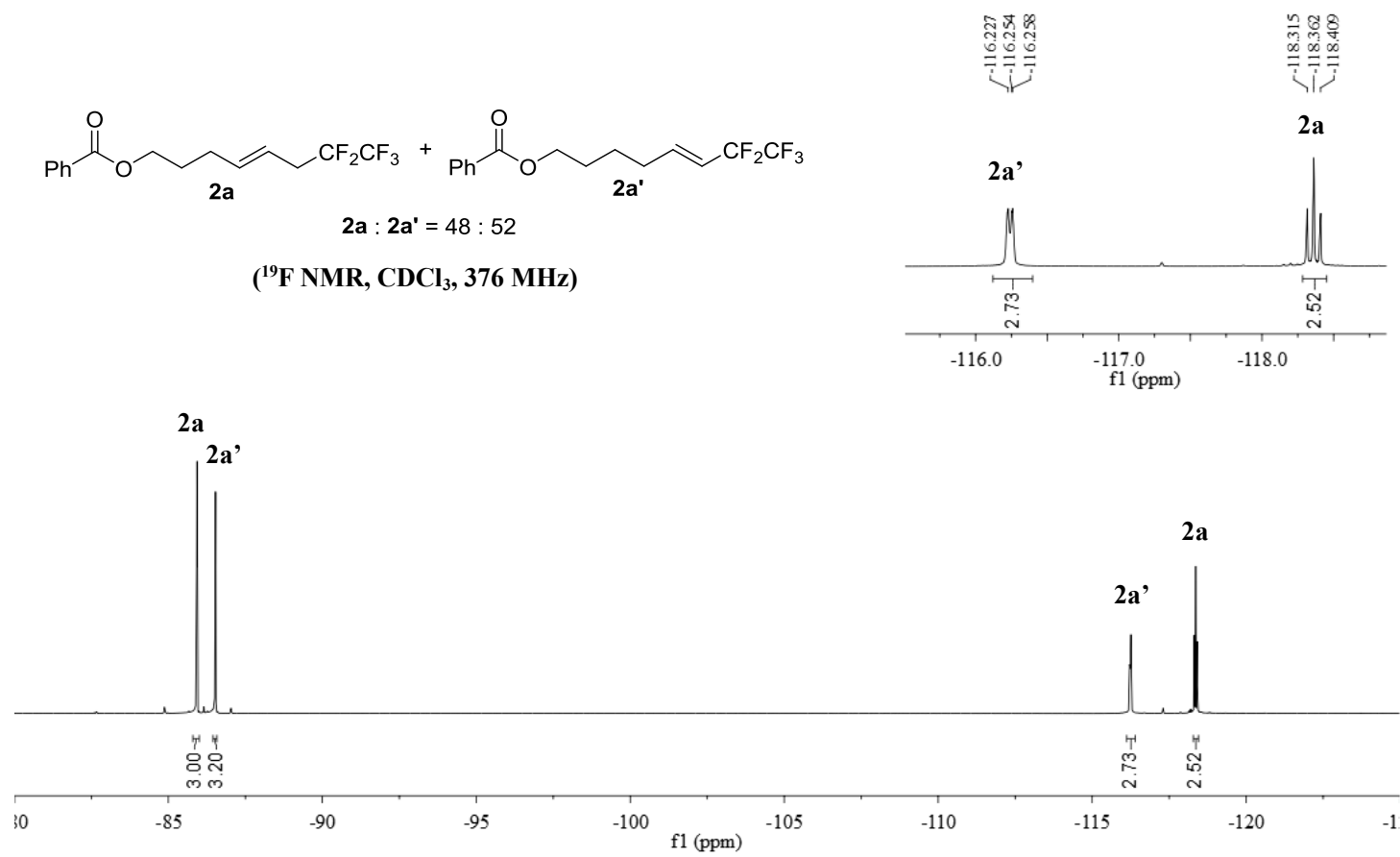


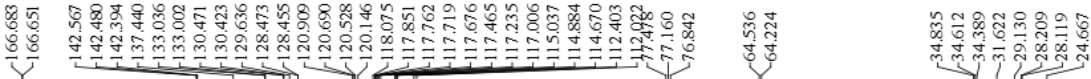

$\mathrm{CF}_{2 \mathbf{a}} \mathbf{2 a}^{\prime}=48: 52$

$\left({ }^{13} \mathrm{C}\right.$ NMR, $\left.\mathrm{CDCl}_{3}, 101 \mathrm{MHz}\right)$

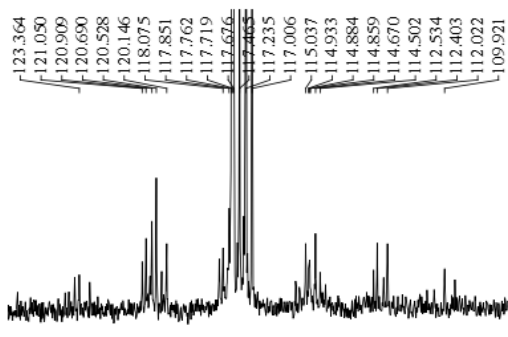

$\begin{array}{lllllllll}124 & 122 & 120 & 118 & 116 & 114 & 112 & 110 & 10\end{array}$

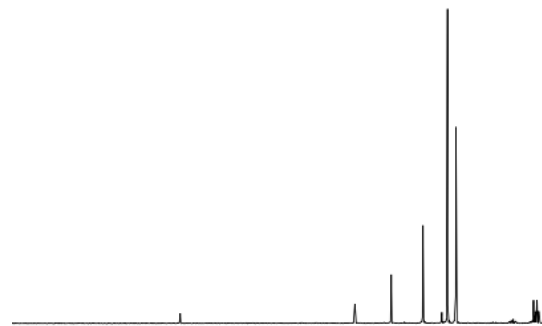

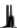

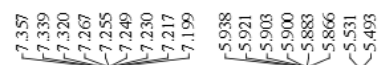

$\overbrace{\mathrm{D} \mathrm{D}}^{\mathrm{CF}_{2} \mathrm{CF}_{3}}$

$13\left({ }^{1} \mathrm{H}\right.$ NMR, $\left.\mathrm{CDCl}_{3}, 400 \mathrm{MHz}\right)$

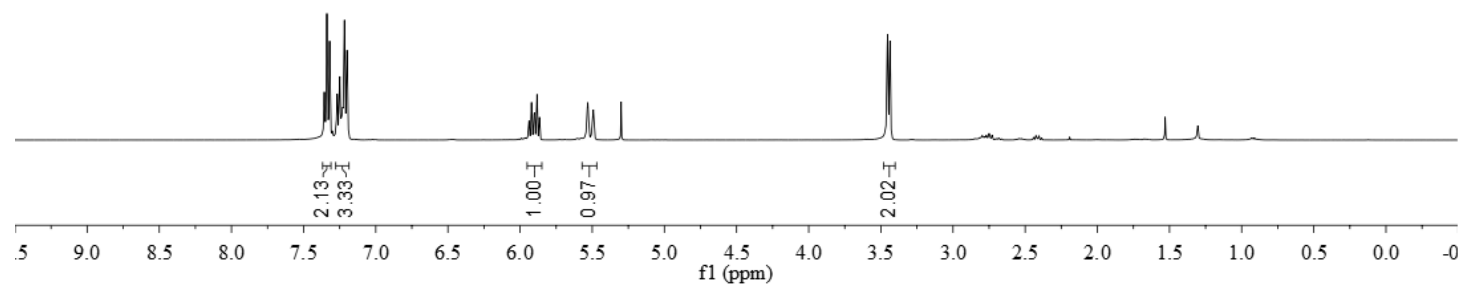




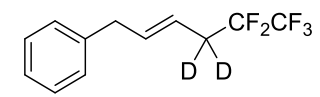

$13\left({ }^{19} \mathrm{~F}\right.$ NMR, $\left.\mathrm{CDCl}_{3}, 376 \mathrm{MHz}\right)$

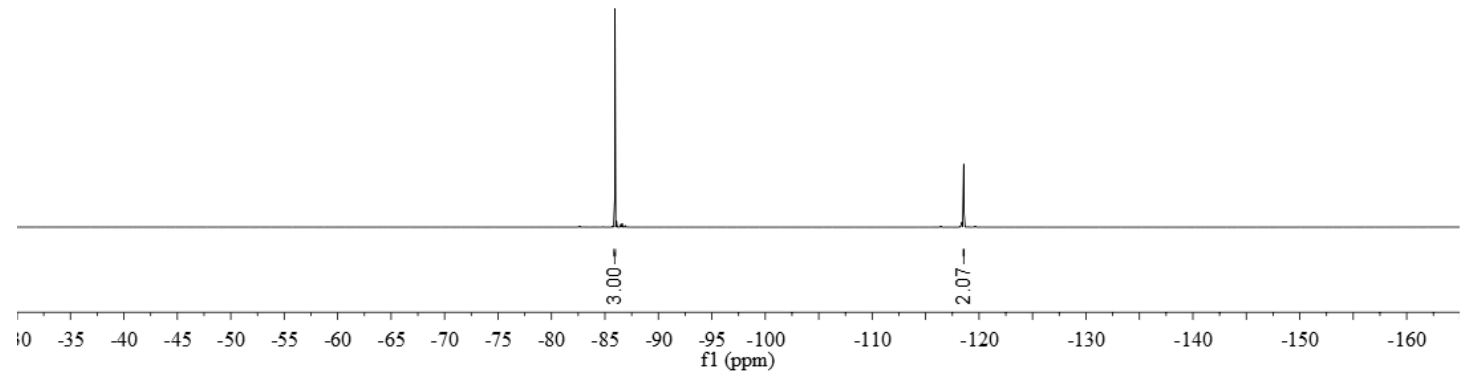

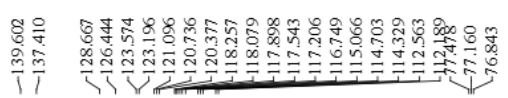

$\overbrace{D D}^{C_{D} C_{2} F_{3}}$

$13\left({ }^{13} \mathrm{C}\right.$ NMR, $\left.\mathrm{CDCl}_{3}, 101 \mathrm{MHz}\right)$
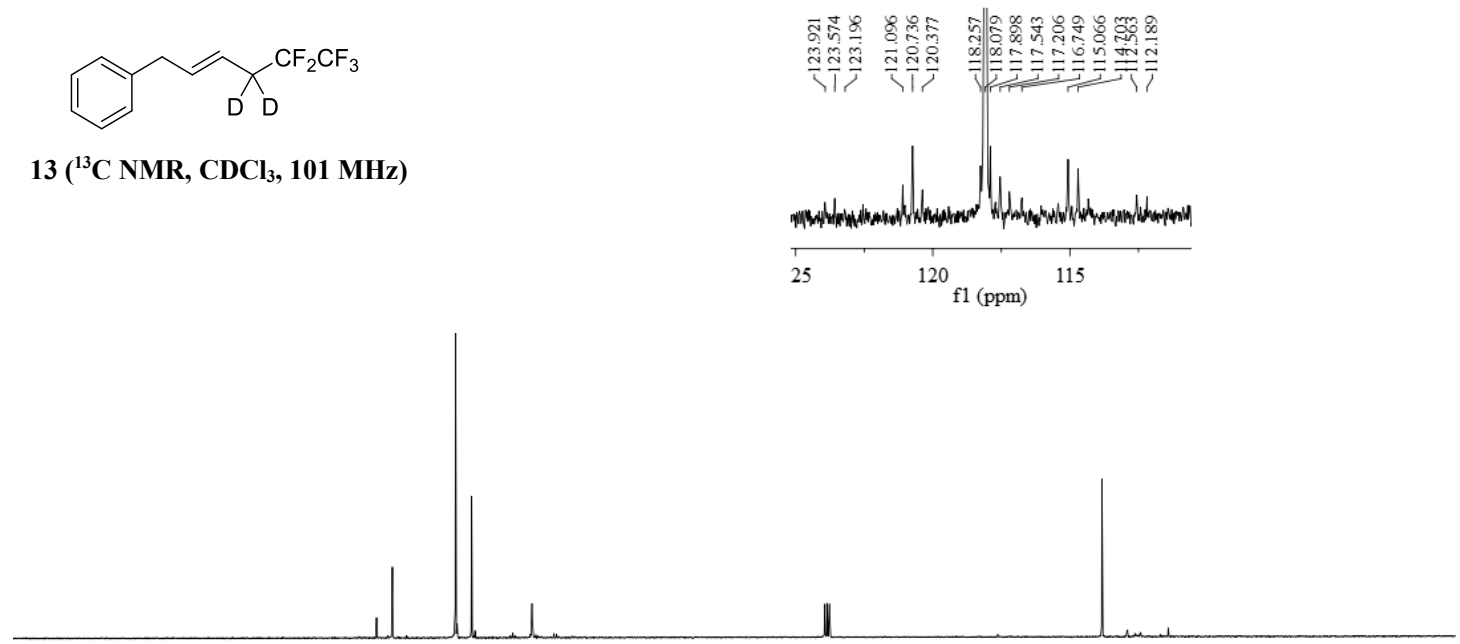

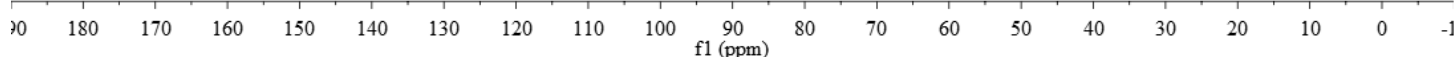

